\title{
A cross-cultural comparison of the role of some psychosocial factors in the etiology of coronary heart disease : follow-up to the Kaunas-Rotterdam Intervention Study (KRIS)
}

Citation for published version (APA):

Bosma, J. H. A. (1994). A cross-cultural comparison of the role of some psychosocial factors in the etiology of coronary heart disease : follow-up to the Kaunas-Rotterdam Intervention Study (KRIS). [Doctoral Thesis, Maastricht University]. Datawyse / Universitaire Pers Maastricht. https://doi.org/10.26481/dis.19940520jb

Document status and date:

Published: 01/01/1994

DOI:

10.26481/dis.19940520jb

Document Version:

Publisher's PDF, also known as Version of record

Please check the document version of this publication:

- A submitted manuscript is the version of the article upon submission and before peer-review. There can be important differences between the submitted version and the official published version of record. People interested in the research are advised to contact the author for the final version of the publication, or visit the DOI to the publisher's website.

- The final author version and the galley proof are versions of the publication after peer review.

- The final published version features the final layout of the paper including the volume, issue and page numbers.

Link to publication

\footnotetext{
General rights rights.

- You may freely distribute the URL identifying the publication in the public portal. please follow below link for the End User Agreement:

www.umlib.nl/taverne-license

Take down policy

If you believe that this document breaches copyright please contact us at:

repository@maastrichtuniversity.nl

providing details and we will investigate your claim.
}

Copyright and moral rights for the publications made accessible in the public portal are retained by the authors and/or other copyright owners and it is a condition of accessing publications that users recognise and abide by the legal requirements associated with these

- Users may download and print one copy of any publication from the public portal for the purpose of private study or research.

- You may not further distribute the material or use it for any profit-making activity or commercial gain

If the publication is distributed under the terms of Article 25fa of the Dutch Copyright Act, indicated by the "Taverne" license above, 


\section{A CROSS-CULTURAL COMPARISON OF THE ROLE OF SOME PSYCHOSOCIAL FACTORS IN THE ETIOLOGY OF CORONARY HEART DISEASE}

FOLLOW-UP TO THE KAUNAS-ROTTERDAM INTERVENTION STUDY (KRIS) 


\section{CIP-DATA KONINKLIJKE BIBLIOTHEEK, DEN HAAG}

Bosma, Johannes Hubertus Andreas

A cross-cultural comparison of the role of some psychosocial factors in the etiology of coronary heart disease : follow-up to the Kaunas-Rotterdam Intervention Study (KRIS). - Maastricht : Universitaire Pers

Maastricht. - III.

Thesis Maastricht. - With ref. - With summary in Dutch. ISBN 9052781354

Subject headings: coronary heart diseases ; psychosocial risk factors / coronary heart diseases ; mortality 


\section{A CROSS-CULTURAL COMPARISON OF THE ROLE OF SOME PSYCHOSOCIAL FACTORS IN THE ETIOLOGY OF CORONARY HEART DISEASE}

FOLLOW-UP TO THE KAUNAS-ROTTERDAM INTERVENTION STUDY (KRIS)

\section{PROEFSCHRIFT}

ter verkrijging van de graad van doctor aan de Rijksuniversiteit Limburg te Maastricht, op gezag van de Rector Magnificus, Prof. Dr. H. Philipsen, volgens het besluit van het College van Dekanen, in het openbaar te verdedigen op vrijdag 20 mei 1994 om 16.00 uur

door

Johannes Hubertus Andreas Bosma

geboren op 22 november 1963 te Limbricht

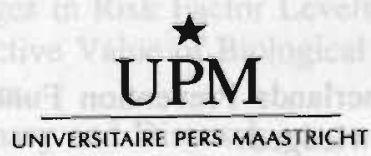


Promotores:

Prof. dr. A.P.W.M. Appels

Prof. dr. F. Sturmans

\section{Beoordelingscommissie:}

Prof. dr. J.W. van Ree (voorzitter)

Prof. dr. M.J. Drop

Prof. dr. P.G. Knipschild

Prof. dr. G.J. Kok

Prof. dr. J. Mackenbach (Erasmus Universiteit Rotterdam)

Financial support by the Netherlands Prevention Fund and the Netherlands Heart Foundation is gratefully acknowledged. 


\section{CONTENTS}

$\begin{array}{llll}\text { Chapter 1. Introduction } & 7\end{array}$

1.1 Aims of the Study 7

1.2 General Outline 10

Chapter 2. Study Populations in the KRIS Follow-up 11

2.1 Definition and Selection of Study Populations 11

$\begin{array}{lll}2.2 & \text { Recruitment and Participation } & 12\end{array}$

2.3 Differences between Participants and Nonparticipants 12

2.4 Follow-up: Data Collection 13

Chapter 3. Biological and Lifestyle Risk Factors:

Measurement and Distributions $\quad 17$

3.1 Biological Risk Factors 17

$\begin{array}{lll}3.2 & \text { Lifestyle Risk Factors } & 19\end{array}$

Chapter 4. Psychosocial Risk Indicators: Research Questions, Measurement, and Distributions 23

4.1 Socioeconomic Status 23

4.2 Social Network 27

4.3 Geographic Mobility 30

4.4 Type A Behavior Pattern 32

4.5 Stress and Exhaustion $\quad 35$

4.6 Self-rated Health 37

4.7 Family Aggregation of CVD 41

$\begin{array}{llll}\text { Chapter 5. Analyses } & 45\end{array}$

5.1 Strategy of Analysis $\quad 45$

$\begin{array}{lll}5.2 & \text { Statistical Methods } & 46\end{array}$

Chapter 6. Impact of Biological and Lifestyle Risk Factors 51

6.1 Age-adjusted Models 51

6.2 Multivariate Models 57

6.2.1 Effects of Risk Factors Simultaneously Adjusted 57

6.2.2 Smoking Habits 58

6.2.3 Effect Modification by CHD-at-screening 59

6.3 Changes in Risk Factor Levels 60

6.4 Predictive Value of Biological and Lifestyle Risk

6.5 Summary and Discussion 63 
Chapter 7. Impact of Psychosocial Risk Indicators

7.1 Socioeconomic Status 67

$\begin{array}{lll}7.2 & \text { Social Network } & 75\end{array}$

$\begin{array}{ll}7.3 \text { Geographic Mobility } & 79\end{array}$

$\begin{array}{lll}7.4 & \text { Type A Behavior Pattern } & 82\end{array}$

$\begin{array}{lll}7.5 & \text { Stress and Exhaustion } & 82\end{array}$

$\begin{array}{lll}7.6 & \text { Self-rated Health } & 85\end{array}$

7.7 Family Aggregation of CVD 92

7.8 Additional Predictive Value of Psychosocial Risk 96

7.9 Summary and Discussion 99

$\begin{array}{llll}\text { Chapter 8. Differences between Kaunas and Rotterdam } 111 & 11\end{array}$

8.1 Differences in Mortality and Nonfatal MI 111

8.2 Explaining the Differences 112

8.3 Summary and Discussion 114

$\begin{array}{llll}\text { Chapter 9. Summary } & 119\end{array}$

9.1 History and Aims of the KRIS Follow-up Study 119

9.2 Prevalence of Risk Factors and Indicators 120

9.3 Incidence of Main Endpoints 120

9.4 Impact of Risk Factors and Indicators 121

$\begin{array}{ll}\text { Samenvatting } & 127\end{array}$

$\begin{array}{ll}\text { References } & 131\end{array}$

$\begin{array}{ll}\text { Appendices } & 145\end{array}$

Dankwoord 163

$\begin{array}{ll}\text { Publications } & 165\end{array}$

$\begin{array}{lr}\text { Curriculum Vitae } & 167\end{array}$ 


\section{Chapter 1}

\section{INTRODUCTION}

\subsection{Aims of the Study}

A medically trained astronaut from a distant galaxy who embarked on a scientific expedition to the blue planet would have an exciting and disturbing trip. As he approached the earth, he would see a belt of coronary heart disease (CHD) somewhere between the 40th and 60th latitudes of the northern hemisphere. He would note an untouched island, which, he would later learn, is Japan. Coming closer to this belt, he would have the impression that the epidemic moves like a cloud or a wave from west to east. Coming even closer he would conclude that not all human beings are equally affected by this disease. When the wave starts to rise at a certain place, primarily the richest or best educated subjects seem to be affected. At its apex the wave afflicts all social classes equally and at its decline, especially the lower social strata. The astronaut might perceive a correlation between the shape of the wave and certain sociological and demographic characteristics. These include the number of new factories, the number of people moving from the countryside to towns, and an increased production of food.

Leaving his spaceship after landing and making a trip through the belt, he might note in his diary that CHD is called a managerial disease in some countries and a poor man's disease in others. He might also note that the disease seems to be less prevalent in societies where people often meet with many friends and more prevalent in societies where life is dominated by clocks and watches. Visiting some hospitals, he might note that people afflicted by the disease apparently have an aggressive and impatient lifestyle and some remarkable habits, such as inhaling the smoke of tobacco leaves.

Would the astronaut's observations have been correct? We do not know. Only careful measurements can give insight into the origins of the cardiovascular epidemic afflicting the industrialized countries. In an attempt to understand the origins of CHD and to obtain control over the major cause of death in the western world, many epidemiological studies have been done. Some of them seem to confirm the remarkable geographic distribution of the coronary epidemic. Swedish scientists, for example, have reported large regional variations in mortality risks due to CHD in mid-Sweden with a low prevalence in the east and a high prevalence in the west. Variations in risk factor levels do not explain this difference (Nerbrand et al., 1991). A World Health Organization work group collected the information from a total of 35 studies, most of them done in the 1970s, from 17 European countries. They covered a total population of 151,923 subjects. The results show significant geographic differences (Table 1.1). These differences cannot be explained by traditional somatic risk factors (Erica Research Group, 1991). 
Table 1.1

Estimated coronary mortality risk expressed as standardized mortality ratio in different European regions (men, age 40-59 years).

Northern Europe

Western Europe

Southern Europe

Eastern Europe
116.3

106.9

99.6

88.1

Figure 1.1 shows the dynamic pattern of the CHD epidemic. Although there were many differences in the initial CHD risks of the European countries, the eastern European countries showed a strong rise in the epidemic after 1970, whereas the western European countries had declining CHD risks.

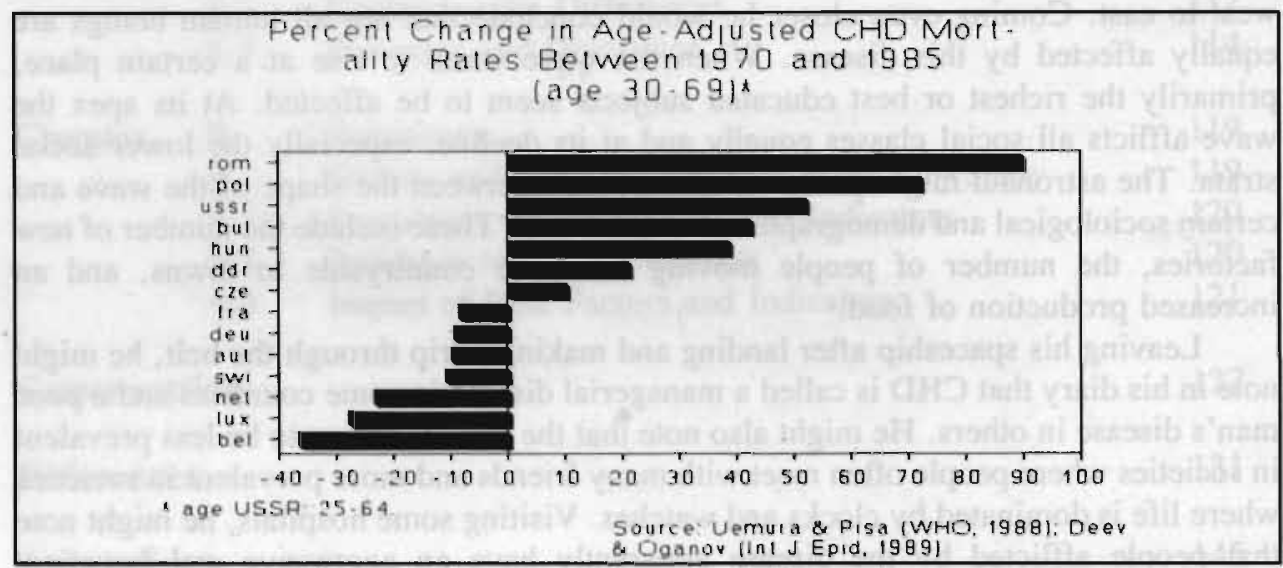

Figure 1.1 Percentage change in CHD mortality rates

The Kaunas-Rotterdam Intervention Study (KRIS) may provide some insight into the origins of the west-east gradient. A World Health Organization team from the Division of Research in Epidemiology and Communications Science initiated the study. It focused mainly on the problems of general methodology in intervention studies. The team chose the field of cardiovascular diseases (CVD) because they recognized that CVD are a major health problem in the industrialized countries of the world. Further, there was evidence for specific cardiovascular risk factors, and the pharmacological means of reducing these risk factors.

Before the World Health Organization formally approved the study, two national centers expressed considerable interest in participating. They are the Kaunas Medical Institute (Kaunas, a city of the former Lithuanian SSR) and the Rotterdam Municipal Health Department together with the Erasmus University (Rotterdam, The Netherlands). From the point of view of the future applicability of the results, it was considered particularly relevant that the study be conducted by these two centers, 
located as they were in two countries with different health service systems: a state supported one in the USSR and a predominantly private one in The Netherlands. Both centers wanted to participate mainly to develop a methodology for future large-scale CHD intervention studies.

Between 1970 and 1972 the study protocol describing the behavioral, epidemiological, and laboratory measurements, as well as the problems of data processing, was completed. The data collection phase extended from March 1972 to the end of 1974. In each city a sample of 4000 men between 45 and 59 years of age who represented various socioeconomic strata of an open urban population was identified. They were subjected to a baseline survey and subsequently invited to participate in the screening (Glasunov et al., 1981).

The original goal of a large-scale intervention trial proved impossible. Instead, the data were used for a cross-cultural follow-up study. This was feasible because of the continuous registration of all new cases of myocardial infarction (MI) and deaths in Kaunas, and because of an extended survey among the former Rotterdam participants in 1983. In 1988 both teams decided to cooperate in an analysis of the ten-year follow-up data. This cooperation was realized through the coordinating support of the World Health Organization European office in Copenhagen.

The desirability of a common follow-up study originated in the unique properties of the KRIS: the unusual amount of psychological and sociological information collected and the opportunity to cross-validate the predictive power of certain supposedly behavioral risk factors. Furthermore, the two populations involved were living in different socioeconomic conditions, one facing an increase (Lithuania) and one facing a decrease (Rotterdam) in the incidence of CHD.

Therefore, the emphasis of the data analysis is on the behavioral factors and some fundamental epidemiological questions formulated by the imaginary astronaut, such as: Does the gradient that associates the level of education with the incidence of CVD change in a given period as the epidemic develops? Is the incidence of CHD associated with parameters indicative of industrialization or "modernization", such as geographic mobility? Which demographic, sociological, and psychological factors contributed to the differences in the incidence of $\mathrm{MI}$ in both populations? Does social cohesiveness have a protective power?

A prospective study of two cohorts with a follow-up time of approximately 10 years offers a unique database. Paradoxically, its strength is also its weakness. The assessment of the exposure variables reflects the state of the art at the time the protocol was designed. It is hard to realize that in 1972 the epidemiological literature did not yet distinguish between HDL cholesterol and LDL cholesterol. Well-validated instruments for assessing job stress or social support were not available. Some demographic factors regarding education and occupation were classified information in Kaunas in 1972. The prevalence of some exposure factors may have changed during follow-up due to major or minor societal changes. These disadvantages do not outweigh the advantages. For example, the fact that the incidence of cigarette smoking decreased during follow-up does not invalidate the association of smoking with future CHD observed in this study. There is no doubt that the KRIS has its limitations. 
Fortunately, most of its somatic and psychological assessments still meet present standards. The data therefore still contribute to the social and behavioral epidemiology of CHD.

\subsection{General Outline}

Chapter 2 describes the populations, the screening, and the follow-up examinations in the KRIS. It also describes sampling strategies and participation rates. Some light is shed on differences between participants and nonparticipants.

Chapter 3 addresses the procedures for measuring of biological and lifestyle risk factors of CHD. No rationale for their assessment is presented because all of them are considered classic risk factors nowadays. The distributions of these risk factors in both cities are described and compared to see in a subsequent chapter whether differences in mortality and CHD incidence between Kaunas and Rotterdam can be explained by classic risk factors.

Chapter 4 addresses the procedures for measuring of the psychosocial risk indicators (we use the term risk indicators instead of risk factors because some of them, e.g., socioeconomic status (SES), increase the risk of CHD through still unknown pathways). Each section starts with brief review of the literature. We formulate specific hypotheses; the statistical technique and the selection of possible confounders are based upon the hypothesis tested. Then the instruments and the distribution of scores in Kaunas and Rotterdam are described.

Chapter 5 focuses on the overall strategy of the analysis and statistical techniques used to investigate the associations of characteristics measured initially with the mortality and incidence risks during the ten-year follow-up. The analyses usually start by computing age-adjusted associations. Then final models that include all risk factors are presented.

In Chapter 6 the impact of biological and lifestyle risk factors on all-cause mortality, mortality due to CVD, mortality due to CHD, and on nonfatal MI are investigated. Special attention is given to the curvilinearity, effect modification, change in risk factor levels, and the predictive value of biological and lifestyle risk factors.

Chapter 7 presents the results regarding the impact of psychosocial factors on the endpoints already mentioned. Separate analyses are done for SES, social network, geographic mobility, type A behavior pattern, stress, self-rated health, and family history of CVD. This chapter also addresses the additional predictive value of psychosocial risk indicators.

Chapter 8 presents the results of the comparison of mortality and incidence risks between the Lithuanian and Dutch populations. This chapter also shows results on which factors may contribute to this difference. This may add to our understanding of the west-east gradient.

In Chapter 9 the main results are summarized and recommendations for the application of the findings in public health or in future investigations are formulated. 


\section{STUDY POPULATIONS IN THE KRIS FOLLOW-UP}

\subsection{Definition and Selection of Study Populations}

A stratified cluster sampling scheme was chosen to recruit the participants for the KRIS because an assessment of the percentage of participation for different socioeconomically defined groups was very important. This led to a careful selection of the districts or quarters used for the sampling. The object was to choose areas that were homogeneous within themselves and heterogeneous with respect to one another.

The study population, which was to consist of 4000 subjects, was defined as men whose permanent home was within the predefined boundaries of a selected geographic region inside the city, and who were born between 1913 and 1927. In other words, they were at least 45 years old in 1972 but not yet 60 .

In Kaunas, residence in a geographic district was defined as the presence of an individual's name on the most recent voting list (that of 1971) for that district at the time of selection. This list was also supplemented with data from rental agencies before the start of the program. Men within the required age range who moved into a district after the start of the program but before the initial screening procedure had been completed were regarded as part of the study population.

Three districts were selected based on the type of housing. The first district consisted mainly of small private houses. The second district was in the downtown area where old apartment houses predominated. In the third district there were new high-rise buildings only. Due to errors in the first list of selected candidates and other factors, such as migration, the actual Kaunas sample consisted of 3553 men.

In Rotterdam, residence was defined as the presence of the individual's name on the roster maintained by the municipal computer center for a voting district 1 month before the program was initiated. Men within the required age range who moved into a voting district after that time but before the initial screening procedure had been completed were also regarded as part of the study population.

For the selection of districts use was made of the City Planning Department's study of the social typology of the districts in 1960. In this study each district was given a score based on the occupation and education of the population. The districts were classified in four groups according to this score, and a representative district was chosen from each group. In each district a subdistrict was chosen at random and all men within the required age range were selected. Adjacent subdistricts were then surveyed one at a time until the required number of subjects had been obtained. Due to migration and other reasons, such as exclusion of double entries, the final sample contained 3882 subjects. Further details are given in Glasunov et al. (1981). 


\subsection{Recruitment and Participation}

All eligible subjects were prescreened to collect information concerning social and demographic characteristics, medical experiences, and personal attitudes towards health and the health services. The baseline questionnaire consisted of 61 questions, including two concepts tested by means of a semantic differential test.

Seventy-one percent of the sample population in Kaunas and $88 \%$ of the sample population in Rotterdam participated in the baseline survey. It revealed that 166 subjects in Kaunas and 29 subjects in Rotterdam could not be invited for the screening examinations because of illness, death, or having moved out of the city. In principle all eligible subjects were invited to the screening examinations, irrespective of their participation in the baseline survey.

The numbers of subjects who participated in the baseline survey and/or in the initial screening examination are listed in Table 2.1. This table presents the cohorts of the follow-up study that is described further in Section 2.4. The cohorts are formed by those who participated in the screening, i.e., 3365 men (87\%) in Rotterdam and 2452 men $(69 \%)$ in Kaunas. The table also shows that some analyses using baseline items may have different numbers.

Table 2.1

Participation in baseline survey and/or initial screening in Kaunas and in Rotterdam.

\begin{tabular}{lrrrrr}
\hline & & Kaunas & \multicolumn{2}{c}{ Rotterdam } \\
& $\mathrm{N}$ & $\%$ & \multicolumn{2}{c}{$\mathrm{N}$} & $\%$ \\
Baseline and screening & 1903 & 53.6 & 3163 & 81.5 \\
Baseline only & 605 & 17.0 & 237 & 6.1 \\
Screening only & 549 & 15.5 & 202 & 5.2 \\
Neither baseline nor screening & 330 & 9.3 & 251 & 6.5 \\
Missing & 166 & 4.7 & 29 & 0.7 \\
Total & 3553 & 100.0 & 3882 & 100.0 \\
\hline
\end{tabular}

\subsection{Differences between Participants and Nonparticipants}

Participants in the screening in Kaunas were not found to differ from nonparticipants regarding any of the baseline questions. A later study by Domarkienne et al. (1987), however, showed that in Kaunas qualified professionals and subjects having a university education had the highest participation rates. Furthermore, in Kaunas allcause mortality within a follow-up period of 4.5 years was 2.5 times higher among the nonparticipants than among the participants.

The results of the baseline questions did not affect participation in the screening in Rotterdam either. However, an analysis of the participation rate per district based on all subjects showed that the lowest participation in the baseline interview and in the 
initial screening was observed in the district having the lowest SES. Nonparticipants in Rotterdam showed an increased mortality risk; $18 \%$ of the nonparticipants and $10 \%$ of the participants died during the ten-year follow-up (Appels et al., 1990).

A substudy was done in both places to identify other characteristics that distinguished participants of the screening from nonparticipants. In Kaunas the substudy focussed mainly on differences in health between participants and nonparticipants. Two samples of 100 subjects each were chosen at random from the group of participants and nonparticipants. The interviewers did their utmost to persuade the nonparticipants to undergo a medical examination. In spite of considerable effort, only 74 nonparticipants were willing to undergo the medical examination, so additional information on health status had to be obtained from available medical documents (outpatient records, etc.). The same information was collected for the 100 participants. There was no significant difference between participants and nonparticipants in the mean level of risk factors or other measured physiological variables (except the pulse rate, which was higher for nonparticipants). The additional information collected from medical records showed that ten subjects who absolutely refused to undergo the medical examinations had a pathological condition (hypertension, hypercholesterolemia, impaired glucose tolerance, previous MI or stroke). Adding this information to that obtained by the medical examination led to the conclusion that pathological conditions occurred twice as often in the nonparticipant group as in the participant group ( $51 \%$ versus $25 \%$ ). It was further noticed that a remarkable proportion of the nonparticipants $(31 \%)$ had a negative attitude towards medical personnel and examinations.

The substudy of nonparticipants in Rotterdam was aimed at assessing the subjects' knowledge of heart disease and risk factors, the subjects' social involvement in nonmedical activities, and some psychological data. For this purpose 110 participants and 115 nonparticipants were interviewed. The response rate for the substudy was $82 \%$ for participants and $57 \%$ for nonparticipants. The main reasons for not participating were "no time" and "already under treatment". Participants believed more often that checkups are necessary even if one is not sick and that special tests are worthwhile. More nonparticipants believed that examinations just make a person worry and that most health problems solve themselves. Nonparticipants needed more time to reach the examination center. According to the scores on the internal-external control scale, nonparticipants were more likely to think that fate is responsible for their health rather than their own actions and efforts.

\subsection{Follow-up: Data Collection}

The KRIS was initially a feasibility study, which, in the event that the results were positive, could eventually lead to a large-scale intervention study of CHD and cerebral stroke. In the intervention program of the KRIS, a number of men were invited to participate in an intervention trial for hypertension, hypercholesterolemia, or impaired glucose tolerance. Because of borderline values of blood pressure, 
cholesterol level, or glucose tolerance, 373 Lithuanians and 590 Dutchmen participated in a one-year double-blind intervention trial in which they were randomly assigned to a drug or placebo group. This trial was extended by another 4 years in Rotterdam. Furthermore, part of the Lithuanian population was screened twice additionally. In a rescreening 5 years later (in the period 1977-1980) all the subjects were invited and 1353 of them $(55.2 \%)$ actually participated. Another 10 years later in 1987 all Lithuanian KRIS participants were again invited to participate in a screening.

Morbidity and mortality data were somewhat differently collected in Kaunas and in Rotterdam. In Kaunas the follow-up data were collected from a continual mortality and morbidity registration. The Dutch data were collected through a survey done in March 1982. To make the follow-up time comparable, March 1, 1982 was chosen as the end date of an approximately ten-year follow-up for both cohorts.

\section{Kaunas}

Participants of the screening examinations in Kaunas were monitored by means of a mortality and MI register that covered the whole population of Kaunas. The list of MIs from the register was checked against the KRIS list once a year; the mortality register, monthly. Appendix I presents the Kaunas diagnostic criteria for MI. Causeof-death registration was based on the International Classification of Diseases (ICD), eighth version (World Health Organization, 1967). Table 2.2 presents the follow-up groups of the Lithuanian cohort.

Table 2.2 The Kaunas follow-up groups (March 1, 1982).

\begin{tabular}{lrrrr}
\hline & N & $\%$ & N & $\%$ \\
Lost to follow-up & 122 & 5.0 & & \\
Alive without any events & 1967 & 80.2 & & \\
Deceased & 303 & 12.4 & & \\
due to: CVD & & & 113 & 4.6 \\
$\quad$ CHD & & & 68 & 2.8 \\
$\quad$ non-CVD & & & 177 & 7.2 \\
$\quad$ unknown causes & & & 13 & 0.5 \\
Nonfatal MI & 60 & 2.4 & & \\
$\quad$ without subsequent death & & & 24 & 1.0 \\
$\quad$ with subsequent death & 2452 & 100.0 & & \\
\hline
\end{tabular}

${ }^{2} \mathrm{CHD}$ is a subgroup of CVD.

Section 5.2 presents some additional information about the 122 men who had to be classified as lost to follow-up. During follow-up 303 men died, of whom 24 also 
had a preceding nonfatal MI. In all, 84 men had had a nonfatal MI. Of those who died, 113 died of CVD (ICD codes: $390-459$ ), among which 68 of CHD (ICD codes: 410-414). The other cardiovascular deaths were caused by other heart diseases and cerebrovascular disease.

\section{Rotterdam}

In Rotterdam follow-up data were collected by inspection of the death certificates and by a questionnaire sent in March 1982 to all participants who, according to the records of the municipality in which they lived, were still alive.

Between screening and March 1982, 350 men died. The cause-of-death data were obtained from the Central Bureau of Statistics. The ICD, eighth and ninth versions, was used to register the cause of death (World Health Organization, 1967, 1977.).

To find out whether surviving Rotterdam participants had experienced a nonfatal MI, cerebrovascular accident, or angina pectoris, they were sent a letter with 14 questions. The final form of the questionnaire had been decided after three pilot studies (Appendix II). One reminder was sent three weeks later. Meanwhile, the study was introduced by letter to general practitioners and physicians. If answers to the questionnaire contained the slightest indication that a cardiovascular event could have occurred during the follow-up period, a physician contacted the patient's general practitioner or cardiologist.

The questionnaire was returned by 2731 (91\%) of the 3015 men surviving in 1982. Additional information from their general practitioners was needed for 440 responders. We contacted 153 general practitioners (51 outside Rotterdam). As much information as possible was obtained about the cardiovascular status of each responder from cardiologists' discharge letters and general practitioners' records. The necessary information was obtained for all except five subjects.

A coding scheme was devised to assess the probability that a participant had experienced a cardiovascular event. This coding was based on the physician's information about anamnesis, ECGs, and enzymes at the time of the event. This information was usually obtained from the cardiologist's discharge letters, but sometimes from the general practitioner's records. Subjects with indications of MI were classified as possible, probable, or definite MI victims. The coding system is explained in detail in Appendix III. It was found that 157 men had experienced a possible, probable, or definite MI.

A subsample of 30 nonresponders was contacted in person to investigate whether the presence of heart disease was associated with nonresponse. In this subgroup, only one nonresponder had, indeed, suffered from CHD. He had mailed the questionnaire after the deadline, but just before the substudy of the nonresponders (Appels et al., $1988_{\mathrm{a}}$ ).

A second check of the likelihood that not replying was associated with the cardiovascular status was made by comparing the mean risk factors of those who did and those who did not respond. Smoking prevalence was higher among the 284 who 
did not reply to the morbidity questionnaire. No differences were observed in prevalent CHD, cholesterol level, blood pressure, or age. It is, thus, highly unlikely that the experience of a nonfatal MI influenced the willingness to answer the morbidity questionnaire. It appears that nonresponse has not affected the random selection of men with or without heart disease.

Based on an estimated annual incidence of 7 per 1000, approximately 240 cardiac events were expected, slightly less than the 251 cases (CHD deaths and probable and definite nonfatal MIs) actually observed. These figures make it unlikely that the methods used for case finding resulted in a major over or under reporting of the total number of events. In short, the target population does not seem to be misrepresented by the participating population. Table 2.3 shows the different follow-up subgroups of the Rotterdam cohort. During follow-up 350 men died of whom 161 because of CVD (ICD codes: $390-459$ ). Of those who died of CVD, 125 were CHD victims (ICD codes: 410-414). In all, during follow-up 157 men had a nonfatal MI, 54 men had angina pectoris, $21 \mathrm{men}$ had bypass surgery, and $37 \mathrm{men}$ had a stroke. Because there is little evidence that those classified as "possible MI victims" suffered a welldocumented MI, analysis of the nonfatal MIs in Rotterdam was restricted to the 126 probable or definite MIs.

Table 2.3 The Rotterdam follow-up groups (March 1, 1982).

Nonresponders of the morbidity questionnaire (surviving) Alive with no events

Deceased

due to: CVD

CHD

non-CVD

unknown causes

Nonfatal MI

possible

probable

definite

Angina pectoris (without nonfatal MI)

Bypass surgery (without nonfatal MI)

Stroke (without nonfatal MI or bypass)

Total

N

284

2462

350

$\quad 10.4$

N

$\%$

${ }^{2} \mathrm{CHD}$ is a subgroup of CVD. 


\section{Chapter 3}

\section{BIOLOGICAL AND LIFESTYLE RISK FACTORS: MEASUREMENT AND DISTRIBUTIONS}

Many characteristics were assessed during the baseline survey and at screening. In close cooperation with the World Health Organization and the Center for Disease Control in Atlanta, USA, considerable attention was paid to the standardization, validity, and reliability of measurement and laboratory procedures. Laboratory personnel were exchanged, and invitations were sent to temporary advisors. Wellknown methods and questionnaires were used. The accuracy of the translations into Lithuanian and Dutch was checked by reverse translations. ECGs were exchanged, and measurements were duplicated. These measures, combined with the development of a fully elaborated and detailed laboratory protocol, resulted in a successful interlaboratory standardization. Details of the standardization procedures are presented elsewhere (Glasunov et al., 1981). Table 3.1 presents the information collected. In this table family aggregation of CVD is placed in the category of psychosocial risk indicators. This does not mean that genetic factors in the etiology of CVD or CHD are denied. This factor is categorized with the psychosocial risk indicators because in Chapter 7 much attention is paid to the influence of environmental factors shared by family members on family clustering of CVD.

Table 3.1 CVD risk factors and indicators in the KRIS.

\begin{tabular}{lll}
\hline Biological risk factors & Lifestyle risk factors & Psychosocial risk indicators \\
Age & Smoking & SES \\
Presence of CHD & Alcohol consumption & Social network \\
Systolic blood pressure & Physical activity & Geographic mobility \\
Diastolic blood pressure & & Type A behavior \\
Plasma cholesterol & & Stress \\
Plasma glucose & & Self-rated health \\
Body mass index & & Family aggregation of CVD
\end{tabular}

\subsection{Biological Risk Factors}

\section{Age}

Figure 3.1. shows the distribution of age in both populations. The Rotterdam population was only slightly older than the Kaunas cohort (mean: 52.5 and 51.7 respectively; $[P=0.00$ (Student's $t$-test)]. 


\section{CHD-at-screening}

More detailed information about the measurement procedures for the evaluation of the ECGs and the assessment of angina pectoris are presented in Appendix IV. In order of assigned priority, CHD-at-screening was defined using the following diagnostic criteria:

1. Definite MI: Minnesota codes I 1-2 (Q waves)

2. Abnormal ECG: Minnesota codes I 3, IV 1-3, V 1-3 or VII 1

3. Angina pectoris according to the Rose Questionnaire.

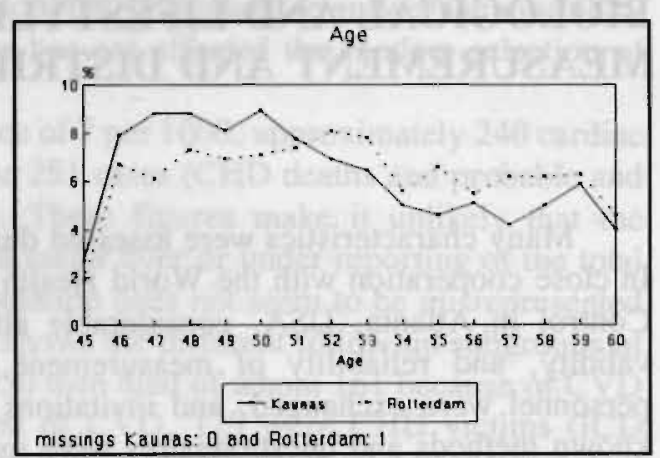

Figure 3.1 Age in Kaunas and in Rotterdam.

Table 3.2 shows the prevalence of CHD-at-screening. The generally greater CHD prevalence in Kaunas must be interpreted cautiously. It was probably due to technically imperfect ECGs, and while Kaunas investigators tended to code them as "pathological", Rotterdam coders did the opposite (Glasunov et al., 1981). Differences between interviewers also caused some differences in the measurement of angina pectoris. In Kaunas physicians did the interviewing, whereas nurses, who followed the Rose Questionnaire strictly, did the interviewing in Rotterdam.

Table 3.2 CHD prevalence in Kaunas and in Rotterdam.

\begin{tabular}{lrrrr}
\hline & N & $\begin{array}{r}\text { Kaunas } \\
\%\end{array}$ & N & Rotterdam \\
Definite MI & 84 & 3.4 & 75 & $\%$ \\
Abnormal ECG & 121 & 4.9 & 234 & 2.2 \\
Angina pectoris & 201 & 8.2 & 147 & 7.0 \\
No CHD & 2046 & 83.4 & 2909 & 4.4 \\
\hline
\end{tabular}

$P=0.00\left(\right.$ chi $^{2}$ test $)$

\section{Blood Pressure, Cholesterol Level, Glucose Tolerance and Body Mass Index}

Figures 3.2 and 3.3 present the distributions of blood pressure, cholesterol level, glucose tolerance and body mass index (Appendix IV provides detailed information about the measurement procedures of these risk factors). No differences between Kaunas and Rotterdam were found in mean systolic blood pressure. In Kaunas the 
mean diastolic blood pressure and the body mass index were higher than in Rotterdam. The difference in diastolic blood pressure was $8 \mathrm{mmHg}$ ( 88.1 and 80.0 , respectively). The mean body mass index was 27.1 and 25.5 , respectively. Mean plasma cholesterol level was higher in Rotterdam (201.3 and 193.0, respectively) as was the plasma glucose tolerance level (165.1 and 145.9, respectively). Except the systolic blood pressure, all differences in mean values between populations were statistically significant according to Student's $t$-tests.

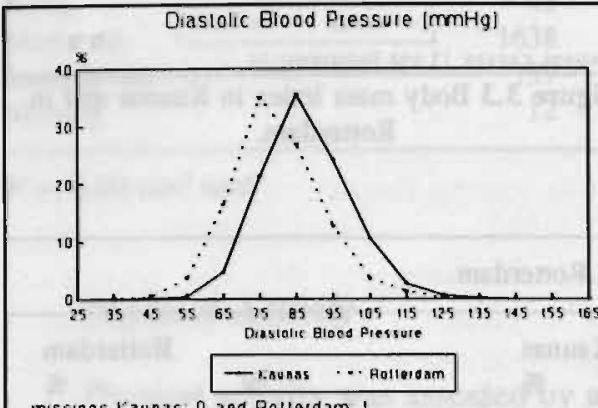

missings Kaunas: 0 and Rollerdam

Plasma Cholesterol (mg/100ml)

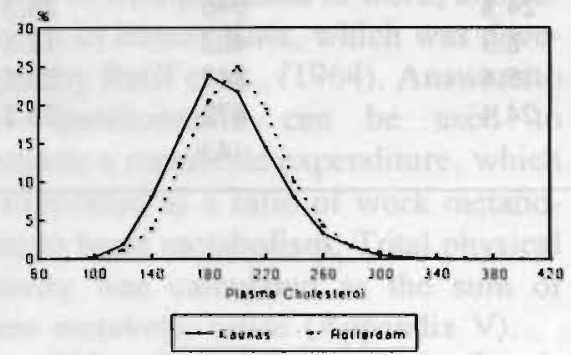

miscings Kaunas: 36 and Pollerdam 3
Systolic Blood Pressure $(\mathrm{mmHg})$

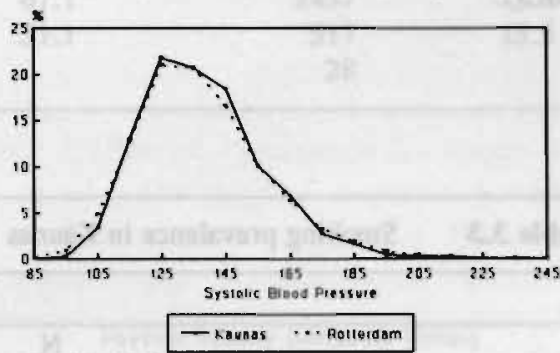

missings Kaunas: 0 and Rolleldam :

Plasma Glucose Tolerance (mg/100mi)

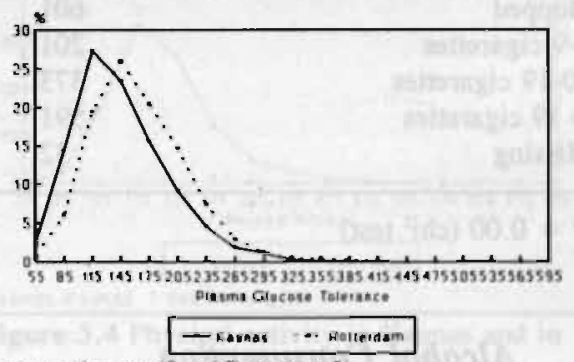

pmssings Kaunas 42 and Follerdam ?

Figure 3.2 Blood pressure, cholesterol and glucose tolerance in Kaunas and in Rotterdam.

\subsection{Lifestyle Risk Factors}

\section{Smoking}

Smoking habits were assessed by a questionnaire developed by Rose \& Blackburn (1968). Because only a small proportion of the subjects smoked a pipe or cigars (Kaunas $0.9 \%$; Rotterdam $4.3 \%$ ), they were treated as nonsmokers if they did not smoke cigarettes as well. Otten et al. (1988) reported no elevated risk for Rotterdammers smoking pipe or cigars; therefore, no relevant information is lost by coding pipe and cigar smokers as nonsmokers. 
Table 3.3 shows that smoking was more prevalent in Rotterdam. Ten percent more men smoked 10-19 cigarettes per day. Detailed analysis showed that $99.2 \%$ of Kaunas smokers tended to inhale cigarette smoke compared to $83.2 \%$ in Rotterdam. The mean age when smoking started was significantly lower in Rotterdam (16.8) than in Kaunas (20.3).

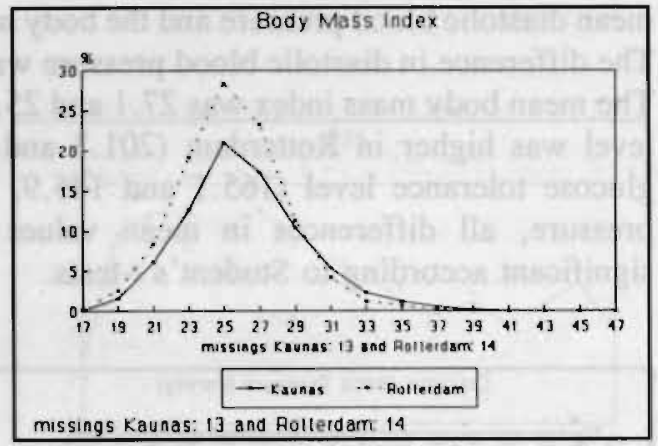

Figure 3.3 Body mass index in Kaunas and in Rotterdam.

Table 3.3 Smoking prevalence in Kaunas and in Rotterdam.

\begin{tabular}{lrrrr}
\hline & $\mathrm{N}$ & $\begin{array}{r}\text { Kaunas } \\
\text { \% }\end{array}$ & $\mathrm{N}$ & $\begin{array}{r}\text { Rotterdam } \\
\%\end{array}$ \\
Never & & & & 7.6 \\
Stopped & 654 & 27.0 & 254 & 28.5 \\
$1-9$ cigarettes & 601 & 24.8 & 946 & 12.1 \\
$10-19$ cigarettes & 201 & 8.3 & 403 & 25.5 \\
$>19$ cigarettes & 373 & 15.4 & 847 & 26.2 \\
Missing & 591 & 24.4 & 43 & \\
\hline
\end{tabular}

$P=0.00$ (chi ${ }^{2}$ test $)$

\section{Alcohol Consumption}

Alcohol consumption was assessed by a newly designed questionnaire, which is shown in Appendix V. Due to the small numbers in some categories of alcohol consumption, this variable was reduced to relatively crude categories: (1) no drinking; (2) moderate drinking: an unspecified amount of alcohol once a month at the most; or drinking up to 1 liter of beer, up to 0.5 liter of wine, or up to $100 \mathrm{cc}$ of spirits at least once a week; and (3) immoderate drinking: drinking more than 1 liter of beer or more than 0.5 liter of wine or more than $100 \mathrm{cc}$ of spirits at least once a week.

We do not know if those who drank more than one type of alcoholic beverage did so each time they drank. The number of men who drank moderate amounts of beer, wine, and spirits on the same occasion was assumed to be small and as such not leading to a severe underestimation of alcohol consumption.

Alcohol consumption was higher in Kaunas than in Rotterdam (Table 3.4). Drinking an immoderate amount of alcohol was twice as common in Kaunas. Further analysis showed that in The Netherlands beer and, to a smaller extent, spirits were the 
most preferred alcoholic beverages, whereas in Lithuania spirits were drunk by $97.2 \%$ of those who consumed alcoholic beverages.

Table 3.4 Alcohol consumption in Kaunas and in Rotterdam.

\begin{tabular}{lrrrr}
\hline & $\mathrm{N}$ & Kaunas & $\mathrm{N}$ & $\begin{array}{r}\text { Rotterdam } \\
\text { \% }\end{array}$ \\
None & & & & \\
Moderate & 43 & 1.8 & 383 & 11.5 \\
Immoderate & 1638 & 67.1 & 2437 & 73.0 \\
Missing & 759 & 31.1 & 517 & 15.5 \\
\hline
\end{tabular}

$P=0.00\left(\right.$ chi $^{2}$ test $)$

\section{Physical Activity}

Physical activity was assessed by a questionnaire on activities at work, means of transportation to work, and activities in leisure time, which was developed by Reiff et al., (1964). Answers to this questionnaire can be used to compute a metabolic expenditure, which is expressed as a ratio of work metabolism to basal metabolism. Total physical activity was calculated as the sum of these metabolic ratios (Appendix V).

Although Reiff's method of esti-

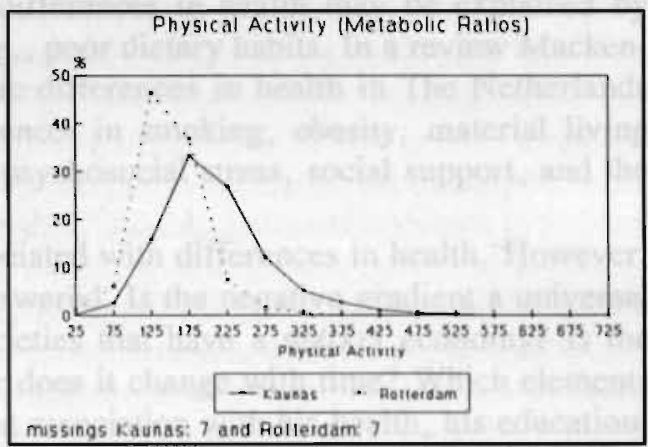

Figure 3.4 Physical activity in Kaunas and in Rotterdam. mating metabolic expenditure also included uniform metabolic ratios for the unspecified hours, these ratios were not applied, since variations among individuals might be too large for uniform ratios. Figure 3.4 shows that Kaunas men were much more often physically active than their Rotterdam counterparts. The mean physical activity was 208.48 in Kaunas and 151.11 in Rotterdam $(P=0.00)$ (Student's $t$-test). The differences for leisure activities were the most pronounced. Kaunas men spent much more time walking or engaging in moderate or heavy activities. 


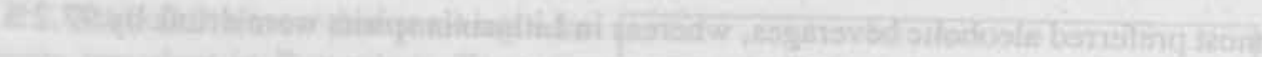

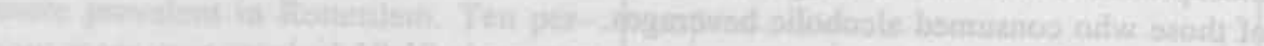

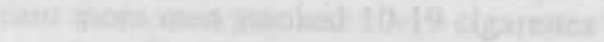

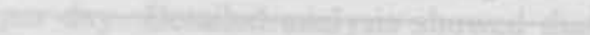

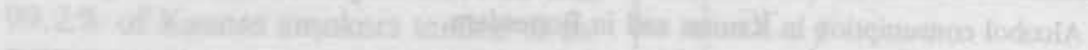

3. 5 witle

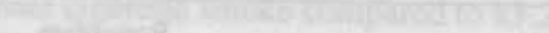

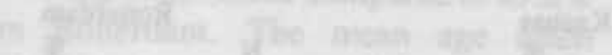

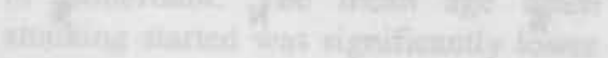

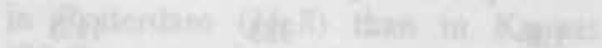

valoge

32

Tath

Bise

1. T.

15.5

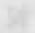

ant

(21)

whath

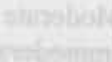

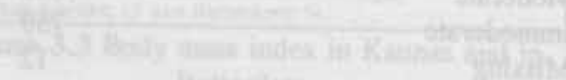

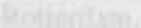

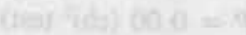

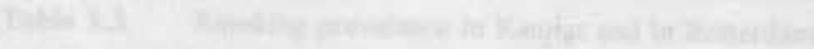

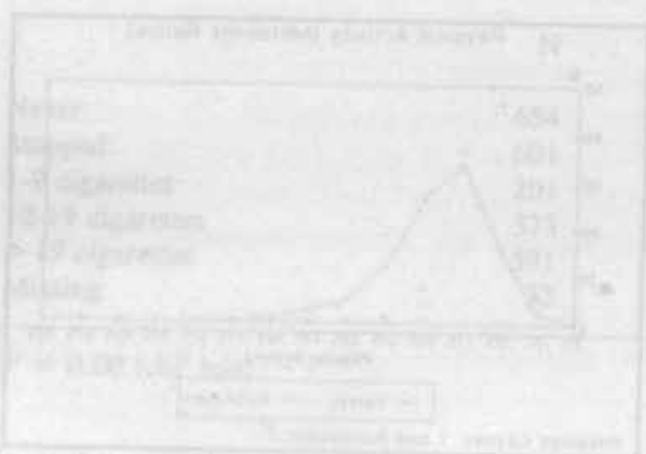

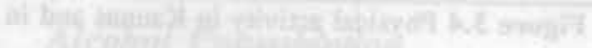

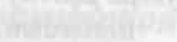

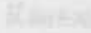

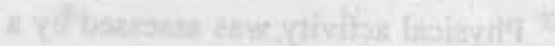

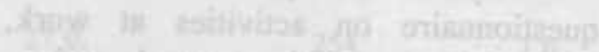

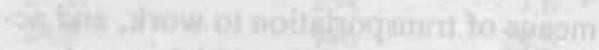

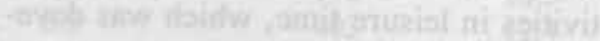

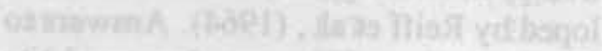

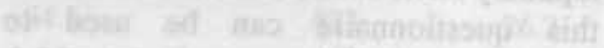

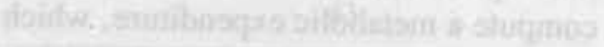

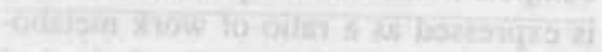

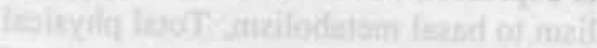

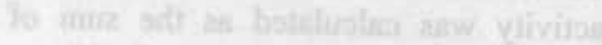

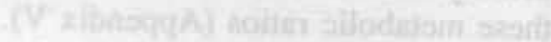

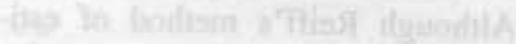

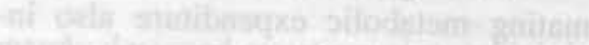

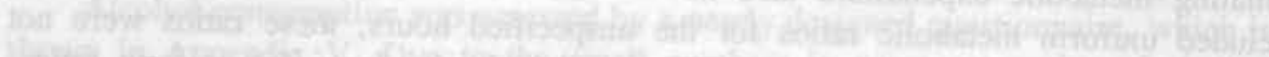

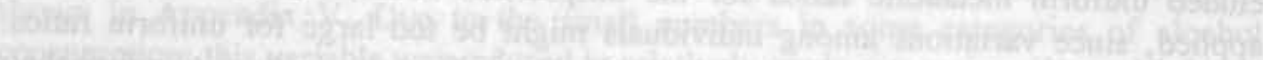

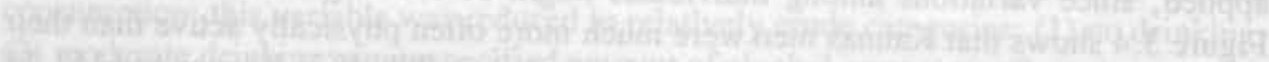

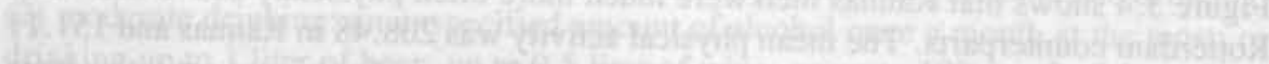

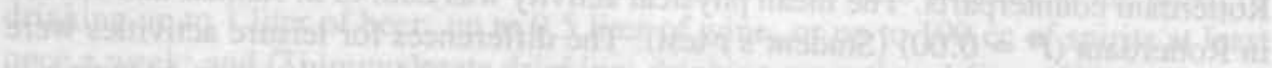

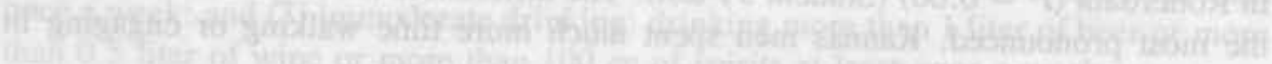

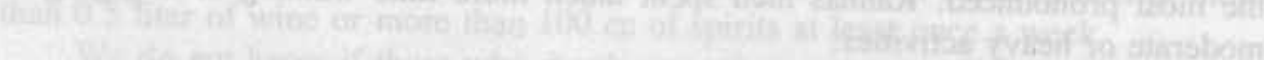

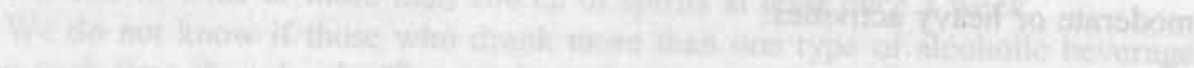

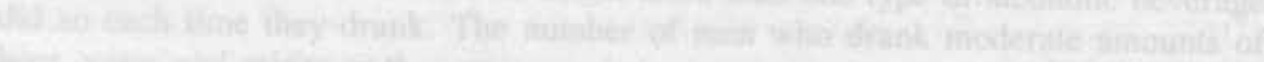

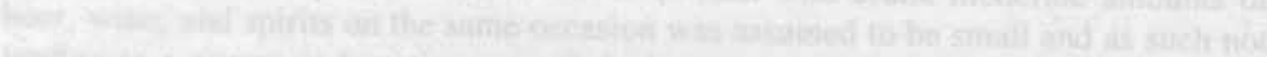

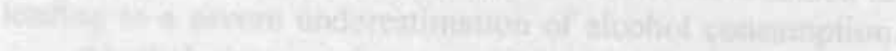

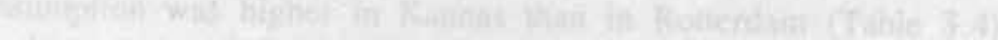
-

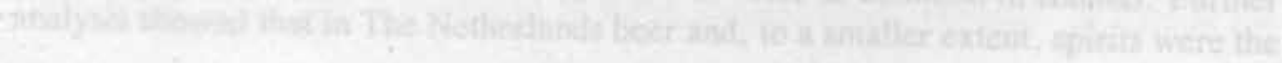




\section{PSYCHOSOCIAL RISK INDICATORS: RESEARCH QUESTIONS, MEASUREMENT, AND DISTRIBUTIONS}

\subsection{Socioeconomic Status}

\section{Research Questions}

Many studies give support to the belief that nowadays CHD is more prevalent among the underprivileged groups (Fox, 1989; Office of Population Censuses and Surveys, 1978; Slater et al., 1985; Welin et al., 1985). The negative gradient associating SES with practically all causes of death in western countries is, thus, well known. The similarity of this gradient across a wide range of diseases led Marmot et al. (1984) to argue that socioeconomic differences in health may be explained by factors affecting general susceptibility, e.g., poor dietary habits. In a review Mackenbach (1992) points out that socioeconomic differences in health in The Netherlands should be partly understood from differences in smoking, obesity, material living conditions, physical working conditions, psychosocial stress, social support, and the availability or use of health care services.

There is little doubt that SES is associated with differences in health. However, some fundamental questions remain unanswered. Is the negative gradient a universal phenomenon or does it exist only in societies that have a market economy? Is the negative gradient a stable phenomenon or does it change with time? Which elements defining a person's SES have the strongest association with his health, his education, his occupation, or the SES of his wife and family?

Concerning the first question, we note that the dominating ideology in former socialist countries considered inequities between social groups to be the product of a capitalist society. The goal of the new political system was to equalize the population socially. An essential feature of socialism was the eradication of inequalities in health between social groups differing in occupation or educational level. Health and social policies were designed accordingly. As a result, the former Soviet Union was the first to deliver health services free of charge and available to all.

Still, little information is available about the association of SES with health in the former socialist countries. According to Orosz (1990) in Hungary the incidence of death due to circulatory diseases in 1980 is lowest among skilled workers and highest among manual workers. Regional analyses in the former Soviet Union show that the highest incidence of CVD occurred in the most developed regions. Polish data also showed a positive relationship with development and living standards (Wnuk-Lipinski, 1990). Other studies confirm this picture of inequalities in health related to different social groups (Klinger, 1986; Mezentsva \& Rimachevskaya, 1992; Minev et al., 1990; 
Simon et al. 1986). However, due to lack of adequate statistics and contradictory results, it is unclear whether the gradient of the association of SES with CHD in eastern Europe is generally positive, negative, or absent.

As for the second question, there is some evidence for several western countries that the social class gradient did not always have a similar direction. Some studies suggest that the class pattern of deaths caused by CVD changed. These studies report that initially higher socioeconomic groups had the highest risk of dying of CHD, while later the reverse was true (Antonovsky, 1968; Kawachi et al., 1991; Kunst \& Mackenbach, 1990; Kunst et al., 1990; Mackenbach et al., 1989; Marmot et al., 1978; Morgenstern, 1980; Vagerö, 1991; Vagerö \& Norell, 1989). This phenomenon is usually explained by changing behavior patterns. Behavioral patterns predictive of CHD, such as increased intake of animal fat, smoking, and low physical activity, were first adopted by the well-to-do. Because of increased knowledge about the causes of diseases, this group was the first to change their lifestyle, causing CHD risks to decline in the higher SES groups. Hence, the original positive associations of SES with CHD mortality (higher status - higher CHD risks) may have reversed into an inverse association (higher status - lower CHD risks) (Morgenstern, 1980; Vagerö, 1991).

The third question addresses the components of a subject's SES, his education and his occupation. Special attention is given to the SES of a subject's wife because she has a central role in shaping the lifestyle of the family. Relatively little attention has been paid to the independent impact of the spousal SES on disease outcome. The number of studies addressing spouses' SES pales into insignificance beside the number of studies on background SES (e.g., father's occupational level) (Elford et al., 1991). This is rather striking, given the longer cohabitation with a partner than with a father. The wife's SES may have a better chance of affecting her husband's vulnerability to CHD. Some studies specifically investigate the effects of status inconsistency, i.e., the difference between the SES of spouses. These studies conclude that men whose wives had a higher SES than they themselves were at a higher risk of CHD compared to men whose wives had a similar or lower SES (Eyres, 1972; Shekelle et al., 1969; Suarez \& Barret-Connor, 1984).

The literature offers rather a mixed picture of the direct impact of the wife's SES on her husband's health and life expectancy. The Framingham study shows that men whose wives had 13 or more years of education were 2.6 times more likely to develop CHD than those whose wives had only an elementary school education (Haynes et al., 1983). Moreover, men whose wives were employed in white collar jobs were over three times more likely to develop heart disease than those married to blue collar workers or to housewives. Carmelli et al. (1985) and Eaker et al. (1983) found that the effect of a wife's educational level was only apparent for type A men. They concluded that a highly educated wife is deemed stressful by type A men only.

More recently Strogatz et al. (1988) found that men whose wives had more than 12 years of schooling had a relative risk of 0.80 for CHD compared to men whose wives had less education. Strogatz and colleagues explained this contrasting evidence in a partly younger and more recently investigated population by remarking that the 
meaning and impact of the educational level of wives are seemingly subject to change in the course of time.

The SES of the subject's parents is also examined for their association with his health, because differences in background SES are sometimes thought to contribute directly to socioeconomic differences in adult health. According to this hypothesis, socioeconomic differences in adult health originate during upbringing in families with different material conditions, as indicated by, for example, dietary habits, quality of housing and living conditions (Notkola et al., 1985; Nyström Peck, 1992; Östberg \& Vagerö, 1991; Power, 1991). Moreover, social and economic resources available to parents of higher social classes may also directly affect a person's well-being later in life. Therefore, we also investigate whether parents-in-law of higher social classes provide direct additional support through which men gain considerable benefit to their health.

Finally, since status inconsistency and intergenerational mobility are thought to result in conflicting role expectations that affect a person's self-image (Berting, 1965; Dressler, 1985; Shekelle et al., 1969; Syme et al., 1964; Vernon \& Buffler, 1988), these factors are investigated by testing the statistical significance of the interaction terms between (1) the participant's and his wife's SES, (2) the participant's and his parent's SES, and (3) the participant's educational and occupational levels. By doing so, it is possible to examine whether effects are heterogeneous or homogeneous.

\section{Assessment of SES}

The SES was investigated elaborately during screening. Information was obtained about the occupational and educational levels of the participants, and also about the SES of his wife, parents, and parents-in-law. Both educational levels and occupational levels were measured differently at both sites.

In Kaunas the subjects had to classify the number of years spent on elementary, secondary, special technical, university, and postgraduate education. The constructed "educational level" variable indicates which type of school was attended, thereby giving priority to the higher types of schooling. Participants who had no schooling at all $(\mathrm{N}=32)$ or who had postgraduate education $(\mathrm{N}=33)$ were added to the elementary school and university groups, respectively. Furthermore, the numbers of those who attended secondary education or special technical education were combined, resulting in the following categories:

1. elementary education

2 . intermediate level of education

- 3. higher education.

As it was classified information at that time, Kaunas investigators categorized the occupational level into rather crude groups ( 30 pensioners were excluded in the analysis of occupational level):

1. worker

2. employee. 
In Rotterdam the educational level was measured using the following categories: 1. primary education (lo) or continued primary education (vglo), lower general continued education (lavo) or some years of secondary education (ulo/mavo) only; 2 . similar to (1.), but with specific part-time education; 3 . diploma lower vocational education (lbo) or primary homemaking education (lhno) only; 4. similar to (3.), but with specific part-time education; 5 . diploma secondary education (ulo/mavo) or some years of preuniversity education (vhmo); 6 . similar to (5.), but with specific part-time education; 7. diploma preuniversity education (vhmo) or secondary vocational education (mbo); 8. diploma higher vocational education (hbo); 9. diploma university or academy. The educational levels were recoded as:

1. elementary education (values 1 and 2 from the original scale)

2 . intermediate level of education (values $3,4,5$, and 6)

3. higher (values 7,8 , and 9).

To measure occupational levels, Rotterdam investigators applied a sociological occupational scale that is frequently used in The Netherlands - the occupational register (beroepenklapper) of the Institute of Applied Sociology (Westerlaak et al., 1975). This scale has the following categories: 1 . unskilled laborer; 2 . skilled laborer; 3. lower-level employee; 4 . self-employed in small enterprise; 5 . intermediate-level employee (bookkeeper etc.); 6. higher occupation (not necessarily academic); 7. unemployed; 8 . other, specify... Since too little information was available about their SES, the unemployed $(\mathrm{N}=5)$ and those who did not fit into any of the categories 1 to $6(\mathrm{~N}=52)$ were considered missing. The occupational level was recoded as:

1. worker (values 1 and 2 from the original scale)

2. employee (values 3,4 , and 5 from the original scale)

3. higher occupation (value 6).

\section{Comparison between Kaunas and Rotterdam}

As Table 4.1 shows, most men in Rotterdam (47.3\%) and Kaunas $(56.8 \%)$ were workers. The Dutchmen appeared to have had only elementary education more often than the Lithuanians $(46.9 \%$ versus $30.0 \%$ ). However, the educational systems were not comparable and no information was available about the number of Lithuanians who actually acquired a specific educational certificate. Since the variables were not comparable, no attempt was made to test whether the differences were statistically significant. 
Table 4.1 Educational and occupational levels in Kaunas and in Rotterdam.

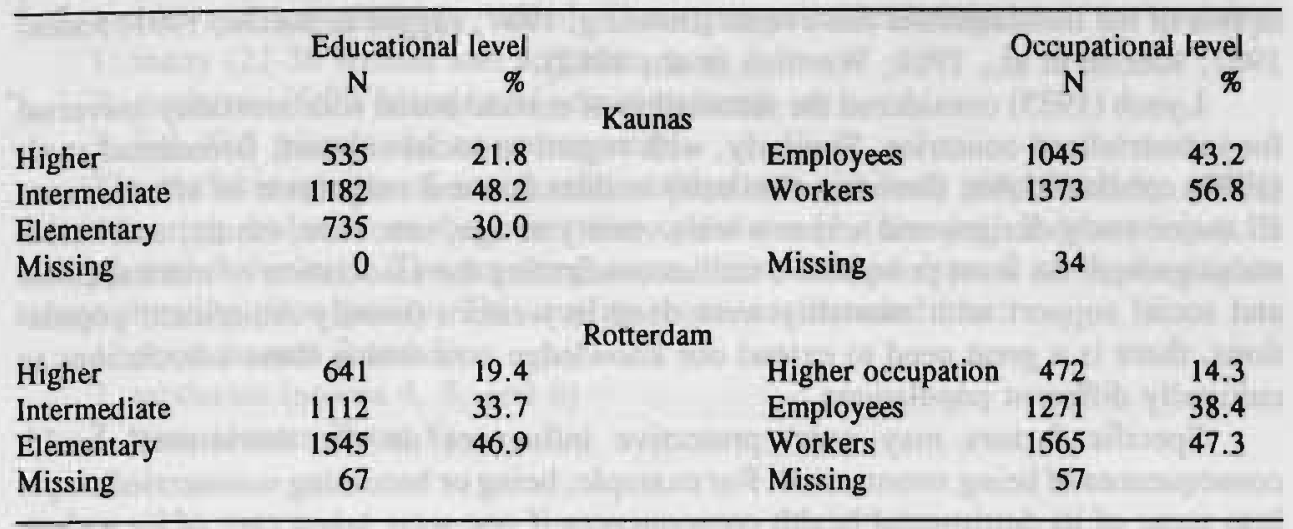

\subsection{Social Network}

\section{Research Questions}

During the last decades much attention has been given to the social support or the extent of the social network as a health protecting factor. The belief that the relative absence of social bonds makes people more vulnerable to mental and somatic diseases is supported by several studies. Several investigators found an increased likelihood of depression and other emotional disorders among those without an adequate social support network (Dean \& Ensel, 1982; Henderson, 1981; Paykel et al., 1980). Evidence of an increased risk of physical disorders and mortality to individuals without satisfactory social support is accumulating (Berkman \& Syme, 1979; Blazer, 1982; Broadhead et al., 1983; Hirdes \& Forbes, 1992; House et al., 1982, 1988; Kaplan et al., 1988; Orth-Gomer \& Johnson, 1987; Steinbach, 1992; Thomas et al., 1985; Vogt et al., 1992; Welin et al., 1992).

Marital status is most frequently incorporated in indices of social support. Separate studies of marital status show that the married have fewer health problems than the nonmarried. They have lower mortality risks, lower short and long-term hospital stays, and fewer visits to psychiatric hospitals (Gove, 1973; Goldberg \& Novack, 1992; Mendes de Leon et al., 1992; Ortmeyer, 1974; Rosengren et al., 1988). For example, Kraus \& Lilienfeld (1959) found age-adjusted relative risks of mortality of $1.47,1.46$, and 1.84 for single individuals, widows or widowers, and divorced individuals, respectively. Marital status is very important regarding all-cause mortality, death due to CVD, and cancer (Lynch, 1985).

Differences in mortality between the married and the nonmarried men are most frequently explained by the lack of social, emotional, and instrumental support experienced by the nonmarried men (Broadhead et al., 1983; House et al., 1988). For 
specific groups of nonmarried men other additional explanations have also been proposed. Divorce and bereavement are, then, seen as causes of emotional turmoil, as two of the most stressful life events (Bowling, 1987; Jagger \& Sutton, 1991; Jones, 1987; Kiecolt et al., 1988; Wertlieb et al., 1982).

Lynch (1985) considered the association of marital status with mortality universal for industrialized countries. Similarly, with regard to social support, Broadhead et al. (1983) concluded that there is a similarity in direction and magnitude of effect across all major study designs and across a wide variety of age, sex, race, ethnic, and health status groups. As most prospective studies confirming the association of marital status and social support with mortality were done in western (mostly American) populations, there is a great need to extend our knowledge concerning these associations to culturally different populations.

Specific factors may exert protective influences on the detrimental health consequences of being nonmarried. For example, being or becoming nonmarried might lose some of its detrimental health consequences if one were taken care of by a close network of friends and acquaintances with whom one could discuss problems and grievances. Silverstein \& Bengtson (1991) concluded that the excess mortality associated with the stress of being widowed can be partially offset by affectionate relations with adult children. These authors want to extend knowledge by asking investigators to examine protective influences of relationship other than those with children. Therefore, both the direct and the buffering effects of the extent and the quality of the social network of friends on mortality and morbidity are investigated. This buffering effect of social networks is also studied for other psychosocial factors in the KRIS.

\section{Assessment of Social Network}

No direct assessment of social support was made in the KRIS. During screening marital status was assessed, and in the baseline survey some questions were asked about a subject's social network: four questions about the number of friends and the quality of this friendship. The assessment of marital status included four categories:

1. married

2. single (never married)

3. widowed

4. divorced.

The questions regarding friendship were: Of the people you know (outside your family) how many would you say are your intimate friends? Of the people you know (outside your family) how many people would you say are your good acquaintances? Do you discuss serious personal problems with your friends or good acquaintances? If you had a serious personal problem, would your friends or close acquaintances make sacrifices to help you? The questions regarding friendship were combined into a variable "quantity" (first two questions) and "quality" (last two questions). 
Participants could respond to the first two questions (quantity) with the following answering codes: none, 1-3, 4-10 and more than ten. Both items were recoded as: 0 , 2,7 , and 15 and, subsequently, added. Three groups were distinguished for quantity:

1. many (21-30 friends and acquaintances)

2. moderate (6-20 friends and acquaintances)

3. few (0-5 friends and acquaintances).

The variable quality of social relationships was similarly pinpointed by adding the answering codes from the other two questions [1 (always), 2 (sometimes), 3 (rarely) and 4 (never)]. The resulting score, then, ranged from 2 to 8 , the higher scores showing poor quality. This quality score was recoded into:

1. good (scores 2 and 3 )

2. moderate (scores 4,5 , and 6 )

3. poor (scores 7 and 8 ).

\section{Comparison between Kaunas and Rotterdam}

The frequency distribution presented in Table 4.2 shows that more men in Kaunas were married $(93.5 \%$ versus $88.3 \%$ ). Being single or divorced was approximately twice as prevalent in the Dutch population $(5.0 \%$ and $9.9 \%$ in Kaunas and Rotterdam, respectively).

Table 4.2 Marital status in Kaunas and in Rotterdam.

\begin{tabular}{lrrrr}
\hline & N & $\begin{array}{r}\text { Kaunas } \\
\text { \% }\end{array}$ & N & $\begin{array}{r}\text { Rotterdam } \\
\%\end{array}$ \\
Married & 2292 & 93.5 & 2944 & 88.3 \\
Single & 57 & 2.3 & 191 & 5.7 \\
Widowed & 36 & 1.5 & 57 & 1.7 \\
Divorced & 66 & 2.7 & 141 & 4.2 \\
Missing & 1 & & 32 & \\
\hline
\end{tabular}

$P=0.00$ (chi ${ }^{2}$ test)

Table 4.3 and 4.4 show the distributions of the quantity and quality of the social network. Kaunas men appeared to have many more friends and acquaintances than Dutchmen. Whereas in Kaunas $93.4 \%$ of the men had more than five friends, only $72.9 \%$ had more than five friends in Rotterdam. The quality of the friendships also appeared to be better in Kaunas. According to our categorization, $22.6 \%$ of the Kaunasians and $16.5 \%$ of the Rotterdammers had a qualitatively good social network. 
Table 4.3 Quantity of social network in Kaunas and in Rotterdam.

\begin{tabular}{lrrrr}
\hline & $\mathrm{N}$ & $\begin{array}{r}\text { Kaunas } \\
\%\end{array}$ & $\mathrm{~N}$ & $\begin{array}{r}\text { Rotterdam } \\
\%\end{array}$ \\
Many & & & 523 & 18.7 \\
Moderate & 564 & 30.9 & 1511 & 54.2 \\
Few & 1141 & 62.5 & 756 & 27.1 \\
Missing & 121 & 6.6 & 575 & \\
\hline
\end{tabular}

$P=0.00\left(\right.$ chi $^{2}$ test $)$

Table 4.4 Quality of social network in Kaunas and in Rotterdam.

\begin{tabular}{lrrrr}
\hline & N & Kaunas & Rotterdam \\
& & $\%$ & & $\%$ \\
Good & 387 & 22.6 & 468 & 16.5 \\
Moderate & 1143 & 66.8 & 1772 & 62.6 \\
Poor & 180 & 10.5 & 590 & 20.8 \\
Missing & 742 & & 535 & \\
\hline
\end{tabular}

$P=0.00\left(\right.$ chi ${ }^{2}$ test $)$

\subsection{Geographic Mobility}

\section{Research Questions}

Geographic mobility is frequently investigated for its association with disease (He et al., 1991; Medalie et al., 1973; Reed et al., 1984; Salmond et al., 1989; Syme et al., 1964). Geographic mobility places an individual in a new sociocultural context where he is exposed to new behaviors, values, and expectations and where he is confronted with several adaptation processes. Two factors may be involved in this adaptation process. One is the incongruity between what a person has learned to be appropriate behavior and the behavior that is considered appropriate in the new environment. The other is the loss of social support from the old social network.

The lack of the expected reaction to appropriate behavior and the absence of social support is hypothesized to lead to a state of chronic arousal, resulting in increased risks of CHD. This increased risk of CHD for migrants can, furthermore, be hypothesized to decrease the longer the time one has had to adapt to the new environment.

Geographic mobility may be disruptive because the social network may be harmed, but it may also involve beneficial changes in SES. For example, men who grew up in rural areas may have moved to the city because economic prospects were 
better there. Not allowing for the occupational level could, then, result in underestimates of the actual effect of the stresses encountered during and after moving.

\section{Assessment of Geographic Mobility}

Kaunas and Rotterdam researchers used somewhat different questions and different categories to assess geographic mobility in the baseline survey. Based on the question: "During the first 20 years of your life, where did you live most of the time?", Kaunas researchers grouped the homes of the subjects in the following categories:

1. a town or city with more than 85,000 inhabitants in Lithuanian SSR; town or city with more than 100,000 inhabitants outside Lithuanian SSR

2. an industrialized town or city with 85,000 inhabitants or less in Lithuanian SSR; an industrialized town or city with 100,000 inhabitants or less outside Lithuanian SSR

3. a village (without industry) or the countryside.

According to a frequently applied coding scheme developed by the Dutch Central Bureau of Statistics, Rotterdam researchers used answers to the question: "What is the name of the municipality where you lived most of the time during the first 20 years of your life?" to group the homes of the subjects in the following categories (Central Bureau of Statistics, 1960): 1. a rural municipality with over $40 \%$ of the economically active male population in agriculture; 2 . a rural municipality with $20-40 \%$ economically active population in agriculture; 3 . an urbanized rural municipality with over $50 \%$ of the economically active male population working in the manufacturing industry and with the largest population cluster having less than 5000 inhabitants; 4 . an urbanized rural municipality with over $50 \%$ of the economically active male population working in the manufacturing industry and with the largest population cluster numbering 5,000 $-30,000$ inhabitants; 5 . a residential municipality with over $30 \%$ of the economically active male population commuting, and with over $60 \%$ of the total resident population aged 14 years or more as well as $60 \%$ of the commuters born elsewhere; and with over $60 \%$ of the wage-earners being salaried employees in the total of the economically active male population and the resident commuters; 6 . a country town with 2,000 10,000 inhabitants in the built-up area; 7. a small town with $10,000-30,000$ inhabitants in the built-up area; 8. a medium-sized town with $30,000-100,000$ inhabitants in the built-up area; 9. a large town with 100,000 or more inhabitants in built-up area. A separate category was formed by the men who were brought up abroad $(0)$. These detailed groups in Rotterdam were combined as follows to make this variable more comparable to the measurement applied in Kaunas:

1. large towns (value 9)

2. small towns (values 3-8)

3. villages (values 1 and 2 ). 
Additional information was also gained about the number of years of residence in Kaunas or in Rotterdam.

\section{Comparison between Kaunas and Rotterdam}

Table 4.5 shows that many more Rotterdammers grew up in a large town than Kaunasians did (83.9\% versus $51.9 \%$ ). The large town where most of the Dutchmen grew up was Rotterdam itself. In Kaunas only $1.1 \%$ of all the men had lived in Kaunas for more than $80 \%$ of their lives, whereas the corresponding percentage in Rotterdam was $67.2 \%$. Additional analysis revealed that $49.8 \%$ of all the Rotterdammers had lived in Rotterdam from birth onwards. In Kaunas only $0.2 \%$ had always lived in Kaunas. In Rotterdam 87 men had been brought up abroad.

Table 4.5 Urbanization level of childhood home (I) and percentage of life spent in Kaunas and in Rotterdam (II).

\begin{tabular}{|c|c|c|c|c|c|}
\hline 1 & N & $\%$ & II & cints & wore \\
\hline Large town & 944 & 51.9 & $80-100$ & 20 & 1.1 \\
\hline Small town & 408 & 22.4 & $60-79$ & 32 & 1.7 \\
\hline \multirow[t]{3}{*}{ Village } & 467 & 25.7 & $40-59$ & 244 & 12.8 \\
\hline & & & $20-39$ & 779 & 40.9 \\
\hline & & & $0-19$ & 828 & 43.5 \\
\hline Missing & 633 & & Missing & 549 & \\
\hline Large town & 2522 & 83.9 & $80-100$ & 2082 & 67.2 \\
\hline Small town & 315 & 10.5 & $60-79$ & 206 & 6.6 \\
\hline \multirow[t]{3}{*}{ Village } & 169 & 5.6 & $40-59$ & 304 & 9.8 \\
\hline & & & $20-39$ & 346 & 11.2 \\
\hline & nat & & $0-19$ & 162 & 5.2 \\
\hline Missing & 359 & & Missing & 265 & \\
\hline
\end{tabular}

\subsection{Type A Behavior Pattern}

\section{Research Questions}

The American cardiologists Friedman and Rosenman were the first to report an association of an overt behavioral disposition and the risk of CHD (Friedman \& Rosenman, 1959; Rosenman and Friedman, 1961). They described this "type A behavior" as: "...An action-emotion complex that can be observed in any person who 
is aggressively involved in a chronic, incessant struggle to achieve more and more in less and less time, and if required to do so, against the opposing efforts of other things or other persons, ... stemming from a fundamental and irretrievable sense of insecurity about the intrinsic value of the personality involved".

The finding of a positive association of this type A behavior with the incidence of future CHD in both the Western Collaborative Group Study and the Framingham Heart Study raised the question: Has this behavior pattern any predictive power in countries other than the United States? (Rosenman et al., 1975; Haynes et al., 1980). It is scientifically important to investigate whether this behavior pattern, which seemed to be characteristic of the achievement-oriented capitalist society, can also be observed in a socialist society.

It has been suggested previously that type $\mathrm{A}$ behavior may play a more decisive role in younger individuals (Falger, 1989). Although KRIS age ranges are very narrow (45-60), we also examine whether type A behavior is more strongly associated with CHD at younger ages. The interaction between subjectively experienced stress, as measured on the Reeder stress scale, and type A behavior is also addressed. Type A individuals who report large amounts of stress may form a group with extra highly elevated risks of future CHD.

\section{Assessment of Type A Behavior Pattern}

The type A behavior pattern was assessed during screening with form B of the Jenkins Activity Survey (JAS). Independent experts translated the questionnaire into Dutch and Lithuanian and back into English. Three of the questions were not included because they were considered typically American (e.g., "were you captain of an athletic team?"). Rewording was necessary in a few cases because of the need to express the question in terms compatible with the intent of the original sentence. Each reformulation was discussed with Jenkins. A pretest showed that the Dutch were less willing to compare themselves to other people. Therefore a midpoint category ("the same") had to be added to questions like "compared to others who do the same job as I do, my responsibility is....". For some questions there were not enough response alternatives. To the question "if you tell your wife or a friend that you will meet him or her somewhere at a definite time, how often do you arrive late?" the response alternative "I am absolutely never late" had to be added, because $85 \%$ of the pretest sample said that they were never late.

Several methods were used to compute JAS scores. A tape containing the raw JAS data was sent to Jenkins, who computed the scores on the total type A scale and the three subscales "job involvement", "hard-driving/competitive", and "speed and impatience". Because of doubts about the content validity of some items in the Dutch culture, Appels developed a Dutch adaptation of the JAS. He used the assessment of type A behavior with the structured interview as the criterion (Appels et al., 1987). For similar reasons the JAS was hard to administer in Kaunas. Therefore it was decided to measure the type $\mathrm{A}$ behavior pattern by using the short $\mathrm{N}$ version of the 
JAS, which consists of 13 items (Jenkins et al., 1979). Responses to these items were differently weighted and, subsequently, combined as one type A scale. Items and response weights are presented in Appendix VI. A continuous score and a combined score were constructed. The first reflects more type A behavior with increasing scores. Using the prescribed cutoff points $-5,0$, and 5 , the men were categorized as extreme type B, moderate type B, moderate type A, or extreme type A (Jenkins et al., 1979).

\section{Comparison between Kaunas and Rotterdam}

Figure 4.1 shows that type $A$ behavior was somewhat more prevalent in the Lithuanian population, although not significantly. Means on the type A scale are -0.71 for Kaunas and -1.21 for Rotterdam $[P=0.14$ (Student's $t$-test $]$. Table 4.6 shows that the extremes of the type A behavior were more prevalent in The Netherlands, whereas men who exhibited moderate type A or B behavior were more frequently found in Kaunas.

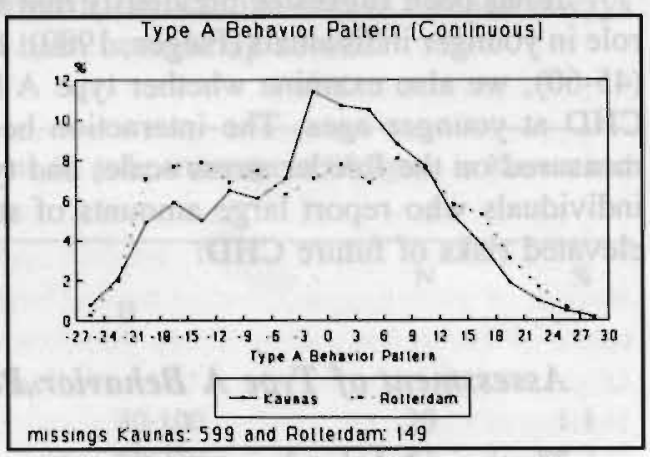

Figure 4.1 Type A behavior pattern in Kaunas and in Rotterdam

Table 4.6 Type A behavior pattern in Kaunas and in Rotterdam (combined score).

\begin{tabular}{lrrrr}
\hline & N & $\begin{array}{r}\text { Kaunas } \\
\%\end{array}$ & N & $\begin{array}{r}\text { Rotterdam } \\
\text { Extreme B }\end{array}$ \\
Moderate B & 613 & 33.1 & 1343 & 41.8 \\
Moderate A & 308 & 16.6 & 354 & 11.0 \\
Extreme A & 338 & 18.2 & 393 & 12.2 \\
Missing & 593 & 32.0 & 1126 & 35.0 \\
\hline
\end{tabular}

$P=0.00$ (chi $^{2}$ test) 


\subsection{Stress and Exhaustion}

\section{Research Questions}

The theory of "stress" is often used as the underlying explanation for various associations of social and psychological factors with the occurrence of disease. It is the most influential theoretical line of approach in behavioral medicine (Pearlin et al., 1981). However, there has been and there still is much controversy about what comprises "stress" (Feuerstein et al., 1986; Lolas \& Mayer, 1987). Is it a stimulus, a response, or an interaction between the two? A tentative definition is that "stress" describes "phenomena in which persons are placed at increased risk of disease by their exposure to or interaction with certain kinds of social occurrences or circumstances" (Dressler, 1985).

In modern behavioral medicine and social epidemiology stress is considered an "umbrella concept", and to have little meaning on its own. Modern scientists prefer to investigate the relation of specific behavior patterns, life events, or job characteristics to disease endpoints. However, at the start of the KRIS these ideas and the instruments to measure them had not yet been developed. The instrument that was used is outdated; it lacked clarity about the construct it measured. The seven items of the scale ask for rather different ideas, such as stable personality traits ("in general I am tense and nervous") or situational conditions ("I seldom experience tension in my family relations").

The scale also had one item asking for feelings of exhaustion. ("At the end of the day I am completely exhausted mentally and physically"). Many researchers have found excess fatigue to be a precursor of cardiac death in retrospective studies (Fraser, 1978; Kuller, 1978). This complaint is the most prevalent precursor of cardiac death. However, few prospective studies have been done to test the hypothesis that exhaustion is a risk indicator of cardiac death (Appels et al., 1988; Appels \& Otten, 1992). Therefore, we decided to look at the predictive power of exhaustion. We especially investigate whether feelings of exhaustion are a short-term risk indicator.

\section{Assessment of Stress and Exhaustion}

Stress was assessed during screening on the Reeder stress scale. This scale consists of five negatively and two positively formulated items, asking for several possible manifestations of "stress" (Reeder et al., 1968). The possible answers to the seven items measured during screening were: this describes me very well, fairly well, not very well, not at all. The items were:

1. In general I am tense and nervous.

2. I do not worry very much about my job.

3. My daily activities are very trying and stressful.

4. I seldom experience tension in my family relations.

5. There is a great amount of nervous strain connected with my daily activities. 
6. I often find tension in my relations with other people.

7. At the end of the day I am completely exhausted mentally and physically.

All of these items correlated positively in the Kaunas cohort. The two positively stated items ( 2 and 4 ) correlated positively with each other, but negatively with the other items in the Rotterdam cohort. The illogical pattern of correlations observed in Kaunas suggests a response set that casts serious doubts on the validity of the measurement of stress in Kaunas. Therefore, the analysis was restricted to the Dutch population.

Common factor modeling resulted in a one-factor solution for the Dutch population. Factor loadings are presented in Table 4.7. Although Cronbach's $\alpha$ is 0.76 , the percentage of variance accounted for by the factor is rather low (35.7\%). The stress factor score in Rotterdam was reduced to three categories (tertiles).

Table 4.7 Factor loadings based on the Reeder stress scale.

\begin{tabular}{lr}
\hline & Rotterdam \\
Item 1 & 0.58 \\
Item 2 & -0.62 \\
Item 3 & 0.70 \\
Item 4 & -0.35 \\
Item 5 & 0.72 \\
Item 6 & 0.56 \\
Item 7 & 0.58 \\
Percentage of & 35.7 \\
variance accounted for & \\
\hline
\end{tabular}

Item 7 was used as an indicator of exhaustion. The subjects were divided into three groups. The first group consisted of those who said that this statement (Item 7) did not describe them at all. The second group consisted of those who chose a midpoint category and the third group consisted of those who said that this item described them very well. The last group forms the index group because an extreme response is closest to the idea of exhaustion.

\section{Comparison between Kaunas and Rotterdam}

As no reliable data were available for Kaunas, no comparison could be made. The frequency distribution of exhaustion in The Netherlands is shown in Table 4.8. Of all the men $6.9 \%$ reported feelings of exhaustion. 
Table 4.8 Exhaustion in Rotterdam (Item 7).

\begin{tabular}{lrr}
\hline & N & $\%$ \\
Not exhausted & 1260 & 39.0 \\
Moderately exhausted & 1748 & 54.1 \\
Exhausted & 222 & 6.9 \\
Missing & 135 & \\
\hline
\end{tabular}

\subsection{Self-rated Health}

\section{Research Questions}

In recent years there has been a growing body of studies concerning the increased risk of mortality for people who evaluate their health as poor or bad. The response in these studies to the item: "Would you say your health is excellent, very good, good, fair, or poor?" appeared to reveal relevant information about longevity, even when baseline health status was accounted for (Idler \& Angel, 1990; Idler \& Kasl, 1991; Idler et al., 1990; Kaplan \& Camacho, 1983; Mossey \& Shapiro, 1982). The study by Mossey \& Shapiro (1982) reveals that self-rated health not only predicted threeyear mortality, but also six-year mortality. This subjective health assessment appeared to predict mortality even better than an assessment of the health status through the measurement of the number and the severity of illnesses reported by a physician or the individual himself.

An analysis of the data from a nine-year follow-up of the Human Population Laboratory cohort has shown that self-rated health has an independent association with cardiac and total mortality. Kaplan \& Camacho (1983) adjusted these data for an index of the physical health status, demographic factors, health behavior, and several psychological variables. They observe that people who perceived their health as poor had a 2.52 approximate relative risk of mortality compared to people who assessed their health as good (95\% confidence interval (CI): 2.36, 2.68). Eaker et al. (1992) make the following observation regarding a 20-year follow-up of 749 women who participated in the Framingham Study. Those homemakers who believed they were at increased risk of developing heart disease before there was any documented sign of CHD had an increased risk of MI.

In a representative American sample (NHANES I), Idler \& Angel show that selfrated health was associated with mortality over a 12-year follow-up period among middle-aged men ( $R R=2.8 ; 95 \% \mathrm{CI}: 1.5,5.3)$, but not among elderly men (older than 65 years) or women of any age. However, the study of Idler \& Kasl (1991) shows that self-evaluations were also significant predictors of mortality among men and women aged 65 years or more (Idler \& Kasl, 1991). 
The way in which the physical condition is assessed is crucial in determining the association of self-rated health with mortality. Self-reports about the physical condition might be affected by similar individual differences as the self-rating of health. This may result in an underestimation of the effect of self-rated health when the results of the self-reports are adjusted for. Adjusting for self-reported morbidity or recall of doctor-diagnosed diseases in studies from France and the United Kingdom may have resulted in a smaller and nonsignificant self-rated health/mortality association (Grand et al., 1990; Wannamethee \& Shaper, 1991). Only the study of Idler \& Angel (1990) did not rely heavily on self-reports. In this last study the physical health status was determined by medical diagnosis derived from a standardized medical examination.

The KRIS provides an opportunity to investigate longitudinally whether self-rated health is associated with mortality and morbidity when the results of a comprehensive and standardized physical examination are adjusted for. Further, the degree of crosscultural consistency of this association can be determined. The KRIS also may help find an answer to the question why self-rated health is associated with the mortality or morbidity risk. Most authors are mainly concerned about the possibility that the observed association, albeit consistent, is spurious. Eaker et al. (1992) suggest that the women of the Framingham study who perceived their health as poor may have based their expectation on their knowledge of family history of the disease. Others suggested that the rating of one's health as poor may reflect a still subclinical disease.

Modern social psychology offers an alternative or additional explanation. On giving an assessment of one's condition, one implicitly makes a social comparison. Social comparison is a cognitive process aimed at the maintenance of a positive selfimage. In stressful situations such as disease most people are inclined to compare themselves to others who are supposed to live under even more adverse conditions. Generally speaking, most people believe that they are better off than most other people. People believe that they are on the average more honest (Liebrand et al. 1986), better drivers (Svenson, 1981), have more positive and less negative personality traits (Brown, 1986), and a happier marriage (Buunk \& van Yperen, 1991). According to social psychologists, this "illusory superiority" serves to construct or preserve a positive self-image. This theory suggests that those who tend to deprecate or downgrade themselves have a general negative self-image and a low sense of mastery. This state of mind may influence health through passivity in the face of disease or neglect of the basics of health care. Guided by this theory we investigate whether items reflecting a low sense of mastery (like:"I can do much for my health") or fatalism ("Health is a consequence of good luck") can explain the association of selfrated health with mortality or morbidity.

\section{Assessment of Self-rated Health}

Self-rated health was assessed during the baseline survey by a semantic differential test of "My health" and by two direct questions: "How would you assess your own health?" (in excellent health, in good health, fairly healthy, not very 
healthy, and not healthy at all). This item was labelled as the evaluative question (Question I). The answers to this question were reduced to three categories:

1. good (values 1 and 2)

2. fairly healthy (value 3 )

3. poor (values 4 and 5 ).

The second question was "What do you think of your own health condition compared to that of other men of your age?". This item was labelled as the comparative question (Question II) with the following categories:

1. better

The semantic differential test tries to measure the psychological meaning of a concept. The concept "automobile", for example, has the spontaneous connotations of "speedy, powerful, easy, expensive". After a traffic accident the connotations "dangerous, unsafe" may dominate the meaning of the concept "automobile". The meaning of the concept may also differ between groups (e.g., police, tourists, parents of small children). The semantic differential test is, therefore, a flexible technique used to study what people feel about something and to find out whether these feelings change after an important experience.

Jenkins developed the semantic differential test for health and applied it to differentiate beliefs and feelings about tuberculosis (Jenkins, 1966). Participants were asked to indicate on a seven-point scale where their feelings were with respect to a pair of adjectives (opposites). These pairs are shown in Table 4.7. For example:

\section{MY HEALTH}

$\begin{array}{lllllllll}\text { good } & 1 & 2 & 3 & 4 & 5 & 6 & 7 & \text { bad } \\ \text { weak } & 1 & 2 & 3 & 4 & 5 & 6 & 7 & \text { strong } \\ \text { normal } & 1 & 2 & 3 & 4 & 5 & 6 & 7 & \text { abnormal }\end{array}$

It was observed that a large number of replies to the semantic differential test were incomplete (59\% and $32 \%$ for Kaunas and Rotterdam, respectively). Especially the pair "quick...slow" was left unanswered by many. Therefore this pair was deleted. Subsequently, men who did not reply to only one, two, or three of the remaining 17 pairs of adjectives were given a score equal to the average of those who did reply to the particular pairs. Those who left more than three items unanswered were not included in the analysis. These procedures increased the number of subjects with complete information to 2810 men in Rotterdam and 1521 men from Kaunas.

We factor-analyzed the semantic differential test by common factor modeling with oblique rotation. This resulted in a two-factor solution that was practically identical in both cohorts. Therefore, the final solution was found by combining the cohorts (Table 4.9). The "evaluative factor" (Factor 1) is determined by the items good-bad, strong-weak, worn out-vigorous and abnormal-normal. The "worry factor" (Factor 2) is determined by the items lighthearted-worried, loose-tense and never-often think 
about it. Two factor scores were computed for each individual with the factor loadings of the pooled cohort. Higher scores show that an individual either rates his health as poor or worries about his health.

Table 4.9 Factor loadings based on the semantic differential test.

\begin{tabular}{lrr}
\hline & Factor 1 & Factor 2 \\
Active-passive & 0.63 & 0.10 \\
Good-bad & 0.76 & 0.23 \\
I never think about it-often think about it & 0.14 & 0.60 \\
I can do much about it-I can't do anything about it & 0.41 & -0.02 \\
Strong-weak & 0.72 & 0.17 \\
It is important to me-it is not important to me & 0.29 & -0.28 \\
It is puzzling to me-I understand it well & -0.35 & 0.13 \\
Tough-tender & 0.43 & 0.14 \\
Abnormal-normal & -0.67 & -0.12 \\
Center of my interest-outside my interest & 0.19 & -0.48 \\
It depends on me-it depends on luck & 0.18 & -0.09 \\
Worn out-vigorous & -0.68 & -0.11 \\
Happy-sad & 0.64 & 0.14 \\
At home we never talk about it-often talk about it & 0.06 & 0.41 \\
Lighthearted-worried & 0.13 & 0.69 \\
Loose-tense & 0.27 & 0.61 \\
Nervous-calm & -0.26 & -0.37 \\
Slow-quick & - & -- \\
Percentage of variance accounted for (not rotated) & 26.1 & 8.4 \\
\hline
\end{tabular}

\section{Comparison between Kaunas and Rotterdam}

Figure 4.2 shows that more men in Rotterdam considered themselves in good health than men in Kaunas [mean factor score: -0.25 and 0.47 , respectively, lower scores showing a more positive evaluation: $P=0.00$ (Student's $t$-test)]. They also worried less about their health, as is shown by the mean factor scores: -0.04 for Rotterdam and 0.07 for Kaunas [ $P=0.00$ (Student's $t$-test)].

Tables 4.10 and 4.11 show the frequency distribution of the answers to the direct questions. These tables show remarkable differences between the cohorts, the Kaunas men giving much more negative answers. Of the participants, $16 \%$ in Rotterdam and $63 \%$ in Kaunas rated their health as poor. Thirty-one percent of the Lithuanians and $14 \%$ of the Dutchmen thought their health status was worse than that of other men. In answering the comparative question, the Dutchmen showed a striking preference for the midpoint category ("the same"). A similar phenomenon was observed regarding some items of the JAS (Section 4.4). Apparently the Dutchmen tended to avoid a social comparison. This might give some extra meaning to "deviating" answers. 


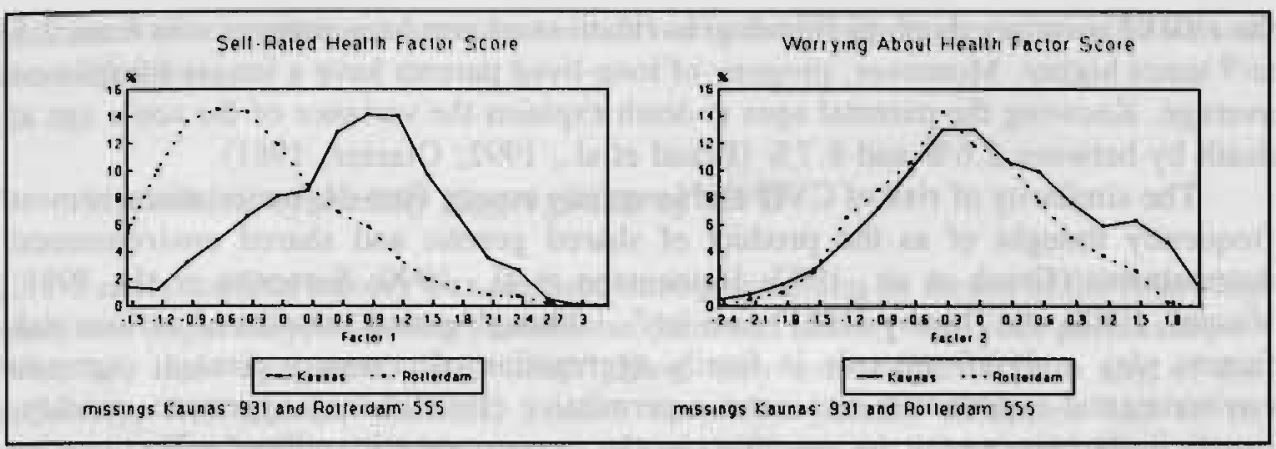

Figure 4.2 Evaluation of and worrying about health in Kaunas and in Rotterdam.

Table 4.10 Question I ("How would you assess your own health?") in Kaunas and in Rotterdam.

\begin{tabular}{lrrrr}
\hline & $\mathrm{N}$ & $\begin{array}{r}\text { Kaunas } \\
\%\end{array}$ & $\mathrm{~N}$ & $\begin{array}{r}\text { Rotterdam } \\
\%\end{array}$ \\
Good & & & 1610 & 51.3 \\
Fairly & 207 & 11.1 & 1033 & 32.9 \\
Poor & 484 & 25.9 & 494 & 15.7 \\
Missing & 1178 & 63.0 & 228 & \\
\hline
\end{tabular}

$P=0.00\left(\right.$ chi $^{2}$ test $)$

Table 4.11 Question II ("How is your health compared to others?") in Kaunas and in Rotterdam.

\begin{tabular}{lrrrr}
\hline & $\mathrm{N}$ & $\begin{array}{r}\text { Kaunas } \\
\text { \% }\end{array}$ & $\mathrm{N}$ & $\begin{array}{r}\text { Rotterdam } \\
\text { \% }\end{array}$ \\
Better & & & 662 & 21.5 \\
Same & 392 & 21.1 & 1974 & 64.2 \\
Worse & 892 & 48.1 & 438 & 14.2 \\
Missing & 570 & 30.7 & 291 & \\
\hline
\end{tabular}

$P=0.00\left(\right.$ chi $^{2}$ test $)$

\subsection{Family Aggregation of CVD}

\section{Research Questions}

An increasing number of studies report on the family clustering of $\mathrm{CHD}$ and the familial similarity in survival (Brand et al., 1992; Colditz et al., 1991; Grech et al., 1992; Vandenbroucke et al., 1984). For example, Slack \& Evans (1966) found that 
the risk of coronary death to first-degree relatives of coronary patients was from 2.5 to 7 times higher. Moreover, progeny of long-lived parents have a longer lifespan on average. Knowing the parental ages at death explains the variance of the son's age at death by between $2.6 \%$ and $8.7 \%$ (Brand et al., 1992; Glasser, 1981).

The similarity of risk of CVD and longevity among first-degree relatives is most frequently thought of as the product of shared genetic and shared environmental determinants (Grech et al., 1992; Heinemann et al., 1990; Sorensen et al., 1988; Vaupel, 1988). As Hamby (1981) puts it: "...although genetic aspects of various risk factors play a significant role in family aggregation of coronary disease, common environmental experiences may exert a permissive effect or may aggravate, modify, or elicit the propensity to manifest in the proper genetic milieu". The precise mechanisms, however, remain ill-defined (Williamson \& Kessling, 1990).

The relative contribution of environmental processes to family clustering of CHD and longevity can best be examined by twin or adoption studies (Sorensen et al., 1988). If such data are not available, investigators must rely on less satisfactory information. This information may be obtained from linking CHD risks and lifespans of individuals who primarily share their environments, such as spouses. Correspondence of disease histories between spouses suggests that environmental factors shared by family members contribute to the familial aggregation of diseases (Kato et al., 1990).

Additional information about environmental processes in family clustering can be gained from sibling aggregation. If two or more siblings have short lifespans or high risks of CHD, while their parents lived to old age or did not have CHD, shared environmental factors may come into prominence. These environmental factors may range from shared early experiences during upbringing to particular health behaviors that are shared by siblings in adulthood and learned from their parents in childhood. It might be that one or both of the parents developed an unhealthy lifestyle later in their lives, such as smoking or consuming too much animal fat. Therefore, although they neither developed CHD nor died prematurely, they nevertheless passed specific habits to the next generation. Furthermore, siblings may have learned specific habits and customs from each other, which may also help us understand processes involved in sibling aggregation. This is not to deny the genetic mechanisms in family aggregation, but sibling aggregation without a parental CHD history at least suggests environmental influences.

It is generally agreed that social position characterizes specific lifestyles within families with sometimes huge impacts on diseases of family members. It is also known that social position is often passed from one generation to the next. Social position may be thought of as a crude environmental exposure shared by family members. One pathway through which disease clusters within families may be the environmental factors shared through reproductive societal processes. In other words, similar social positions of family members result in parents and their offspring having similar risks of CHD or a short lifespan. Furthermore, the social class of the parents may have strong effects on the family environment during upbringing. This may specifically contribute to the sibling clustering of CHD. The KRIS provides an opportunity to 
examine such environmental processes involved in the family aggregation of CHD and short lifespans.

\section{Assessment of Family Aggregation of CVD}

Family medical history was assessed during screening by means of the following questions (separately for mother, father, brothers and sisters): Are your parents alive? What are their ages now, or at death? Have they ever suffered from any of the following: MI, stroke, diabetes, sudden nonviolent death? (Diseases had to be remarked upon separately.) How many of your siblings are living now? How many of your siblings have died? How many of your siblings have suffered from any of the following: MI, stroke, diabetes, sudden nonviolent death? (Diseases had to be remarked upon separately.)

Because of the small numbers in separate analyses of specific events among relatives, MI, stroke, and sudden, nonviolent death were analyzed together. Furthermore, to avoid recall bias in the analysis for possible predictive effects of a CVD family history on the participant's risk of morality or morbidity, KRIS participants with CHD-at-screening were excluded from analysis of these events. The family history of CVD was separately ascertained for parents and siblings. Parental death and ages at death were used to analyze premature death among parents (men with CHD-atscreening were not excluded for these analyses).

\section{Comparison between Kaunas and Rotterdam}

Table 4.12 shows the differences between Kaunas and Rotterdam regarding family histories of CVD; a positive medical family history was more prevalent in Rotterdam. Thirty-two percent of all the men in Kaunas and $39.8 \%$ of all the Dutchmen had a parental history of CVD. Especially having siblings who had experienced a cardiovascular event was more than four times more prevalent in Rotterdam than Kaunas ( $14.1 \%$ and $3.8 \%$, respectively).

In contrast, the number of men whose father or mother was deceased was greater in Kaunas (Table 4.13). Additional analysis of deceased parents showed that the fathers lived almost 6 years longer in Rotterdam - mean age at death: 63.1 in Kaunas and 68.9 in Rotterdam $(P<0.00)$. The lifespans of mothers differed by approximately 4 years - mean age at death: 64.3 in Kaunas and 68.2 in Rotterdam ( $P$ $<0.00)$. 
Chapter 4

Table 4.12 Family aggregation of CVD in Kaunas and in Rotterdam (men with CHD-at-screening excluded).

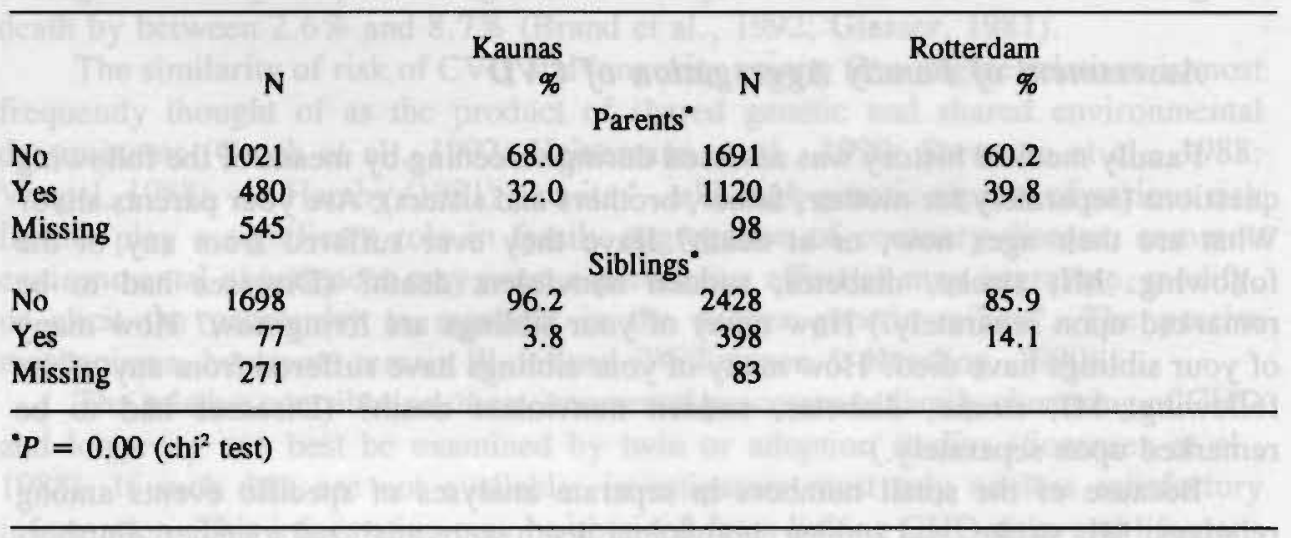

Table 4.13 Parental death in Kaunas and in Rotterdam.

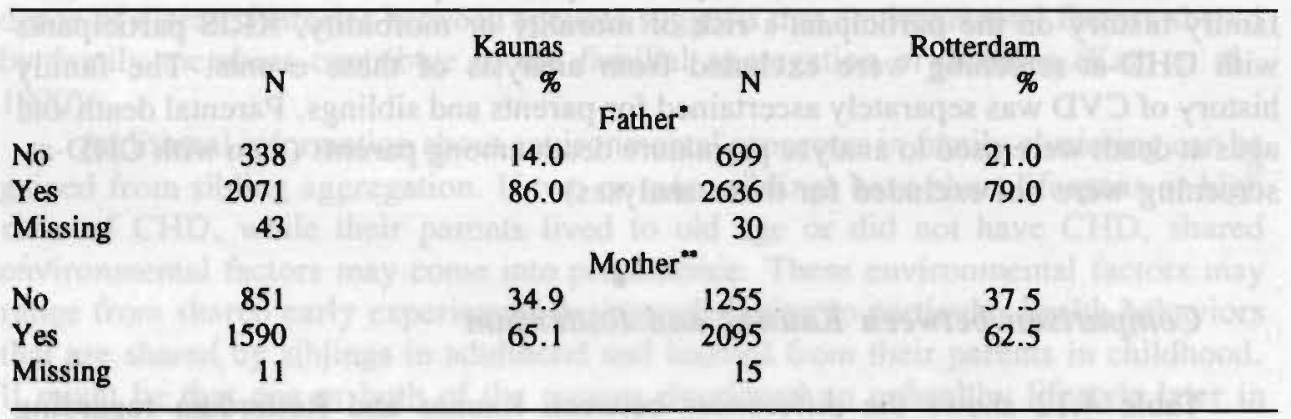

$" P=0.00, " P=0.04$ (chi ${ }^{2}$ test) 
Chapter 5

\section{ANALYSES}

\subsection{Strategy of Analysis}

Since the incidence of angina pectoris, bypass surgery, and strokes in Rotterdam was rather small and since the incidence of these endpoints was not assessed in Kaunas, no attention is given to them. The major endpoints to be studied are:

1. all-cause mortality

2. mortality due to all CVD, including CHD, other heart diseases, and cerebrovascular disease (ICD code: $390-459$ )

3. mortality due to CHD (ICD code: $410-414$ )

4. nonfatal MI.

The analysis starts with the computation of the age-adjusted associations of biological and lifestyle risk factors with the four endpoints. To estimate an adequate, final model for these risk factors, some preliminary analysis had to be done. From a conceptual point of view, both extremely low and extremely high levels of blood pressure, cholesterol, glucose tolerance, and body mass index may be incompatible with life. Therefore the curvilinearity of these risk factors was investigated by introducing a quadratic term to the particular models. We tested whether this interaction term reached the conventional level of statistical significance, i.e., $P<$ 0.05 , to check whether the risks increased if the risk factor deviated in either direction of some point of minimal risk.

Because the systolic blood pressure and the diastolic blood pressure correlated highly in both cohorts, it was decided to include only one of them in the multivariate analysis. Two types of analysis were done to decide which one should be included. One took into account the statistical uncertainty of the coefficients of the association of both variables with an endpoint. The other considered the relative importance of each variable by standardizing the coefficients, i.e., by multiplying them by the standard deviations of the respective variables.

Effect modification by CHD-at-screening was investigated by introducing a product term of this variable and another risk factor to the model with the main terms. These procedures resulted in a final, multivariate model for the biological and lifestyle risk factors.

The analysis of the predictive value of psychosocial risk indicators also begins with adjustment for age. Investigating associations of these risk indicators with biological and lifestyle risk factors was considered informative and worthwhile whenever the age-adjusted association of psychosocial risk indicators with future CVD was substantial. To find out whether given risk factors were important in the etiological pathways relating the risk indicators with the endpoints, the final model 
consisted of the psychosocial risk indicators and all biological and lifestyle risk factors.

If the relative risks of psychosocial risk indicators (hardly) changed after adjusting for these risk factors, this pointed to (partly) independent associations. Decreasing relative risks, particularly to unity, implied that one or more of the biological and lifestyle risk factors could explain the association. Which risk factor or factors were responsible for this decrease was also examined by separately introducing them into the model with age and the particular psychosocial risk indicator.

Did the psychosocial risk indicators also have a predictive value for mortality or nonfatal MI among those with heart disease at screening? The statistical significance of the product term between CHD-at-screening and the psychosocial risk indicator shows to what extent the differences in the predictive value of the psychosocial factor between those with and without CHD-at-screening was due to chance.

\subsection{Statistical Methods}

\section{Mortality}

Most analyses in the following chapters were based on survival analysis. In survival analysis the subjects are followed for varying lengths of time. "Censored observations" are the survival times for individuals for whom no information is available on the occurrence of disease after a specific period. Some survival times may be censored because the particular subject died of a cause other than the cause of interest (competing risks). Other survival times may be censored because the subject left the study before the end of the period of observation. This last type of censoring is known as "lost to follow-up".

Survival analysis is based on survival curves, giving the probability of surviving $t$, conditional on having survived $t-1$. The effect of censored observations is to alter the number of individuals at risk at the next uncensored survival time. An excellent technique for estimating survival curves is the Kaplan-Meier product-limit estimate, which takes advantage of each individual survival time (Kaplan \& Meier, 1958).

Cox (1972) developed a model known as the "proportional hazards model". With this model differences in incidence and mortality risks (actually rates) can be expressed in one parameter, i.e., relative risk or hazard ratio, and several confounders can be taken into account simultaneously. The hazard function represents the instantaneous potential per unit of time for the event to occur, given that the individual has survived until time $t$ (Kleinbaum, 1989). The hazard at time $t, h(t)$, can be expressed as:

$$
h(t)=h_{0}(t) \exp \left(a_{1} X_{1}+a_{2} X_{2}+\ldots a_{n} X_{n}\right) .
$$

In this equation the quantity $h_{0}(t)$ is called the baseline hazard function and it corresponds to the hazards when all variables are zero. The ratio of the estimated 
hazards for different values of a covariate is called the mortality, incidence, or hazard ratio. These ratios are most frequently referred to as relative risks. In the computation of the relative risk we do not need to know the value of the baseline hazard, $h_{0}(t)$. Suppose that the relative risk of mortality for gender must be calculated. Gender is coded as 0 (woman) 1 (man). Then, the relative risk for men compared to women is expressed as:

$$
h_{\text {men }}(t) / h_{\text {women }}(t)=h_{0}(t) \exp \left(a_{1} * 1\right) / h_{0}(t) \exp \left(a_{1} * 0\right)=\exp \left(a_{1}\right)
$$

Because of the proportional hazards assumption in the Cox model, this relative risk is assumed to be equal for each $t$. This assumption explains why the proportional hazards analysis is called a "semiparametric" approach. No particular distribution is assumed for survival times, but the relative risks are assumed to be constant over time.

In the analysis this time independence of relative risk was tested by introducing the product term of the particular covariate and the natural logarithm of time (Kalbfleish \& Prentice, 1980). Whenever the product term was statistically significant, the term is introduced in the model. Whenever the relative risks were not constant during follow-up, it is explicitely mentioned.

In survival analysis men who are lost to follow-up are considered to participate in the follow-up until we have lost sight of them. An important assumption of the Cox model is that the mechanism resulting in loss to follow-up is independent of the mechanism determining survival. For example, loss to follow-up poses a problem when individuals withdraw from follow-up because of physical conditions associated with survival (Cutler \& Ederer, 1958). This subject deserves some special attention because no information was available about the vital status of all the Lithuanians on March 1, 1982, because this was not checked at that date but at a rescreening in 1987. At that time 244 subjects were lost to follow-up because of loss of address or emigration. One should realize that, of all those who were lost to follow-up after the initial screening because of lost addresses, those who died or experienced a nonfatal MI were detected by the mortality and morbidity register. In other words, the chances of detecting the fate of those with unknown addresses were greater for those who became ill or died than for those who stayed healthy. Therefore, censoring was not random, thus violating an assumption of the Cox model. This nonrandom censoring probably resulted in an overestimation of risks in the Kaunas cohort.

To solve this problem, several survival curves were estimated. The different methods of dealing with the 244 men who had been lost to follow-up were:

1. using the last contact date (trail or second screening) as the censoring time point

2. assuming that all 244 men were alive at the end of the follow-up

3. assuming that all 244 men died the day after their last contact date

4. a "halfway" method, which calculated the lost to follow-up date as halfway between the last contact date [screening, second screening (1977-1980), or end of the one-year trial] and June 1987. 
Excepting the third one (which is highly unlikely), only minor differences were observed between these methods. The halfway method probably gives the most accurate estimate of survival (Figure 5.1). As a result, 122 men were no longer considered lost to follow-up before March 1, 1982. The other 122 men did not differ from the rest of the Kaunasians in age, cholesterol, blood pressure, CHD-at-screening, and smoking prevalence.

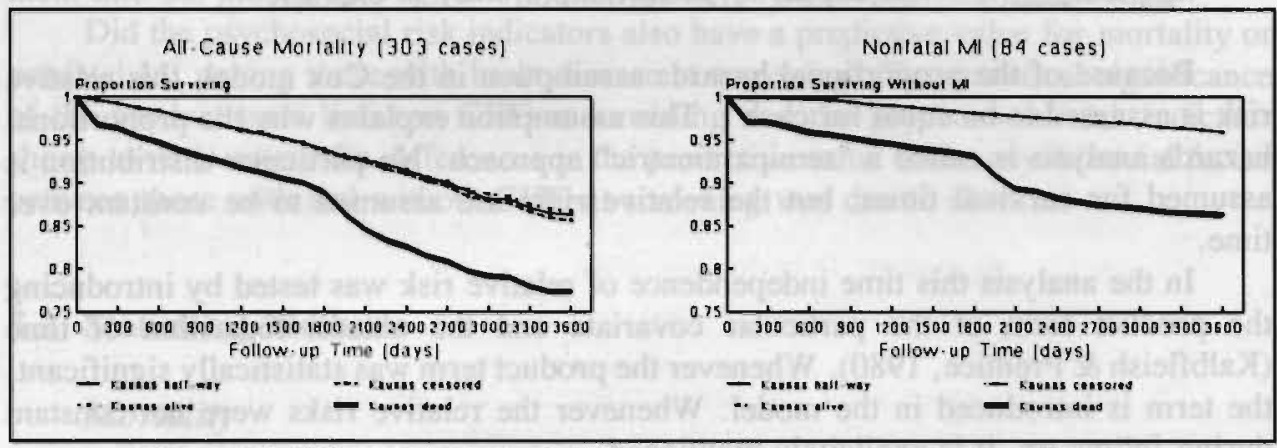

Figure 5.1 Different methods of dealing with lost to follow-up data in Kaunas.

The survival probability, $S(t)$, can be estimated from $\exp (-H(t))$. $H(t)$ is the cumulative hazard, i.e., the cumulative risk of dying between time 0 and $t$. The survival function for any set of covariates is given by:

$$
S(t)=S_{0}(t)^{\exp (\mathbf{s} \mid X 1+22 \times 2+\ldots n n n)} .
$$

In the forthcoming chapters, this formula (with the coefficients of the Cox proportional hazards analysis) sometimes provides an opportunity to draw survival curves for two or more groups when all confounders are, for example, fixed to their mean values. Further details on survival analysis can be found in Altman (1991), Cox \& Oakes (1984), Kalbfleisch \& Prentice (1980), Kleinbaum (1989) and Miller (1981).

\section{Nonfatal MI}

Ascertaining nonfatal MIs at the end of the follow-up in Rotterdam prevented us from counting them in those cases where death had occurred at that time. Furthermore, the date of the MIs was not reported in 38 other cases. In these circumstances, survival analysis is unsatisfactory. Therefore, all analysis involving nonfatal MI in Rotterdam was done with the logistic regression model. In this model probabilities of disease (cumulative incidence rates) can be inferred from:

$$
y=\exp \left(a_{0}+a_{1} X_{1}+\ldots a_{n} X_{n}\right) /\left(\left(1+\exp \left(a_{0}+a_{1} X_{1}+\ldots a_{n} X_{n}\right)\right)\right. \text { or, equivalently, }
$$


$y=1 /\left(1+\exp \left(-\left(a_{0}+a_{1} X_{1}+\ldots a_{n} X_{n}\right)\right)\right.$,

in which $a_{0}, a_{1}$ and $a_{n}$ are coefficients obtained from the logistic regression model. This model corresponds to the logit model:

$\ln (y /(1-y))=a_{0}+a_{1} X_{1}+\ldots a_{n} X_{n}$,

in which $y /(1-y)$ gives the odds for disease.

If $y$ is dependent on a binary variable $X_{1}$, measured as 1 if exposed and 0 if unexposed, then $a_{0}$ corresponds to the logarithm of the odds for disease among the unexposed. Then $a_{0}+a_{1}$ is the logarithm for disease among the exposed. Hence, $a_{1}$ is the difference between the logarithms of the disease odds. The antilogarithm gives the odds for disease among the exposed divided by the odds for disease among the unexposed. This is known as the odds ratio: an estimate of the relative risk. Hosmer \& Lemeshow (1989) and Kleinbaum et al. (1982) provide further details of the model.

In the logistic regression analysis of nonfatal MIs in the Dutch part of the KRIS, those who did not respond to the morbidity questionnaire $(\mathrm{N}=284)$ were excluded. Men who died during the follow-up were also excluded from this analysis. Hence, unless Kaunas and Rotterdam were being compared for risks of nonfatal MI, different techniques were used in each city for the analysis of MI. Comparing results between cohorts was further hampered by differences in diagnostic criteria for nonfatal MI, as presented in Appendix I (Kaunas) and Appendix III (Rotterdam). Therefore, differences between cohorts in results regarding MI should be interpreted cautiously. 


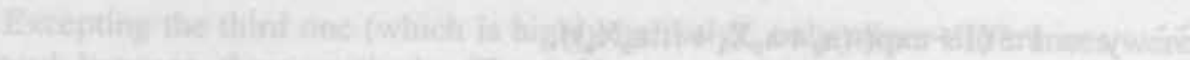

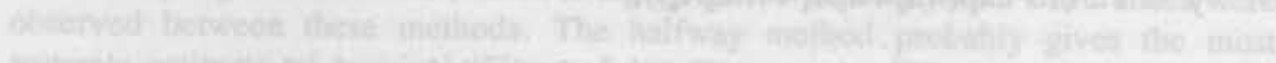

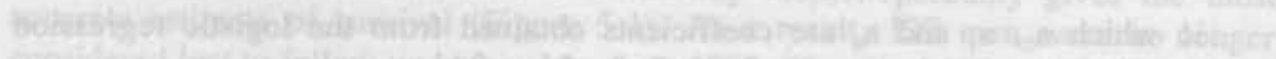

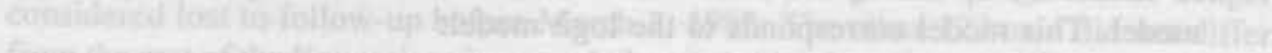

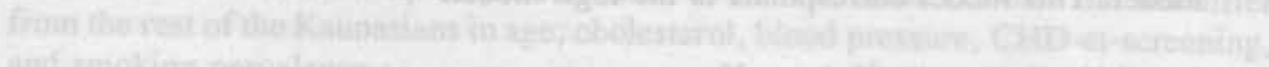

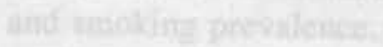

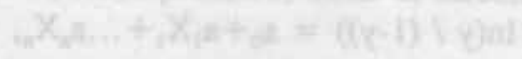

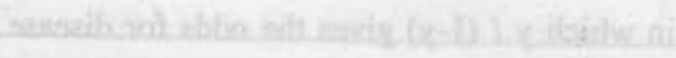

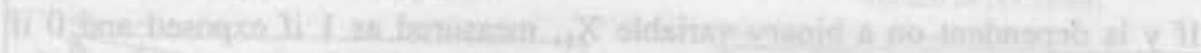

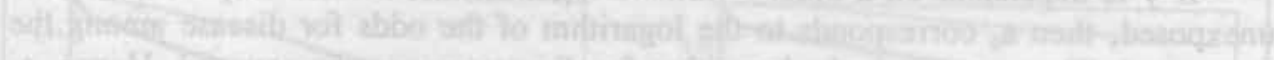

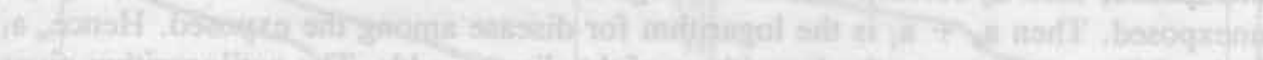

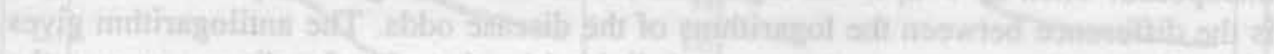

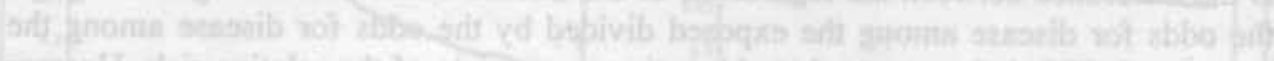

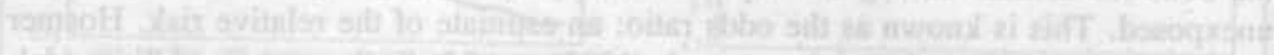

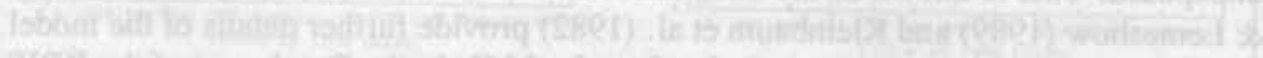

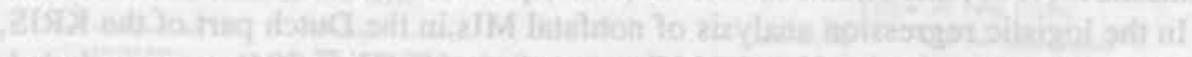

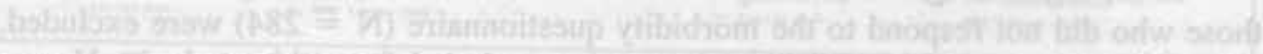

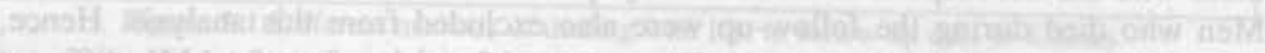

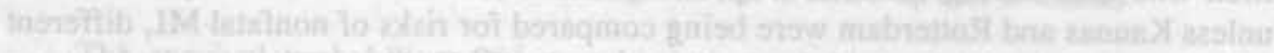

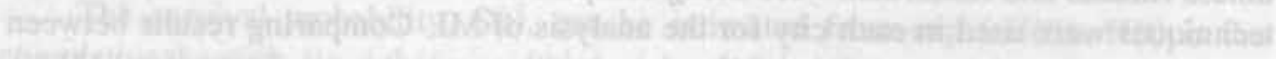

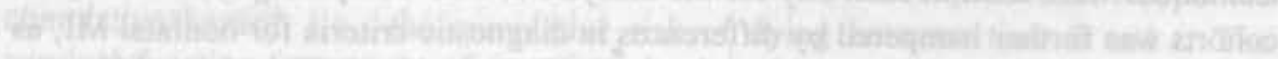

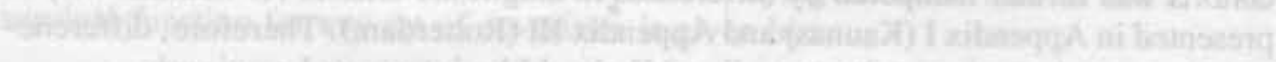

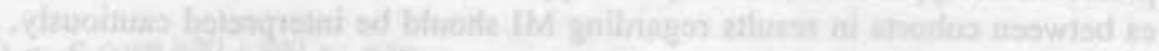

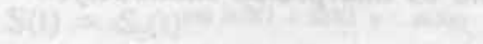

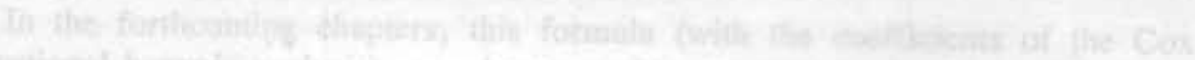

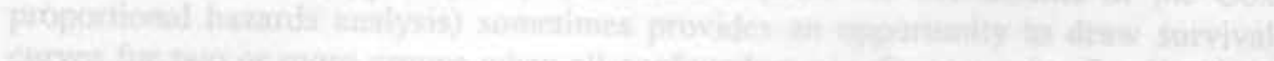

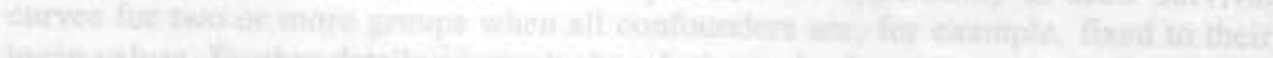

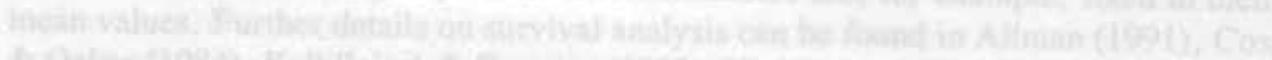

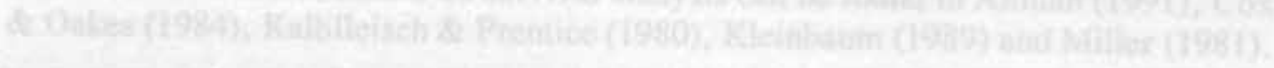

\section{Whaphating}

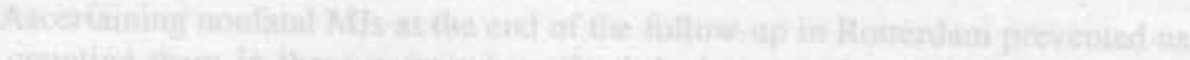

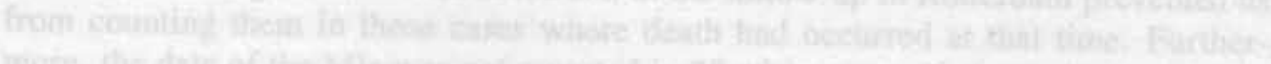

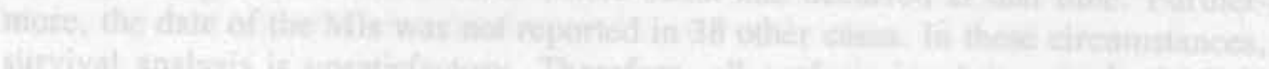

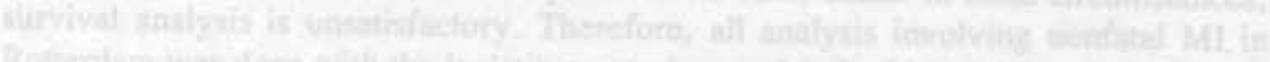

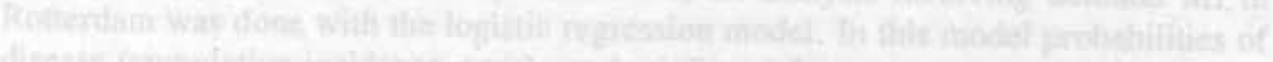

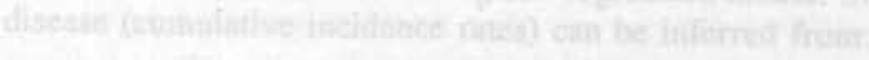

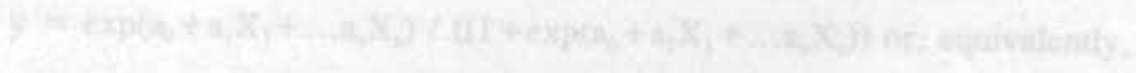


Chapter 6

\section{IMPACT OF BIOLOGICAL AND LIFESTYLE RISK FACTORS}

\subsection{Age-adjusted Models}

\section{CHD-at-screening}

The age-adjusted association of CHD-at-screening with subsequent morbidity and mortality is presented in Table 6.1. As expected in both cohorts, the effects are strong and highly significant. In general, the effects in Kaunas appear somewhat smaller than those in Rotterdam. The relative risks are the highest for mortality due to CVD and $\mathrm{CHD}$, where relative risks in both cohorts show an approximately ninefold mortality risk for men with a definite MI at screening. In the Kaunas cohort, angina pectoris was associated with future nonfatal MI only. The lack of a statistically significant association with the other endpoints might be due to the fact that Kaunas investigators concluded somewhat more readily that angina pectoris was present.

Table 6.1 Age-adjusted association of CHD-at-screening with endpoints.

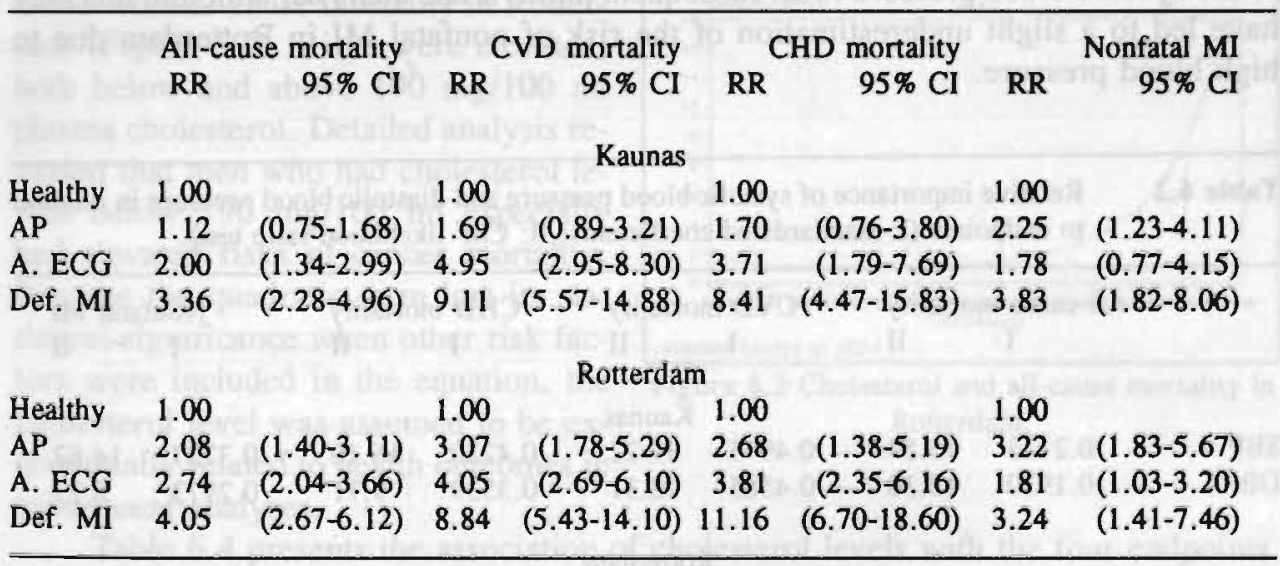

AP, angina pectoris; A. ECG, abnormal ECG; Def. MI, definite MI.

\section{Blood Pressure}

As depicted in Figure 6.1, systolic blood pressure appeared to have a j-shaped association with all-cause mortality in Rotterdam. Mortality risks were elevated, both below and above the minimal hazard point of $129 \mathrm{mmHg}$. The j-shape lost its 
statistical significance when other biological and lifestyle risk factors were included in the equation. In addition, capitalization on chance due to multiple comparisons might have influenced the results. For these two reasons blood pressure was assumed to be exponentially associated with health outcomes.

Given the high correlation between systolic and diastolic blood pressure ( $r$ $=0.70$ in both cohorts), we decided to include only one of them in the multivariate analysis to avoid problems of collinearity. The results of both strategies chosen to find out which was the better predictor are shown in Table 6.2. The table shows that the difference in effect between systolic and diastolic blood pressure on CHD mortality in Rotterdam was too small to prefer one of them unequivocally. Only for nonfatal MI was there an evident preference for diastolic blood pressure, since both the statistical

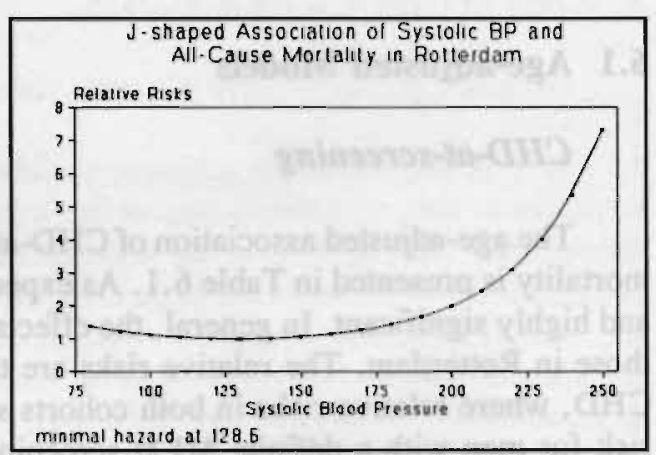

Figure 6.1 Systolic blood pressure and all-cause mortality in Rotterdam. significance and the strength of the effect of the diastolic blood pressure were greater than the effect of the systolic blood pressure. All results in the Kaunas cohort point to systolic blood pressure as the better predictor of survival. Therefore, we decided to use systolic blood pressure in all subsequent multivariate analysis, although this may have led to a slight underestimation of the risk of nonfatal MI in Rotterdam due to high blood pressure.

Table 6.2 Relative importance of systolic blood pressure and diastolic blood pressure in relation to endpoints (I: standardized coefficients; II: $\mathrm{Chi}^{2}$ likelihood ratio test).

$\begin{array}{cccccc}\text { All-cause mortality } & \text { CVD mortality } & \text { CHD mortality } & \text { Nonfatal MI } \\ \text { I } & \text { II } & \text { I } & \text { II } & \text { I II } & \text { I II }\end{array}$

\begin{tabular}{lrrrrrrrr} 
SBP & 0.2133 & 15.54 & 0.4975 & 39.22 & 0.4230 & 16.10 & 0.3737 & 14.62 \\
DBP & 0.1930 & 12.30 & 0.4585 & 28.31 & 0.3539 & 9.71 & 0.2973 & 8.21 \\
& & \multicolumn{8}{c}{ Rotterdam } \\
SBP & 0.1233 & 5.87 & 0.2802 & 14.71 & 0.1381 & 2.54 & 0.1596 & 3.11 \\
DBP & 0.0958 & 3.39 & 0.2512 & 11.44 & 0.1436 & 2.71 & 0.2256 & 6.40
\end{tabular}

The age-adjusted relative risk for systolic and diastolic blood pressure is presented in Table 6.3. The effects of blood pressure were greater in Kaunas. An increase of ten scale points of systolic blood pressure increased the risk of all three 
cardiovascular endpoints by more than $20 \%$ in Kaunas. Blood pressure had less effect on all-cause mortality in both cohorts.

Table 6.3 Age-adjusted association of systolic and diastolic blood pressure with endpoints.

\begin{tabular}{|c|c|c|c|c|c|c|c|c|}
\hline & \multicolumn{2}{|c|}{ All-cause mortality } & \multicolumn{2}{|c|}{ CVD mortality } & \multicolumn{2}{|c|}{ CHD mortality } & \multicolumn{2}{|r|}{ Nonfatal MI } \\
\hline & RR & $95 \% \mathrm{CI}$ & RR & $95 \% \mathrm{CI}$ & RR & $95 \% \mathrm{Cl}$ & RR & $95 \% \mathrm{CI}$ \\
\hline \multicolumn{9}{|c|}{ Kaunas } \\
\hline SBP & 1.12 & $(1.06-1.18)$ & 1.30 & $(1.21-1.40)$ & 1.25 & $(1.13-1.38)$ & 1.22 & $(1.11-1.34)$ \\
\hline DBP & 1.18 & $(1.00-1.29)$ & 1.48 & $(1.29-1.70)$ & 1.35 & $(1.13-1.63)$ & 1.29 & $(1.09-1.53)$ \\
\hline & & & & Rotterdar & & & & \\
\hline SBP & 1.06 & $(1.01-1.12)$ & 1.15 & $(1.07-1.23)$ & 1.07 & $(0.99-1.16)$ & 1.08 & $(0.99-1.18)$ \\
\hline DBP & 1.08 & $(1.00-1.18)$ & 1.23 & $(1.09-1.38)$ & 1.13 & $(0.98-1.29)$ & 1.20 & $(1.05-1.39)$ \\
\hline
\end{tabular}

${ }^{2}$ Relative risks show the increase in risk for an increase of 10 scale points.

\section{Cholesterol}

Cholesterol levels were found to have a statistically significant $\mathrm{j}$-shaped curve with all-cause mortality in Rotterdam (Figure 6.2). Risks were elevated, both below and above $190 \mathrm{mg} / 100 \mathrm{ml}$ plasma cholesterol. Detailed analysis revealed that men who had cholesterol levels below $190 \mathrm{mg} / 100 \mathrm{ml}$ especially had elevated risks of cancer mortality. Because the quadratic term lost its statistical significance when other risk factors were included in the equation, the cholesterol level was assumed to be ex-

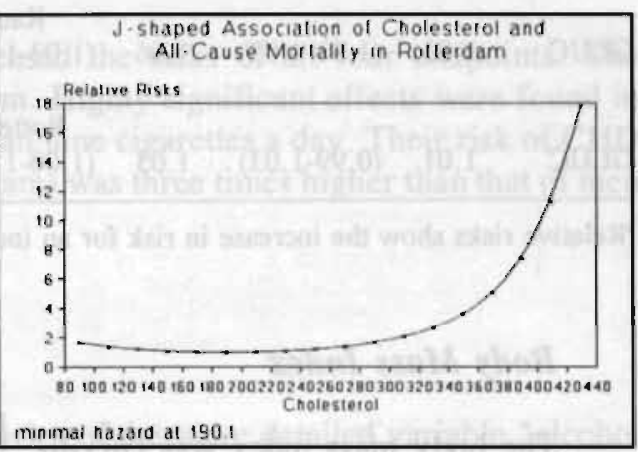

Figure 6.2 Cholesterol and all-cause mortality in Rotterdam. ponentially related to health outcomes in subsequent analyses.

Table 6.4 presents the association of cholesterol levels with the four endpoints. In both cohorts cholesterol levels were associated with the cardiovascular endpoints.

\section{Glucose Tolerance}

Table 6.5 shows the age-adjusted associations of glucose tolerance with the four endpoints. This risk factor was significantly associated with mortality and morbidity 
in Kaunas only. Higher glucose tolerance appeared to be associated with increased risks of the cardiovascular endpoints especially.

Table 6.4 Age-adjusted association of cholesterol with endpoints.

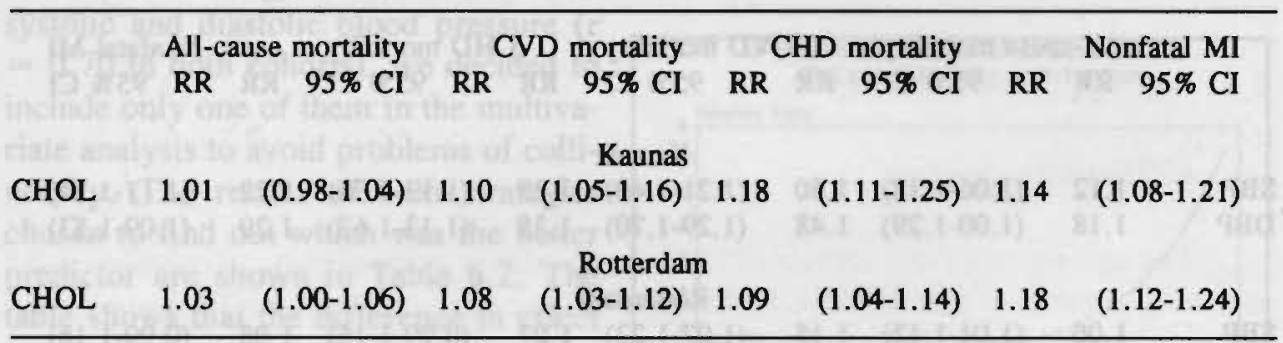

"Relative risks show the increase in the risk for an increase of 10 scale points.

Table 6.5 Age-adjusted association of glucose tolerance with endpoints ${ }^{2}$.

\begin{tabular}{|c|c|c|c|c|c|c|c|c|}
\hline & \multicolumn{2}{|c|}{ All-cause mortality } & \multicolumn{2}{|c|}{ CVD mortality } & \multicolumn{2}{|c|}{ CHD mortality } & \multicolumn{2}{|r|}{ Nonfatal MI } \\
\hline & RR & $95 \% \mathrm{CI}$ & RR & $95 \% \mathrm{CI}$ & RR & $95 \% \mathrm{CI}$ & $\mathbf{R R}$ & $95 \% \mathrm{CI}$ \\
\hline twant & 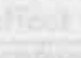 & 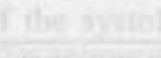 & & Kaunas & & & & \\
\hline GLUC & 1.03 & $(1.01-1.05)$ & 1.06 & $(1.03-1.09)$ & 1.04 & $(1.01-1.09)$ & 1.06 & $(1.03-1.09)$ \\
\hline GLUC & 1.01 & $(0.99-1.03)$ & 1.03 & $\begin{array}{l}\text { Rotterdan } \\
(1.00-1.06)\end{array}$ & 1.02 & $(0.99-1.05)$ & 1.01 & $(0.97-1.04)$ \\
\hline
\end{tabular}

-Relative risks show the increase in risk for an increase of 10 scale points.

\section{Body Mass Index}

The body mass index was curvilinearly associated with all-cause mortality in Kaunas. Figure 6.3 shows that risks were elevated for men who had a body mass index lower or higher than 29. However, for the reasons already mentioned, body mass was treated as a variable that is exponentially related to mortality and morbidity.

As shown in Table 6.6 body mass index was, although not significantly, inversely associated with all-cause mortality. Detailed analysis showed that this

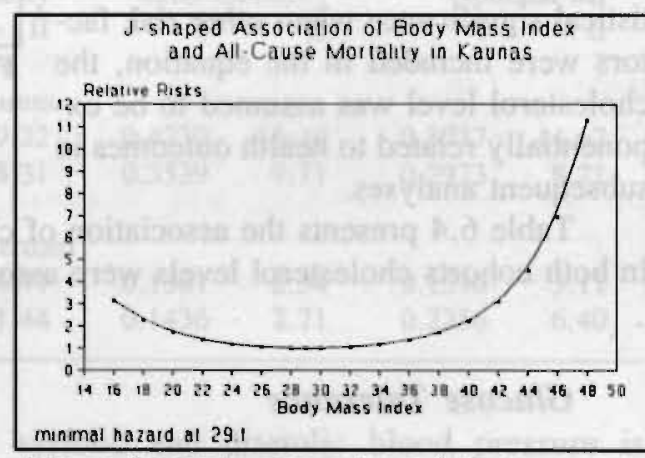

Figure 6.3 Body mass index and all-cause mortality in Kaunas. 
was caused by higher cancer mortality risks among men with a low body mass index. The body mass index was positively and significantly associated with nonfatal MI in both cohorts. Body mass index was also found to increase the risk of CVD and CHD mortality, but the associations reached the conventional level of statistical significance in Rotterdam only.

Table 6.6 Age-adjusted association of body mass index with endpoints ${ }^{2}$.

\begin{tabular}{|c|c|c|c|c|c|c|c|c|}
\hline & \multicolumn{2}{|c|}{ All-cause mortality } & \multicolumn{2}{|c|}{ CVD mortality } & \multicolumn{2}{|c|}{ CHD mortality } & \multicolumn{2}{|r|}{ Nonfatal MI } \\
\hline 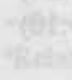 & RR & $95 \%$ CI & RR & $95 \% \mathrm{Cl}$ & RR & $95 \%$ CI & $\mathbf{R} \mathbf{R}$ & $95 \% \mathrm{CI}$ \\
\hline BMI & 0.89 & $(0.65-1.20)$ & 1.57 & $\begin{array}{r}\text { Kaunas } \\
(0.98-2.51)\end{array}$ & 1.68 & $(0.92-3.07)$ & 1.94 & $(1.14-3.31)$ \\
\hline & & & & Rotterdam & & (56) & & \\
\hline BMI & 0.90 & $(0.64-1.27)$ & 1.75 & $(1.08-2.83)$ & 1.86 & $(1.09-3.20)$ & 1.87 & $(1.06-3.29)$ \\
\hline
\end{tabular}

${ }^{2}$ Relative risks show the increase in risk for an increase of 10 scale points.

\section{Smoking}

Table 6.7 shows that smoking increased the risks of all four endpoints. The relative risks tend to be higher in Rotterdam. Highly significant effects were found in both cohorts for men who smoked more than nine cigarettes a day. Their risk of CHD mortality (Kaunas) or nonfatal MI (Rotterdam) was three times higher than that of men who never smoked.

\section{Alcohol Consumption}

Due to small numbers in some categories of the more detailed variable "alcohol consumption", the variable had to be recoded for analysis regarding mortality due to CHD and nonfatal MI. For these endpoints alcohol consumption had to be reduced to a dichotomy: ( 0 ) no alcohol or drinking once a month at the most and (1) drinking unspecified amounts of alcohol at least once a week.

Generally, protective effects of alcohol consumption were found (Table 6.8). The effect of moderate drinking was, however, statistically significant only for Dutch relative risks of all-cause mortality 
Table 6.7 Age-adjusted association of smoking with endpoints.

\begin{tabular}{|c|c|c|c|c|c|c|c|c|}
\hline \multirow{2}{*}{ 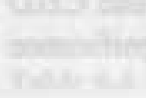 } & \multicolumn{2}{|c|}{ All-cause mortality } & \multicolumn{2}{|c|}{ CVD mortality } & \multicolumn{2}{|c|}{ CHD mortality } & \multicolumn{2}{|r|}{ Nonfatal MI } \\
\hline & RR & $95 \% \mathrm{CI}$ & RR & $95 \% \mathrm{CI}$ & RR & $95 \% \mathrm{CI}$ & RR & $95 \% \mathrm{CI}$ \\
\hline \multicolumn{9}{|c|}{ Kaunas } \\
\hline SMO1 & 1.00 & & 1.00 & & 1.00 & & 1.00 & \\
\hline SMO2 & 1.45 & $(1.01-2.09)$ & 1.29 & $(0.73-2.26)$ & 2.69 & $(1.18-6.15)$ & 1.56 & $(0.82-2.97)$ \\
\hline SMO3 & 1.32 & $(0.80-2.19)$ & 0.85 & $(0.35-2.09)$ & 1.22 & $(0.32-4.61)$ & 1.02 & $(0.38-2.79)$ \\
\hline SMO4 & 2.40 & $(1.66-3.45)$ & 2.19 & $(1.25-3.84)$ & 3.61 & $(1.53-8.51)$ & 1.56 & $(0.75-3.24)$ \\
\hline SMOS & 2.42 & $(1.73-3.39)$ & 1.88 & $(1.10-3.22)$ & 3.82 & $(1.71-8.58)$ & 2.20 & $(1.18-4.10)$ \\
\hline \multicolumn{9}{|c|}{ Rotterdam } \\
\hline SMO1 & 1.00 & & 1.00 & & 1.00 & & 1.00 & \\
\hline SMO2 & 1.96 & $(1.32-4.72)$ & 2.13 & (0.91-5. & 2.63 & $(0.94$ & 3.32 & $(1.01-10.9)$ \\
\hline SMO3 & 2.49 & $(1.32-4.72)$ & 2.24 & $(0.90-5.57)$ & 2.50 & $(0.83-7.54)$ & 3.26 & $(0.92-11.5)$ \\
\hline SMO4 & 3.15 & $(1.74-5.70)$ & 2.52 & $(1.08-5.90)$ & 2.96 & $(1.06-8.31)$ & 4.53 & (1.38-14.8) \\
\hline SMO5 & 2.41 & $(1.32-4.41)$ & 2.10 & $(0.89-4.97)$ & 2.23 & $(0.78-6.37)$ & 4.22 & $(1.28-13.9)$ \\
\hline
\end{tabular}

SMO1, men who never smoked (reference category); SMO2, stopped smoking; SMO3, smoked 1 9 cigarettes a day; SMO4, smoked 10 - 19 cigarettes a day; SMO5, more than 20 cigarettes a day.

Table 6.8 Age-adjusted association of alcohol consumption with endpoints.

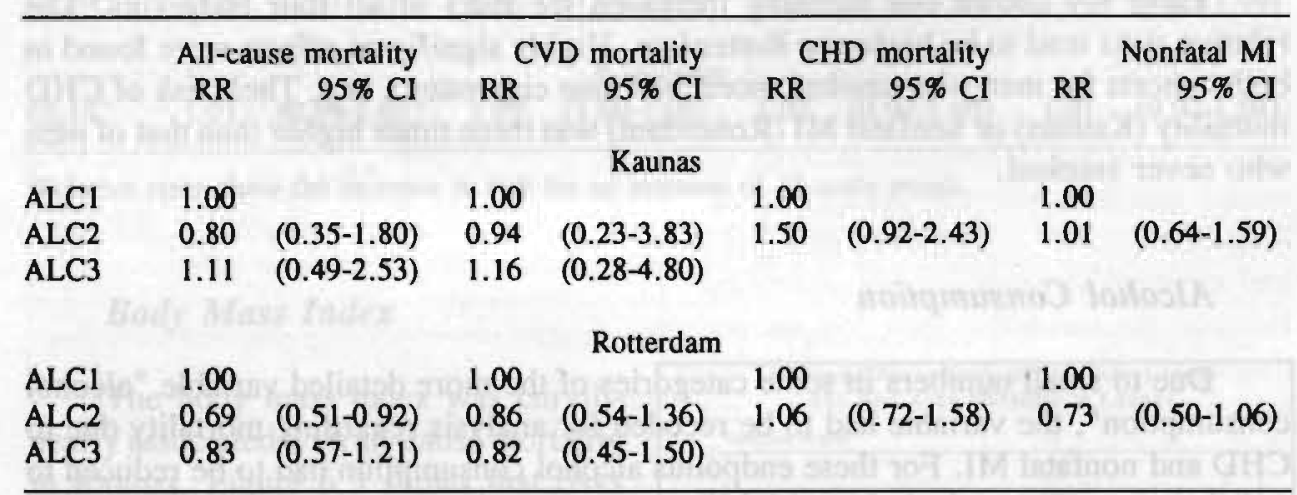

ALC1, no drinking (reference category); ALC2, moderate drinking; ALC3, immoderate drinking. For mortality due to CHD and nonfatal MI the reference category is: ALC1, no alcohol or drinking once a month at the most; ALC2, drinking unspecified amounts of alcohol at least once a week.

\section{Physical Activity}

As Table 6.9 shows, physical activity had only a very small effect. This effect was significant only for all-cause mortality in Kaunas, where risks decreased by approximately $20 \%$ when the physical activity scale increased by 100 units. 
Table 6.9 Age-adjusted association of physical activity with endpoints.

\begin{tabular}{|c|c|c|c|c|c|c|c|c|}
\hline & \multicolumn{2}{|c|}{ All-cause mortality } & \multicolumn{2}{|c|}{ CVD mortality } & \multicolumn{2}{|c|}{ CHD mortality } & \multicolumn{2}{|r|}{ Nonfatal MI } \\
\hline & RR & $95 \% \mathrm{CI}$ & RR & $95 \% \mathrm{Cl}$ & $\mathbf{R R}$ & $95 \% \mathrm{CI}$ & RR & $95 \% \mathrm{CI}$ \\
\hline & MAt & anditis & & & avpl & $615=$ & 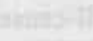 & \\
\hline & & & & Kaunas & & & $a^{2}$ & \\
\hline PHA & 0.81 & $(0.68-0.97)$ & 0.79 & $(0.59-1.06)$ & 0.89 & $(0.62-1.28)$ & 0.98 & $(0.72-1.34)$ \\
\hline hita & 61216 & 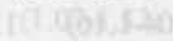 & 1)14:65 & 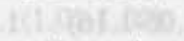 & 1)thly & 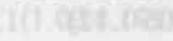 & 1) 1100 & f(chen $19 x)$ \\
\hline & & & & Rotterdam & & & 15 & 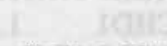 \\
\hline PHA & 0.94 & $(0.74-1.20)$ & 0.79 & $(0.54-1.14)$ & 0.78 & (0.51-1.19) & 0.84 & $(0.54-1.30)$ \\
\hline
\end{tabular}

${ }^{2}$ Relative risks show the increase in risk for the increase of 100 scale points.

\subsection{Multivariate Models}

\subsubsection{Effects of Risk Factors Simultaneously Adjusted}

In the multivariate analysis some categories of CHD-at-screening had to be combined, since the numbers in the more detailed categories were too small to analyze. The new variable had two categories: (0) no CHD-at-screening; (1) CHD-atscreening (definite MI, abnormal ECG deviations, and/or angina pectoris).

Tables 6.10 (Kaunas) and 6.11 (Rotterdam) present the multivariate adjusted effects of all biological and lifestyle risk factors. No large differences were found between the age-adjusted and the multivariate adjusted effects. Most frequently the relative risks decreased somewhat. Two exceptions are noteworthy: smoking and body mass index. The relative risks for smoking increased sharply sometimes, especially in Rotterdam. Detailed analysis shows that this is due to a negative association of smoking with blood pressure and body mass index. Furthermore, the prevalence of smoking among those with CHD-at-screening was lower than among those free of CHD-at-screening. The latter effect was due to those who stopped smoking after a MI. The positive age-adjusted effects of body mass index on CVD mortality, CHD mortality, and nonfatal MI decreased considerably in Kaunas. The gradient of the effects on CVD and CHD mortality even reversed to a negative effect when multivariately adjusted. This was due to the relatively strong association of body mass index with systolic blood pressure ( $r=0.30$ in Kaunas and 0.19 in Rotterdam). 
Table 6.10 Association of all biological and lifestyle risk factors with endpoints in KAUNAS, simultaneously adjusted: $\mathrm{N}=2351$; all-cause mortality: 288 cases; CVD mortality: 110 cases; CHD mortality: 66 cases; nonfatal MI: 81 cases.

\begin{tabular}{|c|c|c|c|c|c|c|c|c|}
\hline & \multicolumn{2}{|c|}{ All-cause mortality } & \multicolumn{2}{|c|}{ CVD mortality } & \multicolumn{2}{|c|}{ CHD mortality } & \multicolumn{2}{|r|}{ Nonfatal MI } \\
\hline & RR & $95 \% \mathrm{CI}$ & RR & $95 \% \mathrm{CI}$ & RR & $95 \%$ CI & RR & $95 \% \mathrm{CI}$ \\
\hline AGE & 1.09 & $(1.06-1.12)$ & 1.11 & $(1.06-1.16)$ & 1.10 & (1.04-1.16) & 1.06 & $(1.01-1.12)$ \\
\hline CHDI & 1.00 & & 1.00 & & 1.00 & & 1.00 & \\
\hline CHD2 & 1.93 & $(1.48-2.53)$ & 3.92 & $(2.64-5.80)$ & 3.11 & $(1.86-5.20)$ & 2.06 & $(1.26-3.36)$ \\
\hline SBP & 1.10 & $(1.04-1.17)$ & 1.23 & $(1.13-1.34)$ & 1.18 & (1.05-1.31) & 1.14 & $(1.03-1.27)$ \\
\hline $\mathrm{CHOL}$ & 1.01 & $(0.97-1.04)$ & 1.08 & $(1.03-1.14)$ & 1.15 & $(1.09-1.22)$ & 1.13 & $(1.07-1.19)$ \\
\hline GLUC & 1.03 & $(1.01-1.05)$ & 1.04 & $(1.01-1.08)$ & 1.03 & $(0.99-1.08)$ & 1.05 & $(1.01-1.09)$ \\
\hline SMOI & 1.00 & & 1.00 & & 1.00 & & 1.00 & \\
\hline $\mathrm{SMO} 2$ & 1.25 & $(0.85-1.82)$ & 1.02 & $(0.57-1.82)$ & 2.14 & $(0.92-4.99)$ & 1.48 & $(0.77-2.84)$ \\
\hline SMO3 & 1.35 & $(0.81-2.25)$ & 0.82 & $(0.33-2.03)$ & 1.21 & $(0.32-4.58)$ & 1.11 & $(0.40-3.04)$ \\
\hline SMO4 & 2.46 & $(1.68-3.61)$ & 2.60 & $(1.44-4.67)$ & 4.24 & $(1.76-10.2)$ & 1.83 & $(0.85-3.93)$ \\
\hline SMO5 & 2.39 & $(1.67-3.42)$ & 1.98 & $(1.13-3.46)$ & 4.07 & $(1.78-9.30)$ & 2.79 & (1.47-5.31) \\
\hline BMI & 0.83 & $(0.60-1.15)$ & 0.80 & $(0.48-1.34)$ & 0.96 & $(0.50-1.85)$ & 1.32 & $(0.73-2.37)$ \\
\hline ALC1 & 1.00 & & 1.00 & & 1.00 & & 1.00 & \\
\hline $\mathrm{ALC} 2$ & 0.63 & $(0.28-1.43)$ & 0.74 & $(0.18-3.04)$ & 1.24 & $(0.75-2.04)$ & 0.87 & $(0.54-1.39)$ \\
\hline ALC3 & 0.81 & $(0.35-1.85)$ & 0.88 & $(0.21-3.67)$ & & & & \\
\hline PHA & 0.84 & $(0.70-1.00)$ & 0.77 & $(0.57-1.03)$ & 0.84 & $(0.58-1.08)$ & 1.02 & $(0.75-1.40)$ \\
\hline
\end{tabular}

CHD1, men without CHD-at-screening (reference category); CHD2, men with CHD-at-screening. Scaling of continuous variables and abbreviations similar to the ones used in previous tables.

\subsubsection{Smoking Habits}

In the multivariate model, the mortality and incidence risks for men who had stopped smoking were still higher than the risks for men who never smoked (especially for CHD mortality and nonfatal MI in Rotterdam). Therefore, it was important to investigate whether risks decrease as the years of no smoking increase. Adjusting for age, it was found that each smoking-free year decreased the risks by approximately $1-1.5 \%(R R=0.99-0.985)$. We take the MI relative risk in Kaunas $(R R=1.48$ in Table 6.10) as an example. The difference in MI incidence in Kaunas between those who had never smoked and ex-smokers would only disappear if the smokers refrained from smoking for more than 25 years $\left(1.48^{-1}=0.985^{x}: x=26\right)$. The effect of the number of years of no smoking is, however, not statistically significant.

Additional analysis revealed that whether one inhaled smoke or not was not associated with mortality or morbidity. The number of years of smoking was not significantly associated with disease either. The age-adjusted relative risk pointed out that the risks increased by approximately $1.5 \%$ a year. 
Table 6.11 Association of all biological and lifestyle risk factors with endpoints in ROTTERDAM, simultanenusly adjusted: $\mathrm{N}=3221$; all-cause mortality: 340 cases; CVD mortality: 157 cases; CHD mortality: 122 cases; nonfatal MI: 123 cases.

\begin{tabular}{lccccccccc}
\hline & \multicolumn{2}{c}{ All-cause mortality } & \multicolumn{2}{c}{ CVD mortality } & \multicolumn{2}{c}{ CHD mortality } & Nonfatal MI \\
& RR & $95 \%$ CI & RR & $95 \%$ CI & RR & 95\% CI & RR & 95\% CI \\
AGE & 1.10 & $(1.07-1.13)$ & 1.05 & $(1.01-1.09)$ & 1.04 & $(1.00-1.09)$ & 1.05 & $(1.00-1.10)$ \\
CHD1 & 1.00 & & 1.00 & & 1.00 & & 1.00 & \\
CHD2 & 2.60 & $(2.06-3.29)$ & 3.97 & $(2.85-5.52)$ & 4.21 & $(2.89-6.12)$ & 2.33 & $(1.49-3.64)$ \\
SBP & 1.05 & $(1.00-1.11)$ & 1.07 & $(1.02-1.18)$ & 1.01 & $(0.93-1.10)$ & 1.02 & $(0.93-1.12)$ \\
CHOL & 1.02 & $(0.99-1.05)$ & 1.06 & $(1.01-1.10)$ & 1.06 & $(1.01-1.11)$ & 1.17 & $(1.11-1.23)$ \\
GLUC & 1.01 & $(0.99-1.03)$ & 1.03 & $(1.01-1.06)$ & 1.03 & $(0.99-1.06)$ & 1.02 & $(0.98-1.05)$ \\
SMO1 & 1.00 & & 1.00 & & 1.00 & & 1.00 & \\
SMO2 & 2.02 & $(1.10-3.71)$ & 2.32 & $(0.99-5.44)$ & 2.71 & $(0.96-7.63)$ & 3.24 & $(0.97-10.8)$ \\
SMO3 & 2.50 & $(1.31-4.77)$ & 2.56 & $(1.02-6.43)$ & 2.68 & $(0.88-8.16)$ & 3.63 & $(1.01-13.1)$ \\
SMO4 & 3.28 & $(1.80-5.98)$ & 2.99 & $(1.26-7.09)$ & 3.43 & $(1.21-9.74)$ & 5.37 & $(1.61-17.9)$ \\
SMO5 & 2.57 & $(1.40-4.72)$ & 2.53 & $(1.06-6.02)$ & 2.52 & $(0.88-7.23)$ & 4.66 & $(1.40-15.5)$ \\
BMI & 0.94 & $(0.65-1.36)$ & 1.47 & $(0.87-2.47)$ & 1.74 & $(0.97-3.14)$ & 1.70 & $(0.91-3.17)$ \\
ALC1 & 1.00 & & 1.00 & & 1.00 & & 1.00 & \\
ALC2 & 0.71 & $(0.52-0.95)$ & 0.91 & $(0.56-1.46)$ & 1.11 & $(0.74-1.66)$ & 0.73 & $(0.49-1.08)$ \\
ALC3 & 0.82 & $(0.56-1.20)$ & 0.83 & $(0.45-1.52)$ & & & & \\
PHA & 0.97 & $(0.75-1.24)$ & 0.92 & $(0.63-1.34)$ & 0.88 & $(0.58-1.35)$ & 0.89 & $(0.57-1.40)$ \\
\hline
\end{tabular}

Scaling of continuous variables and abbreviations similar to the ones used in previous tables.

\subsubsection{Effect Modification by CHD-at-screening}

As argued in Chapter 5, we did not take CHD-at-screening into account by excluding all those with signs of possible or documented CHD-at-screening from the analysis, but by including it as a biological risk factor in the multivariate models. The effect modification of this risk factor was investigated by introducing the product term between CHD-at-screening and each risk factor into the multivariate model. A separate analysis was done for each risk factor. Two statistically significant product terms were observed. Although these interactions were statistically significant in one cohort only, the direction of the coefficients is the same in the other cohort. Table 6.12 presents the results. Higher glucose tolerance was associated with higher mortality and morbidity risks, but the increase in risks was higher for those men with indications of CHD-at-screening. An increase of glucose tolerance (ten scale points) for those without CHD-at-screening was associated with a $1 \%$ (in Rotterdam) and a $3 \%$ (in Kaunas) increase in mortality risks due to CVD. The increase for CHD-prevalent cases was $8 \%$ and $6 \%$, respectively. Similarly, the effect of physical activity was especially beneficial for CHD-prevalent cases, but only for all-cause mortality. The relative risks of other risk indicators did not change substantially compared to the model without interactions. 
Table 6.12 Age-adjusted association of glucose tolerance and physical activity with endpoints, separately for prevalent and nonprevalent cases.

\begin{tabular}{|c|c|c|c|c|c|c|c|c|}
\hline & \multicolumn{2}{|c|}{ All-cause mortality } & \multicolumn{2}{|c|}{ CVD mortality } & \multicolumn{2}{|c|}{ CHD mortality } & \multicolumn{2}{|r|}{ Nonfatal MI } \\
\hline 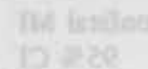 & RR & $95 \% \mathrm{CI}$ & RR & $95 \% \mathrm{CI}$ & RR & $95 \% \mathrm{CI}$ & RR & $95 \% \mathrm{CI}$ \\
\hline \multicolumn{9}{|c|}{ Kaunas } \\
\hline GLUC & 1.02 & $(0.99-1.05)$ & 1.03 & $(0.99-1.08)$ & 1.01 & $(0.95-1.07)$ & 1.01 & $(0.96-1.06)$ \\
\hline PHA & 0.92 & $(0.76-1.12)$ & & & & & & \\
\hline & $x_{1}^{2}$ & & & Prevalent cas & & & . & \\
\hline GLUC & 1.05 & $(1.02-1.08)$ & 1.06 & $(1.01-1.11)$ & 1.07 & $(1.01-1.14)$ & 1.12 & $(1.05-1.19)$ \\
\hline PHA & 0.62 & $(0.42-0.90)$ & & & & & & \\
\hline 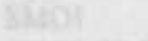 & $1 / 405$ & & 1890 & & 1200 & & & \\
\hline stivingeres & $0 x+3 y$ & & & Rotterdam & & & & \\
\hline thowitis & & & & Vonprevalent & & & & 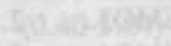 \\
\hline GLUC & 0.99 & $(0.97-1.02)$ & 1.01 & $(0.97-1.05)$ & 0.98 & $(0.94-1.03)$ & 1.01 & $(0.97-1.05)$ \\
\hline PHA & 1.06 & $(0.78-1.42)$ & & $1 x^{2}=2$ & & & & \\
\hline 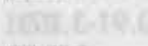 & 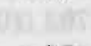 & 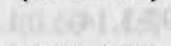 & & Prevalent cas & & & & \\
\hline GLUC & 1.05 & $(1.03-1.07)$ & 1.08 & $(1.04-1.13)$ & 1.10 & $(1.04-1.16)$ & 1.06 & $(0.98-1.14)$ \\
\hline PHA & 0.83 & $(0.52-1.31)$ & & & & & & \\
\hline
\end{tabular}

Scaling of continuous variables and abbreviations similar to the ones used in previous tables.

The high relative risks among CHD-prevalent cases may be based on seriousness of CHD-at-screening. Although no detailed information was available about it, we decided to approximate the seriousness of CHD with the categories angina pectoris, abnormal ECG, and definite MI. Among CHD-prevalent cases, the effect of glucose tolerance and physical activity did not decrease when this crude approximation of seriousness of CHD was taken into account. Furthermore, the effect of both risk indicators was calculated for the separate categories of CHD. The effect in each category was greater than the effect for men without indications of CHD.

\subsection{Changes in Risk Factor Levels}

All the associations between the biological risk factors and future morbidity and mortality just presented were based upon the assessment of these risk factors at entry. It is, however, interesting to see if changing risk factor levels influenced the relative risk of all-cause mortality as well as the mortality caused by CVD and CHD. Because the Lithuanians were invited for a five-year follow-up examination in 1977-1980, it was possible to investigate this question in the Lithuanian cohort.

All subjects who participated in the initial screening examination were invited for the five-year follow-up examination. This invitation was accepted by $1353(55 \%)$ of the subjects. Of this group 72 subjects died during the following years, of them 26 due to CVD and 17 due to CHD. 
Table 6.13 presents the correlations of the risk factor levels at screening and the change in these levels. The table shows that a change in diastolic blood pressure was related to the initial level of blood pressure and (weakly) to the initial level of cholesterol and body mass index. Separate analyses for subjects with and without CHD-at-screening showed that changes in systolic blood pressure and body mass index did not differ in these groups. However, the cholesterol level increased in cases without CHD, but notably decreased in those with CHD-at-screening.

Table 6.13 Pearson correlation coefficients between risk factor levels at initial screening and their changes over a five-year period (Kaunas).

\begin{tabular}{lcccc}
\hline & $\delta S B P$ & $\delta$ DBP & $\delta$ CHOL & $\delta$ BMI \\
AGE & 0.04 & 0.06 & -0.02 & -0.01 \\
SBP & $-0.30^{* *}$ & $-0.24^{* *}$ & -0.04 & 0.05 \\
DBP & $-0.13^{* *}$ & $-0.41^{* *}$ & $-0.07^{* *}$ & 0.01 \\
CHOL & -0.02 & $-0.06^{*}$ & $-0.40^{* *}$ & 0.04 \\
BMI & 0.03 & $-0.07^{*}$ & $-0.07^{*}$ & $-0.18^{* *}$ \\
$\delta$ SBP & & $0.62^{* *}$ & $0.06^{*}$ & $0.09^{* *}$ \\
$\delta$ DBP & & & $0.12^{* *}$ & $0.12^{* *}$ \\
$\delta$ CHOL & & & & $0.10^{* *}$ \\
\hline
\end{tabular}

Abbreviations similar to the ones used in previous tables.

$" P<0.05, " P<0.01$

We used the Cox proportional hazards analysis to find out if, besides the initial values of the risk factors, the changes in the risk factor levels were also associated with mortality. As shown in Table 6.14 the net changes in systolic blood pressure, diastolic blood pressure, and cholesterol level contributed marginally, but still significantly, to the increased risk of all-cause mortality.

Table 6.14 Association of changes in risk factors in 5 years with endpoints, adjusted for age, systolic blood pressure, diastolic blood pressure, cholesterol, body mass index, and CHD-at-screening.

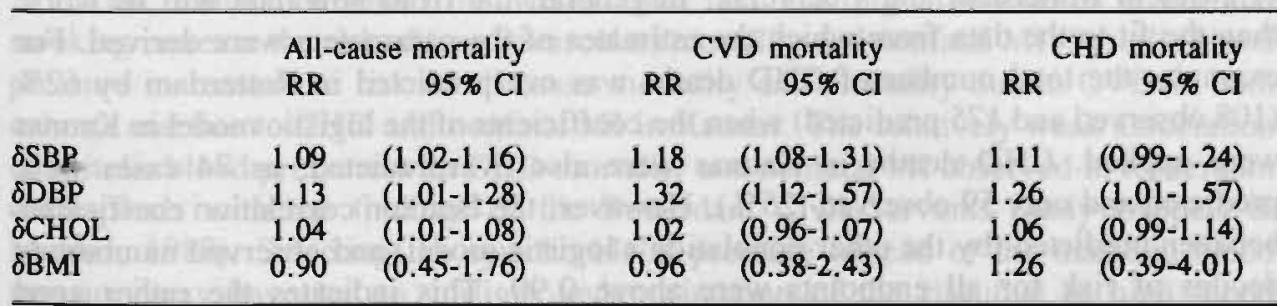

Scaling of continuous variables and abbreviations similar to the ones used in previous tables. 
Similarly, changes in systolic blood pressure and diastolic blood pressure added significantly to the increased risk of CVD mortality. However, only changes in diastolic blood pressure were significant predictors of CHD mortality. In this context, it is important to realize that, partly due to the regression towards the mean phenomenon, the changes correlated negatively with the initial values. This masked the strength of the association of changes (increasing risk factor levels) with mortality when the initial values were not taken into account.

\subsection{Predictive Value of Biological and Lifestyle Risk Factors}

The utility of the models consisting of all biological and lifestyle risk factors can be tested by comparing the estimated or predicted number of cases with the actual number of cases. For each individual a logistic regression solution provided probabilities of an event in the future ${ }^{1}$. These probabilities were based on individual values on all biological and lifestyle risk factors in the logistic regression model. The probabilities were ordered and divided into decile classes. The predicted number of cases in each decile class was calculated by summing the individual probabilities per decile class. For example, for 1000 men with a probability of CHD mortality of 0.01 , it was estimated that 10 would die of CHD. These numbers could, then, be compared with the actual number of cases in these decile classes of risk.

The estimated and observed numbers of cases for all four endpoints are shown in Figures 6.4 and 6.5. The figures show the rather close correspondence between the number of cases observed and the number of cases predicted by the logistic regression function of entry characteristics of all biological and lifestyle risk factors. Figure 6.4 also shows that 81 out of 288 deaths in total $(28 \%)$ occurred in $10 \%$ (the highest risk decile) of the Kaunas population. Corresponding numbers for Rotterdam were 92 out of 340 deaths $(27 \%)$ in the highest risk decile. Prediction and observation corresponded better for the cardiovascular endpoints. Both the Lithuanians and the Dutch men in the highest risk deciles accounted for $35-45 \%$ of all CVD or CHD mortality during follow-up. The prediction of nonfatal MI in Rotterdam was somewhat worse, as only $23 \%$ of all nonfatal MIs occurred in the highest risk decile (35\% in Kaunas).

Parameters of the risk function of Kaunas can also be used to predict the number of cases in Rotterdam and vice versa. In general, the fit to new data will be worse than the fit to the data from which the estimates of the parameters were derived. For example, the total number of CHD deaths was overpredicted in Rotterdam by $62 \%$ (108 observed and 175 predicted) when the coefficients of the logistic model in Kaunas were applied. CHD deaths in Kaunas were also overpredicted, as 74 cases were predicted and only 59 observed ( $25 \%$ ). However, the Pearson correlation coefficients between predicted (by the other population's logistic model) and observed numbers in deciles of risk for all endpoints were above 0.90 . This indicates the rather good

1 Results with the Cox proportional hazards model were essentially the same, but for reasons of computational ease of the logistic regression model not presented. 


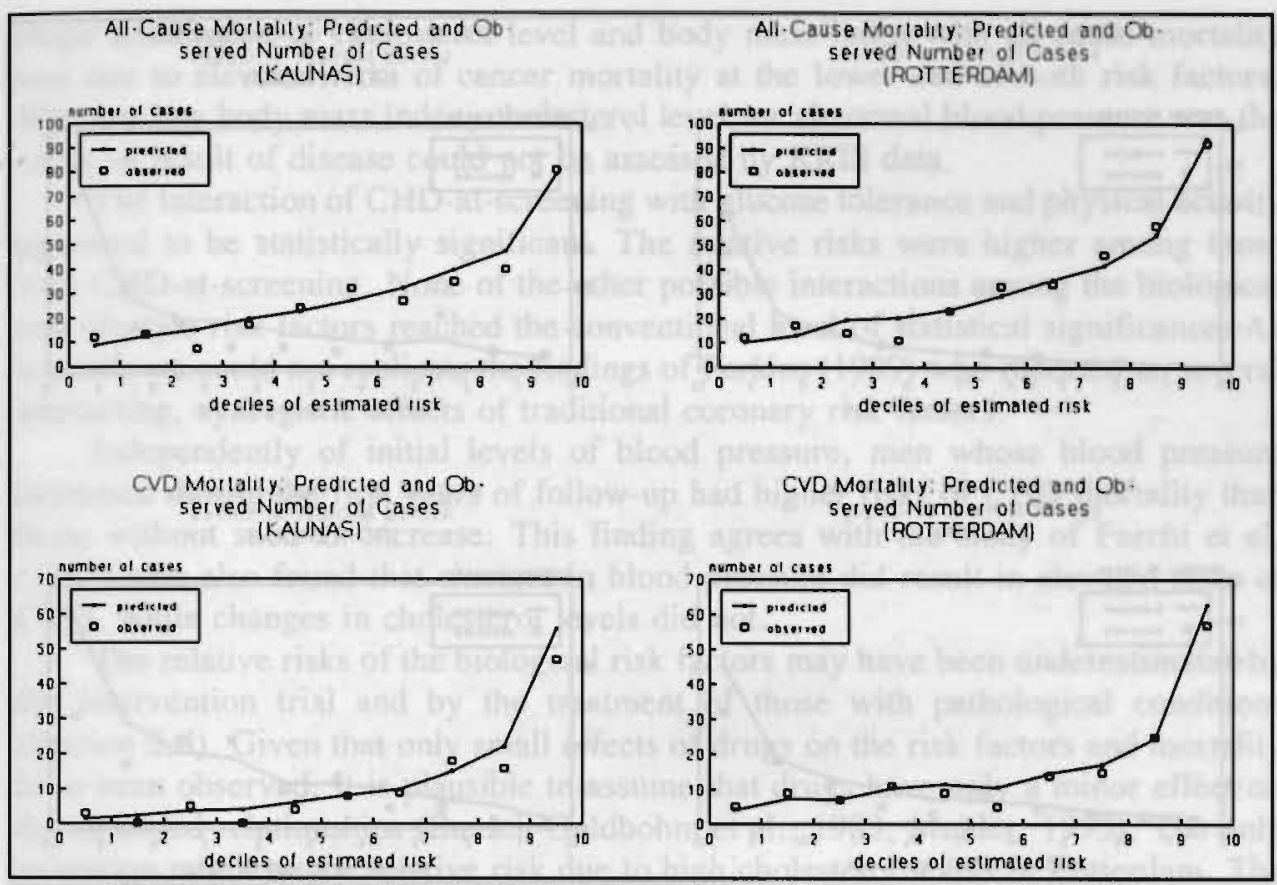

Figure 6.4 Predicted and observed number of cases: all-cause and CVD mortality.

approximation of the relative risk by the logistic model of the other population. For example, $30 \%$ and $34 \%$ of all CHD deaths in Rotterdam and Kaunas, respectively, occurred in the highest risk decile, as estimated from the other population's logistic model. Hence, coefficients of the logistic regression model of Kaunas were capable of a good ranking of the Dutchmen as low, medium, and high-risk subjects and vice versa.

\subsection{Summary and Discussion}

The results confirmed the well-known fact that high cholesterol levels and smoking increase the risk of (cardiovascular) mortality and nonfatal MI. Systolic blood pressure increased the risk of all-cause mortality and mortality due to CVD in both cohorts and due to CHD and nonfatal MI in Kaunas. The relatively weak association of systolic blood pressure with CHD mortality and nonfatal MI observed in Rotterdam corresponds with the findings of the Rotterdam Civil Servants Study (Appels \& Mulder, 1988). Selecting the systolic blood pressure instead of the diastolic blood pressure may have led to a slight underestimation of the risk due to high blood pressure in Rotterdam. An elevated body mass index increased the risk of CHD mortality and nonfatal MI mainly through its association with blood pressure. Except for all-cause mortality in Rotterdam, alcohol consumption was not significantly 


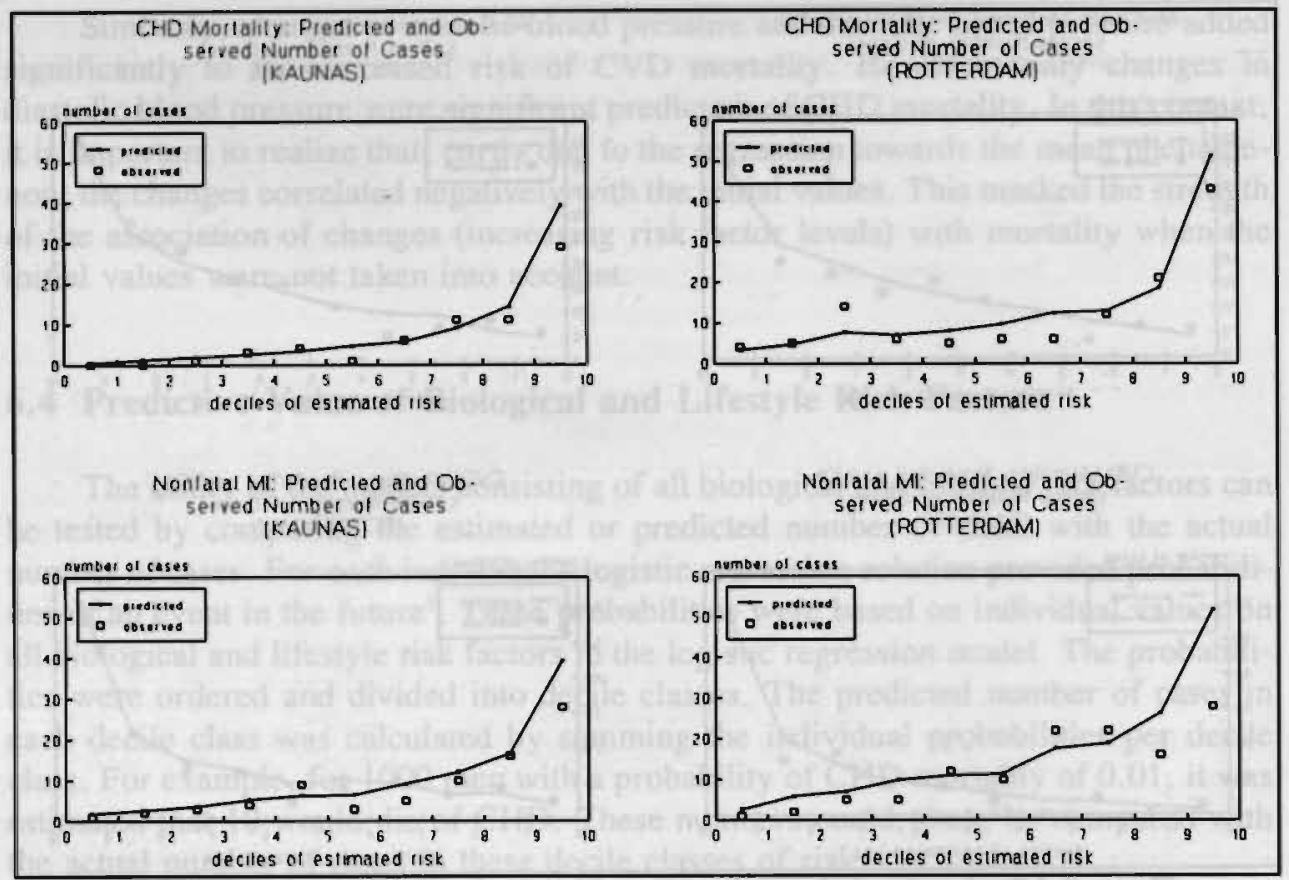

Figure 6.5 Predicted and observed number of cases: CHD mortality and nonfatal MI.

associated with any of the endpoints. The general picture is that moderate alcohol consumption tended to have a health-protecting effect.

Additional analysis of smoking revealed that the number of years of no smoking and the number of years ex-smokers had smoked caused a 1-1.5\% decrease and increase of risks, respectively. For smokers the number of smoking years was also associated with a $1-1.5 \%$ increase in risks. The generally higher relative risk caused by smoking in Rotterdam could, however, not be explained by the longer periods the Dutchmen had smoked. Smoking hand-rolled cigarettes, which was customary for $50 \%$ of all Dutch smokers, had no extra harmful effects. The contribution of the less adverse chemical constituents of USSR cigarettes, as reported by Djordjevic (1991), to the smaller relative risks of smoking in Lithuania is worth further investigation.

In preparing the analysis, much attention was given to the shape of the curve relating the risk factors and the endpoints. A point of minimal risk was computed for systolic blood pressure, cholesterol level, and body mass index. The results suggested that deviations in either direction were associated with higher all-cause mortality risks. This corresponds to the studies of Bray (1987), Hoffmans et al. (1989), Kannel et al. (1986), Lindholm et al. (1986; 1992), Prokhorskas (1992), and Rissanen et al. (1989). A j-shape relating blood pressure, cholesterol level and body mass index to all-cause mortality was also observed in the KRIS. However, exponential associations were assumed because the $\mathrm{j}$-shapes lost their statistical significance when other risk factors were included in the model. It might be important to report that in the KRIS the j- 
shape association of cholesterol level and body mass index with all-cause mortality was due to elevated risks of cancer mortality at the lower end of both risk factors. Whether low body mass index, cholesterol level, or abnormal blood pressure was the cause or result of disease could not be assessed by KRIS data.

The interaction of CHD-at-screening with glucose tolerance and physical activity appeared to be statistically significant. The relative risks were higher among those with CHD-at-screening. None of the other possible interactions among the biological and lifestyle risk factors reached the conventional level of statistical significance. As a result, we could not replicate the findings of Perkins (1989) who reported on several interacting, synergistic effects of traditional coronary risk factors.

Independently of initial levels of blood pressure, men whose blood pressure increased during the first years of follow-up had higher risks of CHD mortality than those without such an increase. This finding agrees with the study of Farchi et al. (1981) who also found that changes in blood pressure did result in elevated risks of CHD, while changes in cholesterol levels did not.

The relative risks of the biological risk factors may have been underestimated by the intervention trial and by the treatment of those with pathological conditions (Section 2.4). Given that only small effects of drugs on the risk factors and mortality have been observed, it is plausible to assume that drugs have only a minor effect on the observed relationships (Bausch-Goldbohm et al., 1983; Mulder, 1993). The only exception might be the relative risk due to high cholesterol levels in Rotterdam. The treatment of a high level of cholesterol by clofibrate resulted in a significant decline of the incidence of MI in the experimental group compared to a control group. The intervention also resulted in a significant increase of cancer mortality in the treatment group (Appels et al., 1989). It is, thus, not unlikely that the effect of cholesterol levels on CVD is somewhat underestimated because of the treatment of those with elevated cholesterol levels. 


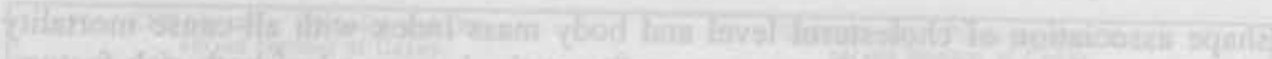

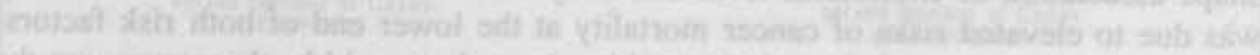

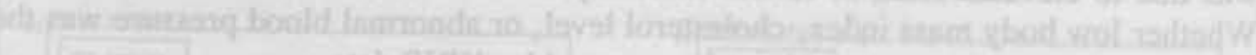

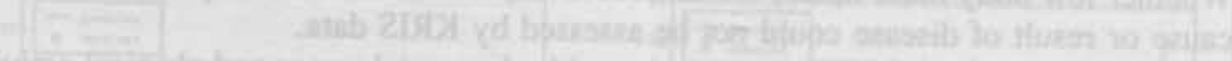

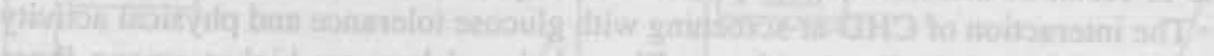

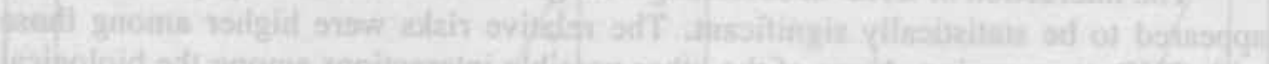

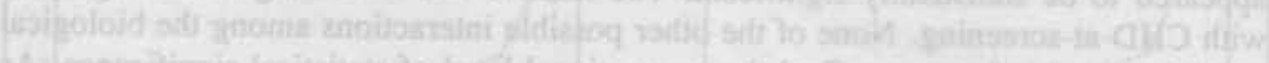
2A

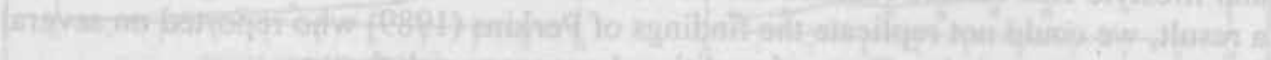

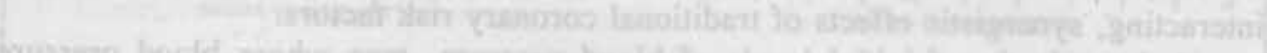

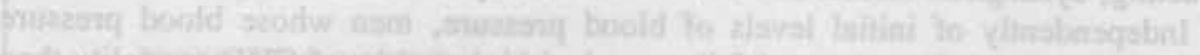

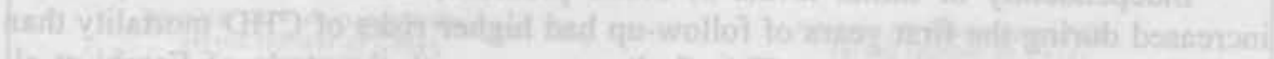

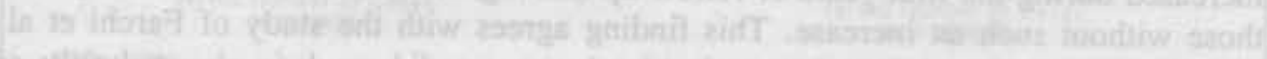

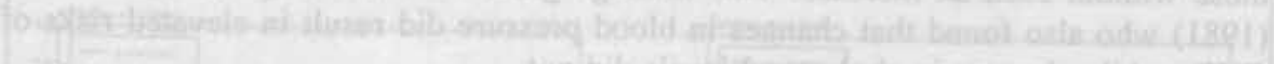

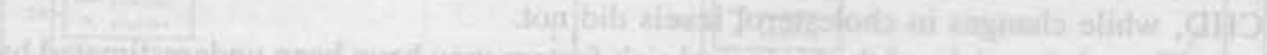

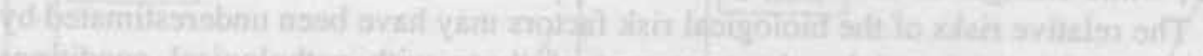

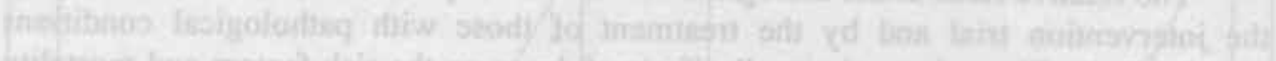

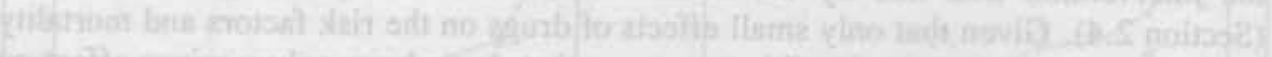

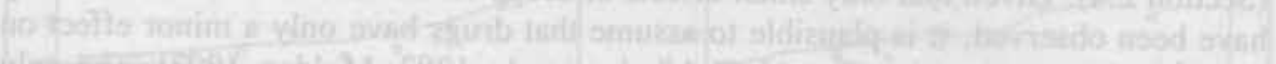

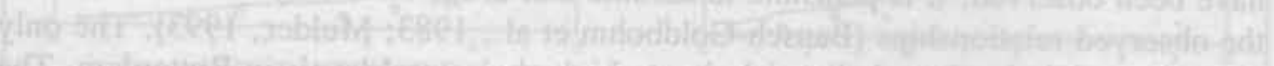

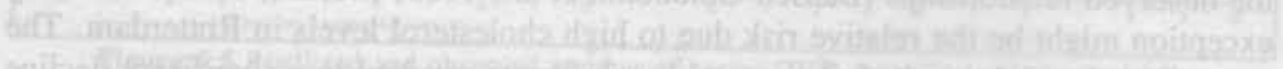

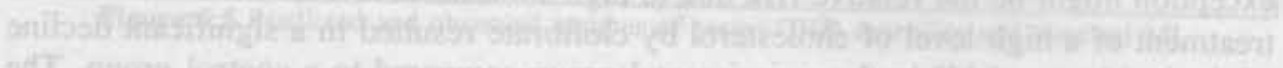

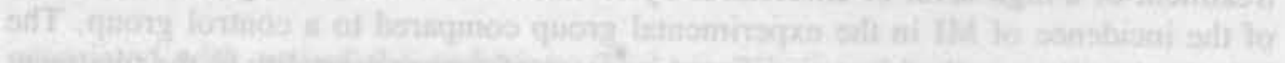

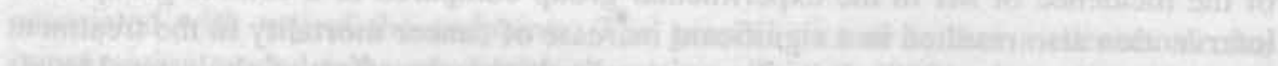

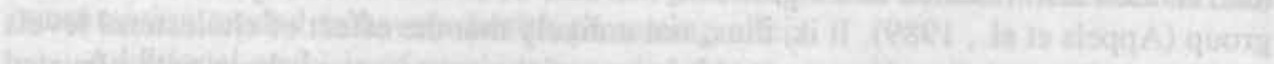

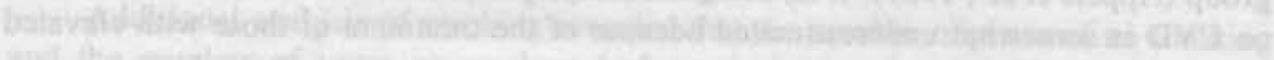

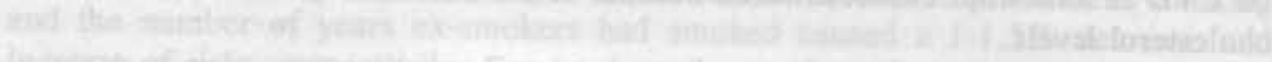

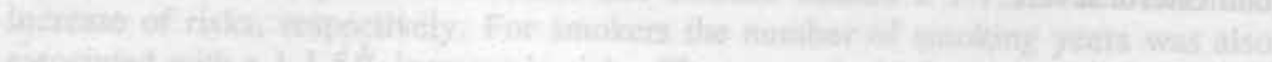

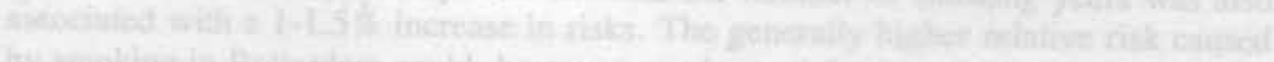

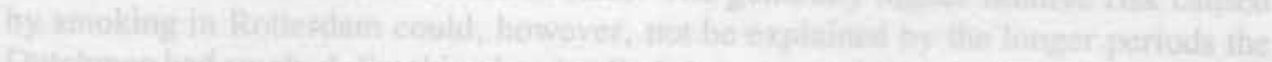

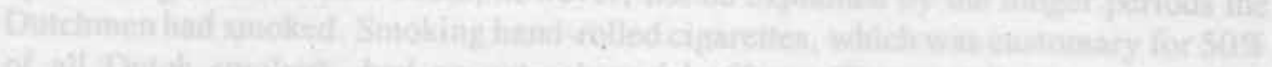

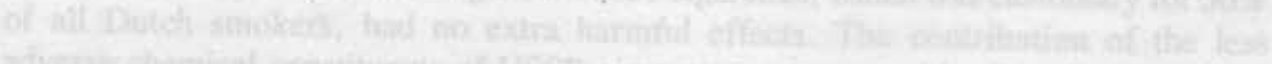

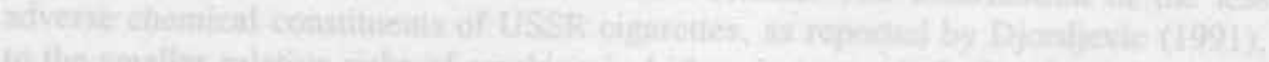

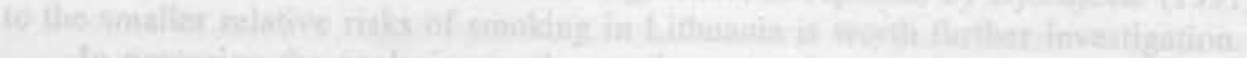

In

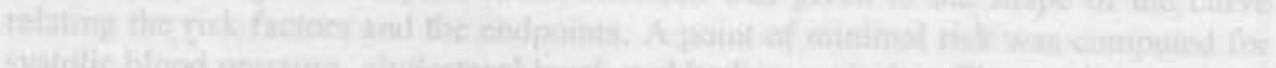

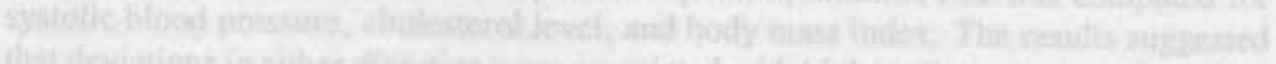

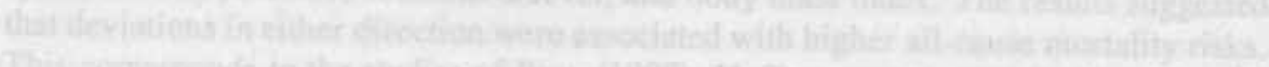

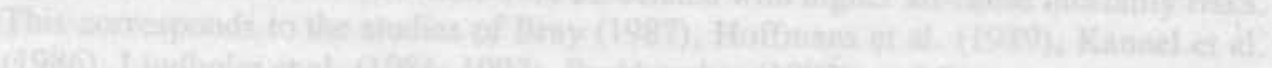

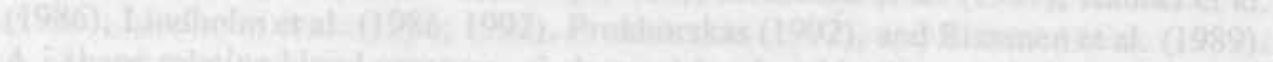

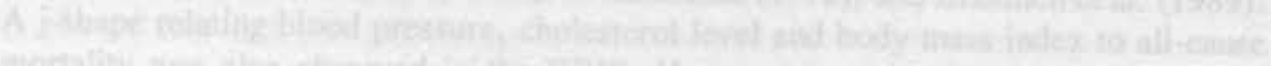

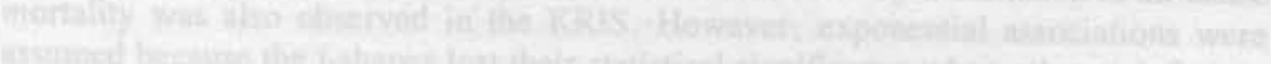

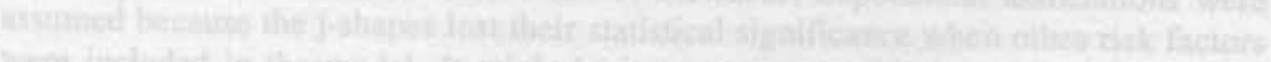

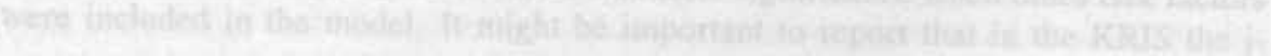


Chapter 7

\section{IMPACT OF PSYCHOSOCIAL RISK INDICATORS}

\subsection{Socioeconomic Status}

The educational level of the participants and their relatives was investigated first. Information about their wives' occupational level was poor. First, many data were missing. In Rotterdam 514 husbands (17.5\%) did not answer this question; these women had probably done household work for the largest part of their lives. In Kaunas there was a separate category for housewives $(11.2 \%)$ who had always done household work. Second, the men were asked their relatives' last occupation, which could have been a short-term job that had ended 20 or more years previously. Obviously, this indicator of SES was rather unreliable.

We began the analysis by examining the possible impact of the educational level of the participant, his wife, his parents, and his parents-in-law. A similar analysis was done for the occupational level of the participant, his father, and his father-in-law. To distinguish direct from indirect effects, these elements were simultaneously adjusted. By testing for the statistical significance of the interaction terms, several measures of status inconsistency and intergenerational mobility were studied for the impact on an individual's risk of mortality and morbidity. The two "high" categories were sometimes put together, as some analyses had to be restricted to combined variables because small numbers hindered detailed analysis. Categories of the educational level of the parents and the parents-in-law were similarly combined in each analysis.

\section{The Educational Level of the Participant}

Table 7.1 shows the age-adjusted relative risks on all four endpoints. The educational level appeared not to be significantly associated with any of the endpoints in Kaunas, although the relative risks show that the all-cause and CVD mortality were most elevated in the lowest educational groups. No particular effect on CHD mortality and nonfatal MI was found in Kaunas. Rotterdammers with a low educational level had a significantly elevated risk of all-cause mortality $(R R=1.57)$. Smaller and nonsignificant effects were found for the three cardiovascular endpoints. The relative risks were relatively small. 
Table 7.1 Age-adjusted association of the participant's educational level with endpoints (all participants).

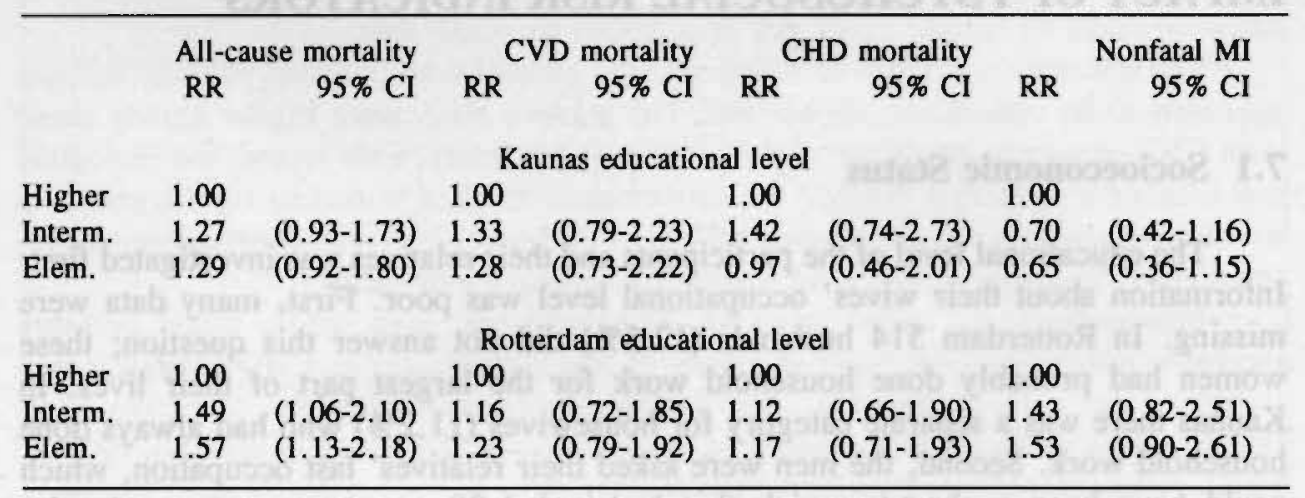

\section{The Educational Level of the Relatives}

The analysis described in this section was restricted to married participants. The numbers were 2292 in Kaunas and 2944 in Rotterdam. Table 7.2 shows that the educational level of the wife was significantly associated with all-cause mortality in both cohorts. The risk of mortality to men whose wives had a low level of education was increased by $62 \%$ in Kaunas and by $134 \%$ in Rotterdam. Except for nonfatal MI in Kaunas, risks due to the three cardiovascular endpoints were also elevated in both cohorts, although less pronounced compared to all-cause mortality.

The elevated relative risks of all-cause mortality for men whose wives had a low educational level dropped to $57 \%$ in Kaunas and to $115 \%$ in Rotterdam when they were adjusted for the educational level of the participants themselves (Table 7.3). Other relative risks for the wives' educational level also decreased somewhat. The effect of the educational level of the men themselves, however, practically disappeared, when their wives' educational level was taken into account.

Differences regarding biological and lifestyle risk factors among men whose wives had a different educational level are shown in Table 7.4. Adjusting for the men's educational level, continuous risk factors were analyzed by multiple classification analysis (F-test) and ordinal or nominal risk factors by Analysis of Tables (ANOTA) (Keller \& Verbeek, 1984). Independent of their own educational level, men whose wives had a low educational level were usually older and were more often smokers in both Lithuania and The Netherlands. Furthermore, the mean cholesterol level and the body mass index were lower for these men in Kaunas, whereas in Rotterdam they were more often physically active and fewer drank alcohol. 
Table 7.2 Age-adjusted association of the educational level of both spouses with endpoints (married participants only).

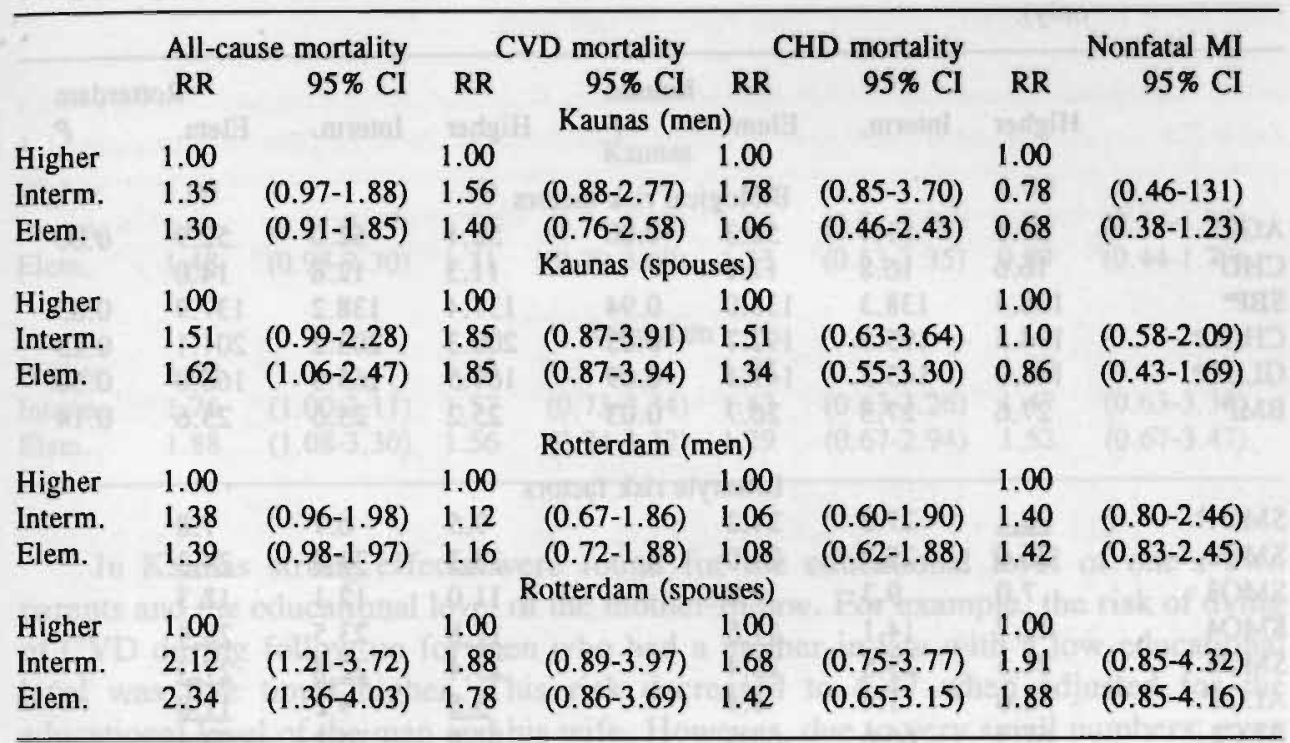

Table 7.3 Age-adjusted association of the Educational level of both Spouses with endpoints, Simultaneously Adjusted (married participants only).

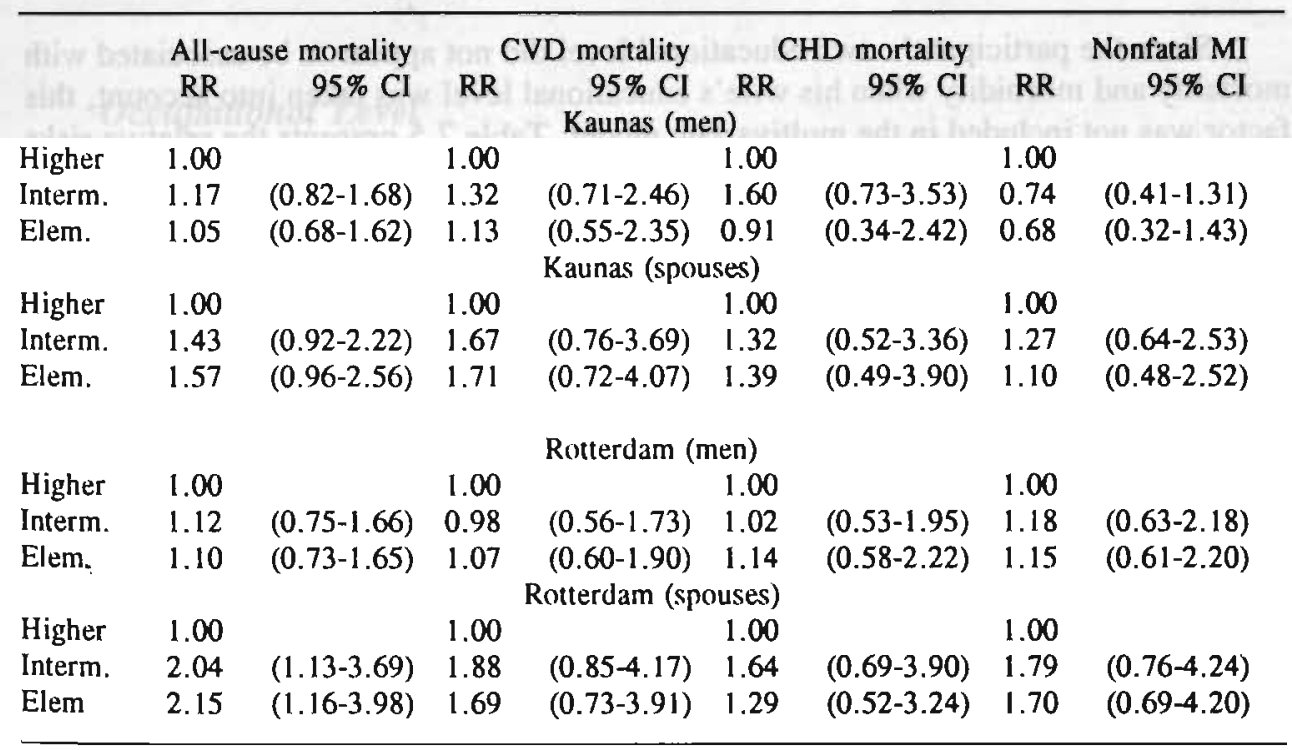


Table 7.4 Distributions of biological and lifestyle risk factors for separate categories of wives' educational level, adjusted for their husbands' educational level (married participants only).

Higher Interm. Elem. $\quad \begin{array}{r}\text { Kaunas } \\ \boldsymbol{P}\end{array}$ Higher Interm. Elem. $P$

\begin{tabular}{|c|c|c|c|c|c|c|c|c|}
\hline \multirow{2}{*}{$\mathrm{AGF}^{-1}$} & 850 & & \multicolumn{3}{|c|}{ Biological risk factors } & $24,1-57$ & Qt) & $\sin$ \\
\hline & 51.1 & 51.4 & 52.3 & 0.00 & 52.1 & 52.0 & 52.9 & 0.00 \\
\hline $\mathrm{CHD}^{b}$ & 16.6 & 16.8 & 17.1 & 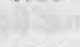 & 11.3 & 12.8 & 14.0 & \\
\hline SBP & 138.4 & 138.3 & 138.0 & 0.94 & 137.4 & 138.2 & 137.9 & 0.83 \\
\hline $\mathrm{CHOL}^{2}$ & 194.5 & 195.5 & 192.7 & 0.03 & 200.3 & 203.2 & 201.1 & 0.23 \\
\hline GLUC $^{a}$ & 143.9 & 143.3 & 149.1 & 0.09 & 161.5 & 165.0 & 166.0 & 0.50 \\
\hline $\mathrm{BMI}^{2}$ & 27.6 & 27.3 & 26.7 & 0.03 & 25.2 & 25.6 & 25.6 & 0.14 \\
\hline & & & Lifes & IBSK & 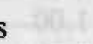 & & & \\
\hline $\mathrm{SMO1}^{\mathrm{b}}$ & 32.1 & 27.2 & 24.2 & & 9.5 & 6.7 & 7.8 & \\
\hline $\mathrm{SMO} 2$ & 26.6 & 25.7 & 24.0 & & $\underline{38.3}$ & $\underline{34.8}$ & $\underline{24.3}$ & \\
\hline SMO3 & 7.0 & 9.3 & 7.8 & & $\overline{11.0}$ & $\overline{12.1}$ & 12.3 & \\
\hline SMO4 & 12.7 & 14.1 & 17.5 & & 17.8 & 23.5 & $\underline{28.4}$ & \\
\hline SMOS & 21.5 & 23.5 & $\overline{26.4}$ & 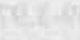 & 23.4 & 22.8 & $\overline{27.3}$ & \\
\hline $\mathrm{ALCl}^{\mathrm{b}}$ & 2.6 & 1.4 & 1.4 & 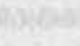 & 5.5 & $\overline{9.2}$ & 11.8 & \\
\hline ALC2 & 67.3 & 66.6 & 68.5 & & $\underline{81.8}$ & 76.2 & $\underline{73.4}$ & \\
\hline ALC3 & 30.1 & 32.0 & 30.1 & & 12.6 & 14.6 & 14.8 & \\
\hline $\mathrm{PHA}^{\mathbf{a}}$ & 208.2 & 207.3 & 208.8 & 0.91 & 142.3 & 150.8 & 153.8 & 0.00 \\
\hline
\end{tabular}

" mean (F-test), ${ }^{b}$ underlined percentage: $P<=0.05$

Since the participant's own educational level did not appear to be associated with mortality and morbidity when his wife's educational level was taken into account, this factor was not included in the multivariate model. Table 7.5 presents the relative risks of the educational level of the wives, adjusted for all biological and lifestyle risk factors. This table shows that the relative risks decreased when all the risk factors were taken into account. This was primarily caused by the higher prevalence of smoking among men whose wives had a low educational level. In Rotterdam, relative risks of all-cause mortality remained significantly elevated for men whose wives had an intermediate or low educational level ( $R R=1.76$ and 1.88$)$.

These findings indicate that a spouse has a strong influence on the life expectancy of her husband. This influence is only partly due to established biological and lifestyle risk factors. The data strongly suggest that yet unknown cultural factors associated with a wife's educational level influence the health of her husband.

The influence of the educational level of the parents and the parents-in-law on the morbidity and mortality was analyzed. The result shows that in Rotterdam the educational level of the father-in-law affected a man's risk of all-cause mortality (RR $=1.90$ ) significantly. This relative risk decreased to 1.28 when adjusted for the educational level of the man and his wife. 
Table 7.5

Association of the educational level of wives with endpoints, adjusted for all biological and lifestyle risk factors (married participants only).

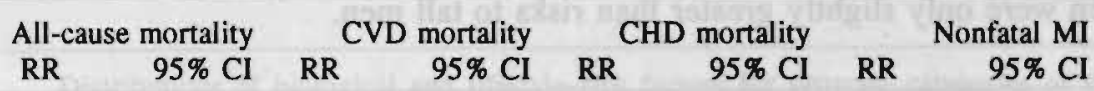

\begin{tabular}{lllllllll} 
& \multicolumn{7}{c}{ Kaunas } \\
Higher & 1.00 & & 1.00 & & 1.00 & & 1.00 & \\
Interm. & 1.41 & $(0.91-2.17)$ & 1.58 & $(0.74-3.38)$ & 1.25 & $(0.51-3.06)$ & 1.04 & $(0.54-1.99)$ \\
Elem. & 1.48 & $(0.95-2.30)$ & 1.71 & $(0.79-3.69)$ & 1.33 & $(0.53-3.35)$ & 0.89 & $(0.44-1.79)$
\end{tabular}

\begin{tabular}{llllllllll} 
& & \multicolumn{7}{c}{ Rotterdam } \\
Higher & 1.00 & & 1.00 & & 1.00 & & 1.00 & \\
Interm. & 1.76 & $(1.00-3.11)$ & 1.57 & $(0.73-3.34)$ & 1.43 & $(0.63-3.26)$ & 1.47 & $(0.63-3.38)$ \\
Elem. & 1.88 & $(1.08-3.30)$ & 1.56 & $(0.74-3.32)$ & 1.29 & $(0.67-2.94)$ & 1.52 & $(0.67-3.47)$
\end{tabular}

In Kaunas strong effects were found for the educational level of one's own parents and the educational level of the mother-in-law. For example, the risk of dying of CVD during follow-up for men who had a mother-in-law with a low educational level was five times higher. This risk decreased to 4.47 when adjusted for the educational level of the man and his wife. However, due to very small numbers, even this strong relative risk was imprecise and far from significant. Therefore, the data do not prove that the educational level of one's parents or parents-in-law influence longevity. They only suggest that further explorations of the association of family traditions with health may be worthwhile.

\section{Occupational Level}

Table 7.6 shows the associations of the men's occupational level with their risk of future mortality and morbidity. Low occupational SES was significantly associated with mortality in both cohorts. Kaunas workers had a $30 \%$ higher all-cause mortality risk than employees. Risks in Rotterdam were strongly elevated for those with a low or middle occupational level. Risks among these groups were approximately twice as high as those of men with a high occupational level. As with the educational level, it is important to note that the lower SES groups in Kaunas tended to have a lower risk of nonfatal MI, while the opposite trend was observed in Rotterdam.

When educational and occupational levels were simultaneously adjusted, the effeet of the educational level practically disappeared for all endpoints. This is shown in Table 7.7 for all-cause mortality. The effect of the occupational level also decreased, although not as much as the effect of the educational level.

Background SES indicators (educational and occupational levels of parents) also did not explain the association of a person's occupational level with his risk of mortality, nor did body height. Height was taken into account, as to some extent it 
reflects material conditions and nutritional factors during upbringing or diseases experienced in childhood (Floud, 1989; Nyström Peck, 1992; Stronks et al., 1993; West, 1991). Lower occupational classes were somewhat shorter, but risks to short men were only slightly greater than risks to tall men.

Table 7.6 Age-adjusted association of the occupational level with endpoints (all participants).

\begin{tabular}{|c|c|c|c|c|c|c|c|c|}
\hline & \multicolumn{2}{|c|}{ All-cause mortality } & \multicolumn{2}{|c|}{ CVD mortality } & \multicolumn{2}{|c|}{ CHD mortality } & \multicolumn{2}{|r|}{ Nonfatal MI } \\
\hline & $\mathbf{R R}$ & $95 \% \mathrm{CI}$ & RR & $95 \% \mathrm{CI}$ & RR & $95 \% \mathrm{CI}$ & RR & $95 \% \mathrm{CI}$ \\
\hline sibi: & & 74 & & Kaunas & & nis & & 0.68 \\
\hline Employee & 1.00 & & 1.00 & & 1.00 & & 1.00 & \\
\hline Worker & 1.30 & $(1.03-1.65)$ & 1.20 & $(0.81-1.77)$ & 1.20 & $(0.73-1.97)$ & 0.86 & $(0.56-1.33)$ \\
\hline (ch & (0) & ste & (6) & Rotterdam & & ait? & in 19 & 68.1 \\
\hline Higher & 1.00 & & 1.00 & & 1.00 & & 1.00 & \\
\hline Employee & 1.84 & $(1.22-2.79)$ & 2.14 & $(1.16-3.95)$ & 1.93 & $(0.98-3.81)$ & 1.31 & $(0.72-2.40)$ \\
\hline Worker & 2.01 & $(1.34-3.01)$ & 1.98 & $(0.97-3.64)$ & 1.89 & $(0.97-3.68)$ & 1.41 & $(0.78-2.55)$ \\
\hline
\end{tabular}

Table 7.7 Association of the educational and occupational level with all-cause mortality, adjusted for age and each other (all participants).

\begin{tabular}{lcccccccc}
\hline & RR & $\begin{array}{r}95 \% \mathrm{CI} \\
\text { Kaunas }\end{array}$ & $\begin{array}{r}\text { RR } \\
\text { Education }\end{array}$ & Occupation & & RR & $\begin{array}{r}95 \% \mathrm{CI} \\
\text { Rotterdam } \\
\text { Education }\end{array}$ & $\begin{array}{r}\text { RR } \\
\text { Occupation }\end{array}$ \\
High & 1.00 & & & & 1.00 & & 1.00 & \\
Middle & 1.10 & $(0.77-1.58)$ & 1.00 & & 1.13 & $(0.75-1.69)$ & 1.71 & $(1.06-2.75)$ \\
Low & 1.03 & $(0.67-1.58)$ & 1.29 & $(0.95-1.75)$ & 1.11 & $(0.73-1.69)$ & 1.85 & $(1.11-3.20)$ \\
\hline
\end{tabular}

Table 7.8 presents the distribution of the risk factors in the different occupational groups. The table shows that smoking was more prevalent among the lowest occupational groups in both cohorts. The body mass index was higher among the higher social classes in Lithuania and The Netherlands, as was the cholesterol level in Kaunas. A similar trend was observed in Rotterdam. Physical activity tended to be higher among workers in both Lithuania and The Netherlands. CHD-at-screening was more prevalent in the lower Dutch occupational groups. No difference in CHD prevalence was observed in Kaunas. Immoderate alcohol consumption was more prevalent among workers in Kaunas. In Rotterdam the highest occupational group was characterized by moderate alcohol consumption, while the lowest occupational group was characterized either by no drinking or by heavy drinking. No differences in systolic blood pressure and glucose tolerance were observed between the occupational groups. Hence, as far as risk factors are concerned the KRIS data gave mixed 
evidence for the association of low SES with coronary risk factors. Smoking was more prevalent among the lower strata, while the mean cholesterol level and body mass index were higher in the upper strata.

Table 7.8 Distributions of biological and lifestyle risk factors for separate categories of the occupational level (all participants).

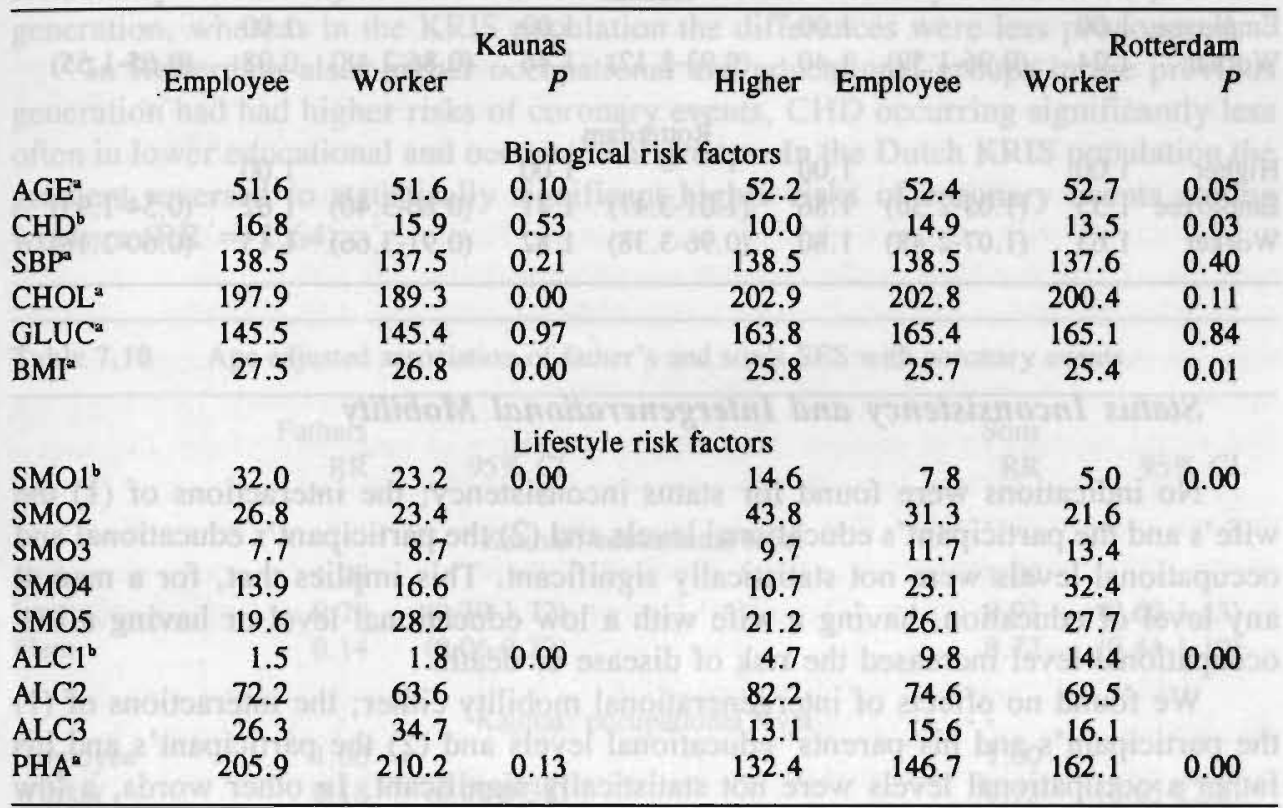

${ }^{a}$ mean (F-test), ${ }^{b}$ percentage (chi ${ }^{2}$ test)

Table 7.9 shows that the relative risks of all-cause mortality in Rotterdam decreased after adjustment for the biological and lifestyle risk factors, while they increased in Kaunas for the cardiovascular endpoints. The decrease in Rotterdam was caused by a higher CHD and smoking prevalence among the lower SES groups. The mortality risks for workers and middle level employees were more than $50 \%$ higher than those of men of higher occupations. Especially because of the relatively low cholesterol levels among the Kaunas lower socioeconomic groups, risks of mortality caused by CVD and CHD increased. Nonfatal MI was not associated with SES. Hence, the general picture shows that in both cohorts the social class gradient relating SES to all-cause mortality and cause-specific mortality was only partially based upon biological and lifestyle risk factors. 


\begin{tabular}{|c|c|c|c|c|c|c|c|c|}
\hline \multirow{3}{*}{ Table 7.9} & \multicolumn{8}{|c|}{$\begin{array}{l}\text { Association of the occupational level with endpoints, adjusted for all biological and } \\
\text { lifestyle risk factors (all participants). }\end{array}$} \\
\hline & \multicolumn{2}{|c|}{ All-cause mortality } & \multicolumn{2}{|c|}{ CVD mortality } & \multicolumn{2}{|c|}{ CHD mortality } & \multicolumn{2}{|r|}{ Nonfatal MI } \\
\hline & RR & $95 \% \mathrm{CI}$ & RR & $95 \% \mathrm{CI}$ & RR & $95 \% \mathrm{CI}$ & RR & $95 \% \mathrm{Cl}$ \\
\hline Densto 7.6 & $x$ & 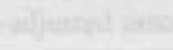 & 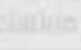 & Kaunas & & & & F \\
\hline \multirow[t]{2}{*}{ Worker } & $\begin{array}{l}1.00 \\
1.24\end{array}$ & $(0.96-1.59)$ & $\begin{array}{l}1.00 \\
1.40\end{array}$ & $(0.93-2.12)$ & $\begin{array}{l}1.00 \\
1.46\end{array}$ & $(0.86-2.48)$ & $\begin{array}{l}1.00 \\
0.98\end{array}$ & $(0.65-1.55)$ \\
\hline & & & & Rotterdam & & & & \\
\hline Higher & 1.00 & & 1.00 & & 1.00 & & 1.00 & \\
\hline Employee & 1.55 & $(1.02-2.36)$ & 1.86 & $(1.01-3.47)$ & 1.71 & $(0.86-3.40)$ & 1.02 & $(0.54-1.93)$ \\
\hline Worker & 1.63 & $(1.07-2.48)$ & 1.80 & $(0.96-3.38)$ & 1.82 & $(0.91-3.66)$ & 1.15 & $(0.60-2.18)$ \\
\hline
\end{tabular}

\section{Status Inconsistency and Intergenerational Mobility}

No indications were found for status inconsistency; the interactions of (1) the wife's and the participant's educational levels and (2) the participant's educational and occupational levels were not statistically significant. This implies that, for a man at any level of education, having a wife with a low educational level or having a low occupational level increased the risk of disease or death.

We found no effects of intergenerational mobility either; the interactions of (1) the participant's and his parents' educational levels and (2) the participant's and his father's occupational levels were not statistically significant. In other words, a low SES not only had detrimental health consequences in the case that the father had a high SES, but also in case that the father had a low SES.

The absence of any particular effects of incongruence suggests that effects of particular SES indicators are homogeneously associated with mortality and morbidity across categories of other SES indicators.

\section{The Changing Gradient}

Table 7.10 presents the age-adjusted odds ratios of coronary events for the participant's and his father's SES. Because the only information available about the cardiovascular health of the parents was the answer to the question "Has he or she ever suffered from MI?" it was not known whether a positive answer suggested a fatal or a nonfatal MI. To compare like with like, the analysis of the association of the participant's SES with cardiovascular health was repeated with CHD mortality and nonfatal MI combined into one category of "coronary events". The analysis for the fathers was adjusted for the fathers' ages at death or at the screening (interaction term) and the analysis for the sons was adjusted for their ages. 
As this table shows, in the previous generation coronary events occurred more often in the higher strata, resulting in statistically significant lower probabilities of coronary events in lower occupational groups. Especially in Kaunas, CHD was much less common in lower occupational groups, such as farmers $(R R=0.15)$ and workers $(\mathrm{RR}=0.18)$. Occupational level was not related to coronary events among the sons in Kaunas. Similar results were found for educational level where those with elementary school only had had a much smaller risk of coronary events in the previous generation, whereas in the KRIS population the differences were less pronounced.

In Rotterdam also, higher occupational and educational groups in the previous generation had had higher risks of coronary events, CHD occurring significantly less often in lower educational and occupational groups. In the Dutch KRIS population the gradient reversed to statistically significant higher risks of coronary events among workers $(R R=1.64)$.

Table 7.10 Age-adjusted association of father's and son's SES with coronary events.

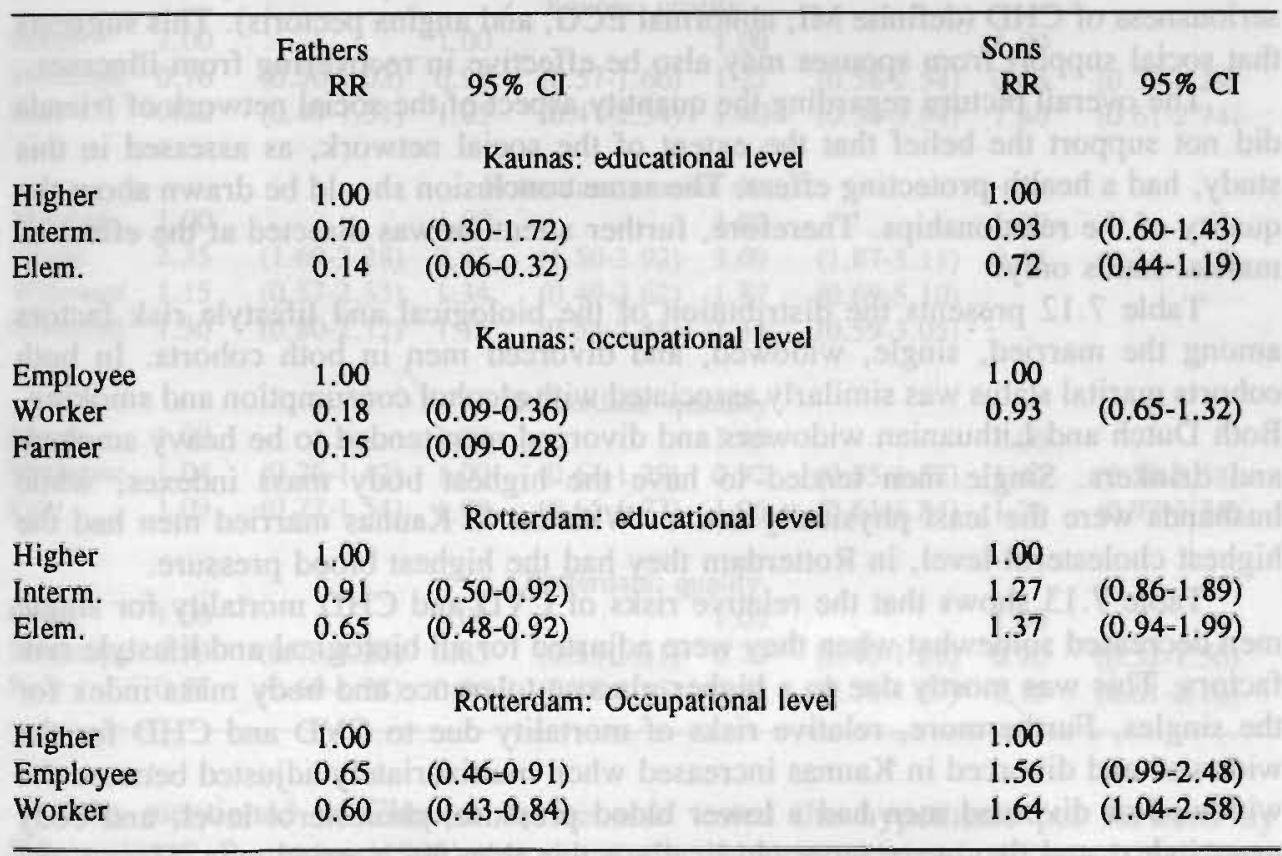

\subsection{Social Network}

As mentioned in Chapter 4, three indicators of social network were used: quantity and quality of social relationships and marital status. Age-adjusted relative risks are presented in Table 7.11. Since in both cohorts none of the widowers suffered 
a nonfatal MI, all nonmarried men were compared to the married men for nonfatal MI. As shown in this table, all-cause mortality risks of all nonmarried groups were higher than those of the married groups. The highest all-cause mortality risks in Kaunas were found among the widowed $(R R=2.14)$. Divorced men in Lithuania had increased risks of mortality caused by CVD and CHD. Being nonmarried appeared not to affect the risk of nonfatal MI. Dutch single men had elevated mortality risks due to all causes $(R R=2.35), C V D(R R=2.42)$, and CHD $(R R=3.09)$. The widowers in Rotterdam tended to have an increased risk of CHD mortality. However, the association was not statistically significant. Unfortunately, we could not determine the recency of widowhood; when the spouses died is unknown to us.

No association was found between marital status and nonfatal MI (both among those with and without CHD-at-screening). It was also found that marital status was not associated with presence of CHD-at-screening. This may suggest that nonmarried men are at risk for fatal cardiovascular events especially.

When the effect of marital status on mortality among men with CHD-at-screening was investigated, the effects were large, even when they were adjusted for the seriousness of CHD (definite MI, abnormal ECG, and angina pectoris). This suggests that social support from spouses may also be effective in recovering from illnesses.

The overall picture regarding the quantity aspect of the social network of friends did not support the belief that the extent of the social network, as assessed in this study, had a health-protecting effect. The same conclusion should be drawn about the quality of the relationships. Therefore, further attention was directed at the effect of marital status only.

Table 7.12 presents the distribution of the biological and lifestyle risk factors among the married, single, widowed, and divorced men in both cohorts. In both cohorts marital status was similarly associated with alcohol consumption and smoking. Both Dutch and Lithuanian widowers and divorced men tended to be heavy smokers and drinkers. Single men tended to have the highest body mass indexes, while husbands were the least physically active. Whereas in Kaunas married men had the highest cholesterol level, in Rotterdam they had the highest blood pressure.

Table 7.13 shows that the relative risks of CVD and CHD mortality for single men decreased somewhat when they were adjusted for all biological and lifestyle risk factors. This was mostly due to a higher glucose tolerance and body mass index for the singles. Furthermore, relative risks of mortality due to CVD and CHD for the widowed and divorced in Kaunas increased when multivariately adjusted because the widowed or divorced men had a lower blood pressure, cholesterol level, and body mass index, and they were more physically active than the married men. Hence, the main conclusion is that the difference in mortality risks between married and nonmarried men, as observed in both cohorts, cannot be explained by the traditional biological and lifestyle risk factors. 
Table 7.11 Age-adjusted association of marital status and quantity and quality of social relationships with endpoints.

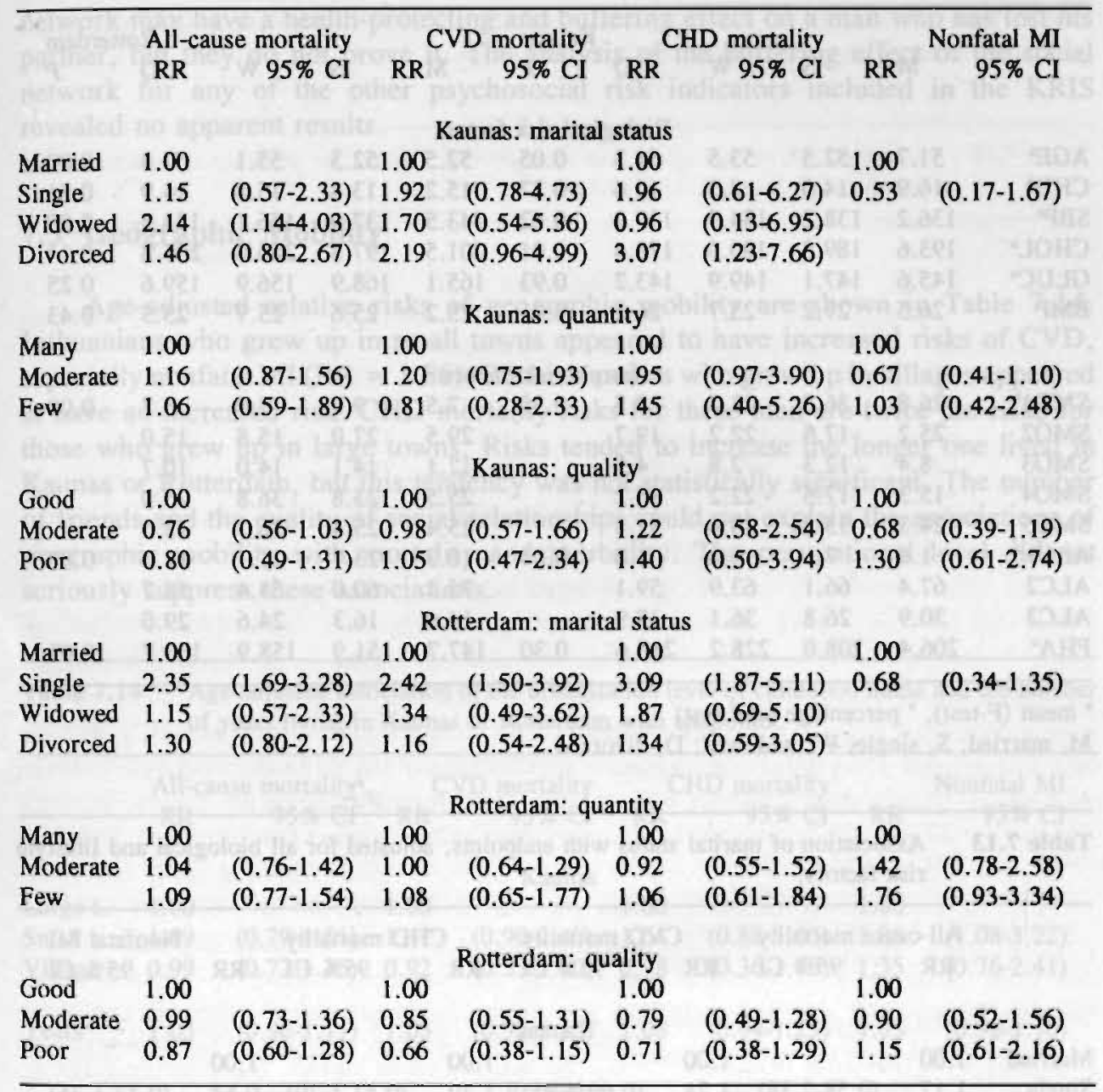

As mentioned in Chapter 4, we also tested the hypothesis put forward by Silverstein \& Bengtson (1991) that the stress of being widowed, divorced, or single can be partially offset by affectionate relationships with children or friends. To investigate this buffering effect of the quantity and quality of the social network among nonmarried men, the cohorts were combined, and the divorced and widowed men put into one category to avoid the problem of empty cells. The results show that the hypothesis of Silverstein \& Bengtson is attractive. 
Table 7.12 Distributions of biological and lifestyle risk factors for separate categories of marital status.

$$
\begin{array}{lllllllll}
\text { M } & \text { S } & \text { W } & \text { D } & P & \text { M } & \text { S } & \text { W } & \text { D }
\end{array}
$$

Biological risk factors

$\begin{array}{lrrrrrrrrrr}\text { AGE }^{a} & 51.7 & 52.5 & 53.5 & 51.3 & 0.05 & 52.5 & 52.3 & 55.1 & 52.4 & 0.00 \\ \text { CHD }^{b} & 16.9 & 14.0 & 8.3 & 10.6 & 0.27 & 15.2 & 13.4 & 15.8 & 14.9 & 0.81 \\ \text { SBP }^{a} & 136.2 & 138.2 & 134.9 & 137.2 & 0.62 & 143.5 & 137.9 & 136.1 & 134.5 & 0.00 \\ \text { CHOL }^{2} & 193.6 & 189.8 & 190.0 & 178.4 & 0.01 & 201.5 & 197.8 & 206.0 & 205.8 & 0.14 \\ \text { GLUC }^{a} & 145.6 & 147.1 & 149.9 & 143.2 & 0.93 & 165.1 & 168.9 & 156.9 & 159.6 & 0.25 \\ \text { BMI }^{2} & 26.5 & 27.2 & 25.7 & 26.2 & 0.01 & 25.2 & 25.6 & 25.7 & 25.5 & 0.43\end{array}$

\begin{tabular}{|c|c|c|c|c|c|c|c|c|c|c|}
\hline $\mathrm{SMO}^{\mathrm{b}}$ & 26.8 & 36.8 & 22.2 & 28.8 & 0.35 & 7.5 & 9.7 & 7.0 & 5.7 & 0.00 \\
\hline SMO2 & 25.2 & 17.5 & 22.2 & 19.7 & & 29.5 & 27.0 & 15.8 & 15.0 & \\
\hline SMO3 & 8.4 & 12.3 & 2.8 & 4.5 & & 12.1 & 14.1 & 14.0 & 10.7 & \\
\hline SMO4 & 15.3 & 17.5 & 22.2 & 15.2 & & 25.5 & 23.8 & 36.8 & 25.0 & \\
\hline SMO5 & 24.3 & 15.8 & 30.6 & 31.8 & & 25.4 & 25.4 & 26.3 & 43.6 & \\
\hline $\mathrm{ALCl}^{\mathrm{b}}$ & 1.6 & 7.1 & 0.0 & 3.0 & 0.04 & 10.3 & 23.7 & 21.1 & 12.3 & 0.00 \\
\hline ALC2 & 67.4 & 66.1 & 63.9 & 59.1 & to & 75.1 & 60.0 & 54.4 & 58.7 & \\
\hline ALC3 & 30.9 & 26.8 & 36.1 & 37.9 & & 14.6 & 16.3 & 24.6 & 29.0 & \\
\hline $\mathrm{PHA}^{2}$ & 206.4 & 208.0 & 228.2 & 215.6 & 0.30 & 147.7 & 151.9 & 158.9 & 160.7 & 0.02 \\
\hline
\end{tabular}

a mean (F-test), ${ }^{b}$ percentage (chi ${ }^{2}$ test)

$M$, married; S, single; W, widowed; $D$, divorced

Table 7.13 Association of marital status with endpoints, adjusted for all biological and lifestyle risk factors.

\begin{tabular}{|c|c|c|c|c|c|c|c|c|}
\hline & \multicolumn{2}{|c|}{ All-cause mortality } & \multicolumn{2}{|c|}{ CVD mortality } & \multicolumn{2}{|c|}{ CHD mortality } & \multicolumn{2}{|r|}{ Nonfatal MI } \\
\hline & $\mathrm{RR}$ & $95 \% \mathrm{Cl}$ & RR & $95 \% \mathrm{CI}$ & RR & $95 \%$ CI & RR & $95 \% \mathrm{CI}$ \\
\hline & & & & Kaunas & & & & \\
\hline Married & 1.00 & & 1.00 & & 1.00 & & 1.00 & \\
\hline Single & 1.17 & $(0.58-2.38)$ & 1.74 & $(0.69-4.41)$ & 1.39 & $(0.41-4.79)$ & 0.54 & $(0.17-1.75)$ \\
\hline Widowed & 2.17 & $(1.15-4.10)$ & 2.16 & $(0.68-6.91)$ & 1.06 & $(0.14-7.75)$ & & \\
\hline Divorced & 1.55 & $(0.84-2.86)$ & 2.97 & $(1.28-6.88)$ & 4.92 & $(1.93-12.5)$ & & \\
\hline & & & & Rotterdan & & & & \\
\hline Married & 1.00 & & 1.00 & & 1.00 & & 1.00 & \\
\hline Single & 2.12 & $(1.49-3.01)$ & 2.19 & $(1.32-3.63)$ & 3.06 & $(1.80-5.20)$ & 0.65 & $(0.32-1.31)$ \\
\hline Widowed & 1.16 & $(0.57-2.35)$ & 1.44 & $(0.53-3.92)$ & 1.93 & $(0.71-5.29)$ & & \\
\hline Divorced & 1.24 & $(0.76-2.04)$ & 1.11 & $(0.51-2.40)$ & 1.27 & $(0.55-2.93)$ & & \\
\hline
\end{tabular}

The relative risk for CVD mortality among divorced and widowed men with a limited number of friends was 1.95 (95\% CI: $0.46,8.37$ ) and for CHD mortality 5.18 
(95\% CI: $0.59,45.71$ ) compared to divorced and widowed men with many friends. Due to small numbers, these relative risks were not statistically significant. Therefore, we must conclude that the data give some support to the belief that an extended network may have a health-protecting and buffering effect on a man who has lost his partner, but they do not prove it. The analysis of the buffering effect of the social network for any of the other psychosocial risk indicators included in the KRIS revealed no apparent results.

\subsection{Geographic Mobility}

Age-adjusted relative risks of geographic mobility are shown in Table 7.14. Lithuanians who grew up in small towns appeared to have increased risks of CVD, especially nonfatal MI $(R R=1.86)$. Rotterdammers who grew up in villages appeared to have an increased risk. CHD mortality risks for these men are twice the risks for those who grew up in large towns. Risks tended to increase the longer one lived in Kaunas or Rotterdam, but this tendency was not statistically significant. The number of friends and the quality of social relationships could not explain the associations of geographic mobility with mortality and morbidity. The occupational level did not seriously suppress these associations.

Table 7.14 Age-adjusted association of the urbanization level of childhood home and the number of years living in Kaunas or Rotterdam with endpoints.

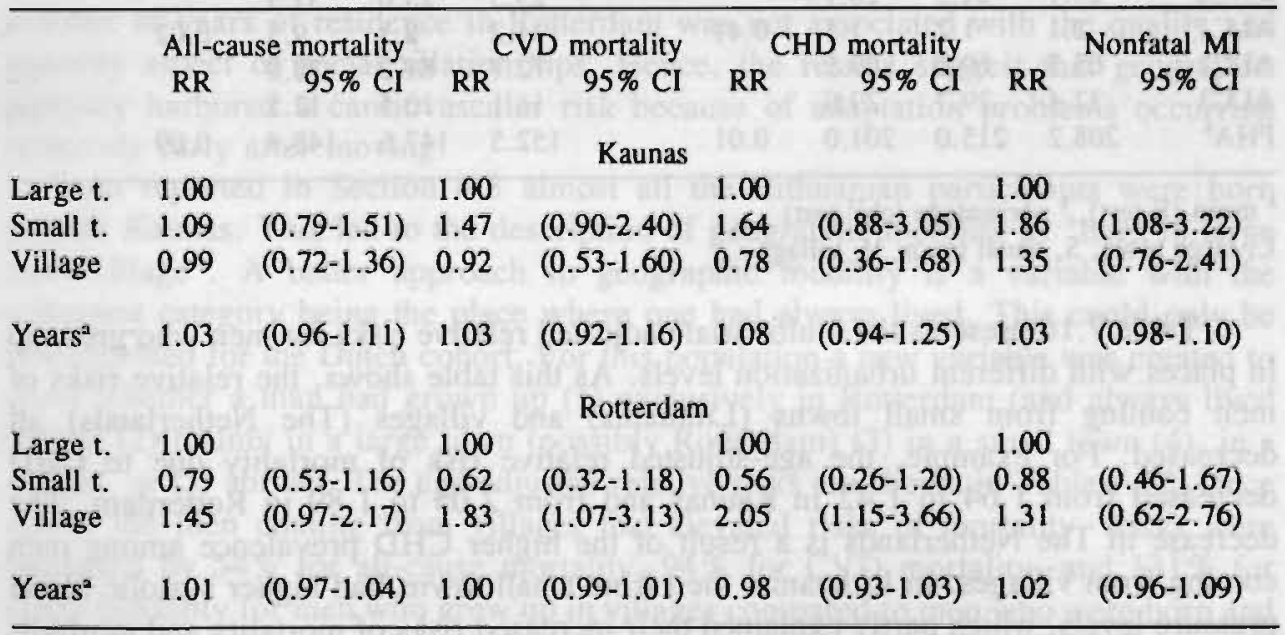

aRelative risks show the increase of the risks for five-year periods.

Table 7.15 shows that men who grew up in small towns in Lithuania had the highest systolic blood pressure (140 $\mathrm{mmHg}$ ). Together with the men who came from villages, they also had higher cholesterol levels than men who grew up in large cities. 
The Lithuanians from villages appeared to be the least physically active. The Dutchmen who grew up in large towns had a higher mean physical activity than men from small towns or villages. Heavy smoking tended to be more common among Dutch men coming from villages, and immoderate alcohol consumption was more prevalent among men who grew up in a large city.

Table 7.15 Distributions of biological and lifestyle risk factors for separate categories of the urbanization level of childhood home.

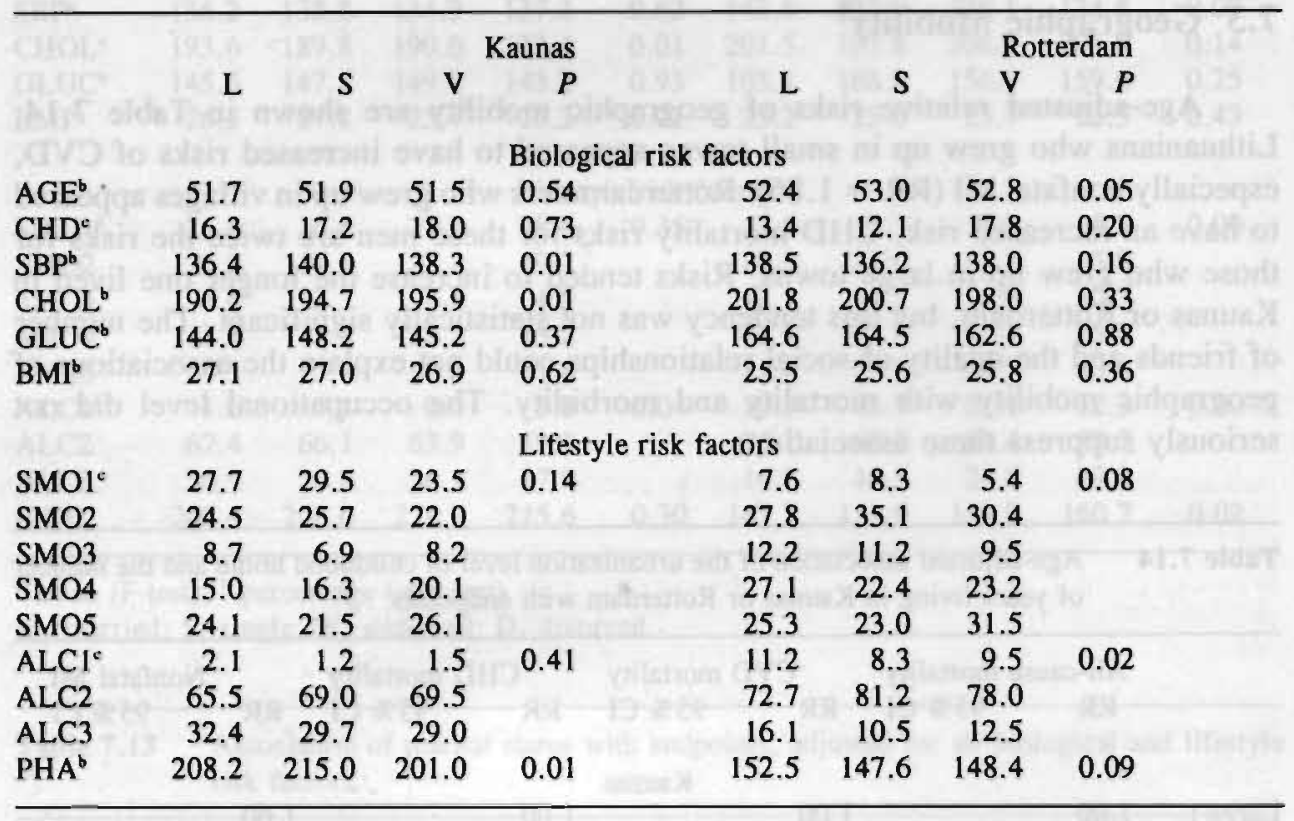

mean (F-test), ${ }^{b}$ percentage (chi ${ }^{2}$ test)

L. large town; $S$, small town; V, village

Table 7.16 presents the multivariate adjusted relative risks for men who grew up in places with different urbanization levels. As this table shows, the relative risks of men coming from small towns (Lithuania) and villages (The Netherlands) all decreased. For example, the age-adjusted relative risk of mortality due to CHD decreased from 1.64 to 1.42 in Kaunas and from 2.05 to 1.89 in Rotterdam. The decrease in The Netherlands is a result of the higher CHD prevalence among men coming from villages. In Lithuania, men from small towns had higher systolic blood pressure levels, which partly explained their increased risks of mortality and morbidity. Hence, it appears that geographic mobility exerted its influence on mortality partially through increased blood pressure or factors leading to CHD at an early age. 
Table 7.16 Association of the urbanization level of childhood home with endpoints, adjusted for all biological and lifestyle risk factors.

\begin{tabular}{|c|c|c|c|c|c|c|c|c|}
\hline \multirow{2}{*}{$40+5 \times 10$} & \multicolumn{2}{|c|}{ All-cause mortality } & \multicolumn{2}{|c|}{ CVD mortality } & \multicolumn{2}{|c|}{ CHD mortality } & \multirow[b]{2}{*}{ RR } & \multirow{2}{*}{$\begin{array}{r}\text { Nonfatal MI } \\
95 \% \mathrm{CI}\end{array}$} \\
\hline & RR & $95 \% \mathrm{Cl}$ & RR & $95 \% \mathrm{CI}$ & RR & $95 \% \mathrm{CI}$ & & \\
\hline & & & & Kaunas & & & & \\
\hline Large $t$. & 1.00 & & 1.00 & & 1.00 & & 1.00 & \\
\hline Small t. & 1.05 & $(0.75-1.46)$ & 1.14 & $(0.68-1.92)$ & 1.42 & $(0.73-2.78)$ & 1.65 & $(0.92-2.96)$ \\
\hline Village & 0.84 & $(0.60-1.17)$ & 0.71 & $(0.41-1.26)$ & 0.62 & $(0.28-1.36)$ & 1.18 & $(0.65-2.13)$ \\
\hline & & & & Rotterdan & & & & \\
\hline Large $t$. & 1.00 & & 1.00 & & 1.00 & & 1.00 & \\
\hline Small $\mathrm{t}$. & 0.89 & $(0.59-1.32)$ & 0.69 & $(0.35-1.37)$ & 0.68 & $(0.32-1.48)$ & 0.92 & $(0.46-1.82)$ \\
\hline Village & 1.28 & $(0.85-1.95)$ & 1.62 & $(0.93-2.82)$ & 1.89 & $(1.04-3.44)$ & 1.29 & $(0.59-2.84)$ \\
\hline
\end{tabular}

Subsequently, it was investigated whether the risk to men who grew up in small towns in Lithuania or in villages in The Netherlands decreased as the number of years of residence in Kaunas or Rotterdam increased. The results show that the risk for CVD tended to decrease as the men lived longer in the city, especially in The Netherlands. Although nonsignificant, the results for the Dutch part of the KRIS suggest that the risks of cardiovascular events decreased by $8-10 \%$ for each 5 years of residence in Rotterdam. Detailed analysis shows that the presence of CHD-atscreening decreased the longer that the men from villages had lived in Rotterdam. The number of years of residence in Rotterdam was not associated with the quality and quantity aspect of social relationships. Hence, the results suggest that geographic mobility harbored a cardiovascular risk because of adaptation problems occurring relatively early after moving.

As reported in Section 4.3 almost all the Lithuanian participants were born outside Kaunas. This led to the description of geographic mobility as "born in large town-village". A better approach to geographic mobility is a variable with the reference category being the place where one had always lived. This could only be implemented for the Dutch cohort. For this population a new variable was created to show whether a man had grown up (1) exclusively in Rotterdam (and always lived there), (2) mainly in a large town (possibly Rotterdam) (3) in a small town (4), in a village, or (5) abroad. The age-adjusted relative risks are shown in Table 7.17. Once again, the men coming from villages had elevated risks of mortality. Risks were increased by $54 \%$ for all-cause mortality, $94 \%$ for CVD mortality, and $131 \%$ for CHD mortality for men who grew up in villages compared to men who were born and grew up in Rotterdam. As only 87 men grew up abroad, the decreased risks for these men were highly unreliable.

For those who grew up abroad, the number of years of residence in Rotterdam did not increase the risks; risks did not become more similar to men who had always lived in Rotterdam. In fact, the risks decreased even further (statistically nonsignifi- 
cant). The increased risks for men from the villages were, as before, partly based on higher CHD-prevalence rates, which decreased the longer the men lived in Rotterdam.

Table 7.17 Age-adjusted association of the urbanization level of childhood home in Rotterdam (new variable) with endpoints.

\begin{tabular}{|c|c|c|c|c|c|c|c|c|}
\hline \multirow{2}{*}{ 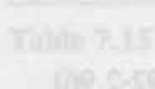 } & \multicolumn{2}{|c|}{ All-cause mortality } & \multicolumn{2}{|c|}{ CVD mortality } & \multicolumn{2}{|c|}{ CHD mortality } & \multicolumn{2}{|r|}{ Nonfatal MI } \\
\hline & RR & $95 \% \mathrm{CI}$ & RR & $95 \% \mathrm{Cl}$ & RR & $95 \% \mathrm{CI}$ & RR & $95 \% \mathrm{CI}$ \\
\hline Alw: & 1.00 & & 1.00 & & 1.00 & & 1.00 & \\
\hline Large $t$. & 1.13 & $(0.88-1.44)$ & 1.13 & $(0.79-1.61)$ & 1.30 & $(0.87-1.94)$ & 0.90 & $(0.59-1.38)$ \\
\hline Small $\mathrm{t}$. & 0.83 & $(0.55-1.24)$ & 0.65 & $(0.33-1.25)$ & 0.62 & $(0.28-1.37)$ & 0.84 & (0.44-1.63) \\
\hline Village & 1.54 & $(1.02-2.34)$ & 1.94 & $(1.11-3.39)$ & 2.31 & $(1.26-4.24)$ & 1.28 & $(0.60-2.74)$ \\
\hline Abroad & 0.69 & $(0.31-1.56)$ & 0.50 & $(0.12-2.02)$ & 0.34 & $(0.05-2.45)$ & 0.94 & $(0.29-3.09)$ \\
\hline
\end{tabular}

\subsection{Type A Behavior Pattern}

No indications were found that the type A behavior pattern, as assessed by the JAS, was associated with mortality or morbidity (Table 7.18). Although moderate type As in Kaunas had highly elevated age-adjusted risks of CVD and CHD mortality, this effect was absent for the extreme type As. This unpredicted curvilinearity makes these findings hard to interpret. In The Netherlands type A was clearly not associated with heart disease. Using the continuous score did not change the results. These results were neither different for men with or without CHD-at-screening nor were these associations suppressed by any of the biological or lifestyle risk factors, as relative risks hardly changed when they were adjusted for these risk factors. Age did not have a moderating effect on mortality or morbidity, i.e., young type A men did not show particularly elevated risks. Stress, as measured on the Reeder stress scale, did not appear to be an effect modifier either.

\subsection{Stress and Exhaustion}

\section{Reeder Stress Scale}

As discussed in Section 4.5, the analysis of stress was restricted to the Dutch cohort, since the Kaunas data were invalidated by a response set. Table 7.19 shows the age-adjusted relative risks of men who, according to the factor score distribution in tertiles, were categorized as experiencing stress to a moderate or severe extent, compared to men who did not experience stress. Stress, as measured by the Reeder stress scale, was clearly not associated with mortality and morbidity, as no risks were 
particularly increased. The use of continuous factor scores, instead of tertile scores, did not change this negative result.

Table 7.18 Age-adjusted association of the type A behavior with endpoints.

\begin{tabular}{|c|c|c|c|c|c|c|c|c|}
\hline & \multicolumn{2}{|c|}{ All-cause mortality } & \multicolumn{2}{|c|}{ CVD mortality } & \multicolumn{2}{|c|}{ CHD mortality } & \multirow[b]{2}{*}{ RR } & \multirow{2}{*}{$\begin{array}{r}\text { Nonfatal MI } \\
95 \% \mathrm{CI}\end{array}$} \\
\hline 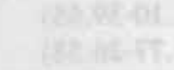 & RR & $95 \% \mathrm{CI}$ & RR & $95 \% \mathrm{Cl}$ & RR & $95 \% \mathrm{CI}$ & & \\
\hline 20 & nin & 1047: & & Kaunas & & & & \\
\hline Extreme B & 1.00 & F & 1.00 & & 1.00 & & 1.00 & \\
\hline Moderate B & 0.92 & $(0.60-1.41)$ & 1.30 & $(0.67-2.53)$ & 1.78 & $(0.77-4.13)$ & 1.24 & $(0.60-2.53)$ \\
\hline Moderate A & 1.34 & $(0.92-1.94)$ & 1.82 & $(1.01-3.29)$ & 2.32 & $(1.07-5.02)$ & 1.05 & $(0.50-2.20)$ \\
\hline Extreme A & 0.92 & $(0.65-1.29)$ & 0.78 & $(0.42-1.45)$ & 1.00 & $(0.45-2.23)$ & 1.01 & $(0.54-1.87)$ \\
\hline & & 7 & & Rotterdam & & & & \\
\hline Extreme B & 1.00 & 320 & 1.00 & & 1.00 & & 1.00 & \\
\hline Moderate B & 1.12 & $(0.79-1.60)$ & 0.91 & $(0.51-1.62)$ & 0.99 & $(0.53-1.87)$ & 0.86 & $(0.47-1.60)$ \\
\hline Moderate A & 0.93 & $(0.64-1.35)$ & 0.90 & (0.51-1.59) & 0.69 & $(0.34-1.41)$ & 0.85 & $(0.47-1.55)$ \\
\hline Extreme A & 1.00 & $(0.78-1.28)$ & 1.26 & $(0.89-1.80)$ & 1.29 & $(0.86-1.92)$ & 0.77 & $(0.50-1.17)$ \\
\hline
\end{tabular}

Table 7.19 Age-adjusted association of stress with endpoints in Rotterdam.

\begin{tabular}{|c|c|c|c|c|c|c|c|c|}
\hline & \multicolumn{2}{|c|}{ All-cause mortality } & \multicolumn{2}{|c|}{ CVD mortality } & \multicolumn{2}{|c|}{ CHD mortality } & \multicolumn{2}{|r|}{ Nonfatal MI } \\
\hline & RR & $95 \% \mathrm{CI}$ & RR & $95 \% \mathrm{CI}$ & RR & $95 \% \mathrm{CI}$ & $\mathbf{R R}$ & $95 \% \mathrm{CI}$ \\
\hline No stress & 1.00 & & 1.00 & & 1.00 & & 1.00 & \\
\hline Moderate & 0.88 & $(0.67-1.15)$ & 0.80 & $(0.54-1.19)$ & 0.89 & $(0.57-1.39)$ & 1.16 & $(0.74-1.82)$ \\
\hline Stress & 0.88 & $(0.67-1.14)$ & 0.88 & $(0.60-1.29)$ & 0.85 & $(0.54-1.33)$ & 1.08 & $(0.68-1.71)$ \\
\hline
\end{tabular}

\section{Exhaustion}

We refer again to item 7 of the Reeder stress scale : "At the end of the day I am completely exhausted mentally and physically". As explained in Section 4.5, it might indicate a breakdown in adaptation to stress and form a precursor of MI and cardiac death. As expected, among the men free of CHD-at-screening, a strong interaction between the level of exhaustion and the time between the start of the study and the occurrence of CHD mortality was found. This is shown in Table 7.20. In the first years of follow-up, those who were exhausted had much higher risks of CHD mortality than men who were not exhausted. In the first years the relative risk was 9.10. After 3-4 years the association was no longer statistically significant. It is, however, necessary to take care in drawing conclusions, as confidence intervals were very wide. 
Table 7.20 Age-adjusted association of exhaustion with CHD mortality for different lengths of follow-up among Dutch men without indications of CHD-at-screening.

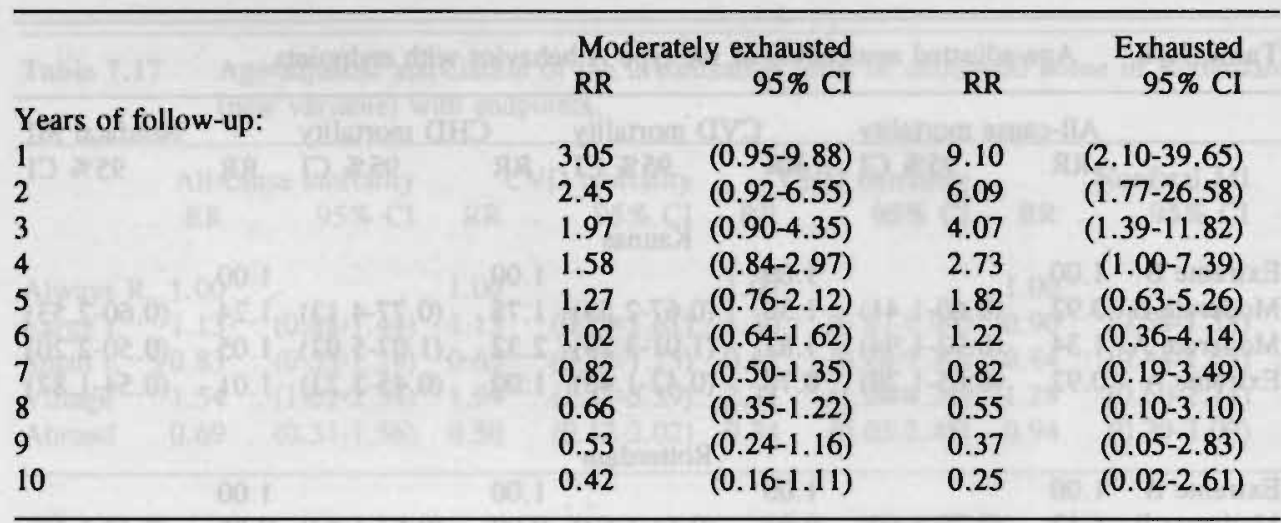

Table 7.21 shows that the exhausted men were, in general, somewhat older and leaner than the men who were not exhausted. They also were more often smokers and they were somewhat more frequently physically active.

Table 7.21 Distributions of biological and lifestyle risk factors for separate categories of exhaustion among Dutch men without indications of CHD-at-screening.

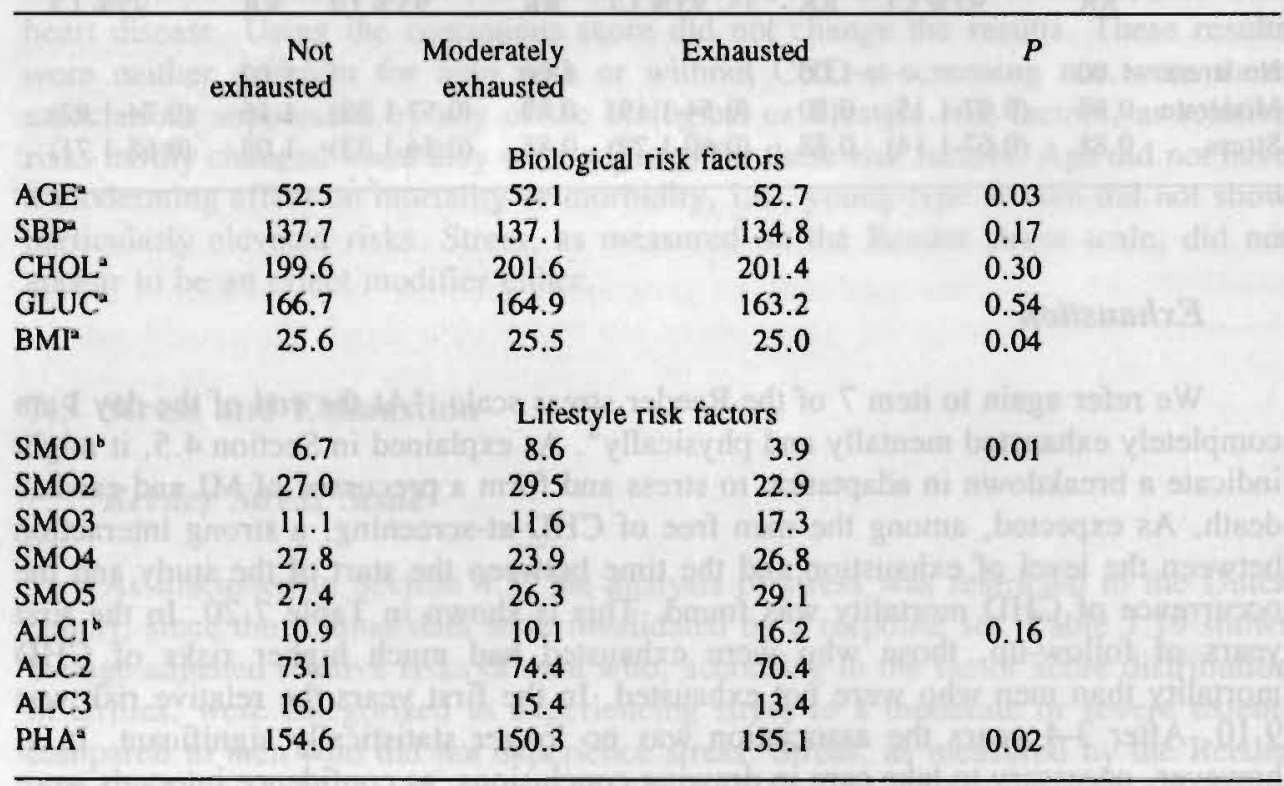

mean (F-test), ${ }^{b}$ percentage (chi ${ }^{2}$ test) 
The absence of any strong relevant associations of exhaustion with biological and lifestyle risk factors resulted in a multivariate model that is not much different from the age-adjusted model (Table 7.22).

Table 7.22 Association of exhaustion with CHD mortality for different lengths of follow-up, adjusted for all biological and lifestyle risk factors, among Dutch men without indications of CHD-at-screening.

\begin{tabular}{|c|c|c|c|c|}
\hline \multirow{2}{*}{ 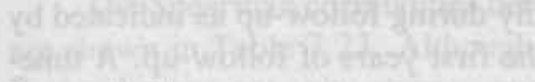 } & \multicolumn{2}{|c|}{ Moderately exhausted } & \multirow[b]{2}{*}{ RR } & \multirow{2}{*}{$\begin{array}{l}\text { Exhausted } \\
95 \% \mathrm{CI}\end{array}$} \\
\hline & RR & $95 \% \mathrm{CI}$ & & \\
\hline \multicolumn{5}{|c|}{ Years of follow-up: } \\
\hline 1 & 3.04 & $(0.97-9.78)$ & 8.68 & $(2.05-36.60)$ \\
\hline 2 & 2.53 & $(0.96-6.69)$ & 5.81 & $(1.82-18.54)$ \\
\hline 3 & 2.11 & $(0.96-4.62)$ & 3.89 & $(1.54-9.88)$ \\
\hline 4 & 1.76 & $(0.96-3.25)$ & 2.60 & $(1.17-5.81)$ \\
\hline 5 & 1.46 & $(0.91-2.36)$ & 1.74 & $(0.76-3.94)$ \\
\hline 6 & 1.22 & $(0.80-1.86)$ & 1.16 & $(0.44-3.10)$ \\
\hline 7 & 1.02 & $(0.64-1.62)$ & 0.78 & $(0.23-2.64)$ \\
\hline 8 & 0.85 & $(0.47-1.57)$ & 0.52 & $(0.12-2.36)$ \\
\hline 9 & 0.71 & $(0.32-1.54)$ & 0.35 & $(0.06-2.18)$ \\
\hline 10 & 0.59 & $(0.23-1.51)$ & 0.23 & $(0.03-2.01)$ \\
\hline
\end{tabular}

Figure 7.1 shows the probability of surviving the risk of CHD mortality during the whole follow-up period. Curves for the three exhaustion groups were adjusted for all biological and lifestyle risk factors by estimating the curves at the mean values of these risk factors. This figure clearly shows the elevated risks among exhausted men, particularly in the first years of follow-up.

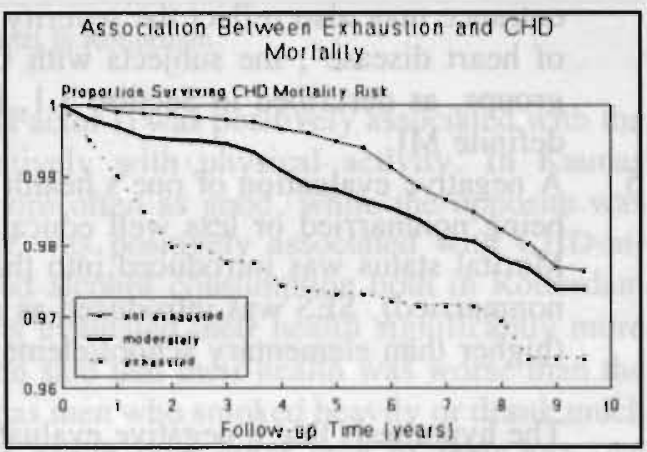

Figure 7.1 Survivors of risk of CHD mortality for separate categories of exhaustion, taking into account all risk factors.

\subsection{Self-rated Health}

As described in Section 4.6, four indicators were available for self-rated health: an evaluative factor (Factor I), a worry factor (Factor II), an evaluative question (Question I) and a comparative question (Question II). In search of an explanation for the association of a negative evaluation of one's health (as assessed by these four indicators) with mortality or morbidity, several hypotheses were explicitly tested: 
1. Self-assessment of health reflects knowledge of the parental health history. This hypothesis was tested by including the age at which the father and mother died in the models. Likewise, the self-assessment may reflect the health history of brothers or sisters. Therefore, the analysis was repeated with the inclusion of a variable showing whether a sibling had died.

2. A negative evaluation of one's health reflects an underlying subclinical disease, a prodromal condition of which the person is diffusely but not specifically aware. If an incipient condition were a confounding variable, the association of self-rated health with mortality should decrease markedly during follow-up as indicated by a significant drop of the relative risks after the first years of follow-up. A timedependent covariate was used to test whether the relative risks are constant during follow-up.

3. A negative evaluation of one's own health is based upon symptoms of ill health not asked for in routine medical examinations, but which make a subject worry about his health. This hypothesis was tested by adjusting the predictive power of the evaluative questions for an index reflecting the extent to which people worry about their health (Factor II).

4. A negative evaluation of one's own health may reflect manifest disease. As before, we adjusted for the presence of heart disease by including this variable in all analyses. In addition, the analyses were repeated excluding the group with CHD-at-screening.

A negative evaluation of one's health among those with manifest heart disease at entrance may also reflect the severity of the disease. To adjust for the "severity of heart disease", the subjects with CHD-at-screening were divided into three groups, as described in Section 3.1, i.e., angina pectoris, abnormal ECG, and definite MI.

5. A negative evaluation of one's health reflects difficult living conditions such as being nonmarried or less well educated. These factors are taken into account. Marital status was introduced into the models as a dummy variable (married/nonmarried). SES was introduced as a dummy variable of the educational level (higher than elementary school/elementary school only).

The hypothesis that a negative evaluation of one's health reflects a low sense of mastery was tested by computing Spearman correlations. For this purpose, the two factors of the semantic differential test and the two direct questions were correlated with some selected items from the baseline interview. These items express the belief that good health is not only a matter of good or bad luck but is to some extent under one's own control. The selected items were: (1) "Do you think a healthy person of your age could reduce the likelihood of developing some serious disease by taking preventive measures?" (very likely, likely, not likely) and (2) "People have different opinions about health and disease. With which of these statements do you agree?" (if one gets ill, it can't be helped; generally health is a matter of luck, but in some cases we can do things to prevent development of diseases; one can certainly do many things to improve one's health status). We also selected two adjective pairs of a semantic 
differential test for "Heart Disease". The selected pairs were: "I can do much .. little about it": "it depends on me ... it depends on luck". To interpret the correlations of these items more easily, some answer categories were recoded to give all of them similar directions.

\section{Results of Analyses}

The Spearman correlations between the two direct questions and the factor scores are shown in Table 7.23. Although the correlations between the direct questions and Factor I were not very strong, one can say that they shared a global evaluation of one's health. Factor II had rather low correlations with the other indicators. This indicates that Factor II was conceptually different from the other indicators.

Table 7.23 Spearman correlation coefficients among the indicators of self-rated health".

\begin{tabular}{lrrrr}
\hline & Factor I & Factor II & Question I & Question II \\
Factor I & & 0.20 & 0.53 & 0.45 \\
Factor II & 0.15 & 0.29 & 0.35 & 0.27 \\
Question I & 0.53 & 0.28 & 0.50 & 0.54 \\
Question II & 0.51 & & \\
\hline
\end{tabular}

"Above the diagonal is Kaunas; beneath the diagonal is Rotterdam.

In both cohorts the evaluative factor (Factor I) was positively associated with the presence of CHD-at-screening and negatively with physical activity. In Kaunas smokers rated their health significantly more often as good, while the opposite was observed in Rotterdam. The worry factor was positively associated with CHD-atscreening and negatively with smoking and alcohol consumption both in Rotterdam and Kaunas. Those with CHD-at-screening evaluated their health significantly more often as poor on Question I and more often said that their health was worse than the health of other men of their own age. Kaunas men who smoked heavily or drank much alcohol reported that they were in better health than other men of their age. An opposite relation was observed in Rotterdam.

The four indices of self-assessed health were almost equally associated with mortality in men with or without CHD-at-screening. However, the comparative question (Question II) was much more strongly associated with all-cause mortality among Dutch men with indications of CHD than among men without CHD-atscreening. Therefore, these groups were not combined in the analysis of that question for all-cause mortality in Rotterdam.

In both cohorts those men who evaluated their health as "poor" on Factor I were at elevated risk of mortality compared to those who evaluated their health as good. The relative risks were 1.52 in Kaunas and 2.07 in Rotterdam (Table 7.24). 
Table 7.24 Age-adjusted association of self-rated health with all-cause mortality, adjusted for CHD-at-screening (Model I) and adjusted for all biological and lifestyle risk factors (Model II).

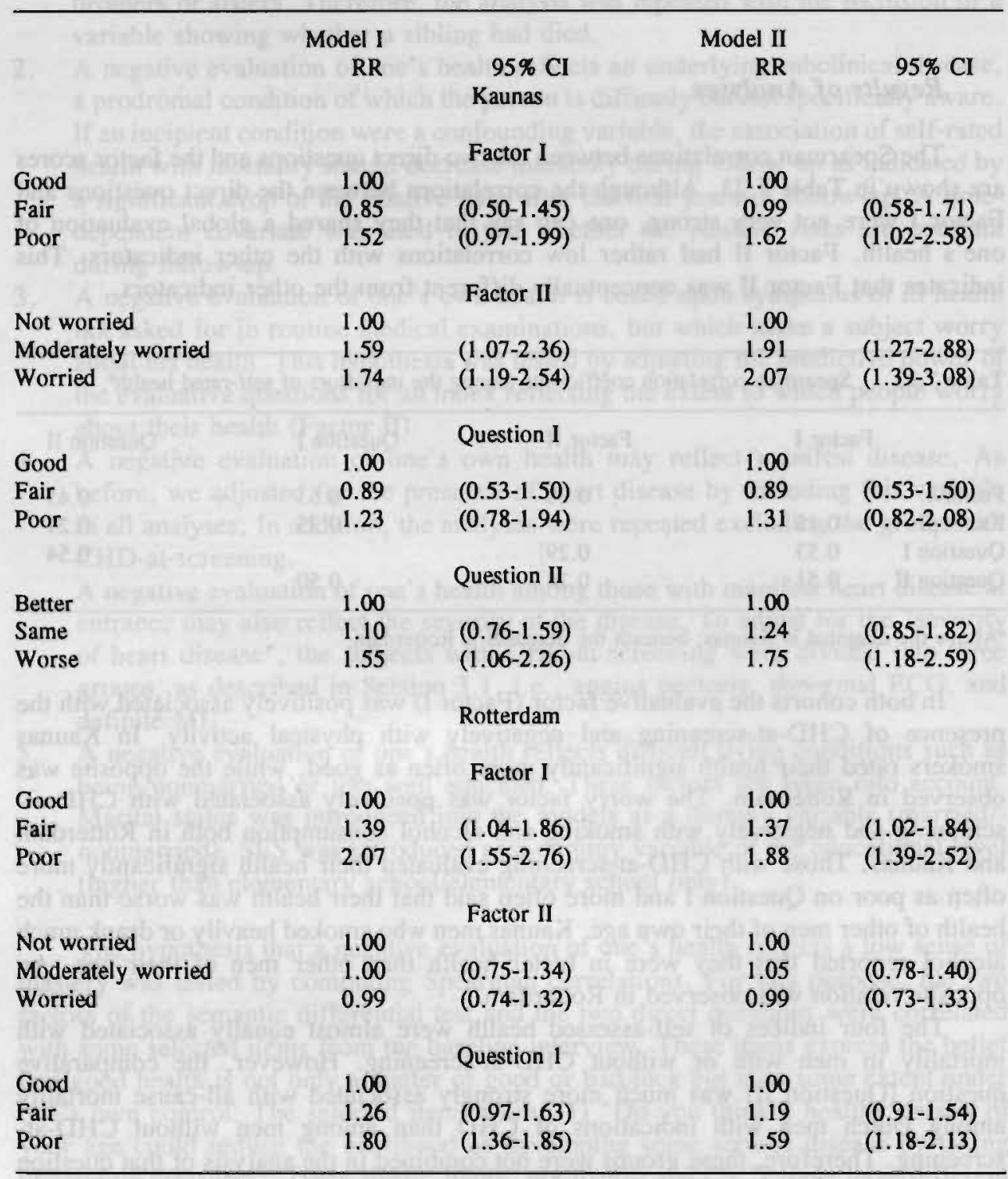

In Rotterdam the association was highly significant, while in Kaunas it just failed to reach the conventional 0.05 level of statistical significance. Adjusting for risk factors (model II) did not change these effects meaningfully. In Kaunas a slight 
increase (resulting in statistical significance) of the relative risk was observed ( $R R=$ $1.62)$, while a decrease was observed in Rotterdam $(R R=1.88)$. Detailed analysis shows that these changes were accounted for by smoking behavior, which was differently associated with Factor I in the two cohorts.

Factor II is significantly associated with mortality in Kaunas $(R R=1.74)$ but lacks any association with mortality in Rotterdam $(R R=0.99)$. Taking the classic risk factors into account resulted in an increase of the relative risk in Kaunas, because Lithuanians who worried about their health smoked less than others.

Question I ("How would you assess your own health?") was not associated with mortality in Kaunas ( $R R=1.23$ ), while in Rotterdam the mortality risk of men who assessed their health as poor was increased by $80 \%(R R=1.80)$. Adjusting for risk factors reduced this excess risk to $59 \%$, but it remained highly significant. The decrease was mainly due to the fact that smokers more often rated their health as poor.

In both cohorts the comparative question (Question II) predicted mortality. Lithuanians who said that their health was worse than that of other men of their age had an increased risk of $55 \%(R R=1.55)$ (Table 7.24). This relative risk increased in model II to 1.75 , mainly because those who said that their health was worse than that of other men smoked less often. For the Dutch without CHD-at-screening, the mortality risk of those who said that their health was worse than that of other men of their age was increased by $79 \%$ (Model I in Table 7.25). This excess risk was reduced to $54 \%$ in model II, but remained significant. This decrease was mainly due to smoking. Among Dutch men with CHD-at-screening the comparative question (Question II) was strongly associated with mortality. The age-adjusted relative risk decreased from 5.59 to 5.21 , when the traditional risk factors were taken into account, but remained highly significant.

Table 7.25 Association of Question II with all-cause mortality, separately for men with and without CHD-at-screening in Rotterdam, adjusted for age (Model I) and adjusted for all biological and lifestyle risk factors (Model II).

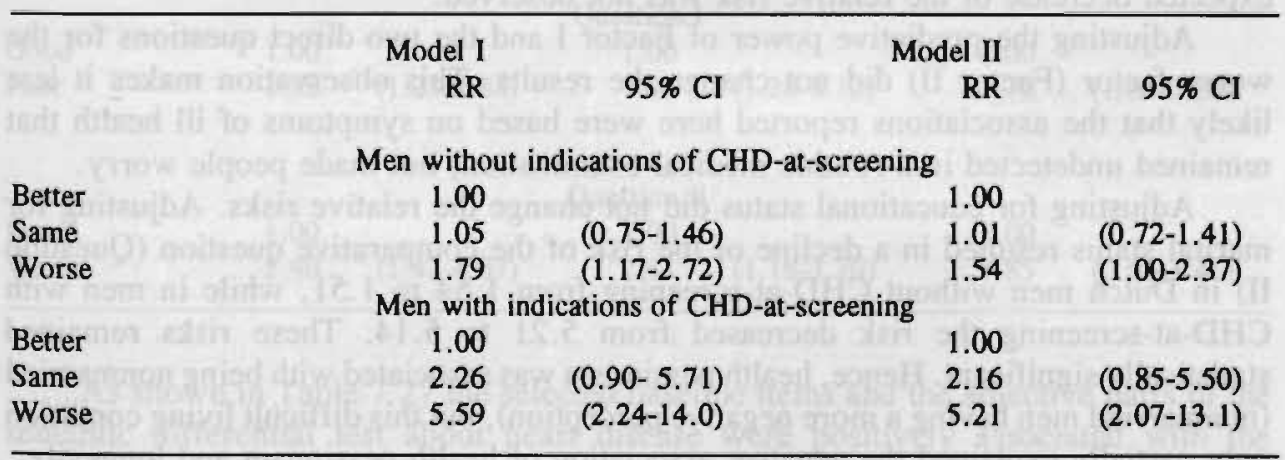

Separate analysis for the cardiovascular endpoints showed that Factor I is especially associated with CHD mortality in Rotterdam (Table 7.26). Factor II was 
strongly associated with CVD mortality in Kaunas. The evaluative question (Question I) was predictive of mortality due to CVD and CHD in Rotterdam. The comparative question (Question II) was associated with CVD and CHD mortality in both cohorts. The two direct questions appeared to be associated with nonfatal MI as well, but only in Rotterdam. The relative risks were 1.70 and 1.85 , respectively. Hence, the general impression is that a negative evaluation of one's health was associated with mortality through its association with CVD.

The main results show that a poor perception of one's health had a negative association with longevity both among the apparently healthy and among those with signs of CHD. Was this association spurious because of confounding factors?

The addition to model II of the age at which the father or mother died did not change the results meaningfully. Only the relative risk of the comparative question (Question II) among the Rotterdam participants with CHD-at-screening decreased from 5.21 to 4.36 , but remained statistically significant. Inclusion of a variable showing whether one of the siblings had died in model II did not change the relative risks. These results make it rather unlikely that a negative perception of one's health was associated with mortality because a gloomy answer reflected knowledge of the parental or familial longevity.

Did an unfavorable answer reflect a disease that was not yet clinically manifest? We observed that the relative risks decreased slightly during follow-up, but the decrease was never significant. Furthermore, adjusting for the severity of CHD-atscreening resulted in a slight increase of the risk among those who rated their health as poor on Factor I in both cohorts. The results of Factor II did not change. A marked increase of the risk associated with a gloomy answer on the evaluative question (Question I) was observed in Kaunas ( $R R=1.62 ; P=<0.05$ ), while a slight decrease was observed in Rotterdam (RR $=1.61 ; P<0.05$ ). Concerning the comparative question (question II) a slight increase in the relative risk was observed both in Kaunas and Rotterdam. Given the poor validity of the index of "severity of heart disease", this result should be interpreted carefully. All we can say is that the expected decrease of the relative risk was not observed.

Adjusting the predictive power of Factor I and the two direct questions for the worry factor (Factor II) did not change the results. This observation makes it less likely that the associations reported here were based on symptoms of ill health that remained undetected in a routine medical examination, but made people worry.

Adjusting for educational status did not change the relative risks. Adjusting for marital status resulted in a decline of the risk of the comparative question (Question II) in Dutch men without CHD-at-screening from 1.54 to 1.51 , while in men with CHD-at-screening the risk decreased from 5.21 to 5.14 . These risks remained statistically significant. Hence, health pessimism was associated with being nonmarried (nonmarried men having a more negative perception), but this difficult living condition only partially explained the negative association of health pessimism and longevity. 
Tuble 7.26 Association of self-rated health with CVD mortality, CHD mortality and nonfatal MI, adjusted for all biological and lifestyle risk factors.

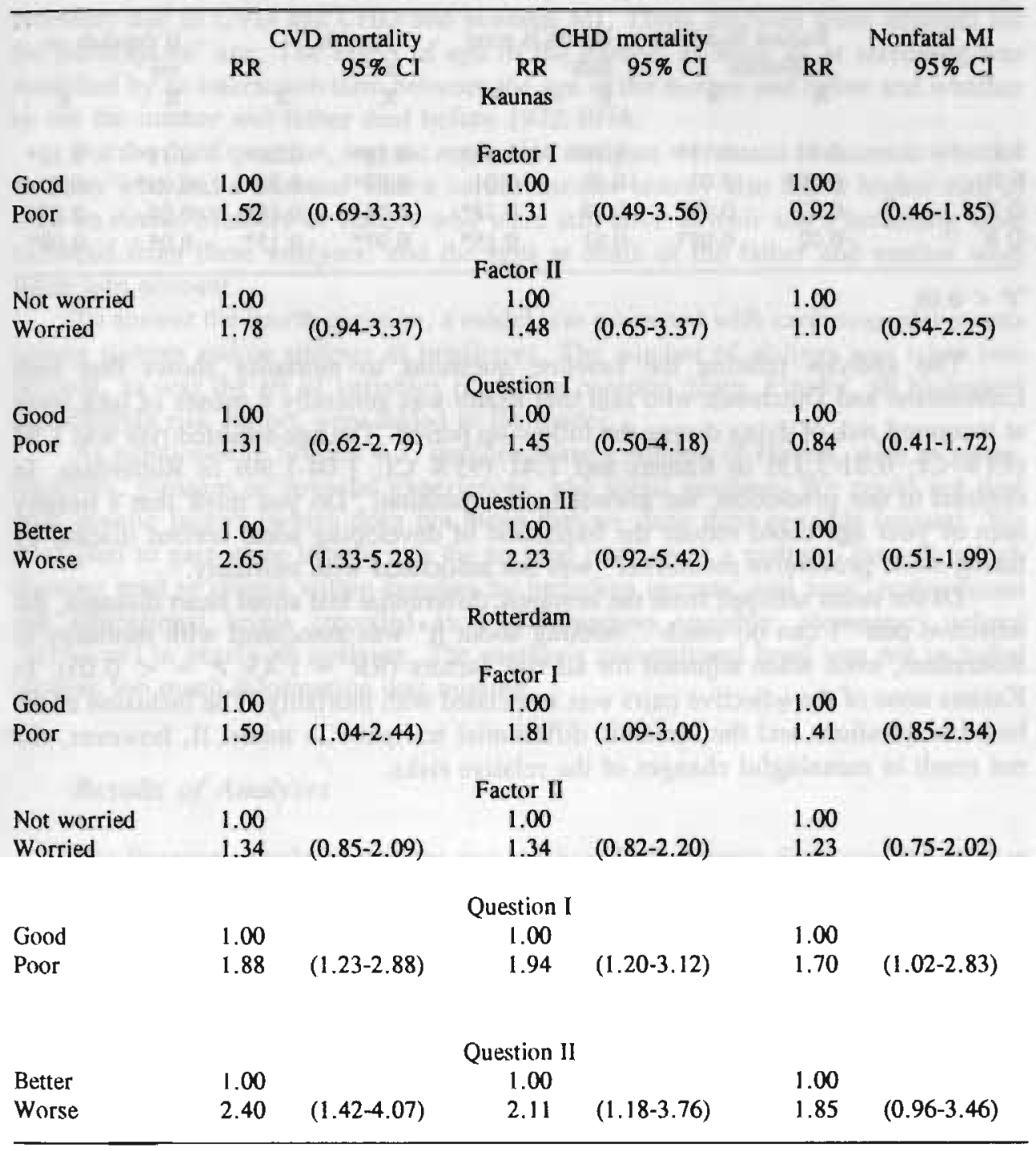

As shown in Table 7.27 the selected baseline items and the adjective pairs of the semantic differential test about heart disease were positively associated with the evaluative factor and the two direct questions. The absolute size of these correlations was rather modest, especially in Kaunas. Nonetheless, they give support to the belief that a positive evaluation of one's health was associated with the general feeling that one can do things to improve one's health. 
Spearman correlations of indicators of self-rated health with selected baseline items and items from the semantic differential test about heart disease.

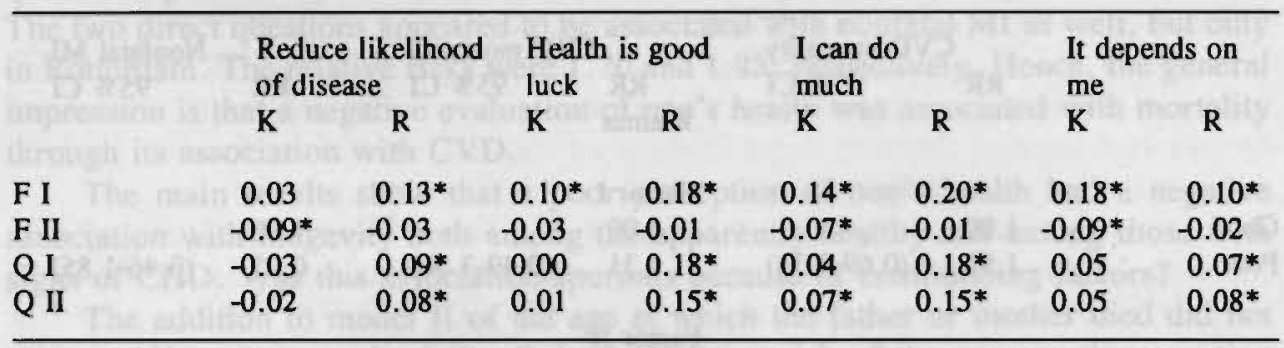

$\cdot P<0.05$

The analysis relating the baseline questions to mortality shows that both Lithuanians and Dutchmen who said that health was generally a matter of luck were at increased risk of dying during the follow-up period. The age-adjusted risk was 1.59 (95\% CI: 0.81-3.13) in Kaunas and 1.41 (95\% CI: 1.04-1.90) in Rotterdam. In contrast to our prediction, the answer to the question: "Do you think that a healthy man of your age could reduce the likelihood of developing some serious disease by taking some preventive measures?" was not associated with mortality.

Of the items selected from the semantic differential test about heart diseases, the adjective pair "I can do much ...nothing about it" was associated with mortality in Rotterdam, even when adjusted for all risk factors ( $R R=1.43 ; P=<0.05$ ). In Kaunas none of the adjective pairs was associated with mortality. The inclusion of the baseline questions and the semantic differential test pairs in model II, however, did not result in meaningful changes of the relative risks.

\subsection{Family Aggregation of CVD}

Answers were sought for the four following questions:

1. Is the life expectancy of the parents correlated?

2. Is parental longevity correlated with the life expectancy of the participants?

3. Are women whose husbands experienced a coronary event at increased risk of CHD?

4. Are participants who had a parent or a sibling who experienced a cardiovascular event at increased risk of CHD?

Analyses regarding questions 3 and 4 were restricted to men without indications of CHD-at-screening. This exclusion was applied to avoid recall bias in answering questions regarding a family history of CVD. Excluding those men with an old MI, abnormal ECG and/or angina pectoris, analyses were done for 2044 men in Kaunas and 2909 men in Rotterdam.

To answer the first question, the ages at death of both parents were correlated using Pearson correlations. To answer the second question, correlations were 
computed between age at death of KRIS participants and their father's or mother's age at death. Furthermore, ages at death of parents or parental ages at screening, if alive, were analyzed for their association with the participants' risk of all-cause mortality, mortality due to CVD and CHD and nonfatal MI. These analyses were adjusted for the participants' age. The effect of age of the parents at death or at screening was modelled by an interaction term between the age of the mother and father and whether or not the mother and father died before 1972-1974.

For the third question, logistic regression analyses were used to examine whether mothers who had a husband with a cardiovascular history also had a higher risk of such an event. Mothers or fathers who were still alive at their son's screening were excluded from these analyses, and the ages at death of the father and mother were taken into account.

To answer the fourth question, a model was estimated with cardiovascular events among parents and/or siblings as predictors. The number of siblings was taken into account, as was the set of variables regarding parental death. Finally, all biological and lifestyle risk factors were added to the model.

As mentioned in Section 4.7, families share a number of factors, such as genes, lifestyles, pleasant or stressful experiences, and social position. We could not deal with genetic factors (which does not mean that we deny their possible impact). We attempted to gain more insight into the societal position as a pathway through which diseases tend to cluster within families by including parents' and sons' occupational and educational levels (recoded as a dichotomous variable: elementary school only/more) in nearly all analyses. The mother's occupational level was not included because too much information was missing.

\section{Results of Analyses}

The Pearson correlations of the age at which both parents died were $0.14(P=$ 0.00 ) in both cities. Adjusting for similarities in educational level did not change the correlation coefficients. Hence, the age at death of one parent explained approximately $2 \%$ of the variance of the lifespan of the other parent $\left(r^{2}=0.02\right)$.

The correlation coefficients between the age at death of deceased participants and the age at death of their fathers were -0.06 in Rotterdam and 0.23 in Kaunas. The respective figures were 0.08 and 0.11 for the age at death of their mothers. Table 7.28 shows the risks of all-cause mortality associated with the parental age when they died, or at screening if they were alive. The effects are very small or absent and no particular pattern was apparent. When no interaction was modelled, the main effects were not significant either. This negative picture did not change when the cardiovascular endpoints were investigated. 
Table 7.28 Age-adjusted association of age of parents at death or at screening, if alive, with allcause mortality.

\begin{tabular}{lrrrrr}
\hline & RR & $\begin{array}{r}\text { Kaunas } \\
95 \% \text { CI }\end{array}$ & RR & $\begin{array}{r}\text { Rotterdam } \\
95 \% \text { CI }\end{array}$ \\
Father alive & 0.99 & $(0.94-1.04)$ & 1.02 & $(0.97-1.07)$ \\
Father dead & 1.01 & $(0.96-1.06)$ & 1.00 & $(0.95-1.05)$ \\
Mother alive & 1.02 & $(0.99-1.06)$ & 0.97 & $(0.97-1.04)$ \\
Mother dead & 1.00 & $(0.97-1.03)$ & 1.00 & $(0.96-1.04)$ \\
\hline
\end{tabular}

Table 7.29 presents the lifetime odds ratios of a cardiovascular event among the wives of men with or without a positive cardiovascular history. In Rotterdam, the risk among mothers was only marginally elevated. Educational similarities between spouses did not influence the results. The fathers' cardiovascular history was substantially associated with their wives' lifetime risk of such an event in Kaunas $(O R=1.71)$. This was based partly on similarities in their educational levels, as suggested by the slight decrease of the odds ratio, when adjusted for educational level.

Table 7.29 Association (odds ratios) of mothers' lifetime risk of a cardiovascular event with fathers' risk of such an event, adjusting for age of parents at death (Model I) and: age of parents at death and educational level of parents (Model II).

\begin{tabular}{lrrrrr}
\hline & OR & $\begin{array}{r}\text { Kaunas } \\
95 \% \mathrm{CI}\end{array}$ & OR & $\begin{array}{r}\text { Rotterdam } \\
95 \% \mathrm{CI}\end{array}$ \\
I & 1.71 & $(1.11-2.61)$ & 1.08 & $(0.83-1.41)$ \\
II & 1.56 & $(1.00-2.43)$ & 1.09 & $(0.82-1.45)$
\end{tabular}

- Men with indications of CHD were excluded. In Model II 947 fathers of whose wives 161 had a cardiovascular event were analyzed in Kaunas; corresponding numbers in Rotterdam were: 1264 and 326.

Only 77 Kaunasians had one or more siblings who had had a cardiovascular event. Of these Kaunas participants only three died of CVD and one died of CHD during follow-up. This resulted in severe problems of convergence in the Cox proportional hazards model. Therefore, analyses in Kaunas were restricted to parental history of cardiovascular events. Table 7.30 shows the effects of cardiovascular events within families on the participant's risk of several endpoints. As this table shows, strong effects of a positive parental history were found for the risk of nonfatal MI, the relative risks being 3.03 and 1.71 in Kaunas and Rotterdam, respectively. Adjusting for the siblings with a cardiovascular history and the number of siblings only marginally decreased the relative risks in Rotterdam. Independent of their parental cardiovascular history, Rotterdam participants with siblings having a positive 
cardiovascular history had highly elevated mortality and morbidity risks during followup compared to those without such siblings (RR $>2$ for all three cardiovascular endpoints). The interaction between a parental history of CVD and CVD among siblings was not statistically significant. The relative risks for men whose siblings had a history of CVD were, thus, similar for men with and without a parental history of CVD.

Table 7.30 Association of a history of cardiovascular events among parents and siblings with endpoints, adjusted for age (Model I) and adjusted for age and each other (Model II)".

\begin{tabular}{|c|c|c|c|c|c|c|c|c|}
\hline & \multicolumn{2}{|c|}{ All-cause mortality } & \multicolumn{2}{|c|}{ CVD mortality } & \multicolumn{2}{|c|}{ CHD mortality } & \multicolumn{2}{|r|}{ Nonfatal MI } \\
\hline & RR & $95 \% \mathrm{CI}$ & RR & $\begin{array}{l}95 \% \mathrm{CI} \\
\text { Kaunas }\end{array}$ & RR & $95 \% \mathrm{Cl}$ & RR & $95 \% \mathrm{CI}$ \\
\hline Parent & 1.00 & $(0.73-1.38)$ & 1.22 & $(0.68-2.20)$ & 1.77 & $(0.88-3.57)$ & 3.03 & $(1.60-5.72)$ \\
\hline & knt & & & $\begin{array}{c}\text { Rotterdam } \\
\text { Model I }\end{array}$ & & & & \\
\hline Parent & 0.84 & $(0.64-1.10)$ & 1.05 & $(0.69-1.59)$ & 1.23 & $(0.77-1.96)$ & 1.71 & $(1.12-2.61)$ \\
\hline Siblings & 1.40 & $(1.01-1.95)$ & 2.33 & $(1.45-3.73)$ & 2.11 & $(1.22-3.65)$ & 2.27 & $(1.38-3.71)$ \\
\hline & $x^{2}$ & (c) & & Model II & & & & \\
\hline Parent & 0.84 & $(0.64-1.96)$ & 1.04 & $(0.68-1.59)$ & 1.22 & $(0.76-1.96)$ & 1.59 & $(1.04-2.43)$ \\
\hline Siblings & 1.42 & $(1.01-1.99)$ & 2.33 & $(1.45-3.76)$ & 2.09 & $(1.20-3.63)$ & 2.06 & $(1.25-3.41)$ \\
\hline
\end{tabular}

Table 7.31 shows the mean values or percentages of the biological and lifestyle risk factors of those with and without a family history of CVD. Associations with family aggregation were statistically significant for age, systolic blood pressure, cholesterol level, glucose tolerance, physical activity, and body mass index. Blood pressure, cholesterol level, glucose tolerance and body mass index appeared to be higher for those with a family history. Noteworthy are the higher levels among Dutch participants with siblings with a cardiovascular history compared to participants who had a parental cardiovascular history.

The model in which a family history was simultaneously adjusted for all biological and lifestyle risk factors is shown in Table 7.32. The relative risks declined only marginally when these covariates were adjusted. The small decline was particularly due to high blood pressure and cholesterol level among those males with a family history of CVD.

The occupational and educational levels of the parents and their children give an indication of the influence of a family's social position. The analysis about this influence shows that the influence of a positive family history of CVD could not be explained by similarities in social position between generations or between siblings during their upbringing. 
Table 7.31 Distributions of biological and lifestyle risk factors for separate categories of family history of CVD.

\begin{tabular}{|c|c|c|c|c|c|c|c|c|}
\hline \multirow{2}{*}{\multicolumn{4}{|c|}{$\begin{array}{c}\text { Kaunas } \\
\text { Parental history }\end{array}$}} & & & & \multicolumn{2}{|c|}{ Rotterdam } \\
\hline & & & & & & Family his & & \\
\hline \multicolumn{7}{|c|}{ 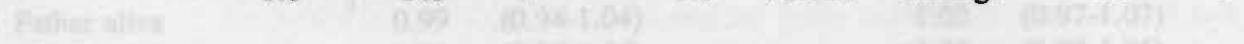 } & both & $P$ \\
\hline \multicolumn{9}{|c|}{ Biological risk factors } \\
\hline $\mathrm{AGE}^{\mathrm{a}}$ & 52.1 & 51.5 & 0.01 & 52.2 & 52.1 & 53.3 & 52.9 & 0.00 \\
\hline SBP & 135.9 & 139.3 & 0.00 & 136.6 & 137.2 & 139.5 & 138.7 & 0.15 \\
\hline $\mathrm{CHOL}^{*}$ & 191.4 & 192.2 & 0.66 & 199.3 & 200.5 & 201.5 & 207.0 & 0.02 \\
\hline GLUC & 142.9 & 147.4 & 0.10 & 163.4 & 167.7 & 169.3 & 171.2 & 0.06 \\
\hline $\mathrm{BMI}^{\mathrm{P}}$ & 25.7 & 26.2 & 0.01 & 25.5 & 25.5 & 25.7 & 26.0 & 0.12 \\
\hline \multicolumn{9}{|c|}{ Lifestyle risk factors } \\
\hline $\mathrm{SMO1}^{\mathrm{b}}$ & 27.6 & 29.5 & 0.74 & 7.8 & 7.5 & 8.8 & 4.6 & 0.87 \\
\hline $\mathrm{SMO} 2$ & 24.0 & 21.7 & & 27.9 & 30.0 & 25.3 & 30.1 & \\
\hline SMO3 & 9.0 & 8.2 & & 12.1 & 11.2 & 10.8 & 12.2 & \\
\hline SMO4 & 14.6 & 16.2 & & 25.7 & 24.1 & 28.4 & 27.0 & $=$ \\
\hline SMO5 & 24.9 & 24.4 & & 26.5 & 27.2 & 26.8 & 26.0 & 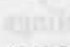 \\
\hline $\mathrm{ALC1}^{\mathrm{b}}$ & 2.0 & 1.3 & 0.34 & 10.4 & 10.2 & 14.5 & 13.3 & 0.28 \\
\hline $\mathrm{ALC} 2$ & 64.9 & 68.3 & & 74.1 & 74.4 & 72.5 & 67.9 & \\
\hline ALC3 & 33.1 & 30.5 & & 15.5 & 15.4 & 13.0 & 18.9 & \\
\hline $\mathrm{PHA}^{a}$ & 201.3 & 212.2 & 0.00 & 152.6 & 151.0 & 155.3 & 152.0 & 0.58 \\
\hline
\end{tabular}

- mean (F-test), ${ }^{b}$ percentage (chi ${ }^{2}$ test)

Table 7.32 Association of a history of cardiovascular events among parents and siblings with endpoints, adjusted for all biological and lifestyle risk factors (in Rotterdam, number of siblings was also adjusted for).

\begin{tabular}{|c|c|c|c|c|c|c|c|c|}
\hline & \multicolumn{2}{|c|}{ All-cause mortality } & \multicolumn{2}{|c|}{ CVD mortality } & \multicolumn{2}{|c|}{ CHD mortality } & \multicolumn{2}{|r|}{ Nonfatal MI } \\
\hline & RR & $95 \% \mathrm{CI}$ & RR & $\begin{array}{l}95 \% \mathrm{Cl} \\
\text { Kaunas }\end{array}$ & RR & $95 \% \mathrm{CI}$ & RR & $95 \% \mathrm{CI}$ \\
\hline Parent & 1.02 & $(0.73-1.42)$ & 1.17 & $(0.64-2.14)$ & 1.61 & $(0.79-3.29)$ & 2.48 & $(1.29-4.76)$ \\
\hline & & & & Rotterdan & & & & \\
\hline Parent & 0.84 & $(0.64-1.10)$ & 1.01 & $(0.66-1.54)$ & 1.19 & $(0.74-1.92)$ & 1.52 & $(0.99-2.35)$ \\
\hline Siblings & 1.43 & $(1.02-2.01)$ & 2.14 & $(1.32-3.05)$ & 1.98 & $(1.14-3.47)$ & 1.95 & $(1.17-3.26)$ \\
\hline
\end{tabular}

\subsection{Additional Predictive Value of Psychosocial Risk Indicators}

The data with regard to SES, marital status, geographic mobility, exhaustion, self-rated health, and family history of CVD support the general belief that psychoso- 
cial factors merit the attention of preventive cardiology. Each of them is associated with (cardiovascular) mortality.

One may ask, however, how much they contribute to the identification of high risk subjects. This question has little importance for the study of the etiological pathways (because it does not consider the direction of the associations between psychosocial and classic risk factors). However, it is of major importance for designing cost-efficient intervention strategies in public health policy.

Receiver Operator Characteristic (ROC) curves provide a handy tool for investigating the increase of the predictive power of a set of tests when a new test is added (Sackett et al., 1991). This method was developed for the evaluation of the sensitivity and the specificity of diagnostic tests. It can also be used to investigate the relative discriminatory power of the determinants of a disease. With the use of the logistic regression solution, probabilities of mortality or disease can be calculated for each individual. Thereafter, a specific cutoff point can be selected to classify subjects as those who are predicted to develop a disease and those who are not. The sensitivity and specificity of the test can then be calculated by cross-tabulating the predicted and observed number of cases and noncases. Sensitivity is the probability that a case is predicted to be a case on the basis of entry characteristics. Specificity is the probability that an observed noncase is predicted to be a noncase. It is intuitively clear that the sensitivity and specificity of a test are related to the cutoff point used. ROC curves combine both statistics along all cutoff points. They plot the sensitivity as a function of 1 - specificity. Good discriminatory power (high sensitivity and specificity) is achieved when the ROC curve approaches the upper left-hand corner of the figure.

The computation of the gain in discriminatory power when the psychosocial factors are added to all biological and lifestyle risk factors requires a model in which all variables are included. Attempts to do so resulted in an overfitted model, in which there were too few participants, given the number of variables. Since the data for many psychosocial risk indicators were missing, the sample sizes became extremely small when several psychosocial factors were analyzed simultaneously. The Dutch cohort was reduced to $67 \%$ and the Lithuanian cohort to $37 \%$ of its original size, when occupational level, geographic mobility, marital status, self-rated health, and a parental history of CVD were analyzed simultaneously. This resulted in problems of convergence due to empty cells. Therefore, it was decided to pool the cohorts for this analysis. Three psychosocial risk indicators were similarly measured in both cohorts and related to the endpoints in this pooled data set: marital status, self-rated health, and a parental history of CVD. A composite score of self-rated health was constructed by adding Factor I and Question I and II. To decrease the number of data missing on this variable, missing observations for the continuous factor score were estimated by regressing (ordinary least squares) this factor score on the two questions (with dichotomized variables). Although interactions between the cohort and any of the risk factors and indicators were never statistically significant, the relative risks in this pooled cohorts data set clearly reflected a mixture of both Dutch and Lithuanian experiences. In the analysis with the pooled data set, nationality was defined by the variable "cohort" [(0) Rotterdam and (1) Kaunas]. Due to this reorganization of the 
data set and the reduction of the number of psychosocial factors, the analysis is mainly a methodological exercise.

The ROC curves for the four endpoints are shown in Figure 7.2. In each figure the lowest line represents the discriminatory power of a model with age and cohort. Choosing 1-specificity $=20 \%$ as an optimal level of specificity, the figure indicates that the sensitivity for all-cause mortality is $32 \%$, and for CHD mortality $30 \%$ at this point. Adding the psychosocial factors to this model (second line from the bottom in Figure 7.2 ) increased these percentages to $40 \%$ and $41 \%$. Adding the eight biological and lifestyle factors to the model increased the sensitivity to $53 \%$ and $57 \%$, respectively. When all risk factors were included in the same model, the sensitivity increased to $60 \%$ and $61 \%$, respectively.

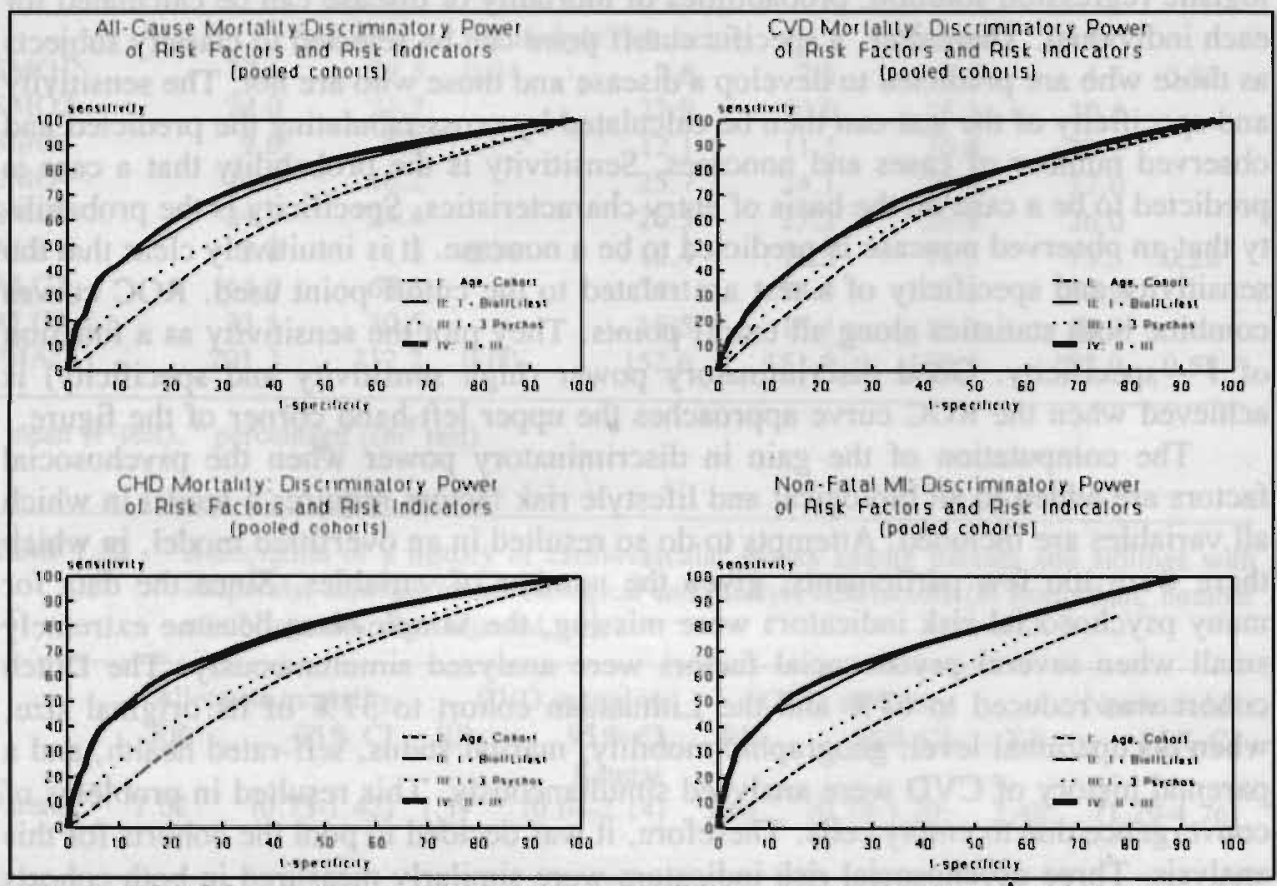

Figure 7.2 ROC curves: age, cohort, biological and lifestyle risk factors and marital status, selfrated health and a parental history of CVD.

Given the fact that only a limited number of psychosocial factors could be included in the analysis (and the debatable inclusion of a positive family history as a psychosocial factor), the absolute numbers should be interpreted cautiously. Nevertheless, the methodological exercise illustrates several points. Compared to the "baseline" discriminatory power of age and cohort, the selected psychosocial factors added meaningfully to the risk profile. The largest gain in discriminatory power was obtained 
by the classic risk factors. Adding the psychosocial factors to the biological and lifestyle risk factors resulted in a modest increase of discriminatory power.

\subsection{Summary and Discussion}

\section{SES}

A low occupational status was found to increase the risk of all-cause mortality in both cohorts significantly. Cardiovascular endpoints also tended to occur more often in lower occupational groups, especially in Rotterdam. Adjusting for all biological lifestyle risk factors resulted in a slight increase of the relative risks for the cardiovascular endpoints in Kaunas, mainly because mean levels of cholesterol were lower in the lowest stratum. However, the strength of the associations did not reach conventional levels of statistical significance. Adjustment for these risk factors in Rotterdam resulted in a small decline of the associations. This was mainly due to the higher prevalence of smoking in the lower occupational groups. The level of education was found to be associated with all-cause mortality in Rotterdam. No other significant associations were observed.

The precision of the relative risks may be biased by several factors. First, as mentioned in section 2.3 , nonparticipants of the screening examinations more often came from lower socioeconomic groups and had higher mortality risks. This may have resulted in conservative estimates of relative risks. Secondly, the measurement of occupational level in Kaunas was rather crude because detailed information about occupations was classified information in the early 1970s. Those closely affiliated with the communist party could not be differentiated. This may have affected the results because this group possibly had more access to medical services (Connor, 1979; Mezentseva \& Rimachevskaya, 1992; Wnuk-Lipinski \& Illsley, 1990).

A more fundamental question is whether the social stratification underlying the SES-mortality gradient in western countries has the same meaning in socialist countries, because it assumes a societal hierarchy in the possession of scarce goods. In socialist countries the social stratification is not based on market mechanisms, but on the control of resources (Mezentzeva \& Rimachevskaya, 1992). Affiliation with the communist party, then, becomes an important factor for the kind and amount of scarce resources and services obtained (Connor, 1979; Matthews, 1972; Ultee et al., 1992; Wnuk-Lipinski \& Illsley, 1990). The correlation between professional skills/educational level and income is much weaker in socialist countries than in western countries. This is illustrated by the relatively low incomes of those working in the educational or health sector. These sectors are considered unproductive in contrast to the heavy industry or the army. Nevertheless, the distinction between employee and worker probably reflects a manual/nonmanual hierarchy, which has some impact on the possession of scarce goods (income, power, prestige, knowledge) that resembles that of capitalist countries (Connor, 1979; Ultee et al., 1992). This 
implies that it is reasonable to investigate the association of SES and health in western and socialist countries according to similar lines of reasoning.

Multiple causal pathways have been proposed to explain socioeconomic differences in mortality. Material conditions during one's upbringing, dietary habits, income, housing and working conditions, and psychosocial factors, such as low control, are considered relevant direct or intermediate variables (Bolton Smith et al., 1991; Connor, 1979; Hassan, 1989; Lundberg, 1991; Mackenbach, 1992; Marmot et al., 1984; Mezentseva \& Rimachevskaya, 1992; Olsen \& Kristensen, 1991; Ranchor et al., 1990; Siegrist, 1989; Ultee el al., 1992; Wilkinson, 1990). We shall restrict the discussion to those factors that were directly or indirectly measured in the present study.

Results in Kaunas suggested that the SES of parents could have some direct effects on mortality of their children during adulthood. The high intergenerational stability regarding SES may imply that it is not one's own SES that causes individuals to have differential risks of mortality during adulthood, but the socioeconomic and material conditions during one's upbringing. This might be indicated by the SES of the parents. This background SES could, however, not explain the socioeconomic differences in mortality among KRIS middle-aged men. Hence, KRIS results do not corroborate studies suggesting that adult socioeconomic differences in health are based on persisting differences from childhood (Forsdahl, 1977; Notkola et al., 1985; Ostberg \& Vagerö, 1991).

The SES gradient may reflect differential knowledge of healthy lifestyles. This explanation was supported by the lower prevalence of smoking in the highest occupational strata. However, a high cholesterol level and body mass index were more frequently found among higher occupational groups. Furthermore, the occupational level remained predictive of all-cause mortality even after adjusting for educational level. Therefore, the data do not give strong support to the theory that access to and availability of information explains the SES gradient. Knowledge of a healthful lifestyle is far from similar to healthful behavior.

The higher mortality among lower social classes may be due to health-related social mobility (West, 1991). According to this theory, disease causes individuals to be downwardly mobile, while healthy individuals have better chances of being upwardly mobile. This explanation is supported by the finding that upwardly mobile men were taller. Height to some extent reflects differences in diseases experienced during childhood. However, adjusting for height did not change the relative risks of mortality. Furthermore, an analysis of social mobility among those with CHD-atscreening showed a slight tendency for this group to be characterized by subjects who were either downwardly or upwardly mobile $(P=0.24)$.

Low occupational status may influence survival after a serious disease (Leon \& Wilkinson, 1989). This hypothesis was extensively studied in the Dutch cohort by investigating the factors that influenced the prognosis among those with CHD-atscreening. In this analysis no indications that SES influenced the prognosis after MI were observed (Bosma et al., 1992). 
The KRIS results also show that the educational level of wives independently affected their husbands' mortality risk. Irrespective of their educational level, men whose wives had a low educational level had an increased risk of dying or suffering a nonfatal MI during follow-up. This association was less apparent for CHD mortality and MI incidence in Kaunas. This effect was independent of status inconsistency and SES of the wife's parents, and of the husband's characteristics regarding biological and lifestyle cardiovascular risk factors. The effect of the wife's educational level was also equally predictive of mortality and incidence among men with type $\mathrm{A}$ and type B behavior.

The results do not corroborate most of the studies discussed in the introduction. These studies report that a highly educated wife had adverse effects on her husband's health status. In agreement with our results, only Strogatz et al. (1988) find that men whose wives had a low educational level were at an increased risk of heart disease. Having a partly younger and more recent population than the other studies mentioned in the introduction, they point to possible "secular changes in the social meaning and implications of wife's education". Haynes et al. (1983) also find adverse effects for men married to highly educated wives. They conclude that their results are applicable only to "marriages that were well established before the general acceptance of and increasing influx of women into the work force" and gains made in educational training. Updated situations, they thought, might well shed a different light on the effects of the wife's SES.

According to this hypothesis, being married to highly educated wives should have affected health adversely for two reasons. They are: the small participation in the labor force of Dutch wives in the 1970s and the fact that they also had a much lower educational level than their husbands. The present results, however, suggest that the negative effects of being married to a highly educated wife probably are restricted not only in time, but also in place or culture. Liberal and progressive notions about wives' opportunities regarding their educational and occupational capacities and aspirations may have been more prevalent in the two European populations in the KRIS.

The present results give prominence to wives with a low educational level. Specific interacting dynamics in the marital context, thus, engender heightened cardiovascular responses in their husbands. Smoking being more common among men whose wives had a low educational level may be one possible pathway leading to excess mortality risks. These results agree with results from sociological investigations showing that a variety of a husband's characteristics are affected by his wife's SES (Bakker, 1992; Bonney, 1988; Dirven et al., 1991). Which specific factors have contributed to these differential mortality risks are yet unclear. Dietary habits, use of health care services, and recreational activities all may be more strongly affected by the wife's educational level. It is especially worth investigating whether marriages in which wives are highly educated are characterized by less marital dissatisfaction and disagreements. Furthermore, since KRIS men were increasingly confronted with the "empty nest" phenomenon and were close to retirement, highly educated wives may have contributed to their husbands being better able to cope in the face of such "lifeevents". Finally, as was the case with the men's own parents, the SES of the parents- 
in-law had some direct effects on their risk of mortality. The relative contribution of social and economic resources available to parents and parents-in-law from higher social classes should be investigated further because they actually may increase a person's well-being and health.

Although some effects were not statistically significant at the 0.05 level, the cross-cultural similarity and the size of the relative risks reduces the probability that chance alone is responsible for these results. Since about $50 \%$ of the spouses had a similar education, sparse tables may have partly caused the fact that results sometimes did not reach statistical significance. Problems of multicollinearity were, however, not encountered during the estimation of the models.

In conclusion, we may say that men whose wives had a low educational level had an excess mortality risk, independently of their own educational level. The marital context emerges as a situational factor in which specific marital interactions combine to produce heightened mortality risks for men. The risk of premature mortality is probably not restricted to the wife's educational level. As Carmelli et al. (1985) remark, "an entire constellation of marital dynamics and interplay" may contribute to differences in mortality among spouses. Several authors identify a number of influences as contributing to the variance of a husband's risk factors or mortality (Carmelli et al., 1985; 1989; Eaker et al., 1983; Haynes et al., 1983). One category of these influences consists of specific spousal personality characteristics such as type A behavior, emotional instability, and depression. Some aspects of the marital environment, including spousal social support and marital dissatisfaction, form a second category. A third category comprises some aspects of the nonmarital environment, for example, the workload and a lack support from the boss. Whether similar attributes of men affect their wives' health status must await future studies. For the time being, the results strongly suggest that the scope of studies regarding an individual's risk of mortality could be expanded by including characteristics of significant others. This may be particularly so for the examination of socioeconomic differences in health, in which it is important to include the SES of spouses as well.

Finally, the KRIS data also gives support to the belief that the gradient between SES and CHD changes over time (Antonovsky, 1968; Kawachi et al., 1991; Kunst \& Mackenbach, 1990; Marmot \& McDowall, 1986; Marmot et al., 1978; Morgenstern, 1980; Vagerö \& Norell, 1989). In the group of fathers of KRIS participants, particularly university graduates and those with nonmanual occupations were found to have had coronary events. This was especially so in Kaunas for fathers who were workers, or who had attended elementary school only. They had a risk of coronary events that was more than $80 \%$ lower than that of fathers who were highly educated or who were employees. Among their sons this negative association practically disappeared in Kaunas, while it even reversed in Rotterdam. It should be repeated that the data about parental heart disease are based upon the reports of the participants only. Without doubt this raises questions about the validity of these data. However, the intergenerational differences between the gradients observed in both cohorts were so large that they may be helpful in explaining why the gradients relating SES and 
CHD were relatively flat in both cohorts. If the direction of a gradient changes with time, it necessarily passes a period in which it is absent. The cardiovascular epidemic started in the upper social classes. When it reached its apex, all classes were affected equally. Careful studies by Hoogendoorn (1990) show that in The Netherlands the cardiovascular epidemic reached its apex in the early 1970s. It seems that the KRIS study covered the period during which the gradient started to become negative.

\section{Social Network}

Being nonmarried was found to increase the risk of all-cause mortality and mortality due to CVD and CHD in both cohorts independently of the biological and lifestyle risk factors. In Kaunas higher mortality risks were observed among the widowers and the divorced and in Rotterdam among the singles. No association (and even a tendency toward a negative association) was observed regarding nonfatal MI. The quantity and quality of the social relations with friends and acquaintances were not associated with health outcomes.

The results are in line with the often reported beneficial effect of being married on health (Goldberg \& Novack, 1992; Gove, 1973; Kraus \& Lilienfeld, 1959; Lynch, 1985; Mendes de Leon et al., 1992; Ortmeyer, 1974; Rosengren et al., 1988). They probably underestimate the effect of widowhood because mortality risks of the widowed could not be examined during the initial period of bereavement in which most of the excess mortality occurs (Bowling, 1987; Jones, 1987; Parkes et al., 1969; Stroebe \& Stroebe, 1987).

We have no explanation for the finding that the mortality risk differed between Kaunas and Rotterdam for specific subgroups among the nonmarried. Perhaps the data are influenced by the relatively small numbers among the subgroups of the nonmarried. Possibly the high relative risk of the divorced in Kaunas might be explained by the relative rarity of divorce in Lithuania ( $2.7 \%$ as compared to $4.2 \%$ in Rotterdam), where divorce was strongly condemned by the Roman Catholic church.

The absence of an association of marital status with nonfatal MI corroborates the results of Vogt et al. (1992), who find that the effects on nonfatal CHD were much smaller than the considerable effects on CHD mortality. These authors also find that the effects on mortality were strong after chronic CHD had developed. The KRIS results also show strong effects on CHD mortality among those with CHD-at-screening (Bosma et al., 1992). Some part of the effect on mortality may, thus, be explained by differences in treatment or compliance and attendance to medical regimens among marital status groups. Spouses may significantly contribute to monitoring and controlling their husbands' health behaviors (Umberson, 1992). The importance of social support for individuals with CHD corroborates studies from several other investigators as well (Blumenthal et al., 1989; Hedblad et al., 1992; Ruberman et al., 1984; Williams et al., 1992).

The quantity and the quality of the social network were not associated with any of the endpoints. However, as an index of social support, the assessment was less 
satisfactory. We should, nevertheless, mention that, despite this imperfect assessment, additional analysis suggested that men who were divorced or widowed benefitted from a close network of intimate friends and acquaintances. This is in close accordance with the study of Silverstein \& Bengtson (1991) who specifically examined health-protecting effects of affectionate solidarity in parent-child relationships for widowers.

In conclusion, we may say that being nonmarried increases the risk of mortality, although this risk might be restricted to specific groups among the nonmarried. Further research should examine particularly the extent to which social support from friends buffers the characteristics of being or becoming nonmarried that are detrimental to health. Although never statistically significant, the results of the KRIS suggest that divorced or widowed men might benefit from a close network of intimate friends and acquaintances. Social support may enhance the individual's capacity to survive longer in the face of never having had a spouse or in the face of the loss of a spouse by divorce of death.

\section{Geographic Mobility}

Because nearly all the Lithuanian participants were born outside Kaunas, analysis had to be restricted to the level of urbanization of the childhood home. Kaunas men born in small towns were found to be at increased risk of MI. Rotterdam men born in villages were at increased risk of CVD and CHD mortality. Analysis restricted to the Dutch cohort shows that the risk of all-cause mortality was increased by $54 \%$; the risk of CVD mortality, by $94 \%$; and the risk of CHD mortality, by $131 \%$ for those who were born in villages compared to those who were born in Rotterdam. These increases were independent of the classic risk factors and of the occupational status. Detailed analysis shows that the risk of CVD, especially of nonfatal MI, tended to decrease as the length of residence in Rotterdam increased.

The results strongly suggest that geographic mobility may give rise to adaptation processes that increase cardiovascular risk. Individuals who must become accustomed to behaviors or expectations that are at variance with those from their background run an increased risk. The longer one has had to adapt to city life, the lower the risk becomes.

It should, however, be mentioned that not only stressful mobility effects may be involved, but also direct effects of unknown factors acting at different stages in life on differences in CHD risk in middle-aged men. In other words, factors related to the urbanization level of the childhood home may have independent effects on adult CHD risks, as do factors related to the urbanization level of the place where one lived as an adult. Since the urbanization level in the KRIS did not vary in 1972-1974 (all lived in Rotterdam or Kaunas), it was not possible to distinguish any direct effects of the urbanization level of the childhood home from mobility effects. 


\section{Type A Behavior Pattern, Stress, and Exhaustion}

Type A behavior, as assessed by the short $\mathrm{N}$ version of the JAS, appeared not to be associated with the cardiovascular endpoints. Because nonresponders did not differ from responders in risk factor levels or incidence and mortality risks, this lack of predictive power probably cannot be attributed to nonresponse. It is also rather unlikely that other forms of the JAS would have shown different results. Form B of the JAS and a Dutch adaptation were not predictive of heart disease in the Dutch cohort either (Appels et al., 1987).

Although these data do not give any support to the type A theory, one may not conclude that this theory is false. The assessment of the type A behavior by a questionnaire is less valid than the assessment by the structured interview (Falger, 1989; Haynes and Matthews, 1988; Matthews \& Haynes, 1986). Longer follow-up periods may have led to other conclusions, as in Kaunas the JAS was found to be associated with future MI during the 15-year follow-up period. The mean score on the type A scale of 105 new cases was 2.57 (SD 5.48) and of 210 matched controls, who did not suffer from CHD during follow-up, was 1.33 (SD 5.38) $(P<0.05)$ (Gostautas: personal communication).

Stress, as measured on the Reeder stress scale, was not a cardiovascular risk indicator either. Unfortunately, the Kaunas data were invalidated by a response set. Therefore this conclusion should be restricted to the Dutch part of the KRIS. The Reeder stress scale dates back some 25 years. At that time it was still assumed that stress is a one-dimensional concept. The questionnaire does not differentiate between stable personality traits (item 1) and problems at home (item 4). The questions are rather aspecific and reflect a vague general tendency toward complaint or neuroticism.

One item of the Reeder stress scale (being exhausted mentally and physically at the end of the day) was found to be predictive of CHD mortality occurring during the first years of follow-up. Adjusting for all biological and lifestyle risk factors, the relative risks for exhaustion were $8.68,5.81,3.89$, and 2.60 for the first, second, third, and fourth year of follow-up, respectively. This finding corresponds with the results of other prospective studies, which show that feelings of "vital exhaustion" precede the onset of heart disease (Appels \& Mulder, 1988). The origin of these feelings might be formed by a deterioration of the myocardium or the coronary vessels or by a chronic, unsuccessful struggle to cope with stressful life events or to maintain control over adverse situations. These explanations are not necessarily mutually exclusive. Most likely, deteriorating health and the inability to overcome problems interact in producing a vicious spiral leading to CHD. This is documented in a study by Kop among percutaneous transluminal coronary angioplasty patients. It was found that successful coronary angioplasty resulted in a significant decrease of feelings of exhaustion. At the same time, feelings of vital exhaustion after successful percutaneous transluminal coronary angioplasty were found to increase the risk of a new coronary event within 1 year. (All relevant cardiological variables assessed at angiography were taken into account.) (Kop, 1994). 


\section{Self-rated Health}

The results of the analyses replicated and extended the findings of previous studies, which demonstrated that self-evaluated general health is a predictor of mortality even when the prior physical health status of the participant is taken into account. Especially those who think of their own health as being worse than that of other men of their age do not live as long as those who assess their health as similar or better. This observation is consistent across cultures. It cannot be explained by the traditional risk factors, by knowledge of parental longevity, or by disadvantageous living conditions, such as being nonmarried or less well educated.

The major question raised by these findings is whether a negative evaluation of one's health reflects manifest disease or a prodromal condition of which the participant is diffusely but not specifically aware. We took the influence of manifest heart disease into account statistically by excluding the group with manifest heart disease and by the construction of an index of "severity of heart disease". The last index was rather imperfect because it did not include clinically relevant characteristics such as chronic heart failure. However, we conclude that the reported associations were not confounded by manifest heart disease, because the results were almost similar in the three types of analysis. We took the presence of subclinical disease into account by checking the time dependency of the relative risks and by taking "worrying" into account. The interaction term between follow-up time and self-rated health was never statistically significant. The worry factor did not affect the associations of mortality with Factor I and the two direct questions. Therefore, we conclude that the associations are not confounded by subclinical disease.

It appears that the results were not biased by the large number of men among the screened participants who did not answer the questions. They did not differ from the others regarding mortality risks, incidence risks, biological risk factors and lifestyle risk factors. However, the relative risks may have been underestimated by nonparticipation in the KRIS screening examinations. This nonparticipation was rather considerable in Kaunas, where only $69 \%$ of the total sample (from the baseline survey and the screening) participated in the screening, as compared to $87 \%$ of the Rotterdam sample. In regard to baseline questions, no substantial differences were found between participants of both the screening and the baseline survey and participants of the baseline survey only. A more detailed substudy of the Dutch nonparticipants shows that more of them believed that examinations just make one worry and that most health problems solve themselves (Section 2.3). According to their scores on the Rotter Internal-External Control Scale, the nonparticipants were more likely to think that fate is responsible for their health rather than their own actions and efforts. A substudy of the Lithuanian nonparticipants showed that pathological conditions (hypertension, previous MI, hypercholesterolemia, or impaired glucose tolerance) occurred twice as often among the nonparticipants as among the participants. We note that a remarkable proportion of the nonparticipating Lithuanians had a negative attitude towards medical personnel and examinations (Glasunov et al., 1981). Nonparticipation is also associated with mortality. In Kaunas mortality within a follow-up period of 4.5 
years was 2.5 times higher among the nonparticipants than among the participants (Domarkienne et al., 1987). Nonparticipants in Rotterdam were also found to have an increased mortality risk; $18 \%$ of the nonparticipants died during follow-up compared to $10 \%$ of the participants. For these reasons it is rather likely that nonparticipation resulted in an underestimation of the risks of a negative perception of one's own health.

The item "how would you assess your own health condition?" was not associated with mortality in Kaunas. In contrast Factor II was associated with mortality in Kaunas only. These cross-cultural differences are hard to explain. Given the large difference between Kaunas and Rotterdam in the percentage of participants who said that their health was poor ( $63 \%$ and $16 \%$, respectively), it is likely that this item had a different meaning in the two cohorts.

This study extended the results of other studies, especially with the observation that both Lithuanian and Dutch men who believed that their health condition was worse than that of other men of their age did not live as long. The comparative question had the strongest association with mortality among subjects free of CHD-atscreening. It was even more strongly associated with mortality among those with CHD-at-screening, indicating that this factor may have some clinical relevance too.

Social psychology may give some new suggestions for the explanation and future investigations of the association of self-perceived health and mortality. In social psychology it is argued that those who tend to deprecate or downgrade themselves have a general negative self-image and a diminished sense of mastery. This interpretation was confirmed by the positive correlations of the evaluative factor and the direct questions with the selected baseline and semantic differential test items. Those who rated their health as poor were inclined to think that they could do little to prevent disease. Two of the four items selected to test the hypothesis that a low sense of mastery was associated with a poor perception of health were predictive of mortality.

The concept of a diminished sense of mastery comes close to the concept of a pessimistic explanatory style, a construct developed within the learned helplessness research tradition. Explanatory style is people's habitual manner of explaining the causes of events. Explanatory style affects the extent of helplessness following uncontrollability and affects coping in the broadest sense. This personality characteristic may influence physical well-being because a person who has a fatalistic view does not see the link between behavior and outcome (Peterson \& Seligman, 1987). A fatalistic view may form part of a low sense of mastery and may explain why men who said at baseline that health is generally a matter of luck had an increased mortality risk.

The KRIS data show that an explanation of the findings along the lines of a low sense of mastery is attractive. However, they do not prove that this hypothesis is right. The database does not include a direct measurement of this construct. Contrary to our expectations the answers to the baseline item "do you think that a healthy man of your age could reduce the likelihood of developing some serious illness by taking preventive measures?" was not associated with mortality. When we adjusted the predictive power 
of the health-perception indices for the selected baseline items, the relative risks did not change. Hence, the results at best suggest that such an approach might be fruitful.

\section{Family History of CVD}

An association of a history of CVD of one parent with the other was found in Kaunas. When a father had experienced a cardiovascular event, the risk that the mother also experienced such an event increased by $71 \%$. Similarities in educational level only marginally contributed to this association. The lifespans of parents were not substantially correlated in both populations. Parental age at death contributed neither to their son's risk of mortality nor to his risk of morbidity during follow-up.

Significant associations of a family history of cardiovascular events with the risk of mortality and nonfatal MI were found. The aggregation among siblings appeared to be independent of the effects of a parental history of CVD. Educational and occupational indicators of parents and KRIS participants contributed only marginally to these similarities. Although blood pressure, cholesterol, glucose tolerance and body mass index were higher among men with a family history of cardiovascular events, these factors could not explain the associations.

The absence of effects of parental death and age at death may be explained by the short follow-up period in the KRIS. In fact, all deaths among KRIS participants were premature ( 70 years of age or less). This restriction in the range of age at death of the participant may have caused the rather small correlation between the lifespans of the parents and their sons. On the other hand, other studies also found small correlations, even when deaths of offspring were not restricted to premature deaths (Brand et al., 1992).

Relatively strong effects on cardiovascular endpoints and especially nonfatal MI, however, came from a family history of CVD. Since a cardiovascular history among first-degree relatives was associated with nonfatal MI and CVD both among younger (45 years old) and older (60 years old) KRIS participants, no indications were found of a particularly elevated risk for younger men. This is not in agreement with studies of Colditz et al. (1991), Schildkraut et al. (1989), and Sholtz et al. (1975). A wider age range among KRIS participants might have shown especially elevated risks due to a family history of CVD among younger individuals. Unfortunately, information about age at the time of the CVD events of siblings and parents was not available. This precluded any additional analysis regarding specifically elevated risks when CVD events among family members occurred at younger ages.

The sibling aggregation was independent of the effect of a parental cardiovascular history in Rotterdam. Strong effects were found not only for morbidity, but also for mortality. No interaction was found between sibling and parental cardiovascular histories. This indicates that sibling aggregation was present even in the circumstance that parents did not have a cardiovascular event. It certainly cannot be denied that genetic mechanisms are involved in the atherosclerotic process leading to CVD. However, the risk of CVD was higher among Lithuanian wives whose husbands had 
such a disease. There was aggregation among siblings, even when their parents did not have a cardiovascular history. This suggests that environmental factors shared among family members may help us understand a part of the reported family aggregation of CVD. Shared environmental factors may include attitudes toward health, dietary habits, recreational activities, and coping styles. As type A behavior has been shown to "run in the family", other psychological phenomena might as well (Lundberg et al., 1990). The contribution of such factors to family aggregation of CVD deserves further scientific attention.

The possible environmental influence of social position was small. Hence, it was probably not similarities in social position that were responsible for family aggregation. However, it is highly likely that lifestyles and environmental factors within social classes were not stable between generations, so that the gradient of the association of SES with CHD changed its direction between generations. In other words, similarities in social position between generations did not guarantee stable environmental factors important in the etiology of CHD. Furthermore, the effect of having siblings with a cardiovascular history did not diminish after their parental social position was taken into account. Thus we conclude that similarities in early environments due to social position during upbringing also did not contribute to the sibling aggregation.

In view of the difficulties in analyzing the family aggregation of polygenic, multifactorial diseases such as CVD, efforts have been directed to determining the genetic and environmental influences on risk factors of CVD, such as the cholesterol level (Burke et al., 1991; Lauer et al., 1991; Prenger et al., 1992; Price et al., 1989; Williams et al., 1991, $1990_{\mathrm{a}}, 1990_{\mathrm{b}}$; Williamson \& Kessling, 1990). The extent to which these risk factors are genetically or environmentally determined is not clear yet. There is no consensus regarding the relative role of hypertension, hypercholesterolemia, and impaired glucose tolerance in the explanation of family aggregation (ten Kate et al., 1982; Rissanen \& Nikkilä, 1979; Schildkraut et al., 1989; Snowden et al., 1982). The aggregation of CVD within families could not be explained by important risk factors, such as the cholesterol level. The KRIS results strongly suggest that other causal pathways should be examined, since these factors probably contribute to family aggregation of CVD only marginally. It remains, however, difficult to disentangle genetic and environmental influences.

Since the data of family history of CVD were obtained from self-reports, misclassification exists. Differential misclassification is, however, unlikely, because the family history was ascertained before the onset of diseases in the groups to be analyzed, i.e., men with CHD-at-screening were excluded from the analysis. The less than adequate information about the ages at which first-degree relatives had suffered their cardiovascular events may have resulted in underestimates of the relative risks of a family history of CVD. The high number of missing answers to the questions regarding a cardiovascular family history, especially in Kaunas, probably did not bias the results. Men who gave incomplete data on any of the family history variables did not differ significantly from others regarding mortality and morbidity during followup. 
In conclusion, we may say that in order to identify persons at high risk of CVD it is important to obtain information about CVD among all first-degree relatives, i.e., not only parents. Genes, habits, and customs transmitted to the next generation, shared, and modified by siblings are important factors in family aggregation of CVD. More research into these family processes may prove fruitful in explaining the family aggregation of CVD. 
Chapter 8

\section{DIFFERENCES BETWEEN KAUNAS AND ROTTERDAM}

\subsection{Differences in Mortality and Nonfatal MI}

The Kaplan-Meier product-limit estimate of the survival curves of Rotterdam and Kaunas is shown in Figure 8.1 (life tables for the Dutch and Lithuanian cohorts are presented in Appendix VII). As the life tables and Figure 8.1 show, the probability of dying during the follow-up was smaller in Rotterdam. The logrank test shows significantly lower survival rates for the Kaunas men. The probability of dying of CHD was somewhat higher in Rotterdam. No differences were found in mortality due to all CVD.

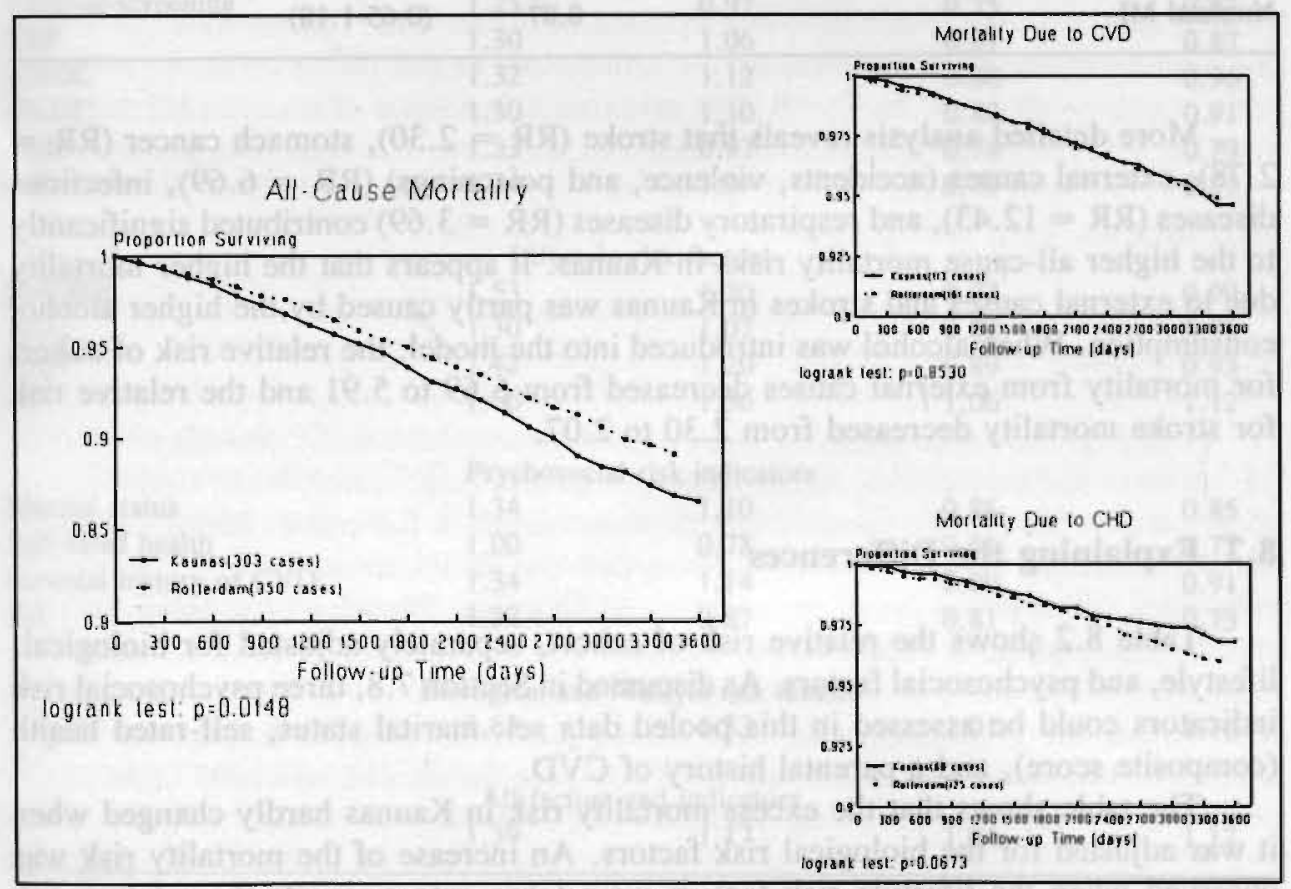

Figure 8.1 Kaplan-Meier curves for Kaunas and Rotterdam.

To compute the risks associated with being Lithuanian or Dutch, the cohorts were pooled and a variable for cohort ( 0 for Rotterdam and 1 for Kaunas) was introduced. Table 8.1 presents the age-adjusted relative risks, showing the risk for Lithuanians compared to the risk for the Dutch. The Kaunasians appeared to have a $30 \%$ elevated 
mortality risk compared to Rotterdammers, whereas CHD mortality risks were $20 \%$ lower in Kaunas.

The cumulative incidence of nonfatal MI was $4.3 \%$ in Rotterdam and $3.6 \%$ in Kaunas. The follow-up periods of the cohorts were equalized by restricting them to 8 years, as this was the shortest follow-up period of any of those who had been screened and survived throughout follow-up without having a nonfatal MI. Adjusting for age in a logistic regression analysis resulted in a risk of MI that was $13 \%$ smaller in Kaunas than in Rotterdam.

Table 8.1 Age-adjusted relative risks indicating the mortality risk of men in Kaunas compared to the risk of Rotterdam men.

\begin{tabular}{lrr}
\hline & RR & $95 \%$ Cl \\
All causes & 1.30 & $(1.12-1.52)$ \\
CVD mortality & 1.05 & $(0.82-1.34)$ \\
CHD mortality & 0.81 & $(0.60-1.08)$ \\
Nonfatal MI & 0.87 & $(0.65-1.18)$ \\
\hline
\end{tabular}

More detailed analysis reveals that stroke $(R R=2.30)$, stomach cancer $(R R=$ 2.78), external causes (accidents, violence, and poisonings) $(R R=6.69)$, infectious diseases $(R R=12.43)$, and respiratory diseases $(R R=3.69)$ contributed significantly to the higher all-cause mortality risks in Kaunas. It appears that the higher mortality due to external causes and strokes in Kaunas was partly caused by the higher alcohol consumption. When alcohol was introduced into the model, the relative risk of cohort for mortality from external causes decreased from 6.69 to 5.91 and the relative risk for stroke mortality decreased from 2.30 to 2.07 .

\subsection{Explaining the Differences}

Table 8.2 shows the relative risk of cohort, separately adjusted for biological, lifestyle, and psychosocial factors. As discussed in Section 7.8, three psychosocial risk indicators could be assessed in this pooled data set: marital status, self-rated health (composite score), and a parental history of CVD.

The table shows that the excess mortality risk in Kaunas hardly changed when it was adjusted for the biological risk factors. An increase of the mortality risk was observed when the lifestyle risk factors were taken into account; the relative risk changed from 1.30 to 1.66. This means that if the lifestyles of the Lithuanians had been as disadvantageous as those in Rotterdam, the difference in mortality between the cohorts would even have been larger. For example, the relative risks of all-cause mortality in Kaunas would have increased from 1.30 to 1.53 if the Lithuanians had smoked as much as their Dutch counterparts. This means that one or more factors not 
commonly asked about in routine medical examinations accounted for the differences in life expectancy between Kaunas and Rotterdam.

One of these factors was self-rated poor health. Adjusting for this factor reduced the difference to zero, so that if the Lithuanians had been as optimistic about their health as the Dutch, they would have had the same life expectancy (within the defined age range).

Table 8.2 Age-adjusted relative risks of nationality [cohort: 0 (Rotterdam) and 1 (Kaunas)], separately adjusted for risk factors and indicators.

\begin{tabular}{l|l|l|l|l}
$\mathrm{AGE}$ & 1.30 & 1.05 & 0.81 & 0.87
\end{tabular}

Adjusted for age and:

$\begin{array}{lllll}\text { CHD-at-screening } & 1.25 & 0.97 & 0.75 & 0.82 \\ \text { SBP } & 1.30 & 1.06 & 0.81 & 0.87 \\ \text { CHOL } & 1.32 & 1.12 & 0.88 & 0.96 \\ \text { GLUC } & 1.30 & 1.10 & 0.82 & 0.91 \\ \text { BMI } & 1.33 & 0.97 & 0.74 & 0.79 \\ \text { All } & 1.34 & 1.07 & 0.79 & 0.91\end{array}$

$\begin{array}{lllll}\text { SMOKE } & 1.53 & 1.20 & 0.97 & 0.99 \\ \text { ALC } & 1.30 & 1.07 & 0.82 & 0.92 \\ \text { PHA } & 1.42 & 1.20 & 0.89 & 0.93 \\ \text { All } & 1.66 & 1.36 & 1.06 & 1.12\end{array}$

Marital status

Self-rated health

Parental history of CVD

All

\section{All-cause mortality CVD mortality CHD mortality Nonfatal MI*}

1.05

0.81

Adjusted for:

\begin{tabular}{lllll} 
& \multicolumn{3}{l}{ Psychosocial risk indicators } & \\
Marital status & 1.34 & 1.10 & 0.86 & 0.85 \\
Self-rated health & 1.00 & 0.78 & 0.61 & 0.77 \\
Parental history of CVD & 1.34 & 1.14 & 0.89 & 0.91 \\
All & 1.22 & 0.87 & 0.81 & 0.73
\end{tabular}

Biological and lifestyle risk factors

$\begin{array}{llll}1.63 & 1.33 & 1.01 & 1.16\end{array}$

All factors and indicators

$\begin{array}{llll}1.39 & 1.17 & 1.04 & 1.12\end{array}$

a Nonfatal MI was analyzed using logistic regression analysis with an eight-year follow-up period.

No meaningful differences were observed concerning the risk of mortality due to CVD. The age-adjusted risk hardly changed when the biological risk factors were taken into account. It increased with adjustment for the lifestyle risk factors, which means that CVD mortality would have increased if the Lithuanians had smoked as 
much and exercised as little as the Dutch. If the Lithuanians had had the same optimism about their health, the mortality due to CVD would have been lower in Kaunas than in Rotterdam.

Being Lithuanian reduced the risk of CHD mortality by approximately $20 \%$ (RR $=0.81$ ). This difference could not be explained by the biological risk factors; the CHD mortality risk in Kaunas would have been somewhat higher if the Lithuanians had had the same body mass index. The lower CHD mortality was due to the lower prevalence of unhealthy lifestyles and would have been even lower if self-rated health had had the same values as it had in Rotterdam.

Being Lithuanian reduced the risk of nonfatal MI by $13 \%(R R=0.87)$. The difference was due to the more favorable levels of cholesterol and smoking in Kaunas and would have been even larger if self-rated health had been less negative.

The multivariate model was also analyzed with all risk factors and indicators simultaneously. The results show that the risks for the Kaunas population would have been greater than those of the Rotterdam population if the Lithuanians had had equal levels of all risk factors.

The worse prognosis for the Lithuanians, in the case that they had had a risk profile similar to the Dutch one, is illustrated in Figure 8.2. The survival curves for all three endpoints become worse for the Lithuanians as risk factor levels are assumed to be similar to those of the Dutch. The cumulative incidence of nonfatal MI per 1000 men in 8 years would be 30.1 instead of 23.8 . In other words, the health of the Lithuanian cohort was, in general, protected by a more favorable lifestyle, and was only impaired by a poor perception of their own health.

\subsection{Summary and Discussion}

Although all-cause mortality risks were higher in Lithuania, mortality risks due to "affluent and modern" diseases, such as CHD and lung cancer, were higher in The Netherlands. Most other causes of death were higher in Lithuania. Although rare in both populations (nine deaths in Kaunas and one in Rotterdam), mortality due to infectious diseases was much more common in Kaunas. The high Lithuanian risk was almost entirely due to tuberculosis. Stomach cancer mortality risks in Kaunas were also higher (18 and 8, respectively). Dietary habits, such as relatively low intake of fresh fruits and vegetables, may have been responsible (Zaridze \& Basieva, 1990). Higher alcohol consumption was partly responsible for the elevated risks of mortality due to stroke ( 22 in Kaunas and 15 in Rotterdam). Finally, the excess mortality risks due to respiratory diseases (12 and 5 , respectively) may be linked to housing and environmental conditions.

The much higher mortality risks due to accidents, violence, and poisoning (48 and 10 respectively) merit some further attention. Unfortunately, no distinctions could be made among these external causes of death. Kaunas medical personnel believed that suicide and violence formed the largest part of this category. High risks due to this cause of death in the former Soviet Union are frequently reported (Deev \& Oganov, 


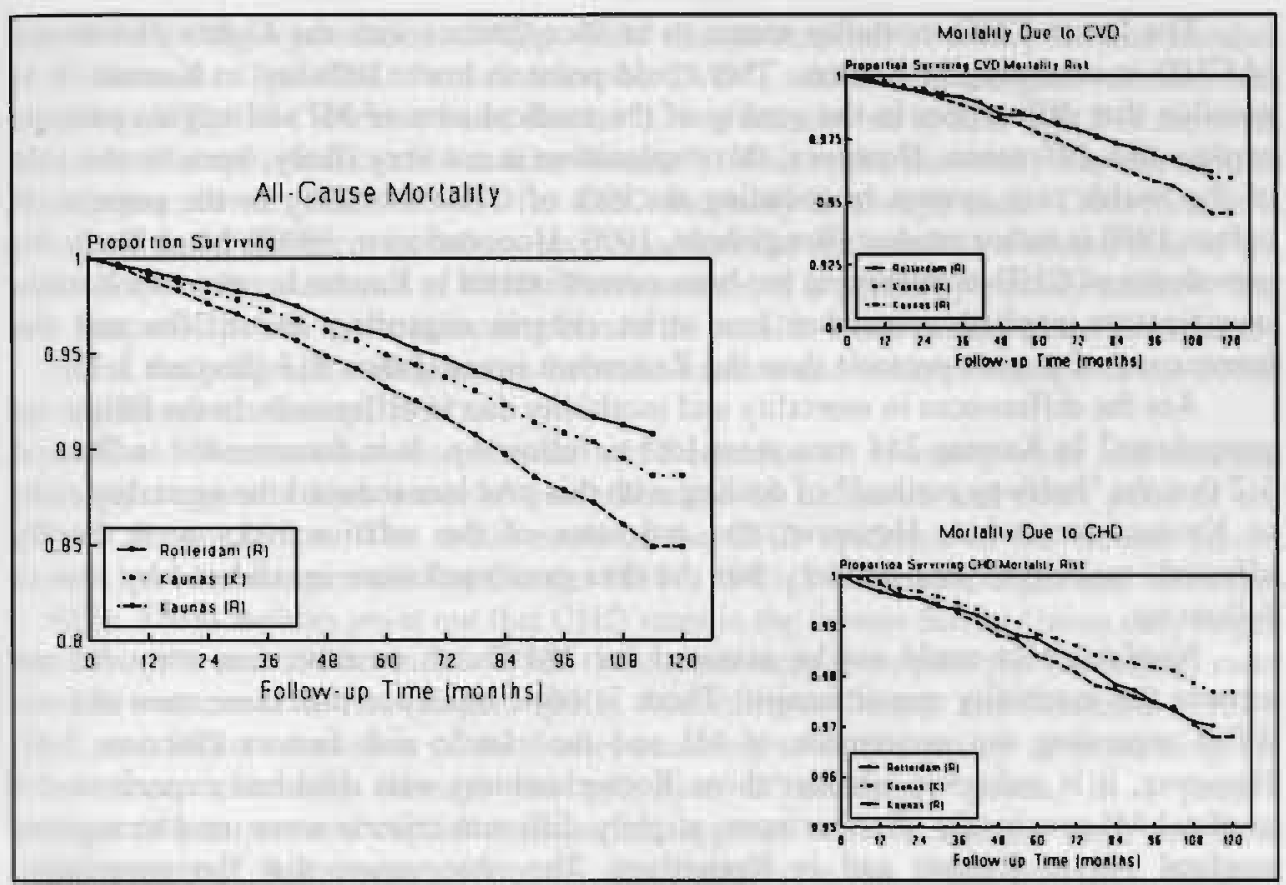

Figure 8.2 Survival curves assuming similar levels of risk factors [curves are estimated with mean values of all covariates of the population (in brackets)].

1989; Garbe \& Garbe, 1990; Mezentseva \& Rimachevskaya, 1990; Virganskaya \& Dmitriev, 1992). Not only was the excess mortality caused by cerebrovascular diseases and mental disorders (i.e., alcoholism) associated with the higher alcohol consumption in Kaunas, but so was the excess mortality due to external causes.

The lower risks of CHD mortality and nonfatal MI in Kaunas could be explained to a large extent by lifestyle factors, especially smoking. The lower mean value of the cholesterol levels and the higher percentage of married men also contributed to the smaller CHD risk in Kaunas, although to a smaller degree. Consequently, the higher risk of all-cause mortality and the somewhat increased risk of CVD mortality (stroke in particular) would have been even higher if the Kaunas population had had the same risk profile as the Rotterdam population. The differences between Kaunas and Rotterdam concerning CVD mortality, CHD mortality, and nonfatal MI were suppressed to a large extent by differences in health perception. A negative evaluation of one's health was associated with mortality in both cohorts, and the Kaunas population rated their health as poor much more often than the Rotterdam population. This psychological factor explained most of the differences in all-cause mortality. If the Kaunas population had been as optimistic about their health as the Dutch population, they would have had approximately the same mortality risks, while the difference in CHD mortality would have been $40 \%$. 
The lower CHD mortality seems to be incongruous with the higher prevalence of CHD-at-screening in Kaunas. This could point to lower lethality in Kaunas. It is possible that differences in the quality of the medical care of MI and angina patients explain this difference. However, this explanation is not very likely, because the role of the health care system in reducing the risk of CHD mortality in the population before 1980 is rather modest (Beaglehole, 1990; Hoogendoorn, 1990). Most likely the prevalence of CHD-at-screening has been overestimated in Kaunas because the Kaunas investigators applied somewhat less strict criteria regarding the ECGs and the assessment of angina pectoris than the Rotterdam investigators did (Section 3.1).

Are the differences in mortality and morbidity due to differences in the follow-up procedures? In Kaunas 244 men were lost to follow-up. It is documented in Section 5.2 that the "halfway method" of dealing with this problem reduced the mortality risks in Kaunas somewhat. However, the estimates of the relative risks were hardly affected, making it very unlikely that the data presented were invalidated by loss to follow-up.

Nonfatal MIs could not be assessed for 284 Dutch men because they did not answer the morbidity questionnaire. There is some evidence that these men did not differ regarding the occurrence of MI and the classic risk factors (Section 2.4). However, it is unknown whether those Rotterdammers who died had experienced a nonfatal MI previously. Furthermore, slightly different criteria were used to register nonfatal MI in Kaunas and in Rotterdam. The observation that the cumulative incidence of nonfatal MI was higher in Rotterdam should, therefore, be interpreted cautiously, although two factors point to a higher incidence in Rotterdam. The absolute number in Rotterdam may have been underestimated by defining the group with "possible" MI as free of MI and by the fact that this number did not include those who suffered a nonfatal MI before their death.

Certification of the underlying cause of death is not free of well-documented problems (Ruzicka \& Lopez, 1990; Uemura \& Pisa, 1988; World Health Organization, $1977_{\mathrm{b}}$ ). The validity of mortality statistics is primarily a reflection of autopsy rates, diagnostic techniques, the medical training of physicians, and the extent to which deaths have been certified medically. The autopsy rate in Kaunas $(53 \%)$ was much higher than in Rotterdam (less than 10\%). This is reflected in the much smaller number of deaths due to unknown causes and ill-defined conditions in Kaunas (13 deaths in Kaunas and 35 in Rotterdam). It is impossible to quantify the magnitude of possible biases in the coding of causes of death exactly. Therefore, care should be taken in interpreting the results. This is particularly true for specific causes of death, such as CHD. The autopsy rates were relatively low in Rotterdam; CHD may have been erroneously diagnosed in some cases in which death was caused by other heart diseases. However, when mortality due to CHD and other heart diseases was taken together, Kaunas men still had lower risks of mortality due to heart diseases.

Both centers used the ICD for the coding criteria for cause of death categories. In Kaunas the eighth revision of the World Health Organization manual was used throughout the follow-up period (World Health Organization, 1967). This system was used in Rotterdam until January 1, 1979. Thereafter the ninth revision was used 
(World Health Organization, 1977.). This change resulted in a very limited number of differences. For CHD "arteriosclerotic CVD", which in the eighth revision was coded as ICD 412 ("chronic heart disease"), was transferred to ICD 429.2 in the ninth revision ("unspecified cardiovascular disease"). This code, however, was not used in Rotterdam; hence, ICD $410-414$ for CHD mortality is always used. Therefore, it is assumed that differences in follow-up procedures did not result in a severe bias of the difference in the incidence of CHD. Furthermore, it is probably equally unlikely that the nonparticipation in the screening biased the results, since results of the Erica Study confirm the geographic differences between eastern and western Europe in the 1970s (Erica Research Group, 1991).

The differences between Kaunas and Rotterdam in mortality risks and nonfatal MI may suggest a later onset of modernization in Lithuania and other parts of the former Soviet Union. The social roots of the CHD epidemic in the former Soviet Union have been expounded by Cooper $(1981,1983,1987)$ and Cooper and Schatzkin (1982). These authors point out that CHD rates in the former Soviet Union only began to rise in the mid 1960s. In the 1980s these rates were to surpass the (declining) rates in most western countries (Lopez, 1990). According to Cooper and Schatzkin (1982), "the post-Stalin orientation of the Soviet economy toward a policy of motivating the work force primarily through the provision of consumer goods, in a pattern comparable to western capitalist countries, appears to have laid the basis for these developments." A consumer economy developed from the 1950s onwards. Western Europe and the United States had gone through this modernization/industrialization process earlier.

Such differences in the lifetime experiences of individuals probably contributed to the higher CHD risks in Rotterdam in the 1970s. Relevant differences between Kaunas and Rotterdam in lifetime experiences were probably reflected in higher cholesterol and glucose tolerance levels, much less physical activity, and a greater number of smokers in Rotterdam. Moreover, the Dutch reported that they started smoking at much younger ages than their Lithuanian counterparts. The more frequently reported cardiovascular history among parents in The Netherlands may also point to an earlier onset of affluent living habits in Rotterdam. This factor partly protected the Lithuanians from higher CHD mortality and MI incidence risks. Furthermore, Kaunas men were also partly protected from higher CHD mortality risks by a less individualized sociocultural environment, as indicated by a closer network of friends and fewer single men.

In conclusion, we may say that, compared to the Dutch population, the Lithuanian men in the 1970s were still protected from higher mortality and morbidity risks by their cardiovascular risk profile, in which especially smoking was less prevalent. This protective effect was substantially suppressed by factors leading to or resulting from a perception of their own health as poor. In Section 7.9 it is argued that a negative perception of health may reflect a poor sense of mastery, which is usually acquired by experiences of noncontingency between efforts and outcome. This may refer to idiosyncratic experiences but also to society-induced experiences. The high prevalence of a negative health perception in the Lithuanian population may reflect specific 
Chapter 8

sociocultural conditions, which actually may contribute to reported geographic differences in mortality and disease. 


\section{Chapter 9}

\section{SUMMARY}

\subsection{History and Aims of the KRIS Follow-up Study}

In the late 1960 s the World Health Organization initiated a feasibility study on the problems of general methodology of large scale intervention studies of CHD. From the point of view of the future applicability of the results it was considered particularly relevant that the study be conducted by centers located in countries with different health service systems. The Kaunas Medical Institute (Kaunas, formerly Lithuanian SSR) and the Rotterdam Municipal Health Center together with the Erasmus University (Rotterdam, The Netherlands) were selected as the participating centers. Hence the name of the study: Kaunas-Rotterdam Intervention Study (KRIS).

The data collection started in March 1972 and ended in December 1974. In each city a sample of 4000 men between 45 and 59 years of age and representing various socioeconomic strata of an urban population was identified. These men were interviewed at the baseline survey, and invited to participate in a screening examination (Glasunov et al., 1981). In Kaunas $2452(69 \%)$ and in Rotterdam $3365(87 \%)$ of the invited men participated in the screening. At the baseline survey or screening information was collected regarding a large number of biological, lifestyle, and psychosocial risk factors.

Both centers separately collected information about the occurrence of cardiovascular events during a ten-year follow-up. In Kaunas the participants were followed by means of a mortality and MI register covering the whole population of Kaunas. In Rotterdam follow-up data were collected by inspection of death certificates and by a questionnaire sent in March 1982 to all surviving participants. This questionnaire was returned by $91 \%$ of the participants. Discharge letters of cardiologists and archives of general practitioners were used to verify the medical history of those with heart disease. Both centers made use of this database to do some longitudinal investigations of the determinants of CVD or other diseases.

In 1988 both teams decided to cooperate in the analysis of the ten-year follow-up data. The desirability of a common follow-up study originated from some unique properties of the KRIS: the unusual amount of psychological and sociological information collected, the opportunity to cross-validate the predictive power of some supposedly behavioral risk factors in populations living in different socioeconomic conditions and facing an increase (Lithuania) and a decrease (The Netherlands) of CHD incidence. Therefore, the emphasis of the KRIS follow-up study was laid upon socioeconomic and psychological factors. 


\subsection{Prevalence of Risk Factors and Indicators}

At screening some minor and some major differences between the cohorts were observed. Significant differences were observed in mean age (52 and 53 years in Kaunas and Rotterdam, respectively), mean diastolic blood pressure ( 88 and 80 ), mean body mass index (27 and 26), mean cholesterol level (193 and 201), mean glucose tolerance (146 and 165), smoking (subjects who "never-smoked": $27 \%$ and $8 \%$ ), alcohol consumption (subjects classified as "immoderate": $31 \%$ and $15 \%$ ), mean physical activity (Reiff score 209 and 151) and a positive family history of CVD (parents $32 \%$ and $40 \%$; siblings $4 \%$ and $14 \%$ ). No differences were observed in systolic blood pressure (138).

At screening, information was collected about the educational and occupational level of the participant, his wife, parents and parents-in-law. The level of education and the occupational status were measured differently in each cohort. Therefore, the distributions were hard to compare.

Both cohorts differed in some other social characteristics. Lithuanian men were more often married and had a more extended social network. Almost none of the Lithuanian participants was born in Kaunas, while half the Dutch participants had always lived in Rotterdam.

Kaunas participants showed somewhat more signs of the coronary prone type A behavior on form $\mathrm{N}$ of the JAS. This difference was not significant. Unfortunately enough, it was not possible to compare the cohorts on the Reeder stress scale, because a response set was found to bias the Lithuanian data.

A striking difference between the cohorts was observed in the average perception of their health status. The participants rated their health on a semantic differential test for "my health" and on two direct questions: "How would you assess your own health?" and "What do you think of your own health compared to that of other men of your age?" Factor analysis of the semantic differential test showed that this test measured a general evaluation of health and the extent to which subjects worried about their health. Remarkable differences were observed between the cohorts. More Rotterdammers than Kaunasians considered themselves in good health and they also worried less about their health. Of the participants $16 \%$ in Rotterdam and $63 \%$ in Kaunas rated their health as poor. Thirty-one percent of the Lithuanians thought their health status was worse than that of other men, compared to $14 \%$ in Rotterdam.

\subsection{Incidence of Main Endpoints}

The biological, lifestyle, and psychosocial factors were related to: (1) all-cause mortality, (2) mortality caused by all CVD, including CHD and cerebrovascular disease (ICD code 390-459), (3) mortality due to CHD (ICD code 410-414), (4) nonfatal MI. The number of deaths due to all causes per 10,000 person years was 146.37 in Kaunas and 121.73 in Rotterdam. Corresponding numbers for mortality due to CVD were 54.59 and 55.99. The number of CHD deaths was 32.85 and 43.47, 
respectively. Cumulative incidence of nonfatal $\mathrm{MI}$ in Kaunas was $3.6 \%$ and in Rotterdam $4.3 \%$. This points to a higher incidence of CHD in Rotterdam. Other analyses revealed that the higher all-cause mortality risk in Kaunas was a result of higher risks of mortality due to external causes (accidents, suicide), stomach cancer, cerebrovascular disease, infectious diseases, and respiratory diseases. Modern diseases, such as CHD and lung cancer, were higher in the Dutch part of the KRIS.

\subsection{Impact of Risk Factors and Indicators}

Smoking was found to increase the risk for all endpoints in both cohorts. A high cholesterol level was associated with increased risks of the three cardiovascular endpoints in both cohorts. Elevated systolic blood pressure increased the risk for all endpoints in Kaunas and for all-cause mortality and CVD mortality in Rotterdam. An impaired glucose tolerance was positively associated with all endpoints in both cohorts. However, the strength of the associations did not reach conventional levels of statistical significance for CHD mortality in either cohort nor for all-cause mortality and nonfatal MI in the Dutch cohort. The body mass index was found to increase the risk of CVD and CHD mortality in both cohorts. This effect disappeared after adjusting for blood pressure. Generally, protective effects were found for alcohol consumption. Apart from moderate drinking and all-cause mortality in The Netherlands, these effects were, however, never significant in the multivariate models. As for physical activity, rather small effects were observed. Data about changes in risk factor levels were available for Kaunas only. Changes in blood pressure and cholesterol level contributed to the increased risk of all-cause mortality. A change in diastolic blood pressure was a significant predictor of CHD mortality.

A positive history of CVD of one of the parents was not associated with all-cause mortality or CVD mortality. However, in both cohorts, a parental history of CVD increased the risk of nonfatal MI. Due to small numbers it was impossible to compute the risk due to having a sibling with a positive cardiovascular history in Kaunas. The Dutch data showed that having a sibling who suffered from CVD doubled the risk for all cardiovascular endpoints. Having a sibling with cardiovascular history seems to bear more risk than having a parent who suffered from heart disease. The elevated cholesterol level of those who had a parent and a sibling with a positive history of CVD may suggest genetic influences. Taking the cholesterol level and all other risk factors into account, however, hardly changed the relative risks. Therefore, the data suggest that sharing a family culture and a common environment might be at least as important as sharing genes.

The importance of family culture was also suggested by the analysis of the educational level of spouses. The educational level of the participant himself was not associated with any of the endpoints, except all-cause mortality in Rotterdam. The mortality risk was increased by $50 \%$ among Dutch participants with an intermediate or low educational level. However, the educational level of a participant's wife was significantly associated with his longevity in both cohorts. The excess mortality risk 
of having a wife with a low educational level (age and the educational status of the participant taken into account) was $57 \%$ in Kaunas and $115 \%$ in Rotterdam. The biological and lifestyle risk factors only partially accounted for this excess mortality risk. Therefore, the data strongly suggest that yet unknown cultural factors associated with a wife's educational level influence the health of her husband. Further explorations of the family background suggested that the educational level of the parents-inlaw might be involved in this cultural context as well.

The finding that, in both countries, the wife's educational status has an independent influence upon the longevity of her husband raises a number of questions that deserve further scientific attention. A wife has a strong influence on the lifestyle of a family. We do not know which elements of the total lifestyle are important. Diet and smoking do not account for it completely; the association remained significant when smoking and cholesterol levels were taken into account. Other factors such as general hygiene, use of health care, sleeping habits, and the way in which financial and educational problems are dealt with may be important too. The role of the family, its culture and values, and ways of communication in a family deserve more attention than they actually receive. Studies of coronary patients indicate that they generally experienced more problems at home than at work (Falger, 1989). The observation that having a sibling with a positive history of CVD bears more risk than having a parent with a positive history points in the same direction. Therefore, we recommend encouraging investigations of the influence of the "family culture" upon the health of the family members.

Occupational level was consistently associated with all-cause mortality crossculturally. A strong tendency toward a negative association with the cardiovascular endpoints was observed in Rotterdam. When both education and occupation were included in the same analysis, occupation had the stronger association with all-cause mortality. Adjusting for classic risk factors resulted in a slight decrease of relative risks. In Kaunas lower occupational status was no longer significantly associated with mortality, although the tendency still existed (RR $=1.24 ; 95 \% \mathrm{CI}: 0.96,1.59)$. In Rotterdam the relative risk of all-cause mortality remained significant $(R R=1.63$; $95 \% \mathrm{CI}: 1.07,2.48)$. Status inconsistency and intergenerational mobility had no confounding or modifying effect on the association of SES with mortality. Neither did differences in educational level; the risk of occupation hardly changed when adjusted for education. Hence, socioeconomic differences in mortality were not uncommon in the eastern European part of the KRIS.

The relative absence of an association of SES and CHD (especially in Kaunas) does not correspond with the current literature, which usually reports that the incidence of CHD is elevated in underprivileged groups. This might be because the data were collected during a period in which the gradient between SES and CHD started to change. Several authors have suggested that the SES-CHD gradient may change with time. The rise of the CHD epidemic started in the upper strata and affected all social classes equally at its apex. Thereafter, CHD became more prevalent in the lower strata. If this is true, the gradient necessarily passes a period of zero correlation. 
Can the relative absence of the SES-CHD gradient in the KRIS study be explained by the fact that the data were collected during a period in which the gradient started to change? This was investigated by computing the "occurrence of a coronary event" among fathers and their sons in relation to their SES. Among the fathers of the KRIS participants coronary events were usually found among university graduates and those with nonmanual occupations. This was especially so in Kaunas, where fathers who were workers, or who had attended elementary school only, had a risk of a coronary event that was reduced by more than $80 \%$. In the Kaunas cohort the positive association almost disappeared, but not completely. Lithuanians with a low SES had a slightly smaller risk of CHD than Lithuanians with a high SES. In Rotterdam low SES fathers had a significantly reduced risk (approximately $40 \%$ ) of a coronary event. This gradient reversed in their sons to an increased risk (approximately $60 \%$ ).

The finding that the SES-CHD gradient actually reversed in the Dutch population suggests that the CHD rates were already declining in the upper social classes in the 1970s. The CHD epidemic in the Dutch population had already passed its apex. It is in this context that, compared to the Lithuanian risk profile, the Dutch profile still consisted of more characteristics typical of modern society. Some examples are: a higher rate of divorce, less stable and less extended social networks, less physical activity, a higher percentage of cigarette smokers and a higher mean cholesterol level.

The belief is expressed (in Sections 1.1 and 4.1) that the SES gradient follows a dynamic pattern and changes with time as a function of the rise and fall of the coronary epidemic. This belief acquires credibility from the data, but no proof. The very proof of this model would require the inclusion of more cohorts and more information about risk factors among fathers and about lifestyle changes in time. This type of investigation is firmly recommended, not only because it would give more insight into the socioeconomic, psychological, and demographic origins of the rise and fall of the coronary epidemic, but also because other more or less lifestyle-related diseases, such as lung cancer, may follow a similar pattern.

Two elements possibly involved in the dynamic pattern of the coronary epidemic might be marital status and geographic mobility. In modern society divorce is increasing, and more people choose to live alone. Divorced men had highly elevated risks of CVD and CHD mortality in Kaunas, whereas single men had the excess risk in Rotterdam. It is likely that sociocultural factors accounted for this difference between Kaunas and Rotterdam. Furthermore, there were some indications that a close social network lowered the risks for the widowed and divorced.

Increasing industrialization may also increase the number of people who leave their sociocultural niche. It was impossible to investigate exactly the health effects of geographic mobility in Kaunas, because almost all participants were born outside Kaunas and moved to that city on an involuntary basis. Rotterdam men born in villages were at increased risk of CVD and CHD, independently of their biological and lifestyle risk factors and of their SES. Detailed analysis shows that the cardiovascular risk tended to decrease, as residence in Rotterdam was extended. This finding clearly points out that geographical mobility gives rise to adaptation processes that increase cardiovascular risk. 
Two psychological factors commonly believed to be associated with modernization or industrialization, namely type A behavior and "stress" were not associated with any of the endpoints. This does not refute the type A theory in general. An assessment of type A behavior by questionnaire is less valid than an assessment by interview. The negative finding corresponds with other recently published studies and contributes to the declining attractiveness of the type A theory in general. However, such studies do not prove that the original findings, mainly from the 1960s, were false. Type A behavior possibly exerts its influence mainly during the initial stage of the coronary epidemic, when the initial impact of societal changes causes feelings of loss of control and aggression in unprepared subjects. The slightly elevated mean type A scores of the Lithuanian cohort and the observation by Gostautas (Section 7.9) that type A behavior was predictive of CVD over a longer follow-up period give some support to this belief, but no proof.

Because in Lithuania the replies to the Reeder stress scale were adversely influenced by a response set, the analysis had to be restricted to the Dutch cohort. No associations were observed between the scale scores and any of the endpoints. However, positive results could hardly have been expected because the Reeder stress scale is an outdated instrument. The items of the scale refer to rather different domains. One of them refers to the unusual tiredness that results from prolonged stress ("At the end of the day I am completely exhausted mentally and physically"). This item had good predictive power for CHD mortality occurring during the first years of follow-up. This result fits into the observations made in many retrospective and some prospective studies that a state of exhaustion precedes the onset of CVD (Appels \& Mulder, 1988; Appels \& Schouten, 1991; Kuller, 1978; Ladwig, 1989). Many cardiologists have been inclined to attribute this association to subclinical heart disease. Recent studies by Carney et al. (1988) and by Kop (1994) show that feelings of exhaustion and lack of energy increase the risk of a new coronary event in patients who have received successful angioplasty (taking all relevant cardiological factors observed during angiography into account). Therefore, these studies support a psychological interpretation of the finding that a state of exhaustion precedes CHD.

An important finding of the KRIS follow-up study is the strong and crossculturally consistent effect on health of the belief that one's health is poor. Especially those who believed that their health was worse than the health of their peers were at increased risk of all-cause mortality and CVD mortality in both cohorts and of CHD mortality in Rotterdam. Lithuanians were much more pessimistic about their health. The most striking finding of the KRIS follow-up study is the observation that the $30 \%$ excess mortality of the Lithuanian cohort could be explained by this pessimistic health perception. This factor also suppressed the advantageous position of the Lithuanian cohort as for the cardiovascular endpoints. Had the Lithuanians been as optimistic about their health as the Dutch, the differences in the incidence of CHD mortality and morbidity would have been even larger.

In section 4.6 it is argued that a pessimistic attitude toward health reflects a low sense of mastery. The positive correlations between health perception and those baseline items that express the feeling that one can do something to protect one's 
health support this interpretation. Other interpretations (a negative perception of health reflects subclinical disease, knowledge of parental longevity, or unfavorable socioeconomic conditions) were not supported by the data. These results lead to the recommendation of giving high priority to the investigation of health perception in health psychology and in public health. Health psychology should investigate the elements that do or do not belong to this factor and develop instruments to measure it. Questions that ought to be investigated are: Do comparative questions have more predictive power than self-descriptions (as in this study)? How is this behavior acquired? Does a pessimistic perception of health reflect a negative explanatory style? What are the associations with health behavior, e.g., smoking? As the populations were found to differ considerably in their health perception, public health scientists should investigate the societal origins of a fatalistic perception of health. Questions that ought to be investigated are, for example: Is the health perception in a population associated with characteristics of the health care system? Is a negative perception of health part of a generalized feeling of noncontingency of effort and outcome, which affects other aspects of life as well?

The answer to the question which psychosocial factors influence longevity and increase the risk of CVD mortality can now be summarized as: men from low social classes, men whose wives have a low educational level, nonmarried men and men with self-rated poor health have a reduced life expectancy, independent of their classic risk factors or the culture they live in. Being nonmarried, geographically mobile, having a gloomy outlook on health, and a family history of CVD increase the risk of fatal or nonfatal CHD, independently of the traditional risk factors and the culture in which one lives. Being exhausted increases the risk of CHD as well. However, the crosscultural consistency of this last factor could not be checked because of technical problems.

How much do psychosocial factors contribute to the identification of high-risk subjects? This question is important for designing cost-effective intervention strategies directed at individuals. ROC curves were selected as the statistical method to answer this question. ROC curves provide information about the increase of the discriminatory power of a set of tests when a new test is added. Originally this method was developed for the evaluation of new diagnostic tests in clinical situations. Because of computational problems, which were mainly caused by empty cells, it was not possible to include all the psychosocial factors in one model together with all the other risk factors. Therefore, ROC curves were computed using a limited number of psychosocial factors (self-rated health, marital status, and parental history of CVD). Due to this reduction the computation of the ROC curves became mainly a methodological exercise.

The results show that, when 1 - specificity $=20 \%$ was chosen as an optimal level of specificity, the sensitivity for CHD mortality of age alone was $30 \%$ at this point. Adding the psychosocial factors increased this to $41 \%$. Adding the eight biological and lifestyle risk factors to the model increased the sensitivity to $57 \%$. When all risk factors were included in the model, the sensitivity increased to $61 \%$. 
This analysis should mainly be considered as a methodological exercise. The numbers give little insight into the relative contribution of the psychosocial factors in the etiology of CHD. Not only is the set of factors included in the analysis rather limited, but psychosocial factors may also affect health through their association with one or more classic risk factors. For example, in Kaunas the mean blood pressure was significantly elevated among those who were born in a small town. Their involuntary migration may have resulted in elevated blood pressure. Adjusting the effect of psychosocial factors for the classic risk factors may be misleading, because in doing so one may adjust a cause for its (intermediate) effect. The association of several psychosocial factors with smoking also points to the environmental determinants of individual lifestyles.

What this suggests for preventive purposes is that psychosocial characteristics can be important in evaluating an individual's risk profile. Knowledge about the social context in which health behaviors and lifestyle originate, develop, and are reinforced can be useful for carrying out public health programs and intervention trials. The relevance of the SES, marital status, and the family culture may be illustrative in this regard. Interventions set up to reduce the level of traditional coronary risk factors could be specifically aimed at people with susceptible positions on any of these environmental characteristics. Intervention programs to change their lifestyle could, then, link up more effectively with the way these groups perceive life. Interventions seem, then, most fruitful, when individuals come to know their own responsibility for their health. Individuals should be made aware of negative health consequences of certain behaviors that are expected and considered appropriate by their peers.

Because not only traditional risk factors were involved in the causal pathway, it is necessary to explore how psychosocial characteristics result in the development of heart disease. The potential importance of more factors was discovered after 1970. Social support, hostility, life events, and lack of control over working conditions or vital exhaustion are such factors, and they may alter the ROC curves. However, in most cases methods of influencing these factors are still being developed. Some of them have been found to be effective in reducing the risk of recurrence in patients afflicted by disease (Friedman et al., 1986). Their efficacy for primary prevention must still be proven, and some of them (family history of CVD, marital status, and SES) can hardly be influenced on an individual level. 


\section{SAMENVATTING}

Onder auspiciën van de Wereldgezondheidsorganisatie werd in de periode 19721974 een cardiovasculair screeningsprogramma gestart in Rotterdam en in Kaunas (een stad in Litouwen; toentertijd één van de republieken van de Sovjet Unie). In de studie, met de naam de Kaunas-Rotterdam Interventie Studie (KRIS), werden vergelijkbare methoden gebruikt om een groot aantal somatische, psychologische en sociologische kenmerken vast te stellen bij 3365 Rotterdamse en 2452 Litouwse mannen tussen de 45 en 60 jaar oud.

Ongeveer tien jaar na de screening bleken 350 Rotterdamse mannen en 303 Litouwse mannen gestorven te zijn. Hiervan waren er respectievelijk 125 en 68 gestorven ten gevolge van een ischemische hartziekte. Daarnaast bleken er 126 en 84 niet-fatale infarcten te zijn opgetreden.

Een zevental psychosociale factoren zijn gedetailleerd onderzocht wat betreft hun potentiële invloed op sterfte en het optreden van een infarct gedurende de tienjarige follow-up:

1. sociaal-economische status

2. sociaal netwerk

3. geografische mobiliteit

4. type A gedrag

5. stress en uitputting

6. subjectief ervaren gezondheid

7. familiale belasting

De analyses werden uitgevoerd met het Cox proportional hazards model ('event history model') en het logistische regressie model. De standaardprocedure was om eerst voor leeftijd te controleren en vervolgens ook de klassieke coronaire risicofactoren (roken, hoge bloeddruk etc.) in het model te introduceren.

\section{Sociaal-economische status}

De opleiding van de mannen bleek relatief zwak samen te hangen met de door ons gebruikte eindpunten. Mannen met een laag beroep hadden echter wel een verhoogd risico op vroegtijdige sterfte ten opzichte van mannen met hogere beroepen; dit verband met beroep was zwakker voor het risico op een niet-fataal infarct. Dit laatste was zeker het geval in Kaunas, waar er geen relatie bestond. Dit kan samen hangen met het feit dat ook in de KRIS de relatie tussen sociaal-economische status en ischemische hartziekten tussen twee generaties van richting veranderd is. Dat wil zeggen, bij de vaders van de participanten kwam het infarct vooral voor bij mannen met een hoge sociaal-economische status, terwijl het bij de participanten de neiging vertoont om juist bij de lagere sociale klassen meer voor te komen. 
Een andere belangrijke bevinding was dat de opleiding van de echtgenote een sterk effect had op het risico op sterfte c.q. een infarct bij gehuwde mannen. Mannen gehuwd met een vrouw met een laag opleidingsniveau bleken een groter risico te hebben dan mannen met hoog opgeleide echtgenoten. Dit effect was het meest geprononceerd voor de totale sterfte en minder voor de cardiovasculaire eindpunten. Deze samenhang bleek onafhankelijk te zijn van het opleidingsniveau van de man zelf.

Alle bovenstaande relaties berustten slechts ten dele op verschillen in de verdeling van klassieke risicofactoren.

\section{Sociaal netwerk}

Burgerlijke staat bleek, onafhankelijk van de klassieke risicofactoren, een sterke relatie te hebben met sterfte en fatale infarcten. Er waren enkele verschillen tussen Nederland en Litouwen in de groepen ongehuwden (nooit gehuwd, weduwnaar en gescheiden) die een speciaal verhoogd risico hadden. In Rotterdam bleken met name de vrijgezellen een verhoogd risico te hebben, terwijl in Kaunas met name de gescheiden mannen en de weduwnaars een verhoogde kans op sterfte hadden.

Alhoewel de gebruikte meting van sociale ondersteuning van vrienden geen hoofdeffect liet zien, werden in beide steden indicaties gevonden voor een beschermend effect van vrienden voor degenen die weduwnaar of gescheiden waren.

\section{Geografische mobiliteit}

Geografische mobiliteit was in beide populaties gerelateerd aan een verhoogde kans op een al dan niet fataal infarct. Degenen die ooit van minder geürbaniseerde gebieden naar Kaunas of Rotterdam verhuisd waren, hadden een hoger risico op een infarct dan degenen die altijd in een grote stad gewoond hadden. Dit verhoogde risico had de neiging om te dalen, wanneer men reeds langer in Rotterdam of Kaunas woonde.

\section{Type A gedrag, stress en uitputting}

Type A gedrag, vastgesteld met behulp van de Jenkins Activity Survey, bleek geen effect te hebben op de eindpunten. Stress, zoals gemeten door de Reeder stress schaal, had ook geen samenhang met de eindpunten. Omdat de resultaten van de Reeder stress schaal in Kaunas geïnvalideerd waren door een response set, moesten de analyses beperkt worden tot het Rotterdamse cohort. Één item van deze schaal dat specifiek vroeg naar gevoelens van uitputting had een sterk voorspellend vermogen ten aanzien van het optreden van een fataal infarct binnen 1 tot 4 jaar. Ook dit verband kon niet verklaard worden door de klassieke risicofactoren. 


\section{Subjectief ervaren gezondheid}

Zowel Litouwse als Nederlandse mannen met een als slecht ervaren gezondheid hadden een verhoogde kans op (coronaire) sterfte tijdens de follow-up periode. Verschillende analyses toonden aan dat deze relatie niet gebaseerd was op een reeds aanwezige (subklinische) ziekte. Deze samenhang kon ook niet verklaard worden door familiale belasting of andere risicofactoren. Ook berustte de samenhang niet op het zich zorgen maken over de gezondheid, ongehuwd zijn of een lage opleiding. Nadere analyses maakten duidelijk dat een verklaring mogelijk kan zijn met behulp van de 'sense of mastery' theorie.

\section{Familiale belasting}

Het gegeven dat ouders een cardiovasculaire aandoening hadden gehad, bleek in beide populaties samen te hangen met het optreden van een infarct bij de KRIS participanten. In Rotterdam had ook het al of niet voorkomen van cardiovasculaire aandoeningen bij broers of zussen een sterk en onafhankelijk, voorspellend vermogen. Deze familiale clustering van cardiovasculaire aandoeningen en met name de rol van (gedeelde) omgevingsfactoren daarin werd ook geilllustreerd door een sterke samenhang tussen de kansen op een cardiovasculaire aandoening bij beide ouders. Verhoogde bloeddruk en cholesterol gehalte en vergelijkbare opleidings- en beroepsniveaus binnen de familie konden deze samenhang niet verklaren. Vroegtijdige sterfte van de ouders bleek geen relatie te hebben met de eindpunten van de KRIS participanten.

Aparte analyses werden gedaan om de Litouwse en Nederlandse mannen met elkaar te vergelijken. Voor de kenmerken die op eenzelfde manier gemeten zijn in beide steden kon worden vastgesteld dat Rotterdammers ouder waren, eenzelfde systolische bloeddruk hadden, een lagere diastolische bloeddruk hadden, meer rookten, minder alcohol dronken, een hoger cholesterol en glucose tolerantie gehalte hadden, slanker waren en minder lichaamsbeweging hadden dan mannen uit Kaunas. Daarnaast waren Rotterdammers vaker nooit gehuwd of gescheiden en hadden ze vaker ouders, broers of zussen die cardiovasculaire aandoeningen hadden. Tenslotte bleken ze veel vaker hun gezondheid als goed te ervaren dan Litouwers. Deze verschillen tussen de populaties werden gerelateerd aan verschillen tussen de populaties in de kans op sterfte en een niet-fataal infarct. Deze analyses toonden aan dat de Litouwers, rekening houdend met alle risicofactoren, een gunstiger cardiovasculair risicoprofiel hadden dan de Rotterdammers. Dit manifesteerde zich in een verlaagde kans op ischemische hartziekten in Litouwen. Het $30 \%$ hoger risico op sterfte gedurende de follow-up in Kaunas kon echter niet verklaard worden door hun risicoprofiel, alhoewel de in het algemeen als slechter ervaren gezondheid in Kaunas een substantiële rol speelde in de hogere mortaliteit in die stad. 


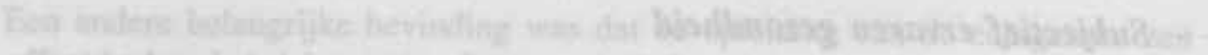

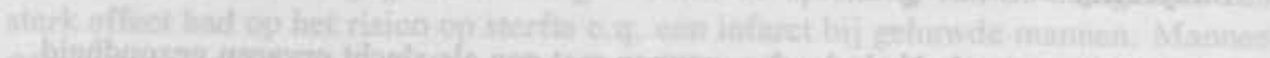

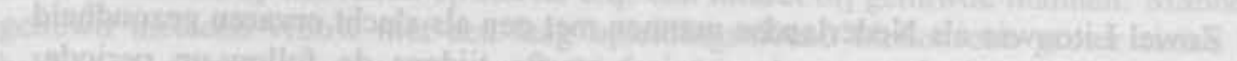

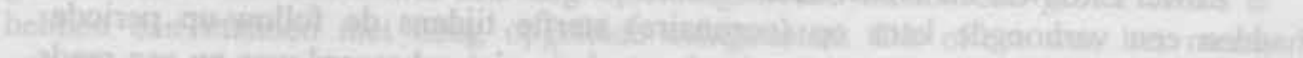

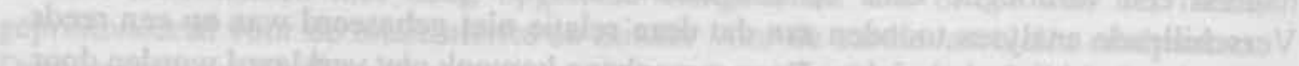

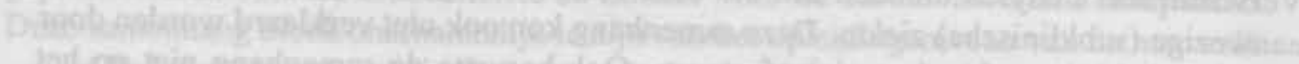

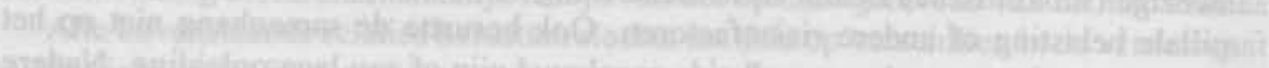

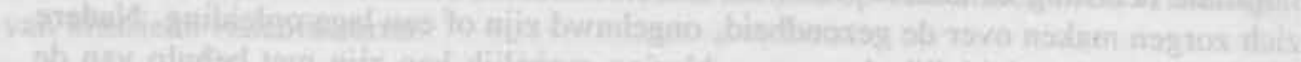

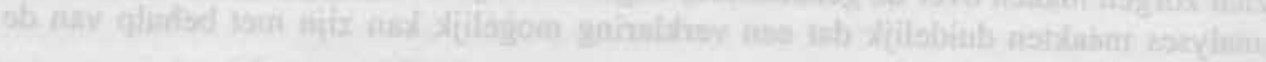

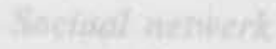

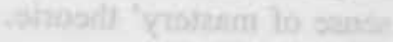

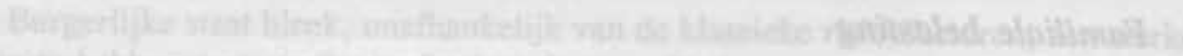

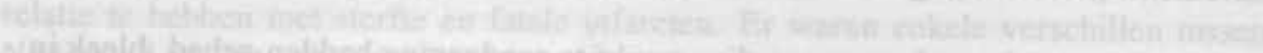

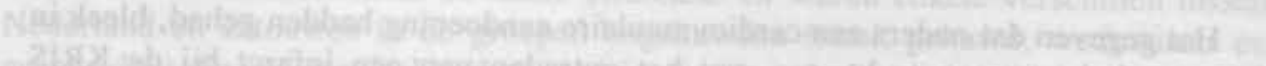

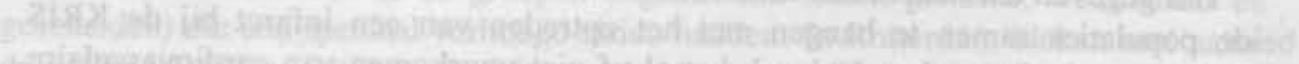

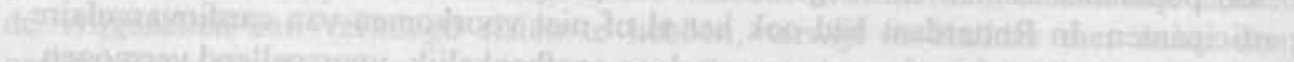

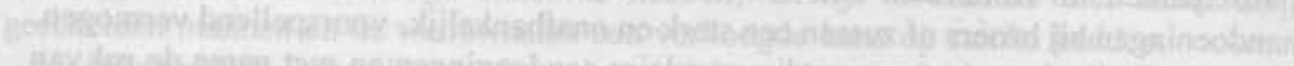

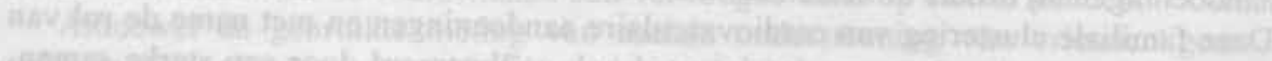

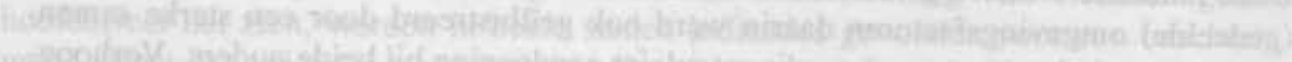

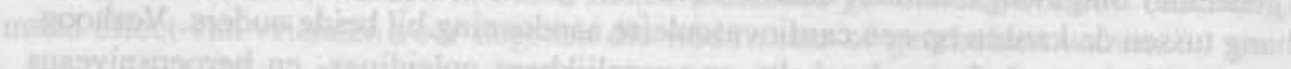

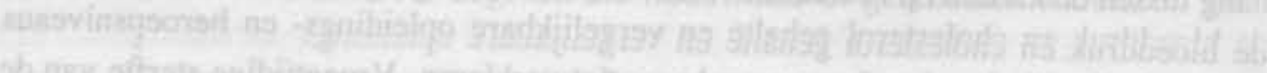

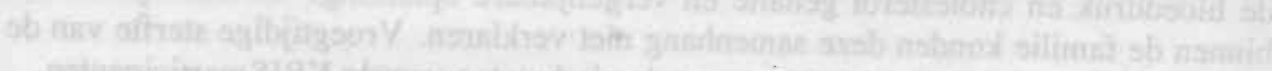

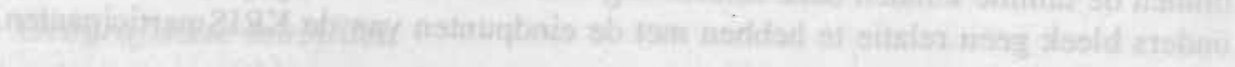

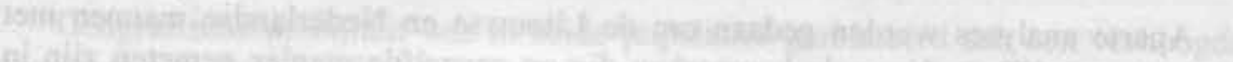

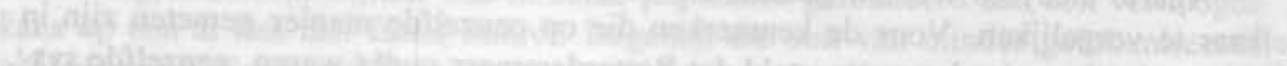

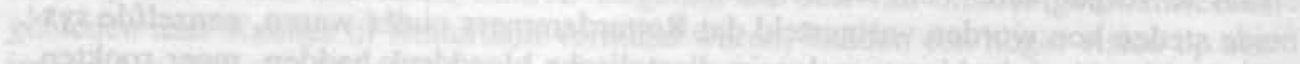

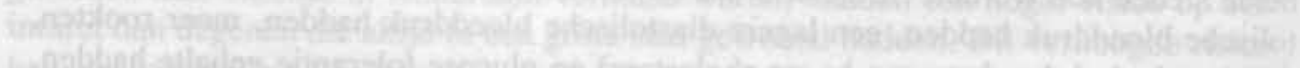

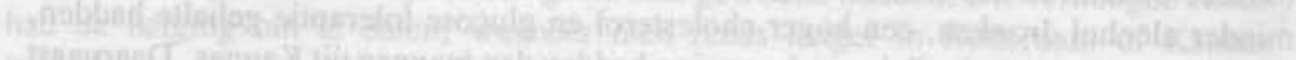

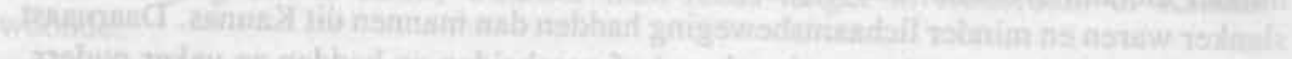

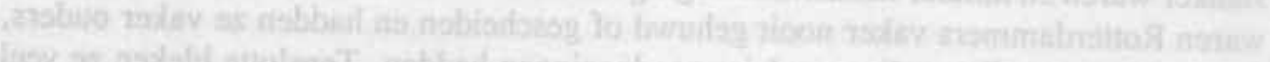

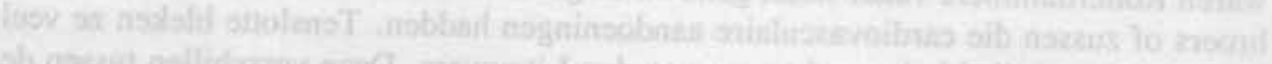

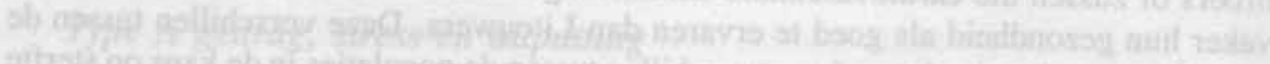

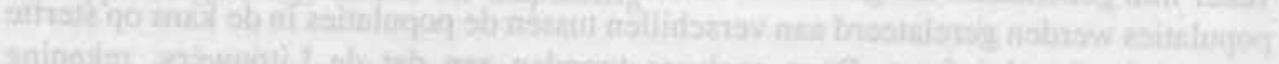

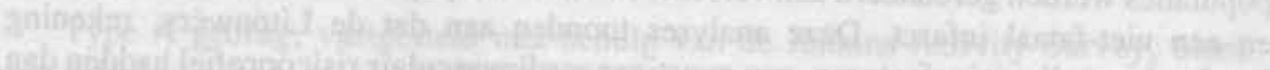

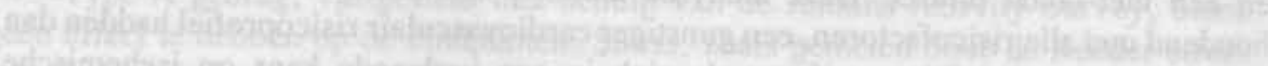

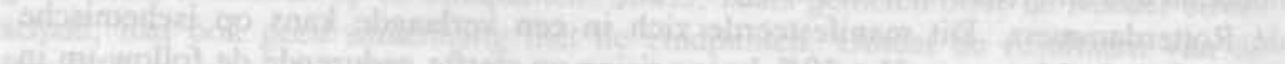

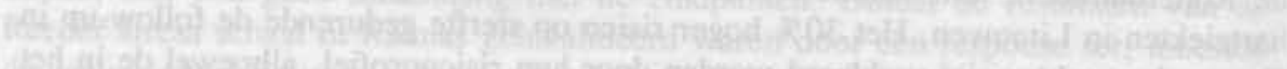

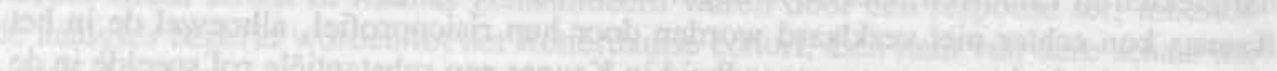

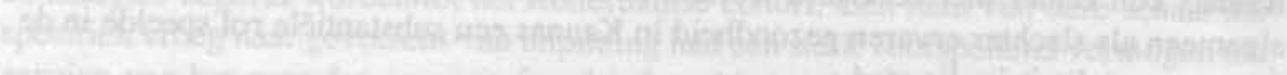

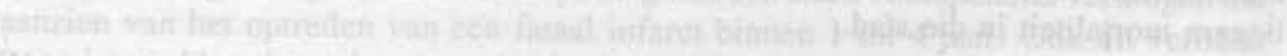

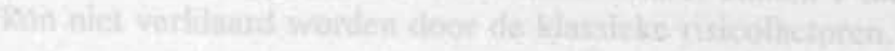




\section{REFERENCES}

Altman, D.G. (1991) Practical statistics for medical research. London: Chapman and Hall. Antonovsky, A. (1968) Social class and the major cardiovascular diseases. Journal of Chronic Diseases, 21, 65.

Appels, A. \& Mulder, P. (1985) Type A behavior and myocardial infarction. A 9.5 year follow-up of a small cohort. International Journal of Cardiology, 8, 465-470.

Appels, A., Mulder, P., Hof, M. van 't, Jenkins, C.D., Houtem, J. van \& Tan, F. (1987) A prospective study of the Jenkins Activity Survey as a risk indicator for coronary heart disease in The Netherlands. Journal of Chronic Diseases, 40, 959-965.

Appels, A. \& Mulder, P. (1988) Excess fatigue as a precursor of myocardial infarction. European Heart Journal, 9, 758-764.

Appels, A. \& Otten, F. (1992) Exhaustion as precursor of cardiac death. Britsh Journal of Clinical Psychology, 31, 351-356.

Appels, A. \& Schouten, E. (1991) Waking up exhausted as risk indicator of myocardial infarction. American Journal of Cardiology, 68, 395-398.

Appels, A., Mulder, P., Otten, F. \& Sturmans F. (1988) De KRIS follow-up study I. Methoden van dataverzameling. Tijdschrift Sociale Gezondheidszorg, 66, 18-21.

Appels, A., Otten, F., Mulder, P., Sturmans, F. \& Schuurman, J. (1988) KRIS follow-up VI. Stress en cardiale mortaliteit. Tijdschrift voor Sociale Gezondheidszorg, 67, 308310.

Appels, A., Otten, F., Sturmans, F., Mulder, P. \& Schuurman, J. (1989) KRIS follow-up V. Effecten van de behandeling van bloeddruk, serum cholesterol en glucose tolerantie. Tijdschrift voor Sociale Gezondheidszorg, 67, 96-99.

Appels, A., Otten, F., Mendes de Leon, C., Sturmans, F., Mulder, P. \& Schuurman, J. (1990) De KRIS follow-up studie VII. Sociaal-economische status en gezondheid. Tijdschrift voor Sociale Gezondheidszorg, 68, 298-305.

Bakker, B.M.F. (1992) Sociale afstand, sociale status. (thesis). Nijmegen: Instituut voor Sociale Wetenschappen.

Bausch-Goldbohm, R.A., Dubbeldam-Marree, A.L., Sturmans, F., Mulder, P., Das, P. \& Boerma, G.J.M. (1983) De Kaunas-Rotterdam Interventie Studie (KRIS) X. Resultaten van de behandeling voor bloeddruk, serumcholesterol en glucosetolerantie bij de deelnemers aan het vijfjarig behandelingsprogramma in Rotterdam. Tijdschrift voor Sociale Gezondheidszorg, 61, 403-410.

Beaglehole, R. (1990) International trends in coronary heart disease mortality, morbidity, and risk factors. Epidemiological Review, 12, 1-15.

Berkman, L.F. \& Syme, S.L. (1979) Social networks, host resistance, and mortality: a nineyear follow-up of Alemada County residents. American Journal of Epidemiology, 109, 186-204.

Berting, J. (1965) status incongruentie and sociale mobiliteit. Sexuologische Gids, 2-8.

Blazer, D. (1982) Social support and mortality in an elderly community population. American Journal of Epidemiology, 115, 684-694.

Blumenthal, J.A., Bradley, W., Dimsdale, J.E., Kasl, S.V., Powel, L.H. \& Taylor, C.B. (1989) Task Force III Assessment of psychosocial status in patients with ischemic heart disease. Journal of the American College of Cardiology, 14, 1016-1042. 
Boerma, G.J.M., Toleikes, A., Mather, A., Bartels, C.T., Margeviciene, L., Glasunov, I.S. \& Leijnse, B. (1978) Laboratory standardization in an international study: the Kaunas Rotterdam Intervention Study (KRIS). Clinical Chemistry, 24, 1126-1130.

Bolton Smith, C., Smith, W.C., Woodward, M. \& Tunstall Pedoe, H. (1991) Nutrient intakes of different social-class groups: results from the Scottish Heart Health Study (SHHS). British Journal of Nutrition, 65, 321-335.

Bonney, N. (1988) Gender, household and social class. British Journal of Sociology, 39, 2846.

Bosma, H., Appels, A., Sturmans, F., Mulder, P. \& Schuurman, J. (1992) KRIS follow-up $\mathrm{XI}$ : De invloed van niet-somatische factoren op de levensverwachting bij personen met tekenen van een hartlijden. Tijdschrift voor Sociale Gezondheidszorg, 70, 293298.

Bowling, A. (1987) Mortality after bereavement: a review of the literature on survival periods and factors affecting survival. Social Science and Medicine, 24, 11-24.

Brand, F.N., Kiely, D.K., Kannel, W.B. \& Myers, R.H. (1992) Family patterns of coronary heart disease mortality: the Framingham Longevity Study. Journal of Clinical Epidemiology, 45, 169-174.

Bray, G.A. (1987) Overweight is risking fate. Definition, prevalence, and risks. Annals of New York Academy of Sciences, 499, 4-13.

Broadhead, W.E., Kaplan, B.H., James, S.A., Wagner, E.H., Schoenbach, V.J., Grimson, R., Heyden, S., Tibblin, G. \& Gehlbach, S.H. (1983) The epidemiologic evidence for a relationship between social support and health. American Journal of Epidemiology, 117, 521-537.

Brown, J. (1986) Evaluation of self and others: self enhancement biases in social judgments. Social Cognition, 4, 353-376.

Burke, G.L., Savage, P.J., Sprafka, J.M., Selby, J.V., Jacobs, D.R., Perkins, L.L., Roseman, J.M., Hughes, G.H. \& Fabsitz, R.R. (1991) Relation of risk factor levels in young adulthood to parental history of disease. The CARDIA study. Circulation, 84, 1445-1446.

Buunk, B.P. \& Yperen, N. van (1991) Referential comparisons, relational comparisons and exchange orientation; their relation to marital satisfaction. Personality and Social Psychology Bulletin, 17, 710-718.

Carmelli, D., Swan, G.E. \& Rosenman, R.H. (1985) The relationship between wives' social and psychologic status and their husbands' coronary heart disease. American Journal of Epidemiology, 122, 90-100.

Carmelli, D., Swan, G.E. Hunt, S.C. \& Williams, R.R. (1989) Cross-spouse correlates of blood pressure in hypertension-prone families in Utah. Journal of Psychsomatic Research, 33, 75-84.

Carney, R., Rich, M., Freedland, K., Saini, J., te Velde, A., Simeone, C. \& Clark, K. (1988) Major depressive disorder predicts cardiac events in patients with coronary heart disease. Psychosomatic Medicine, 50, 627-633.

Central Bureau of Statistics (1960) Typologie van de Nederlandse gemeenten naar urbanisariegraad.

Colditz, G.A., Rimm, E.B., Giovannucci, E., Stampfer, M.J., Rosner, B. \& Willett, W.C. (1991) A prospective study of parental history of myocardial infarction and coronary artery disease in men. American Journal of Cardiology, 67, 933-938. 
Connor, W.D. (1979) Socialism, politics and equality: hierarchy and change in Eastern Europe and the USSR. New York: Columbia University Press.

Cooper, G.R. \& MacDaniel, V. (1970) Standard methods of clinical chemistry VI. New York: Academic Press.

Cooper, R.S. (1981) Rising death rates in the Soviet Union. The impact of coronary heart disease. New England Journal of Medicine, 304, 1259-1265.

Cooper, R.S. (1983) Epidemiologic features of recent trends in coronary heart disease in the Soviet Union. Journal of the American College of Cardiology, 2, 557-564.

Cooper, R.S. (1987) Has the period of rising mortality in the Soviet Union come to an end? International Journal of Health Services, 17, 515-519.

Cooper, R.S. \& Schatzkin, A. (1982) Recent trends in coronary risk factors in the USSR. American Journal of Public Health, 72, 431-440.

Cox, D.R. (1972) Regression models and life tables. Journal of the Royal Statistical Society, 34, Series B, 187-220.

Cox, D.R. \& Oakes, D. (1984) Analysis of survival data. London: Chapman and Hall.

Cutler, S.J. \& Ederer, F. (1958) Maximum utilisation of the life table method in analysing survival. Journal of Chronic Diseases, 8, 699-712.

Dean, A. \& Ensel, W.M. (1982) Modelling social support, life events, competence, and depression in the context of age and sex. Journal of Community Psychology, 10, 392 408.

Deev, A.D. \& Oganov, R.G. (1989) Trends and determinants of cardiovascular mortality in the Soviet Union. International Journal of Epidemiology, 18, S137-S144.

Dirven, H.J., Lammers, J. \& Ultee, W.C. (1991) Working, but still economically dependent?. Luxembourg Income Study: Working Paper Series \#54.

Djordjevic, M.V., Sigountos, C.W., Hoffman, D., Brunnemann, K.D., Kagan, M.R., Bush, L.P., Safaev, R.D., Belitsky, G.A. \& Zaridze, D. (1991) Assessment of major carcinogens and alkaloids in the tobacco and mainstream smoke of USSR cigarettes. International Journal of Cancer, 47, 348-351.

Domarkiene, S, Tamosiunas, A., Gostautas, A \& Grineviciene, L. (1987) Organizational issues of population studies and community programs.

In J. Bluzas (Ed.) Ischemic heart disease. Diagnosis, clinical manifestations and prevention. Vilnius, Mokslas Publishers.

Dressler, W.W. (1985) Psychosomatic symptoms, stress, and modernization: a model. Culture, Medicine and Psychiatry, 9, 257-286.

Eaker, E.D., Haynes, S.G. \& Feinleib, M. (1983) Spouse behavior and coronary heart disease in men: prospective results from the Framingham Heart Study; II Modification of risk in type A husbands according to the social and psychological status of their wives. American Journal of Epidemiology, 118, 23-41.

Eaker, E.D., Pinsky, J. \& Castelli, W.P. (1992) Myocardial infarction and coronary death among women: psychosocial predictors from a 20-year follow-up of women in the Framingham Study. American Journal of Epidemiology, 135, 854-864.

Elford, J., Whincup, P. \& Shaper, A.G. (1991) Early life experience and adult cardiovascular disease: longitudinal and case-control studies. International Journal of Epidemiology, 20, 833-844.

ERICA Research Group (1991) Prediction of coronary heart disease in Europe. The 2nd report of the WHO-ERICA Project. European Heart Journal, 12, 291-297. 
Eyres, S.J. (1972) Spouse social incongruity and health. (thesis). Chapel Hill: University of North Carolina, Department of Epidemiology.

Falger, P.R.J. (1989) Life-span development and myocardial infarction: an epidemiological study. (thesis). Maastricht: University of Limburg.

Farchi, G., Capocaccia, R., Menotti, A. \& Keys, A. (1981) Risk factors changes and coronary heart disease in an observational study. International Journal of Epdimiology, $10,31-40$.

Feuerstein, M., Labbé, E.E. \& Kuczmierczyk (Ed.) (1986) Health psychology. A psychobiological perspecrive. New York: Plenum Press.

Floud, R. (1989) Measuring European inequality: the use of height data.

In J. Fox (Ed.) Health inequalities in European countries. Alderschot: Gower Publishing Company Limited.

Forshdahl, A. (1977) Are poor living conditions in childhood and adolescence an important risk factor for arteriosclerotic heart disease? British Journal of Preventive \& Social Medicine, 31, 91-95.

Fox, J. (Ed.) (1989) Health inequalities in European countries. Alderschot: Gower Publishing Company Limited.

Fraser, G.E. (1978) Sudden death in Auckland. Australian/New Zealand Journal of Medicine, 8, 490-499.

Friedman, M. \& Rosenman, R. (1959) Association of specific overt behavior pattern with blood and cardiovascular findings: blood cholesterol level, blood clotting time, incidence of arcis senilis and clinical coronary artery disease. Journal of the American Medical Association, 169, 1286-1296.

Friedman, M., Thoresen, C.E., Gill, J.E., Ulmer, D., Powell, L.H., Price, V.A., Brown, B., Thompson, L., Rabin, D.D., Breall, W.S., Bourg, E., Levy, R. \& Dixon, T. (1986) Alteration of type A behavior and its effect on cardiac recurrences in post myocardial infarction patients: summary results of the recurrent coronary prevention project. American Heart Journal, 112, 653-665.

Garbe, S. \& Garbe, E. (1990) La sante en URSS a l"heure de la Perestroika. Cah. Socio. Demo. Med., 30, 5-45.

Glasser, M. (1981) Is longevity inherited? Journal of Chronic Diseases, 34, 439-444.

Glasunov, I.S., Dowd, J.E., Baubiniene, A., Grabauskas, V., Sturmans, F. \& Schuurman, J.H. (1981) The Kaunas Rotterdam Intervention Study. Amsterdam: Elsevier/NorthHolland Biomedical Press.

Goldberg, R.J. \& Novack, D.H. (1992) The psychosocial review of systems. Social Science and Medicine, 35, 261-269.

Gove, W.R. (1973) Sex, marital status and mortality. American Journal of Sociology, 79, 45-67.

Grand, A., Grosclaude, P., Bocquet, H., Pous, J. \& Albarede, J.L. (1990) Disability, psychosocial factors and mortality among the elderly in a rural French population. Journal of Clinical Epidemiology, 43, 773-782.

Grech, E.D., Ramsdale, D.R., Bray, C.L. \& Faragher, E.B. (1992) Family history as an independent risk factor of coronary artery disease. European Heart Journal, 13, 1311-1315.

Hamby, R.I.H. (1981) Hereditary aspects of coronary artery disease. American Heart Journal, 101, 639-649. 
Hassan, J. (1989) Way-of-life, stress and differences in morbidity between occupational classes.

In: J. Fox (Ed.) Health inequalities in European countries. Alderschot: Gower Publishing Company Limited.

Haynes, S. \& Matthews, K. (1988) Review and methodological critique of recent studies on type A behavior and cardiovascular disease. Annals of Behavioral Medicine, 10, 4759.

Haynes, S.G., Feinleib, M. \& Kannel W.B. (1980) The relationship of psychosocial factors to coronary heart disease in the Framingham Study III. 8-year incidence of coronary heart disease. American Journal of Epidemiology, 111, 37-58.

Haynes, S.G., Eaker, E.D. \& Feinleib, M. (1983) Spouse behaviour and coronary heart disease in men: prospective results from the Framingham Heart Study; I Concordance of risk factors and the relationship of psychosocial status to coronary incidence. American Journal of Epidemiology, 118, 1-21.

He, J., Klag, M.J., Whelton, P.K., Chen, J.Y., Mo, J.P., Qian, M.C., Mo, P.S. \& He, G.Q. (1991) Migration, blood pressure pattern, and hypertension: the Yi Migrant Study. American Journal of Epidemiology, 134, 1085-1101.

Hedblad, B., Ostergren, P.O., Hanson, B.S. \& Janzon, L. (1992) Influence of social support on cardiac event rate in men with ischaemic type ST segment depression during ambulatory 24-h long-term ECG recording. The prospective population study in 'Men born in 1914', Malmo, Sweden. European Heart Journal, 13, 433-439.

Heinemann, L., Bulyzhenkov, V. \& Berg, K. (1990) Environmental and genetic risk factors for coronary heart disease. World Review of Nutrition and Dietetics, 63, 84-89.

Henderson, S. (1981) Social relationships, adversity and neurosis, an analysis of prospective observations. British Journal of Psychology, 138, 391-398.

Hirdes, J.P. \& Forbes, W.F. (1992) The importance of social relationships, socioeconomic status and health practices with respect to mortality among healthy Ontario males. Journal of Clinical Epidemiology, 45, 175-182.

Hofmans, M.D.A.F., Kromhout, D. \& de Lezenne Coulander, C. (1989) Body mass index at the age of 18 and its effects on 32-year-mortality from coronary heart disease and cancer. Journal of Clinical Epidemiology, 42, 513-520.

Hoogendoorn, D. (1990) Enkele opmerkingen over de stand van zaken betreffende de epidemie van het acute hartinfarct. Nederlands Tijdschrijf voor de Geneeskunde, 134, $592-595$.

Hosmer, D.W. \& Lemeshow, S. (1989) Applied Logistic Regression. New York: John Wiley $\&$ Sons.

House, J.S., Robbins, C. \& Metzner, H.L. (1982) The association of social relationships and activities with mortality: prospective evidence from the Tecumseh Community Health Study. American Journal of Epidemiology, 116, 123-140.

House, J.S., Landis, K.L. \& Umberson, D. (1988) Social relationships and health. Science, $241,540-544$.

Huang, T., Chen, C.P., Wefler, V. \& Raftery, A. (1961) A stable reagent for the Liebermann-Burchard reaction. Application to rapid serum cholesterol determination. Analytical Chemistry, 33, $1405-1407$.

Hultman, E. (1959) Rapid specific method for determination of aldosaccharides in body fluids. Nature, 183, 108-109. 
Idler, E.L. \& Angel, R.J. (1990) Self-rated health and mortality in the NHANES-I epdimiologic follow-up study. American Journal of Public Health, 80, 446-452.

Idler, E.L. \& Kasl, S. (1991) Health perceptions and survival: do global evaluations of health status really predict mortality? Journal of Gerontology, 46, S55-S65.

Idler, E.L., Kasl, S.V. \& Lemke, J.H. (1990) Self-evaluated health and mortality among the elderly in New Haven, Connecticut, and Iowa and Washington Counties, Iowa, 19821986. American Journal of Epidemiology, 131, 91-103.

Jagger, C. \& Sutton, C.J. (1991) Death after marital bereavement-is the risk increased? Staristics in Medicine, 10, 395-404.

Jenkins, C.D. (1966) Group differences in perception: a study of community beliefs and feelings about tuberculosis. American Journal of Sociology, 71, 417-429.

Jenkins, C.D., Zyzanski, S. \& Rosenman, R. (1979) Jenkins Activity Survey Manual. New York: the Psychological Corporation.

Jones, R.D. (1987) Heart disease mortality following widowhood: some results from the OPCS Longitudinal Study. Journal of Psychosomatic Research, 31, 325-333.

Kalbfleish, J.D. \& Prentice, R.L. (1980) The statistical analysis of failure time data. New York: Wiley.

Kannel, W.B., Neaton, J.D., Wentworth, D., Thomas, H.E., Stamler, J., Hulley, S.B. \& Kjelsberg, M.O. (1986) Overall and coronary heart disease mortality rates in relation to major risk factors in 325,348 men screened for the MRFIT. American Heart Journal, 112, 825-836.

Kaplan, E.L. \& Meier, P. (1958) Nonparametric estimation from incomplete observations. Journal of the American Statistical Association, 53, 457-481.

Kaplan, G.A. \& Camacho, T. (1983) Perceived health and mortality: nine-year follow-up of the Human Population Laboratory cohort. American Journal of Epidemiology, 117, 292-304.

Kaplan, G., Barell, V. \& Lusky, A. (1988) Subjective state of health and survival in elderly adults. Journal of Gerontology, 43, S114-S120.

Kaplan, G.A., Salonen, J.T., Cohen, R.D., Brand, R.J., Syme, S.L. \& Pushka, P. (1988) Social connections and mortality from all causes and from cardiovascular disease: prospective evidence from Eastern Finland. American Journal of Epidemiology, 128, 370-380.

Kate ten, L.P., Boman, H., Daiger, S.P. \& Motulsky, A.G. (1982) Familial aggregation of coronary heart disease and its relation to known genetic risk factors. American Journal of Cardiology, 50, 945-953.

Kato, I., Tominaga, S. \& Suzuki, T. (1990) Correspondence in cancer history between husbands and wives. Japanese Journal of Cancer Research, 81, 584-589.

Kawachi, I., Marshall, S. \& Pearce, N. (1991) Social class inequalities in the decline of coronary heart disease among New Zealand men, 1975-1977 to 1985-1987. International Journal of Epidemiology, 20, 393-398.

Keller, W.J. \& Verbeek, A.J. (1984) ANOTA: analysis of tables. Kwantitatieve Methoden, 15.

Kiecolt, G.J.K., Kennedy, S. \& Malkoff, S., Fischer, L., Speicher, C.E. \& Glaser, R. (1988) Marital discord and immunity in males. Psychosomatic Medicine, 50, 213-229.

Kleinbaum, D.G. (1989) Survival Analysis Module Series. Part I. Introduction to survival analysis. Chapel Hill: University of North Carolina. 
Kleinbaum, D.G., Kupper, L.L. \& Morgenstern, H. (1982) Epidemiologic research. Principles and quantitative methods. New York: Van Nostrand Reinhold Company. Klinger, A. (1986) Socio-economic differentials of mortality. International Seminar on the Socio-Economic Aspects of Differential Mortality, 17-63.

Kop, W.J. (1994) The predictive value of vital exhaustion in the clinical course after coronary angioplasty. (thesis). Maastricht: University of Limburg.

Kraus, A.S. \& Lilienfeld, A.M. (1959) Some epidemiological aspects of the high mortality rate in the young widowed group. Journal of Chronic Diseases, 10, 207-217.

Kuller, L.H. (1978) Prodromota of sudden death and myocardial infarction. Advances in Cardiology, 25, 61-72.

Kunst, A.E. \& Mackenbach, J.P. (1990) Ischemische hartziekte: van managers- tot volksziekte. Een overzicht van de bevindingen uit de internationale literatuur. In: J.P. Mackenbach (Ed.) Sociaal-economische gezondheidsverschillen onderzocht: deel II. Den Haag: Distributiecentrum DOP.

Kunst, A.E., Looman C.W.N. \& Mackenbach J.P. (1990) Socio-economic mortality differences in The Netherlands in 1950-1984: a regional study of cause-specific mortality. Social Science and Medicine, 31, 141-152.

Ladwig, K. (1989) Patient- und Artzreaktionen auf spezifische und unspezifische Warnsignale in der Vorphase eines akuten Myokardinfarktes. Verhaltensmodifikation und Verhaltensmedizin. 10, 181-195.

Lauer, R.M., Burns, T.L., Clarke, W.R. \& Mahoney, L.T. (1991) Childhood predictors of future blood pressure. Hypertension, 18 (3 suppl), 174-81.

Leon, C. \& Wilkinson, R.G. (1989) Inequalities in prognosis: socio-economic differences in cancer and heart disease survival.

In: J. Fox (Ed.) Health inequalities in European countries. Alderschot: Gower Publishing Company Limited.

Liebrand, W.B.G., Messick, D.M. \& Wolters, F.J.M. (1986) Why we are fairer than other: A cross-cultural replication and extension. Journal of Experimental Social Psychology, 22, 590-604.

Lindberg, G., Rastam, L., Gullberg, B. \& Eklund, G.A. (1992) Low serum cholesterol concentration and short term mortality from injuries in men and women. British Medical Journal, 305, 277-279.

Lindholm, L., Lanke, J. \& Bengtsson, B. (1986) U-shaped association of mortality and blood pressure in a thirteen-year prospective study. Family Practice, 3, 3-8.

Lolas, F. \& Mayer, H. (Ed.) (1987) Perspectives on stress and stress-related topics. Berlin: Springer.

Lopez, A.D. (1990) Causes of death: an assessment of global patterns of mortality around 1985. World Health Statistics Quarterly, 43, 91-104.

Lundberg, O. (1991) Causal explanations for class inequality in health: an empirical analysis. Social Science and Medicine, 32, 385-393.

Lundberg, U., Rasch, B. \& Westermark, D. (1990) Familial similarity in Type A behaviour and physiological measurements as related to sex. Scandinavian Journal of Psychology, 31, 34-41.

Lynch, J.J. (1985) Her gebroken har. De medische gevolgen van eenzaamheid. Utrecht.

Mackenbach, J.P. (1992) Socio-economic health differences in The Netherlands: a review of recent empirical findings. Social Science and Medicine, 34, 213-226. 
Mackenbach, J.P., Looman, C.W.N. \& Kunst A.E. (1989) Geographic variation in the onset of decline of male ischemic heart disease mortality in The Netherlands. American Journal of Public. Health, 79, 1621-1627.

Marmot, M.G. \& McDowall, M.E. (1986) Mortality decline and widening social inequalities. Lancet II, 274-276.

Marmot, M.G., Adelstein, A.M. \& Robinson, N. (1978) Changing social-class distribution of heart disease. British Medical Journal, 2, 1109-1112.

Marmot, M.G., Shipley, M.J. \& Rose, G. (1984) Inequalities in death, specific explanations of a general pattern? Lancet $i i, 1003-6$.

Matthews, K. \& Haynes, S. (1986) Type A behavior pattern and coronary disease risk: update and critical evaluation. American Journal of Epidemiology, 123, 923-960.

Matthews, M. (1972) Class and society in Soviet Russia. New York: Walker and Company.

Medalie, J., Kahn, H., Neufeld, H., Riss, E. \& Goldbourt, U. (1973) Five year myocardial infarction incidence II: association of single variables to age and birth place. Journal of Chronic Diseases, 26, 329-349.

Mendes de Leon, C.F., Appels, A.W.P.M., Otten, F.W.J. \& Schouten, E.G.W. (1992) Risk of mortality and coronary heart disease by marital status in middle-aged men in The Netherlands. International Journal of Epidemiology, 21, 460-466.

Mezentseva, E. \& Rimachevskaya, R. (1990) The Soviet country profile: health of the U.S.S.R. population in the 70 s and $80 \mathrm{~s}$-an approach to a comprehensive analysis. Social Science and Medicine, 31, 867-877.

Mezentseva, E. \& Rimachevskaya, R. (1992) The health of the populations in the republics of the former Sovjet Union. International Journal of Health Sciences, 3, 127-142.

Miller, R.G. (1981) Survival analysis. New York: Wiley.

Minev, D., Dermendjieva B. \& Mileva, N. (1990) The Bulgarian country profile: the dynamics of some inequalities in health. Social Science and Medicine, 31, 837-846.

Morgenstern, H. (1980) The changing association of social status and coronary heart disease in a rural population. Social Science and Medicine, 14A, 191-201.

Mossey, J.M. \& Shapiro, E. (1982) Self-rated health: a predictor of mortality among the elderly. American Journal of Public Health, 71, 800-808.

Mulder, P.G.H. (1993) The evaluation of chronic disease intervention. (thesis). Rotterdam: Erasmus University.

Nerbrand, C., Olsson, L., Svardsudd, K., Kullman, S. \& Tibblin, G. (1991) Are regional variations in ischaemic heart disease related to differences in coronary risk factors? European Heart Journal, 12, 309-314.

Notkola, V., Punsar, S., Karvonen, M.J. \& Haapakosky, J. (1985) Socio-economic conditions in childhood and mortality and morbidity caused by coronary heart disease in adulthood in rural Finland. Social Science and Medicine, 21, 517-523.

Nyström Peck, A.M. (1992) Childhood environment, intergenerational mobility, and adult health-evidence from Swedish data. Journal of Epidemiology and Community Health, 46, 71-74.

Office of Population Censuses and Surveys (1978) Occupational mortality 1970-72 England and Wales. London: Her Majesty's Stationery Office.

Olsen, O. \& Kristensen, T.S. (1991) Impact of work environment on cardiovascular diseases in Denmark. Journal of Epidemiology and Communiry Health, 45, 4-10.

Orosz, E. (1990) The Hungarian country profile: inequalities in health and health care in Hungary. Social Science and Medicine, 31, 847-857. 
Orth-Gomer, K. \& Johnson, J. (1987) Social network interaction and mortality. A six year follow-up study of a random sample of the Swedish population. Journal of Chronic Diseases, 40, 949-958.

Ortmeyer, C.F. (1974) Variations in mortality, morbidity and health care by marital status. In: C.E. Erhardt \& J.E. Berlin (Ed.) Mortality and morbidity in the United States. Cambridge MA: Harvard University Press.

Östberg, V. \& Vagerö, D. (1991) Socio-economic differences in mortality among children. Do they persist into adulthood? Social Science and Medicine, 32, 403-410.

Otten, F., Reek, J. van, Appels, J., Mulder, P. \& Sturmans, F. (1988) KRIS follow-up studie III. De effecten van roken op totale mortaliteit, hartinfarct en kanker van de luchtwegen. Tijdschrift voor Sociale Gezondheidszorg, 66, 154-156.

Parkes, C.M., Benjamin, B. \& Fitzgerald, R.G. (1969) Broken heart: a statistical study of increased mortality among widowers. British Medical Journal, 1, 740-743.

Paykel, E.S., Emmus, E.M. \& Fletcher, J. (1980) Life events and social support in puerperal depression. British Journal of Psychology, 136, 339-346.

Pearlin, L.I., Lieberman, M.A. \& Menaghan E.G. (1981) The stress process. Journal of Health and Social Behavior, 22, 337-356.

Perkins, K.A. (1989) Interactions among coronary heart disease risk factors. Annals of Behavioral Medicine, 11, 3-11.

Peterson, C. \& Seligman, M.E.P. (1987) Explanatory style and illness. Journal of Personaliry, $55,237-265$.

Power, C. (1991) Social and economic background and class inequalities in health among young adults. Social Science and Medicine, 32, 411-417.

Prenger, V.L., Beaty, T.H. \& Kwiterovich, P.O. (1992) Genetic determination of highdensity lipoprotein-cholesterol and apolipoprotein A-1 plasma levels in a family study of cardiac catheterization patients. American Journal of Human Genetics, 51, 1047 1057.

Price, W.M., Morris, S.W., Kitchen, A.H., Wenham, P.R., Burgon, P.R.S. \& Donald, P.M. (1989) DNA restriction fragment length polymorphisms as markers of familial coronary heart disease. Lancet ii, 1407-1411.

Prokhorskas, R. (1992) The concept of generalized risk and some results of preliminary analysis.

In: W. Morgenstern, E. Chigan, R. Prokhorskas, M. Rusnak \& G. Schettler (Ed.) Models of noncommunicable diseases. Berlin: Springer-Verlag.

Ranchor, A.V., Sanderman, R. \& Heuvel, W.J.A. van den (1990) An integrative appraoch to inequality in health: a longitudinal study encompassing SES, lifestyle, personality and health. Internarional Journal of Health Sciences, 1, 121-135.

Reed, D., McGee, D. \& Yano, K. (1984) Psychosocial processes and general susceptiblity to chronic disease. American Journal of Epidemiology, 119, 356-370.

Reeder, L.G., Chapman, J.M. \& Coulson, A.H. (1968) Socio-environmental stress, tranquilizers and cardiovascular disease. Proceedings of the Excerpta Medica International Congress Series No. 182, Baia Domizia, 226-238.

Reiff, G.G., Montoye, H.J., Remington, R.D., Napier, J.A., Metzner, H.L. \& Epstein, F.H. (1964) Assessment of physical activity by questionnaire and interview.

In: M.J. Karvonen \& A.L. Barry (Ed.) Physical activity and the heart. Proceedings of a Symposium, Helsinki. Springfield, Ill., Charles C. Thomas. 
Rissanen, A.M., Nikkilä, E.A. (1979) Aggregation of coronary risk factors in families of men with fatal and non-fatal coronary heart disease. British Heart Journal, 42, 373380.

Rissanen, A., Heliovaara, Knekt, P., Aromaa, A., Reunanen, A. \& Maatela, J. (1989) Weight and mortality in Finnish men. Journal of Clinical Epidemiology, 42, 781-789.

Rose, G.A. \& Blackburn, H. (1968) Cardiovascular survey methods. Monograph no. 56, Geneva: World Health Organization.

Rosengren, A., Wedel, H. \& Wilhelmsen, H. (1988) Marital status and mortality in middleaged Swedish men. American Journal of Epidemiology, 129, 54-64.

Rosenman, R. \& Friedman, M. (1961) Association of specific behavior pattern in women with blood and cardiovascular findings. Circulation, 24, 1173-1184.

Rosenman, R., Brand, R., Jenkins, C.D., Friedman, M., Straus, R. \& Wurm, M. (1975) Coronary heart disease in the Western Collaborative Group Study: final follow-up experience of 8.5 years. Journal Of the American Medical Association, 233, 872-877.

Ruberman, W., Weinblatt, E., Goldberg, J.D. \& Chaudhary, B.S. (1984) Psychosocial influences on mortality after myocardial infarction. New England Journal of Medicine, 311, 552-559.

Ruzicka, L. \& Lopez, A.D. (1990) The use of cause-of-death statistics for health situation assessment: national and international experiences. World Health Statistics Quarterly, 41, 249-258.

Sackett, D.L., Haynes, R.B., Guyatt, G.H. \& Tugwell, P. (1991) Clinical epidemiology: $a$ basic science for clinical medicine. Boston: Little, Brown and Company.

Salmond, C.E., Prior, I.A.M. \& Wessen, A.F. (1989) Blood pressure patterns and migration: a 14-year cohort study of adult Tokelauans. American Journal of Epidemiology, 130, 37-52.

Schildkraut, J.M., Meyers, R.H., Cupples, L.A., Kiely, D.K. \& Kannel, W.B. (1989) Coronary risk factors with age and sex of parental heart disease in the Framingham Study. American Journal of Cardiology, 64, 555-559.

Shekelle, R.B., Ostfeld, D.A. M. \& Oglesby, P. (1969) Social status and incidence of coronary heart disease. Journal of Chronic Diseases, 22, 381-394.

Sholtz, R.I., Rosenman, R.H. \& Brand, R.J. (1975) The relationship of reported parental history to the incidence of coronary heart disease in the Western Collaborative Group Study. American Journal of Epidemiology, 102, 350-356.

Siegrist, J. (1989) Steps towards explaining social differentials in morbidity: the case of West Germany.

In: J. Fox (Ed.) Health inequalities in European countries. Alderschot: Gower Publishing Company Limited.

Silverstein, M. \& Bengtson, V.L. (1991) Do close parent-child relations reduce the mortality risk of older parents? Journal of Health and Social Behavior, 32, 382-395.

Simon, J., Cajzi, J., Krizanovska, M., Krizek, M. \& Kraus, J. (1986) Occupation and education in relation to risk factors of ischaemic heart disease in the male industrial population. Cor Vasa, 28, 167-176.

Slack, J. \& Evans, K.A. (1966) The increased risk of death from ischaemic heart disease in the first degree relatives of 121 men and 96 women with ischaemic heart disease. Journal of Medical Generics, 3, 239-257. 
Slater, C.H., Lorimor R.J. \& Lairson D.R. (1985) The independent contributions of socioeconomic and health practices to health status. Preventive Medicine, 14, $372-$ 378.

Snowden, C.B., McNamara, P.M., Garrison, R.J., Feinleib, M., Kannel, W.B. \& Epstein, F.H. (1982) Predicting coronary heart disease in siblings; a multivariate assessment. American Journal of Epidemiology, 115, 217-222.

Sorensen, T.J.A., Nielsen, G.G., Andersen, P.K. \& Teasdale, T.W. (1988) Genetic and environmental influences on premature death in adult adoptees. New England Journal of Medicine, 318, 727-732.

Steinbach, U. (1992) Social networks, institutionalization, and mortality among elderly people in the United States. Journal of Gerontology, 47, S183-S190.

Stroebe, W. \& Stroebe, M.S. (1987) Bereavement and health. The psychological and physical consequences of partner loss. Cambridge UK: Cambridge University Press.

Strogatz, D.S., Siscovick, D.S., Weiss, N.S. \& Rennert, G. (1988) Wife's level of education and husband's risk of primary cardiac arrest. American Journal of Public Health, 78, 1491-1493.

Stronks, K., Mheer, H. van de, \& Mackenbach, J.P. (1993) Achtergronden van sociaaleconomische gezondheidsverschillen. Een overzicht van de literatuur en een onderzoeksmodel. Tijdschift voor Sociale Gezondheidszorg, 71, 2-10.

Suarez, L. \& Barret-Conner, E. (1984) Is an educated wife hazardous to your health? American Journal of Epidemiology, 119, 244-249.

Svenson, O. (1981) Are we all less risky and more skillful than our fellow drivers? Acta Psychologica, 47, 143-148.

Syme, S.L., Hyman, M.M. \& Enterline, P.E. (1964) Some social and cultural factors associated with the occurrence of coronary heart disease. Journal of Chronic Diseases, 17, 277-289.

Thomas, P.D., Goodwin, J.M. \& Goodwin, J.S. (1985) Effect of social support on stressrelated changes in cholesterol level, uric acid level and immune function in an elderly sample. American Journal of Psychiatry, 142, 735-737.

Uemura, K. \& Pisa, Z. (1988) Trends in cardiovascular disease mortality in industrialized countries since 1950. World Health Statistics Quarterly, 41, 155-178.

Ultee, W.C., Arts, W. \& Flap, H. (1992) Sociologie: vragen, uitspraken, bevindingen. Groningen: Wolters-Noordhoff.

Umberson, D. (1992) Gender, marital status and the social control of health behavior. Social Science and Medicine, 34, 907-917.

Vagerö, D. (1991) Inequality in health; some theoretical and empirical problems. Social Science and Medicine, 32, 367-371.

Vagerö, D. \& Norell, S. (1989) Mortality and social class in Sweden; exploring a new epidemiological tool. Scandinavian Journal of Social Medicine, 17, 49-58.

Vandenbroucke, J.P., Matroos, A.W., Heide-Wessel, C. van der, \& Heide, R.M. van der (1984) Parental survival, an independent predictor of longevity in middle-aged persons. American Journal of Epidemiology, 119, 742-750.

Vaupel, J.W. (1988) Inherited frailty and longevity. Demography, 25, 227-287.

Vernon, S.W. \& Buffler, P.A. (1988) The status of status inconsistency. Epidemiologic reviews, $10,65-86$.

Virganskaya, I.M. \& Dmitriev, V.I. (1992) Some problems of medicodemographic development in the former Soviet Union. World Health Statistics Quarterly, 45, 4-14. 
Vogt, T.M., Mullooly, J.P., Emst, D., Pope, C.R. \& Hollis, J.F. (1992) Social networks as predictors of ischemic heart disease, cancer, stroke and hypertension: incidence, survival and mortality. Journal of Clinical Epidemiology, 45, 659-666.

Wannamethee, G. \& Shaper, A.G. (1991) Self-assessment of health status and mortality in middle-aged British men. International Journal of Epidemiology, 20, 239-245.

Welin, L., Tibblin, G., Svardsudd, K., Tibblin, B., Ander-Peciva, S., Larsson, B. \& Wilhelmsen, L. (1985) Prospective study of social influences on mortality. The study of men born in 1913 and 1923. Lancet ii, 915-918.

Welin, L., Larsson, B., Svardsudd, K., Tibblin, B. \& Tibblin, G. (1992) Social network and activities in relation to mortality from cardiovascular diseases, cancer, and other causes: a 12 year follow-up of the study of men bom in 1913 and 1923. Journal of Epidemiology and Communiry Health, 46, 127-132.

Wertlieb, D., Budman, S., Demby, A. \& Randall, M. (1982) The stress of marital separation; intervention in a health maintenance organization. Psychosomatic Medicine, 44, 437-448.

West, P. (1991) Rethinking the health selection explanation for health equalities. Social Science and Medicine, 32, 373-384.

Westerlaak, J.M. van, Kropman, J.A. \& Collaris, J.W.M. (1975) Beroepenklapper. Nijmegen: Instituut voor Toegepaste Sociologie.

Wilkinson, R.G. (1990) Income distribution and mortality: a 'natural" experiment. Sociology of Health and Illness, 12, 391-412.

Williams, R.B., Barefoot, J.C., Califf, R.M., Haney, T.L., Saunders, W.B., Pryor, D.B., Hlatky, M.A., Siegler, I.C. \& Mark, D.B. (1992) Prognostic importance of social and economic resources among medically treated patients with angiographically documented coronary artery disease. Journal of the American Medical Association, 267, 520-524.

Williams, R.R., Hunt, S.C., Hasstedt, S.J., Hopkins, P.N., Wu, L.L., Berry, T.D., Stults, B.M., Barlow, G.K. \& Kuida, H. (1990, Genetics of hypertension: what we know and don't know. Clinical and Experimental Hypertension (part A), 12, 865-876.

Williams, R.R., Hopkins, P.N., Hunt, S.C., Wu, L.L., Hasstedt, S.J., Lalouel, J.M., Ash, K.O., Stults, B.M. \& Kuida, H. $\left(1990_{b}\right)$ Population-based frequency of dyslipidemia syndromes in coronary-prone families in Utah. Archives of Internal Medicine, 150, $582-588$.

Williams, R.R., Hunt, S.C., Hasstedt, S.J., Hopkins, P.N., Wu, L.L., Berry, T.D., Stults, B.M., Barlow, G.K., Schumacher, M.C., Lifton, R.P. \& Laouel, J.M. (1991) Are there interactions and relations between genetic and environmental factors predisposing to high blood pressure. Hypertension, 18 ( 3 suppl), 129-37.

Williamson, R. \& Kessling, A.M. (1990) The problem of polygenic disease. Ciba Foundation Symposia, 149, 63-80.

Wnuk-Lipinski, E. (1990) The Polish country profile: economic crisis and inequalities in health. Social Science and Medicine, 31, 859-866.

Wnuk-Lipinksi, E. \& Illsley, R. (1990) International comparative analysis: main findings and conclusions. Social Science and Medicine, 31, 879-889.

World Health Organization (1967) Manual of the international statistical classification of diseases, injuries and causes of death. Eighth Revision. Geneva: World Health Organization. 
World Health Organization (1977, Manual of the international statistical classification of diseases, injuries and causes of death. Ninth Revision. Geneva: World Health Organization.

World Health Organization (1977 $)$ Manual of mortality analysis: a manual on methods of analysis of national mortality statistics for public health purposes. Geneva: World Health Organization.

Zaridze, D.G. \& Basieva, T.H. (1990) Incidence of cancer of the lung, stomach, breast, and cervix in the USSR: pattern and trends. Cancer Causes and Control, 1, 39-49. 


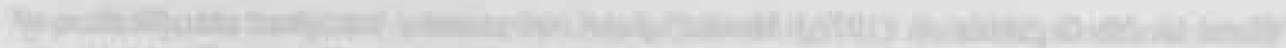
thath

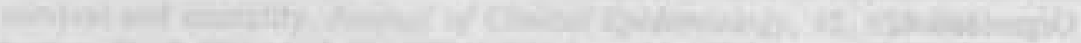

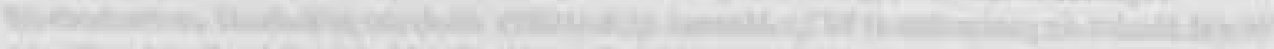

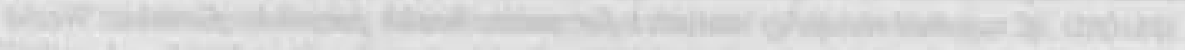
F⿻十

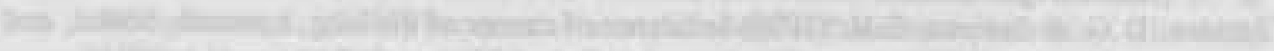

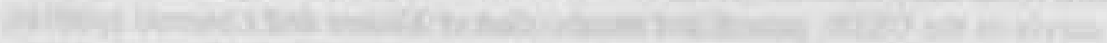

that and

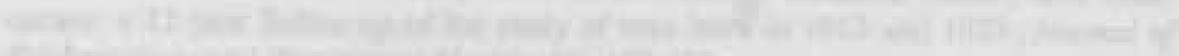

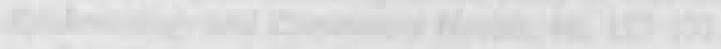

and

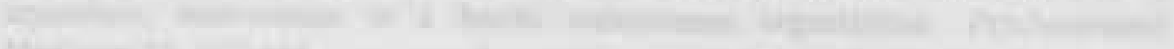

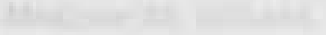

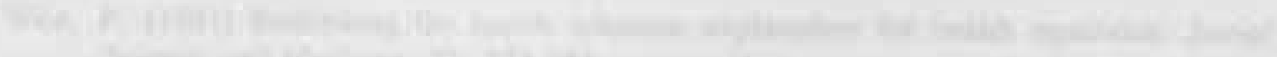

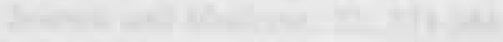

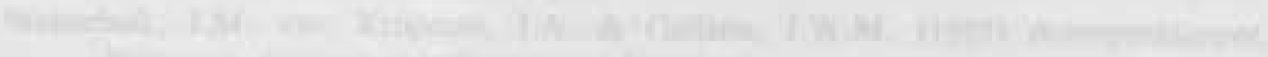

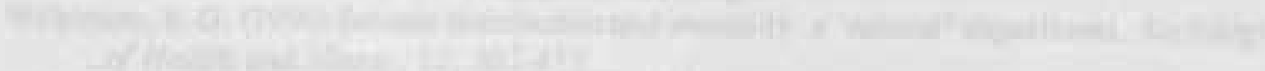

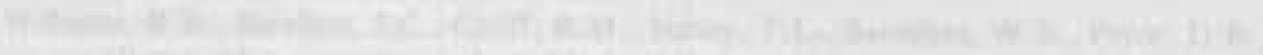

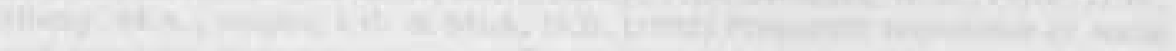

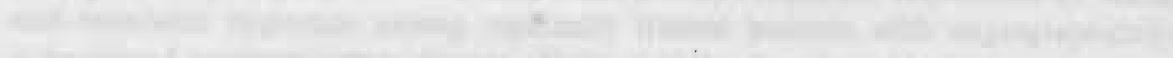
10 fra

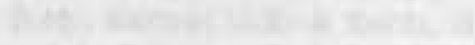

tan

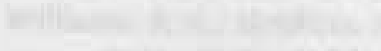

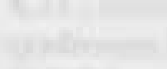

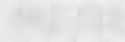

Thith

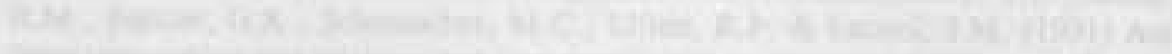

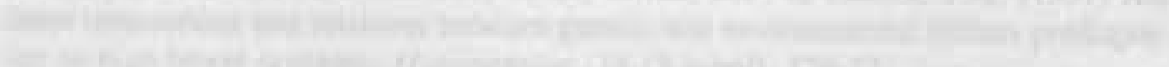

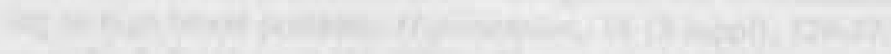

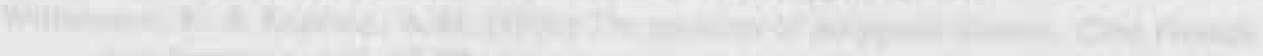

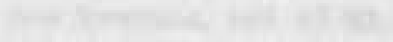

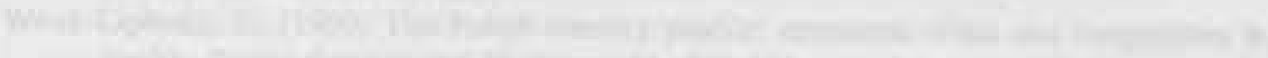
hin

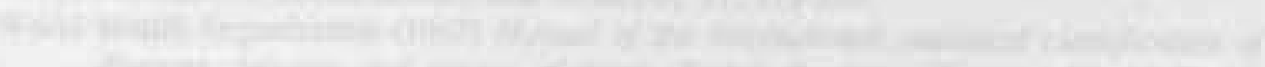
ran 


\section{Appendix I}

\section{KAUNAS DIAGNOSTIC CRITERIA}

Men with typical ECG or equivocal ECG with elevated enzymes and typical or atypical history were considered to have had a definite MI; men with typical history and elevated enzymes were also considered to have had a definite MI. Those men with a typical history, whose ECG and enzyme results did not place them in category "definite MI" and in whom there was no good evidence for another diagnosis were categorized as having had a probable or possible MI. Men with equivocal ECG changes without a typical history were not considered a MI case, nor were men with elevated enzymes or chest pain that could be explained by another cause. Probable and possible MIs could not be distinguished from definite MIs in the data set. 


\section{1 xibnsigut}

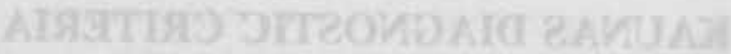

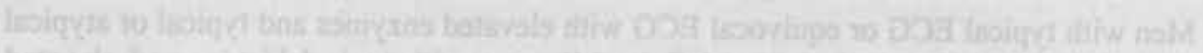

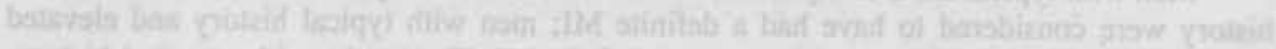

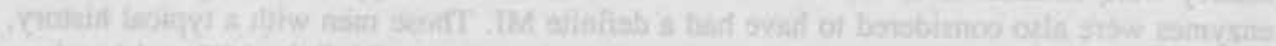

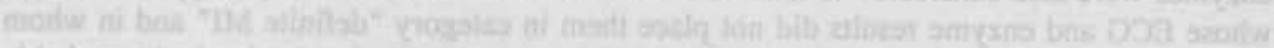

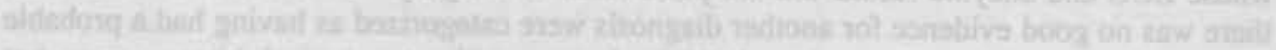

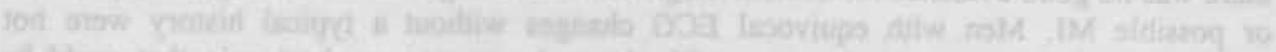

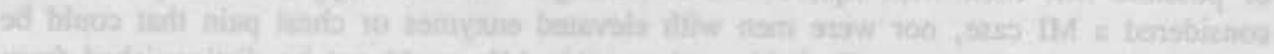

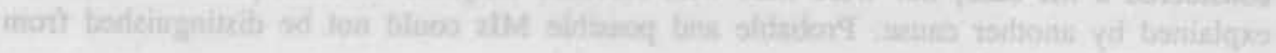

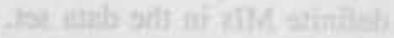




\section{ROTTERDAM MORBIDITY QUESTIONNAIRE}

\section{Questionnaire about heart complaints.}

On the next pages there are a number of questions about heart complaints. Perhaps in the previous 10 years (after 1972) you have or have not experienced heart complaints. Will you please fill in the following questions about heart complaints? For each question there are a number of answers. Read each answer carefully and mark the answer which is best suited in your case. First an example is given:

Have you experienced chest pain in the previous 10 years (after 1972)?
( ) yes
() no
() I am not sure

If you have experienced chest pain, then mark "yes". Like this:
(X) yes
() no
() I am not sure

If you have experienced no chest pain, then mark "no". Like this:
( ) yes
(X) no
( ) I am not sure

If you are not sure, then mark "I am not sure". Like this:
( ) yes
( ) no
(X) I am not sure

You may mark only one answer.

If your name or address is wrong, please fill in your correct name or address.

$$
\text { Name }
$$

Address

(1) Have you experienced severe heart complaints in the previous 10 years (after 1972)?
( ) yes
() no

(2) Has a physician ever told you that you have had a myocardial infarction in the previous 10 years (after 1972)?
() yes
( ) he told me that he did not know precisely
( ) it was about my heart, but I do not know what it was
( ) no 
(3) By whom have you been treated for your heart in the previous 10 years (after 1972)?

( ) I have not been treated for my heart

( ) cardiologist

() general practitioner

() general practitioner and cardiologist

(4) What is the name and the address of your general practitioner?

Name

Address

Place

(5) If you have been treated for your heart by a cardiologist in the previous 10 years (after 1972), what is his name?

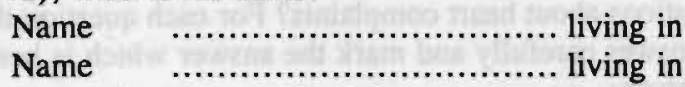

(6) Have you been hospitalized for your heart in the previous 10 years (after 1972)?
( ) yes
() no

If so, in which hospital and about when?

First hospitalization

Hospital
Month

Second hospitalization

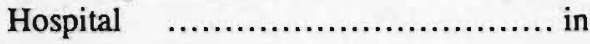

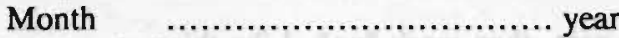

Third hospitalization

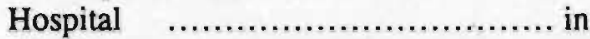

Month $\quad$.............................. year

Fourth hospitalization

Hospital ............................ in

Month $\quad . . . \ldots \ldots \ldots \ldots \ldots \ldots \ldots \ldots$ year

(7) Have you undergone a heart surgery in the previous 10 years (after 1972)?

( ) yes

() no

If so, when?

Hospital? (............................ in

(8) Have you undergone a heart catheterization in the previous 10 years (after 1972)?

() yes

() no

() I do not know what that is

If so, when?

Hospital?

in

(9) Have you been hospitalized for a stroke or a cerebral hemorrhage in the previous 10 years (after 1972)?

() yes

() no

If so, when?

Hospital? in 
(10) At present, do you smoke cigarettes?
() yes
() no

If so, how many cigarettes do you usually smoke per day?

........cigarettes per day.

(11) Do you smoke a pipe or cigars?
() only pipe
() only cigar
() both pipe and cigar
( ) neither pipe nor cigar

(12) Do you ever use medicine for chest pain?
() yes
() no
( ) I use medicine, but I do not know if it is for chest pain.

What is the name of the medicine?

(13) Do you use an anti-clotting medicine (sintrom, marcoumar, sintrommitis)?
() yes
() no

(14) Do you give us permission to ask further medical information from your general practitioner, if necessary?
() yes
() no
( ) yes, on the condition that.

(15) Do you give us permission to ask further medical information from your cardiologist, if necessary?
() yes
() no
( ) yes, on the condition that

(16) Do you have any further comments?

Date

signature

Thank you very much for your cooperation.

${ }^{1}$ In order to study the impact of individual advice given to KRIS participants, two questions about smoking habits (10 and 11) were added. 


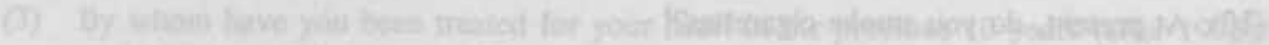

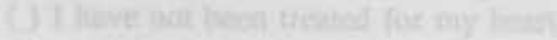

294 (3)

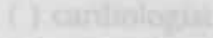

$\cos (a)$

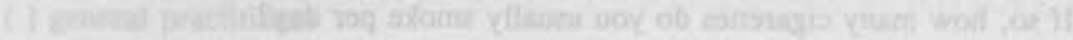

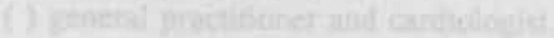

vath nact cattolentio

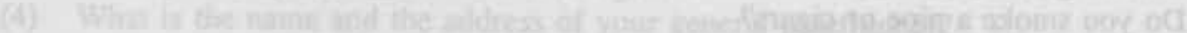

Whance

Nuthitias

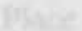

sclin vinon (t)

Tucita zina (

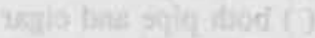

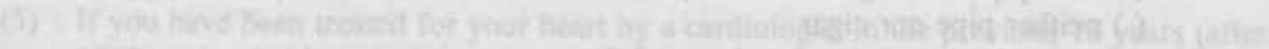

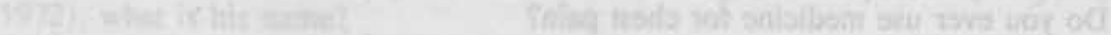

Bhase

betrate $\operatorname{man}(1)$ $\cos \left(\frac{10}{2}\right)$

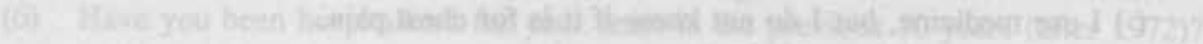
$(1, y=$

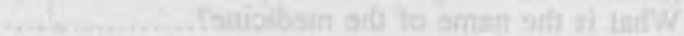

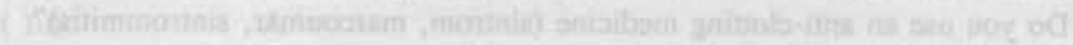

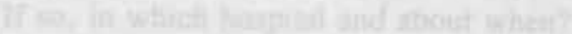

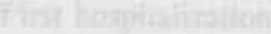

$\cos (0)$

$\operatorname{ant}(1)$

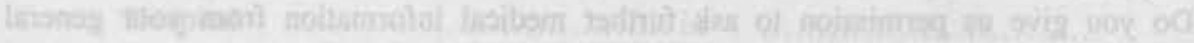

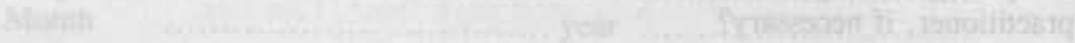

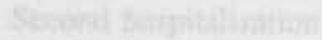

20in

Bitanitit

int

ont in

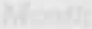

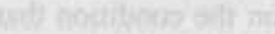

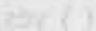

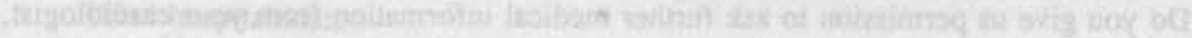

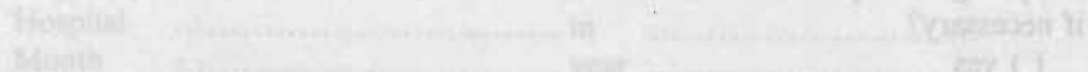

$\sin (t)$

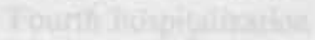

Ithe

ent (

Minina

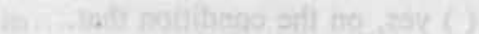

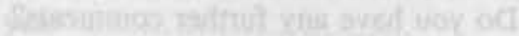

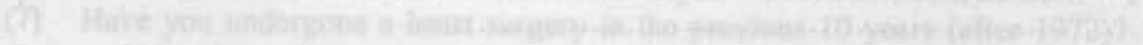

$(17 \times 0$

(1)

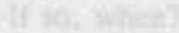

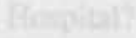

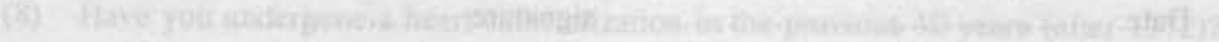

(4) 1 vat

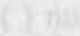

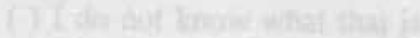

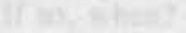

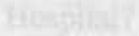

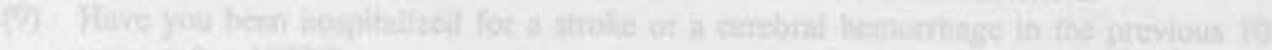

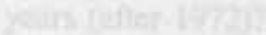

(7) $y=$

(bi) nate

fir sag whaten

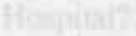

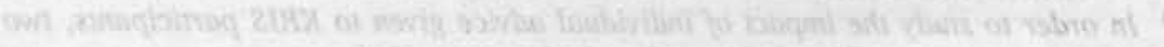

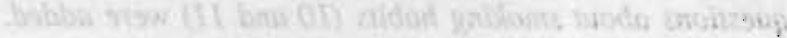




\section{Appendix III}

\section{ROTTERDAM DIAGNOSTIC CRITERIA}

Anamnesis, ECG, and enzyme-level data were used to classify cases based on the number of points accumulated. This coding scheme was developed in consultation with Prof. Dr. J. Pool.

\section{MI}

\section{Criteria}

\section{Anamnesis data}

Chest pain and 2 of the characteristics mentioned below:

2 points

Chest pain and 1 of the characteristics mentioned below:

1 point

Characteristics:

sudden start and increase

no relation to physical effort

duration longer than 30 minutes

pressing or spastic character

radiation

no reaction to nitroglycerine

None of these characteristics:

0 points

\section{ECGs}

Presence of the "injury current" and/or Minnesota codes 1.1, or 1.2 (Q-wave deviations), and/or 7.1 (left bundle block; in case of first MI), and/or the description of the ECG by cardiologist as "typical MI": 2 points Minnesota code 1.3 (moderate Q-wave deviations) and/or 5.1 or 5.2 (negative T)

and/or description of the ECG by cardiologist as "MI suspected": 1 point None of these:

0 points

\section{Enzymes}

SGOT and CPK values were both above a value considered normal in the specific hospital:

2 points

Either the SGOT value or the CPK value was above normal and/or the course of the enzyme values made the diagnosis of MI probable:

1 point

None of these:

0 points 


\section{Coding}

(1) When anamnesis, ECG, and enzyme-level data were available, the following coding was used:
0 - 1 points
no MI
2 - 3 points
possible MI
4 points probable MI
5 - 6 points definite MI

(2) When only two criteria were available for a case, a deviating coding scheme was used:

0 - 1 points no $\mathrm{MI}$

2 points possible MI

3 - 4 points probable MI

(3) When only a general clinical diagnosis was available for a case, the code "probable $\mathrm{MI}^{\text {" was assigned. }}$

\section{Angina Pectoris}

Anamnesis reported by a cardiologist indicative of angina pectoris and occlusions found during catheteriza- tion:
definite angina pectoris

Anamnesis reported by a cardiologist indicative of angina pectoris and deviations shown by ECG during pain or during physical exercise testing: Anamnesis reported by a cardiologist indicative of angina pectoris:

probable angina pectoris possible angina pectoris

\section{Cerebrovascular Attack}

The coding was based on information from general practitioners/cardiologists. When they had diagnosed cerebrovascular attack or transient ischemic attack, the coding was: C.VA: yes. 


\section{MEASUREMENT OF BIOLOGICAL RISK FACTORS ${ }^{1}$}

\section{ECGs}

Only resting ECGs were made. A one-channel ECG was used in Kaunas, and a Hewlett-Packard automatic and manual electocardiograph in Rotterdam. The speed of the tape was $50 \mathrm{~mm} / \mathrm{sec}$. Twelve standard leads were used: I, II, III, aVR, aVL, aVF, V1, V2, V3, V4, V5 and V6. The electrodes were placed in the standard positions after applying electrode cream (Mingograf in Kaunas, Redux in Rotterdam); Kaunas recorded five complexes per lead, Rotterdam at least three.

The observers were specially trained with the use of World Health Organization standard package of ECGs. In Kaunas all ECG tracings were coded by two independent observers; the results were compared afterwards, and in cases of disagreement a third observer was requested to make a decision. In Rotterdam quality control was carried out via external mechanisms: periodically a sample of coded ECGs was sent to a supervisor, and an annual refresher course was obligatory. The Minnesota Code (Rose \& Blackburn, 1968) was used to code the ECGs.

\section{Angina Pectoris}

The standard questionnaire published by Rose and Blackburn (1968) was used to assess angina pectoris.

\section{Blood Pressure}

Similar instruments for measuring blood pressure were used at the two centers. In addition, those responsible for measuring the blood pressure in Rotterdam had taken a course on general methodological and epidemiological principles and a course on the measurement of blood pressure. The latter was taught with an "octopus" stethoscope and a tape recording of the blood pressure sounds for the various Korotkov phases (obtained through the courtesy of Prof. G. Rose). The blood pressure was determined with a random zero sphygmomanometer (trademark Hawksley) and a Littman stethoscope. The random zero sphygmomanometer is a manometer designed especially for epidemiological purposes.

Before every measurement the baseline mercury level was changed at random; the cuff, after inflation to at least $240 \mathrm{mmHg}$, deflated at a constant rate of approximately $2 \mathrm{~mm} / \mathrm{sec}$. As the mercury column dropped, the systolic blood pressure and diastolic blood pressure were read as follows: (1) systolic blood pressure: level of the mercury at occurrence of tones that continued rhythmically (2) diastolic I: level of the mercury at sudden change of tones (i.e., the disappearance of the high component of tones, the so-called muffling) (3) diastolic II: level of the mercury at the disappearance of tones.

1 Source: Glasunov et al. (1981) 
The zero value was marked off in even millimeters, and the pressure was read to the nearest even millimeter; the true measurement was obtained by subtracting the baseline value (or zero value) from the observed value. This procedure was carried out twice, without removing the cuff. The two readings of the systolic blood pressure as well as the diastolic II blood pressure were averaged for data analysis.

\section{Plasma Glucose Tolerance and Cholesterol}

For the screening examination, a time-consuming glucose tolerance test after adequate fasting was not considered feasible. For this reason the test carried out during the screening examination was as follows: the participants were urged not to eat and drink during the two hours preceding the examination. At the beginning of the test the subject drank a solution of $75 \mathrm{~g}$ of glucose flavored with $0.5 \%$ citric acid in $200 \mathrm{ml}$ water. One hour later a venous blood sample was taken without stasis from one arm while the participant sat upright. Participants with a history of diabetes were excluded from the glucose tolerance test.

The blood sample was taken by means of a Vacutainer (Beckton \& Dickinson, type $3200 \mathrm{PS}$ ) containing $20 \mathrm{mg}$ of potassium oxalate and $25 \mathrm{mg}$ of sodium fluoride for every 10 $\mathrm{ml}$ of blood. The contents were mixed by inverting at least ten times and centrifuged within one hour at $1000 \times \mathrm{g}$ for 10 minutes. The plasma was then pipetted into three small tubes (40 $\mathrm{x} 10.5 \mathrm{~mm}$ ) which were closed and labelled. Two tubes were used for estimation of plasma cholesterol and plasma glucose; the third was frozen (-20 degrees Celsius) and stored for quality control purposes. Plasma glucose was measured by the o-toluidine reaction of Hultman (1959) and Cooper \& MacDaniel (1970).

Total plasma cholesterol was measured according to the modified 'direct' manual method of Huang et al. (1961) as described in detail in the Dutch Standardized Method NEN 2415 of The Netherlands Normalization Institute and The Netherlands Association for Clinical Chemistry.

Both analyses were checked continuously by the Lipid Reference Laboratory of the Center for Disease Control, Atlanta, USA against C.D.C. reference methods, and the results of laboratories in the two cities were compared at frequent intervals (Boerma et al., 1978).

\section{Body Mass Index}

The body mass index is a measure of an individual's weight, relative to his or her height. It is computed by $\mathrm{kg} / \mathrm{m}^{2}$. Height was measured during screening by means of a fixed vertical scale with a crossbar that was placed on top of the head, while the subject stood erect, without shoes, with the heels together, and with the eyes directed straight ahead. The measurement was read to the nearest $\mathrm{cm}$. Weight was measured by means of a level balance (Kaunas $0.05 \mathrm{~kg}$ scale, Rotterdam $0.1 \mathrm{~kg}$ scale) while the subject was dressed, but without jacket and shoes, with empty pockets. 


\section{Appendix V}

\section{MEASUREMENT OF LIFESTYLE RISK FACTORS ${ }^{1}$}

\section{Smoking}

In order to assess smoking habits, the questionnaire published by Rose \& Blackburn (1968) was submitted to the participants during the screening examination.

\section{Alcohol Consumption}

Alcohol consumption was assessed during screening examinations by means of the following questionnaire:

(1) Do you ever drink alcoholic beverages? (no, never; not now, did previously (one year or more ago); yes, occasionally; yes, regularly)

(2) How often? (once a year; once a month; once a week; several times a week, but not daily; daily)

(3) What do you normally drink? (beer only; wine only; spirits only; beer/wine; beer/spirits; wine/spirits; beer/wine/spirits)

(4) How much do you usually drink:

on one occasion

no beer

beer up to 1 ltr

more than 1 ltr

no wine

wine up to $0.5 \mathrm{ltr}$

$>0.5 \mathrm{ltr}$ but less than $1 \mathrm{ltr}$

more than $1 \mathrm{ltr}$

no spirit

spirits $^{\mathrm{b}}$ up to $100 \mathrm{cc}$

more than $100 \mathrm{cc}$

$(1 \mathrm{drink}=40 \mathrm{cc})$ daily

no beer

up to $1 \mathrm{ltr}$

more than 1 ltr

no wine

up to $0.5 \mathrm{ltr}$

$>0.5$ ltr but less than 1

more than 1 ltr

no spirit

up to $100 \mathrm{cc}$

more than $100 \mathrm{cc}$

(1 drink $=40 \mathrm{cc})$

red and white wine, sherry, vermouth, etc.

whiskey, jenever, vodka, cognac, liquor

' Source: Glasunov et al. (1981) 


\section{Physical Activity}

During screening, physical activity was assessed for work, means of transportation to work, and activities in leisure time respectively. The questions were as follows:

(1) How many hours do you spend at work sitting?

(2) How many hours do you spend at work standing?

(3) How many hours do you spend at work moving about?

(4) How many hours do you spend at work carrying a load?

(5) Distance home-work: minutes a day bicycling?

(6) Distance home-work: minutes a day walking?

(7) Distance home-work: minutes a day by public transport?

(8) Distance home-work: minutes a day by car, motorcycle etc.?

(9) How many leisure hours a week do you spend walking, in wintertime?

(10) How many leisure hours a week do you spend walking, in summertime?

(11) How many leisure hours a week are you moderately or very active, in wintertime?

(12) How many leisure hours a week are you moderately or very active, in summertime?

Table I shows the metabolic ratios used to compute the total physical activity score (Reiff et al., 1964). For example, someone who is engaged in standing 6 hours a day, carrying objects 2 hours a day, sitting on his bicycle for 40 minutes a day, spending 3 hours a week moderately or very active the whole year received the following score: $(6 * 3 * 5)+$ $(2 * 5 * 5)+(40 * 4 *(5 / 60))+(2 *(3 * 5 * 0.5))=168.3$

Table I Metabolic ratios and factors for converting to hours per week.

\section{Metabolic Ratio}

Oecupation

Transport

Sitting

Standing

Moving

Carrying

Leisure
Bicycle
Walking

Walking winter

Walking summer

Moderately/very active winter

Moderately/very active summer
2

3

4

5

\section{4}

4

4

4

5

5
Unit

Conversion factor

$\begin{array}{ll}\text { h/day } & 5 \\ \text { h/day } & 5 \\ \text { h/day } & 5 \\ \text { h/day } & 5\end{array}$

$\begin{array}{ll}\mathrm{min} / \text { day } & 5 / 60 \\ \mathrm{~min} / \text { day } & 5 / 60 \\ & \\ \mathrm{~h} / \text { week } & 1 / 2 \\ \mathrm{~h} / \text { week } & 1 / 2 \\ \mathrm{~h} / \text { week } & 1 / 2 \\ \text { h/week } & 1 / 2\end{array}$




\section{MEASUREMENT OF TYPE A BEHAVIOR PATTERN}

To assess this coronary-prone behavior pattern, the JAS, Form B, was used during the screening examinations (Jenkins et al., 1979). This questionnaire consists of 61 items (Glasunov et al., 1981). The Short Form N of the JAS is based on this questionnaire and consists of the following 13 questions:

(1) When you are under pressure or having trouble, do you:

a. take measures to solve the problem immediately?

b. plan carefully before you start doing anything?

(2) When you are listening to someone who talks a long time before coming to the point, do you feel like hurrying him up?
a. frequently
b. occasionally
c. almost never

(3) How often do you actually "put words into a person's mouth" to speed up the conversation?
a. often
b. occasionally
c. almost never

(4) If you tell your wife or a friend that you will come at a certain time, how often are you late?
a. sometimes
b. almost never
c. never
d. I am absolutely never late.

(5) When you were young, did people think:

a. that you were often overexerting yourself and eagerly wanted to be the first and the best in everything?

b. that you were sometimes inclined to overexert yourself and liked to be the first and the best?

c. that you were usually relaxed and took things as they came?

d. that you were always relaxed and were able to take things very easily?

(6) What would your wife's opinion (or your best friend's opinion) of you be?

a. I often overexert myself and eagerly want to be the first and the best in everything.

b. I am sometimes inclined to overexert myself and like to be the first and the best.

c. I am usually relaxed and take things as they come.

d. I am always relaxed and I am able to take things very easily.

(7) How would your wife or your best friend rate your level of activity generally?
a. too slow; should be more active
b. about average; is busy much of the time
c. too active; needs to slow down 
(8) What was your temper like when you were younger?
a. hot-tempered and hardly controlled
b. strong but controllable
c. no problem
d. I hardly ever got angry

(9) Would people who know you well agree that you have less energy than most people?
a. definitely yes
b. probably yes
c. probably no
d. definitely no

(10) When you are one of a group, do the others expect you to take charge?
a. seldom
b. as often they expect others to do so
c. more often than they expect others to do so

(11) Compared to others who do the same work, do you have to be accurate and pay attention to details:
a. much more
b. a little more
c. the same
d. a little less
e. much less

(12) Compared to others who do the same work, do you approach life in general:
a. much more seriously
b. a little more seriously
c. the same
d. a little less seriously
e. much less seriously

(13) When you were in high school or college, were you a member of one or more athletic teams?
a. no
b. yes, one team
c. yes, two or more teams

Answers to these questions were differently weighted using the weights in Table II below. Since item 13 was missing in the KRIS, all participants were assigned the score 1.30 for that item. Men who did not answer more than 3 items were not included in the scale. The following calculations made the scale comparable with the standardizing population for the Western Collaborative Group Studies. Weights were added for each individual. Subsequently, 22.54 was subtracted and the result divided by 0.62 . Higher values on this score indicate more type A behavior. 
Table II Weights given to responses to 13 questions of the JAS.

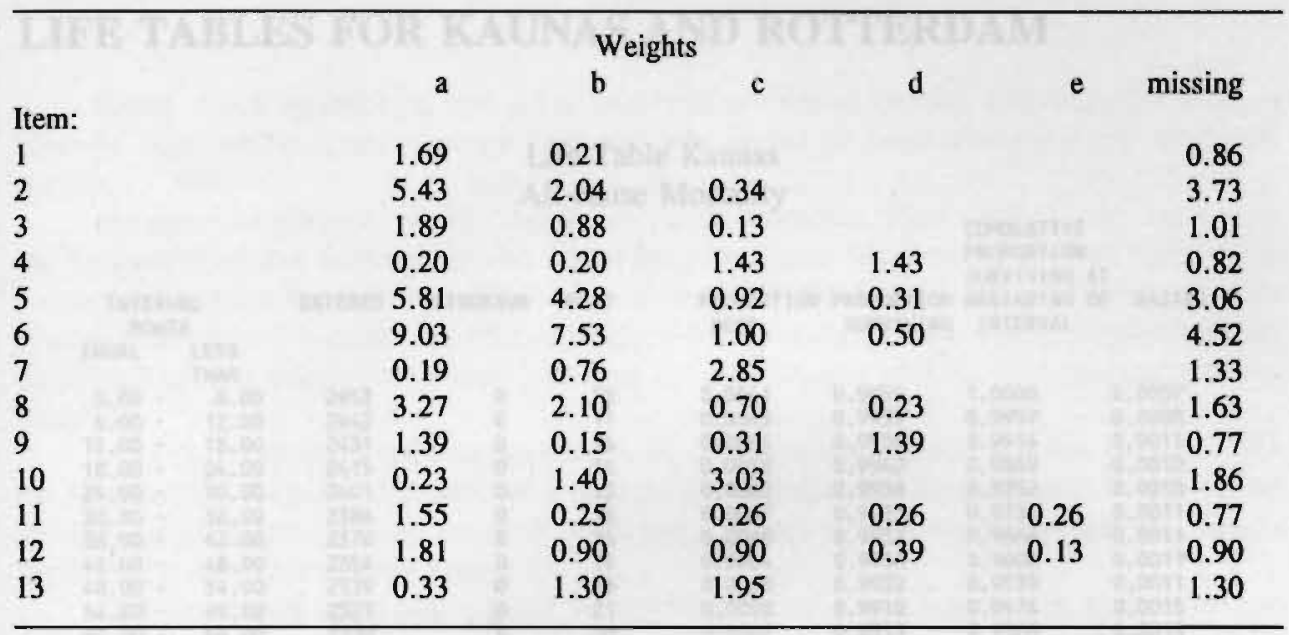




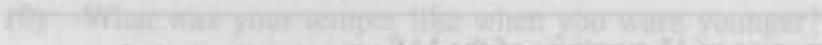

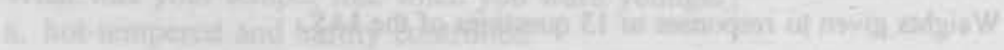

Ti=0

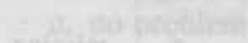

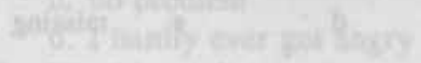

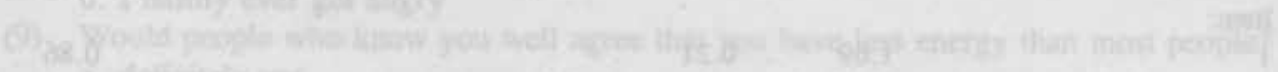

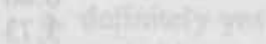

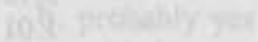

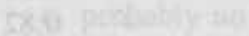

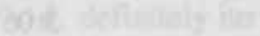

Fay

IED

HCE

Fito

k41:

(ixy 17

(10.7?

(12)

199.9

$32 x^{2}+2$

(10:4)

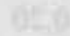

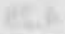

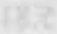

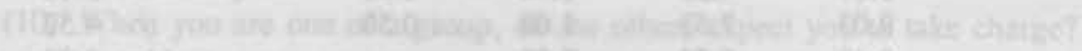

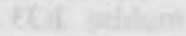

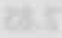
in:
$\operatorname{tin}$

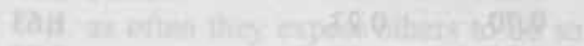

(4) 5

Fat 1

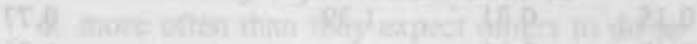

at.

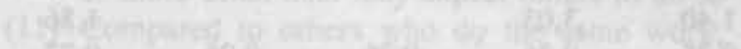

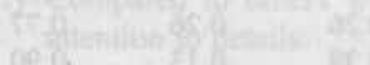

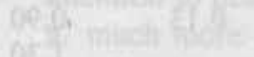

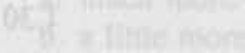
$(100)$
00,0
201

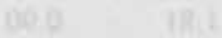

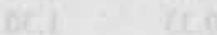

(14:57)
$14: 12$

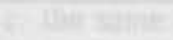

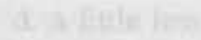

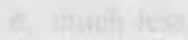

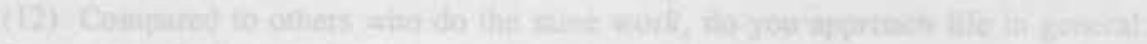

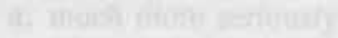

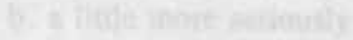

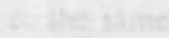

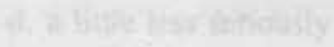

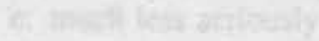

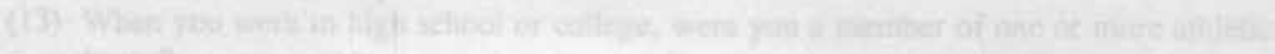

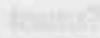

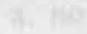

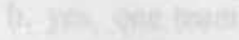

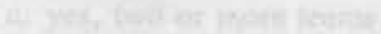

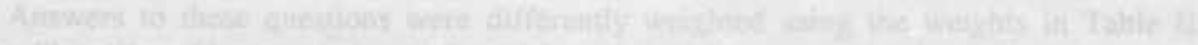

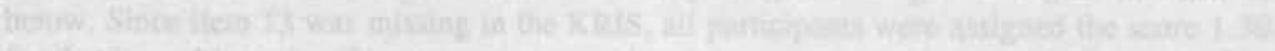

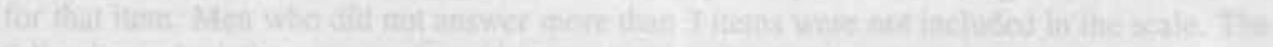

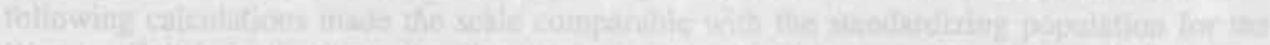

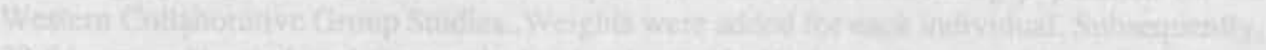

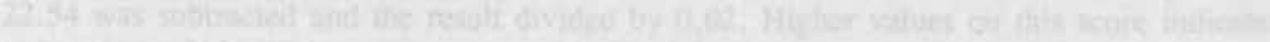

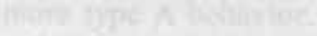




\section{LIFE TABLES FOR KAUNAS AND ROTTERDAM}

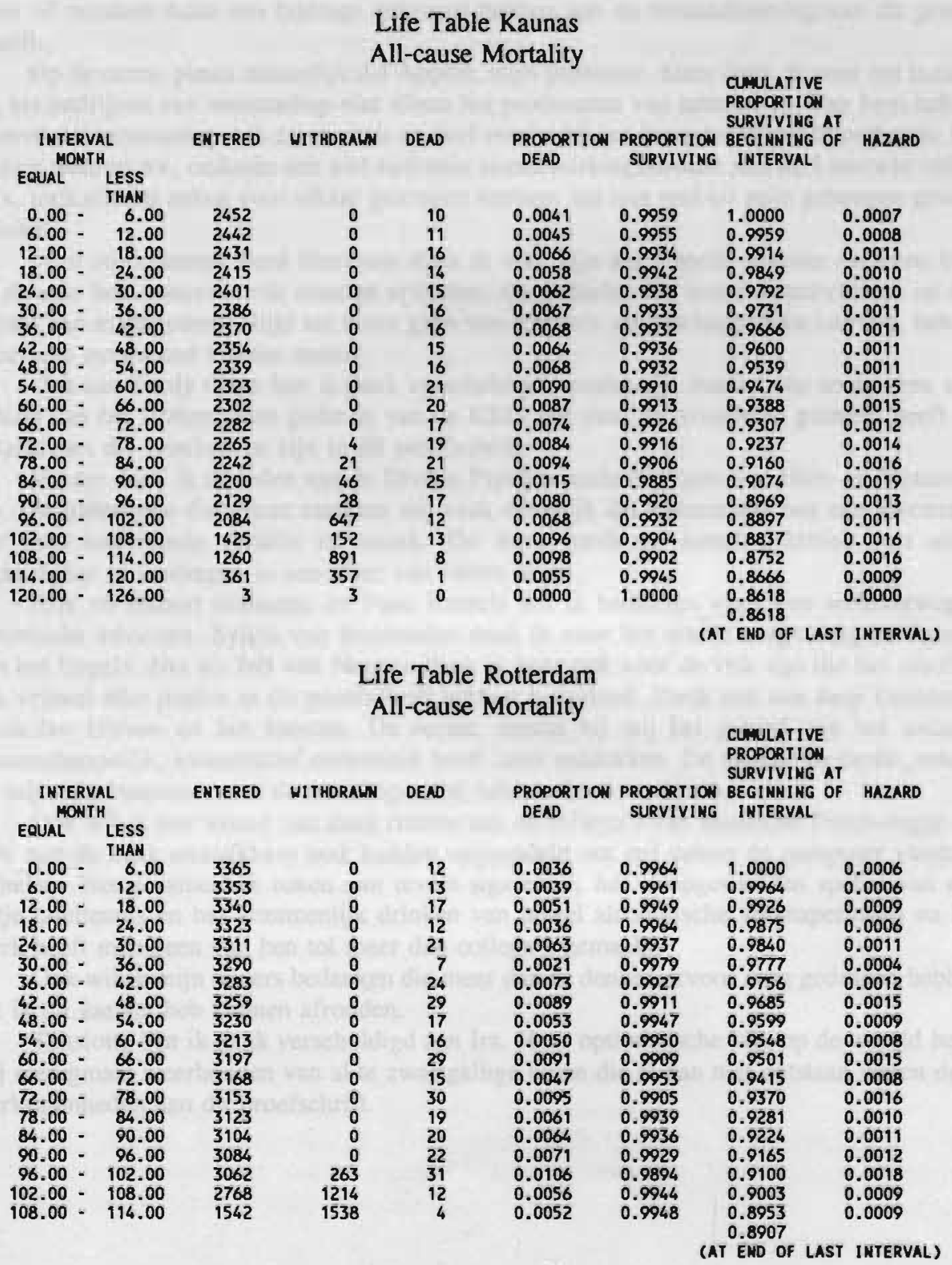




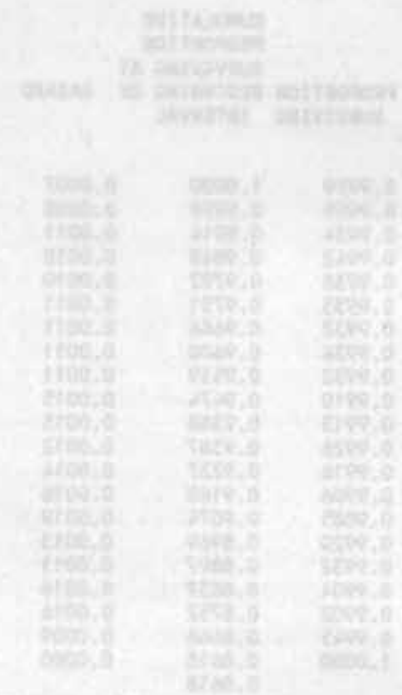

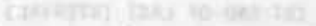

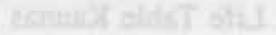 \\ vilingold waratith}

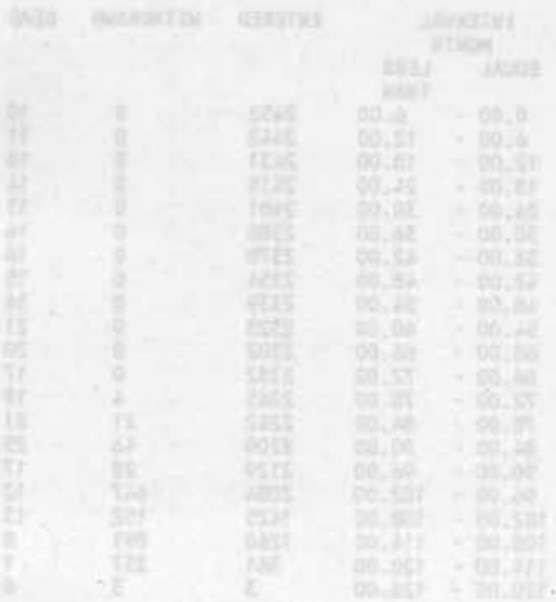

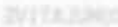

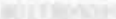

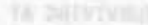

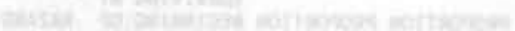

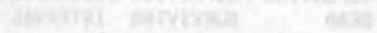

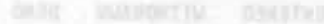

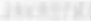

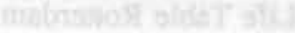

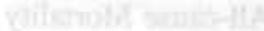

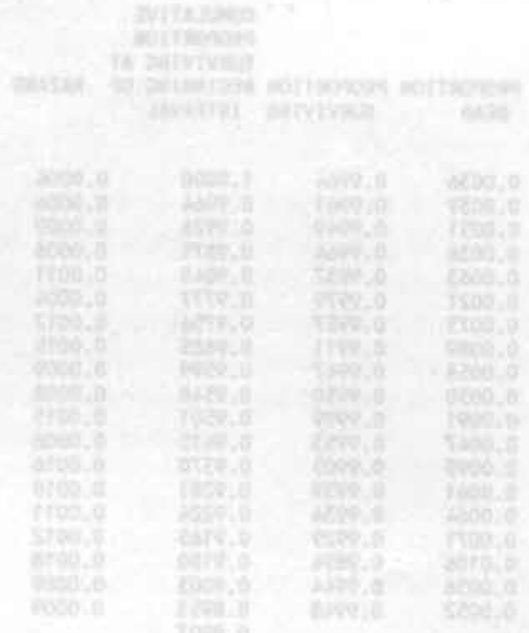

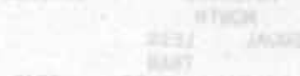

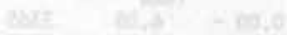

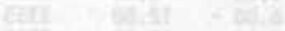

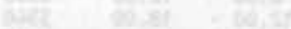

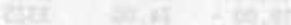

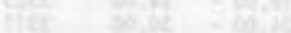

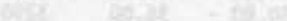

arata

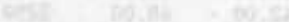

(4)

cirt-

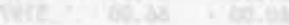

towe

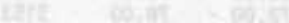

변.

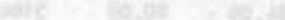

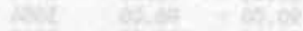

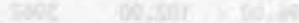

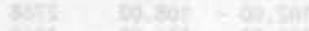
702014.

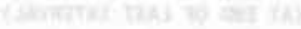


Graag wil ik op het eind van dit proefschrift een aantal mensen bedanken die allen in meer of mindere mate een bijdrage geleverd hebben aan de totstandkoming van dit proefschrift.

Op de eerste plaats natuurlijk Ad Appels, mijn promotor. Hem dank ik voor het inzicht dat het bedrijven van wetenschap niet alleen het produceren van tabellen is. Van hem heb ik geleerd dat wetenschap ook creativiteit en durf vereist bij het formuleren van hypothesen. De manier waarop we, ondanks een niet optimale samenwerkingssituatie met de Litouwse collega's, toch allerlei zaken voor elkaar gekregen hebben, zal niet snel uit mijn geheugen gewist worden.

Mijn co-promotor Ferd Sturmans dank ik voor zijn altijd snelle reacties en frisse blik op de vele hem toegestuurde concept artikelen. De gedachte dat iemands activiteiten op het gebied van management altijd ten koste gaan van iemands wetenschappelijke inbreng, heb ik door hem overboord moeten zetten.

Ook aan Ferdy Otten ben ik dank verschuldigd, omdat zijn manier van analyseren met behulp van het Rotterdamse gedeelte van de KRIS mij vaak tot voorbeeld gestrekt heeft bij de analyses die beschreven zijn in dit proefschrift.

Verder dank ik de leden van de Divisie Psychosociale Factoren van Hart- en Vaatziekten. De onderlinge discussies maakten mij vaak duidelijk dat wetenschap ook een kwestie is van inter-individuele variatie in smaak. De soms harde en terechte kritiek was altijd makkelijker te verdragen in een sfeer van 'entre nous'.

Erik en Hubert Schouten en Fons Kessels wil ik bedanken voor hun weloverwogen statistische adviezen. Sylvia van Roosmalen dank ik voor het uiterst zorgvuldig corrigeren van het Engels. Net als Isel van Noppen dank ik haar ook voor de vele tips die het uiterlijk van vrijwel elke pagina in dit proefschrift hebben beïnvloed. Dank ook aan Jaap Dronkers, Henk-Jan Dirven en Jan Joosten. De eerste, omdat hij mij het gebied van het sociaalwetenschappelijk, kwantitatief onderzoek heeft laten ontdekken. De tweede en derde, omdat zij mij mijn interesse voor de sociologie niet hebben laten verliezen.

Ook wil ik een woord van dank richten aan de collega's van Medische Psychologie die zich met de vaak ondankbare taak hadden opgezadeld om mij achter de computer vandaan te halen. Het gezamenlijk roken van teveel sigaretten, het te opgewonden spelen van een potje tafeltennis en het gezamenlijk drinken van teveel alcoholische versnaperingen na het werk heeft menigeen van hen tot meer dan collega's gemaakt.

Ook wil ik mijn ouders bedanken die meer dan ze denken ervoor zorg gedragen hebben dat ik dit karwei heb kunnen afronden.

Tenslotte ben ik dank verschuldigd aan Ira. Haar optimistische kijk op de wereld heeft mij menigmaal weerhouden van al te zwartgallige buien die al dan niet ontstaan waren door werkzaamheden aan dit proefschrift. 


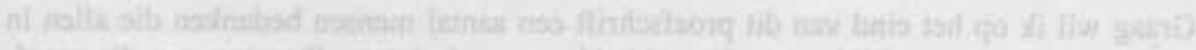

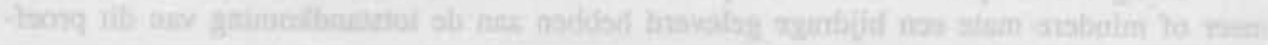

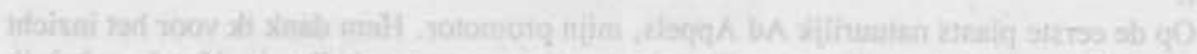
(4)

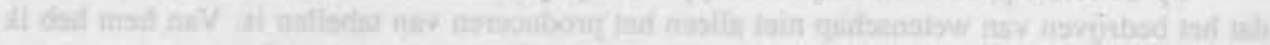

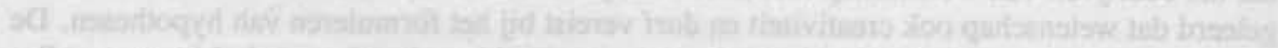

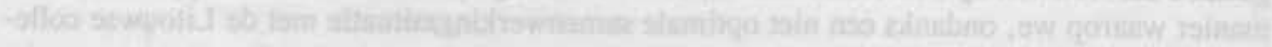

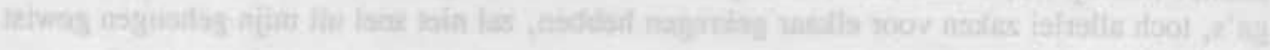

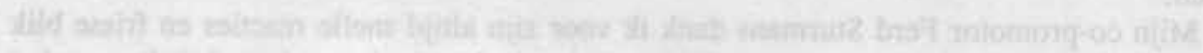

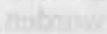

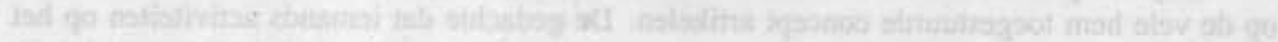

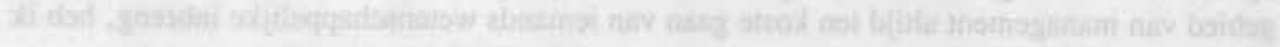

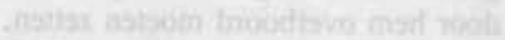

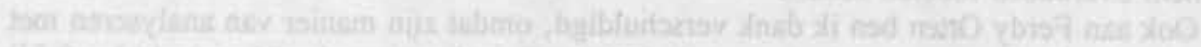

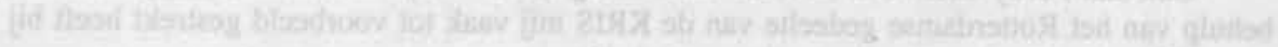

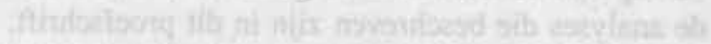

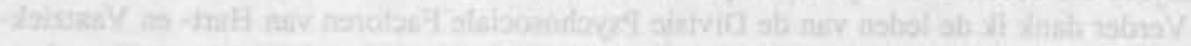

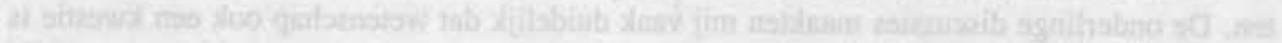

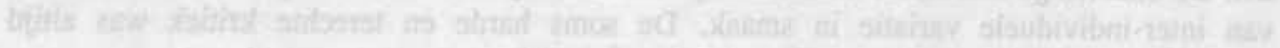

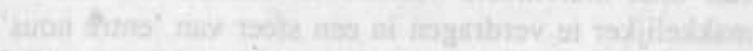

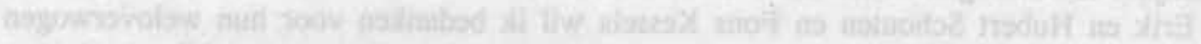

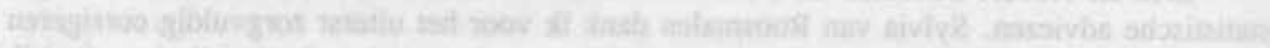

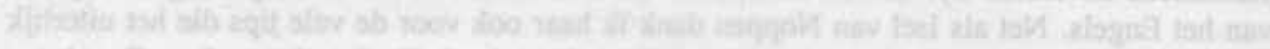

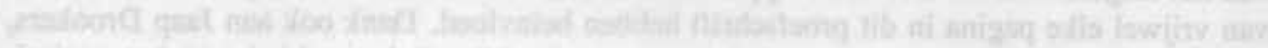

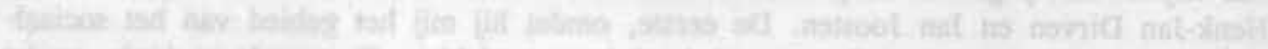

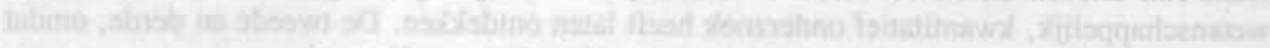

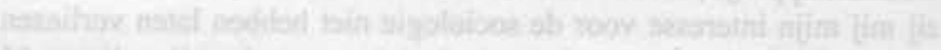

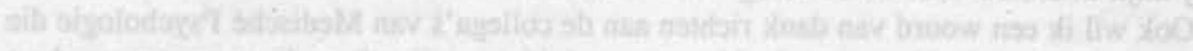

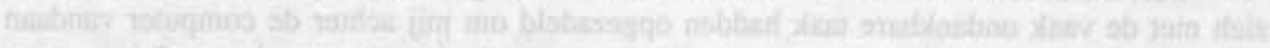

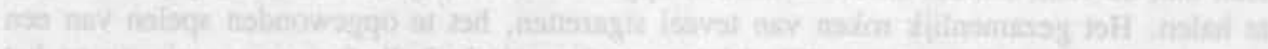

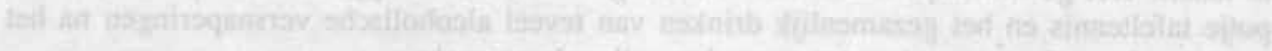

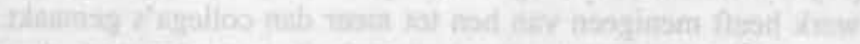

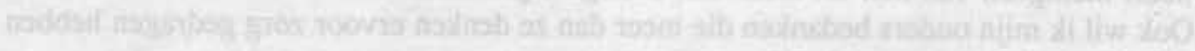

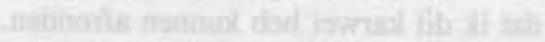

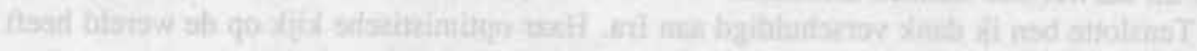

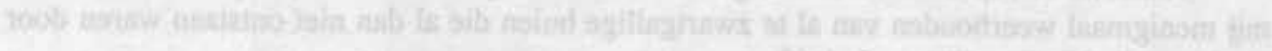

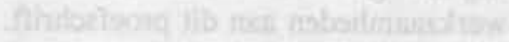


Appels, A., Bosma, H., Sturmans, F., Schuurman, J. \& Mulder, P. (1993) KRIS follow-up XIII: Connubium en sterftekans. Tijdschrift voor Sociale Gezondheidszorg, 71, 87-90. Appels, A., Bosma, H., Grabauskas, V., Gostautas, A. \& Sturmans, F. (1994,) The followup of the Kaunas-Rotterdam Intervention Study (KRIS). Copenhagen: WHO. (in press). Appels, A., Bosma, H., Grabauskas, V., Gostautas, A. \& Sturmans, F. (1994b) Self-rated health and mortality. (submitted).

Bosma, H. (1987) De slag om de leerling. Instituut voor Sociaal-wetenschappelijk Onderzoek (IVA): Tilburg.

Bosma, H., Appels, A., Sturmans, F., Mulder, P. \& Schuurman, J. (1991,) KRIS follow-up $X$ : De invloed van de sociaal-economische status van de vrouw op de levensverwachting van de man. Verschillen in morbiditeit en mortaliteit gedurende een follow-up van circa 10 jaar. Tijdschrift voor Sociale Gezondheidszorg, 69, 350-356.

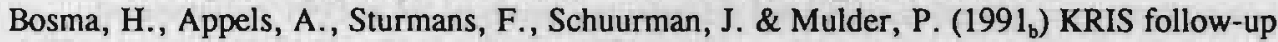
IX: Subjectief ervaren gezondheid en sterftekans. Verschillen in sterfte gedurende een follow-up van circa 10 jaar. Tijdschrift voor Sociale Gezondheidszorg, 69, 299-305.

Bosma, H., Dronkers, J. \& Hagenaars, J. (1991 ${ }_{c}$ ) The consequences of educational reform; Changes in educational attainment processes of pupils at secondary schools (Use of the loglinear method).

In: M.J. Peláez (Red.) Comparative sociology of family, health and education. Spain Zaragoza: Graficas Cometa.

Bosma, H., Appels, A., Sturmans, F. \& Grabauskas, V. (1992.) Marital status and life expectancy in Lithuania and the Netherlands. American Journal of Epidemiology, 136, 995 (abstract).

Bosma, H., Appels, A., Sturmans, F., Mulder, P. \& Schuurman, J. (1992 $)$ KRIS follow-up XI: De invloed van niet-somatische factoren op de levensverwachting bij personen met tekenen van een hartlijden. Verschillen in mortaliteit gedurende een follow-up van circa 10 jaar. Tijdschrift voor Sociale Gezondheidszorg, 70, 293-298.

Bosma, H., Appels, A., Grabauskas, V., Gostautas, A. \& Sturmans, F. (1994_) Marital status and mortality in Lithuania and the Netherlands. Results from the WHO KaunasRotterdam Intervention Study (KRIS). (submitted).

Bosma, H., Appels, A., Grabauskas, V., Gostautas, A. \& Sturmans, F. (1994b) The changing gradient of the association between socioeconomic status and risk of coronary heart disease in Lithuania and the Netherlands. Results from the WHO KaunasRotterdam Intervention Study (KRIS). (submitted).

Bosma, H., Appels, A., Sturmans, F., Grabauskas, V. \& Gostautas, A. (1994 ${ }_{c}$ ) Socioeconomic status and mortality in Lithuania and the Netherlands. Results from the WHO Kaunas-Rotterdam Intervention Study (KRIS). (submitted).

Bosma, H., Appels, A., Sturmans, F., Grabauskas, V. \& Gostautas, A. (1994 $)$ Educational level of spouses and risk of mortality. Results from the WHO Kaunas-Rotterdam Intervention Study (KRIS). (submitted). 
Bosma, H., Appels, A., Sturmans, F., Grabauskas, V. \& Gostautas, A. (1994) Differences in mortality and coronary heart disease between Lithuania and the Netherlands. Results from the WHO Kaunas-Rotterdam Intervention Study. International Journal of Epidemiology. (accepted).

Dronkers, J. \& Bosma, H. (1990) The consequences of educational reform for the educational opportunities of children of different social classes in secondary schools. Zeitschrifi für Socialisationsforschung und Erziehungssociologie, 10, 268-278.

Gostautas, A., Appels, A. \& Bosma, H. (1992) Socialiniv reiksniv itaka gyrentojv poziurivi i sveitkata.

In: S. Rinkinys (Red.) Socialniai sveikatos aspektai. Kaunas: Lietuvos Mokslininkv Sajunga. 


\section{CURRICULUM VITAE}

Hans Bosma werd in Limbricht geboren op 22 november 1963. In 1983 behaalde hij het gymnasium diploma aan het Bisschoppelijk College te Sittard. Aansluitend ging hij sociologie studeren aan de Katholieke Universiteit Brabant in Tilburg. In 1989 rondde hij de studie af in de afstudeerrichting Onderwijswetenschappen. De laatste twee jaar van zijn studie is hij, als student-assistent, verbonden geweest aan diverse onderzoeksprojecten van de vakgroep sociologie en het Instituut voor Sociaal-Wetenschappelijk Onderzoek (IVA) te Tilburg.

Van augustus 1989 tot september 1993 is hij als toegevoegd onderzoeker werkzaam geweest bij de vakgroep Medische Psychologie van de Rijksuniversiteit Limburg in Maastricht. Het onderzoek, waarvan dit proefschrift een verslag vormt, werd uitgevoerd binnen divisie VIII "Psychosociale factoren van hart- en vaatziekten" (leider: Prof.dr. A. Appels).

In november 1993 is hij in dienst getreden bij het Centraal Bureau voor de Statistiek te Heerlen, hoofdafdeling Statistieken van Onderwijs en Wetenschappen, afdeling Cohorten. 
